Supporting Information

\title{
Enantioselective Rh-Catalyzed Anti-Markovnikov Hydroformylation of 1,1-Disubstituted Allylic Alcohols and Amines: An Efficient Route to Chiral Lactones and Lactams
}

\author{
Cai You, ${ }^{a}$ Shuailong Li, ${ }^{a}$ Xiuxiu Li, ${ }^{a}$ Hui Lv ${ }^{a, c}$ Xumu Zhang ${ }^{b}$ \\ ${ }^{a}$ Key Laboratory of Biomedical Polymers of Ministry of Education \& College of Chemistry and \\ Molecular Sciences, Wuhan University, Wuhan, Hubei 430072, P. R. China \\ ${ }^{b}$ Grubbs Institute and Department of Chemistry, Southern University of Science and Technology, \\ Shenzhen, Guangdong 518000, P. R. China \\ ${ }^{c}$ Engineering Research Center of Organosilicon Compounds \& Materials, Ministry of Education, \\ Sauvage Center for Molecular Sciences, College of Chemistry and Molecular Sciences, Wuhan \\ University, Wuhan, Hubei, 430072, China.
}

Table of Contents

1. General Information $\quad$ S2

2. Procedures for the preparation of $(S, S)$-DTBM-YanPhos $\quad$ S2

3. General procedure for asymmetric hydroformylation $\quad$ S3

4. Procedures for gram-scale asymmetric hydroformylation of $\mathbf{1 p} \quad \mathrm{S} 12$

5. Synthesis of 3-(3, 5-dimethylphenyl)tetrahydrofuran 6a $\quad$ S13

6. Synthesis of (4S)-2-allyl-4-(3, 5-dimethylphenyl)tetrahydrofuran $\quad \mathbf{6 b} \quad \mathrm{S} 13$

7. Synthesis of $(S)-2-(3,5$-dimethylphenyl)butane-1,4-diol 6c $\quad S 14$

8. General procedure for the AHF of I with $(S, S)$-DTB-YanPhos $\quad \mathrm{S} 14$

9. References $\quad$ S15

10. NMR spectra $\quad$ S16

11. HPLC spectra $\quad$ S46 
1. General Information. All reactions and manipulations that were sensitive to moisture or air were performed in a nitrogen-filled glovebox or using standard Schlenk techniques, unless otherwise noted. Solvents were dried with standard procedures, degassed with $\mathrm{N}_{2}$ and transferred by syringe. NMR spectra were recorded on Bruker ADVANCE III (400 MHz) spectrometers for ${ }^{1} \mathrm{H}$ NMR and ${ }^{13} \mathrm{C}$ NMR. $\mathrm{CDCl}_{3}$ was the solvent used for the NMR analysis, with tetramethylsilane as the internal standard. Chemical shifts were reported upfield to TMS $(0.00 \mathrm{ppm})$ for ${ }^{1} \mathrm{H}$ NMR and relative to $\mathrm{CDCl}_{3}$ (77.3 ppm) for ${ }^{13} \mathrm{C}$ NMR. Optical rotation was determined using a Perkin Elmer 343 polarimeter. HPLC analysis was conducted on an Agilent 1260 Series instrument. Thin layer chromatography (TLC) was performed on EM reagents $0.25 \mathrm{~mm}$ silica 60 F plates. All new products were further characterized by HRMS. A positive ion mass spectrum of sample was acquired on a Thermo LTQ-FT mass spectrometer with an electrospray ionization source.

\section{Procedures for the preparation of $(S, S)$-DTBM-YanPhos}<smiles>CCCCc1ccc2ccccc2c1-c1c(NCc2ccccc2)ccc2ccccc12</smiles>

A

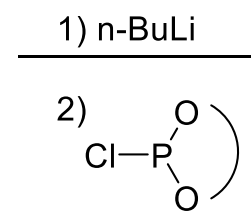

B<smiles>CCCc1ccc2ccccc2c1-c1c(N(Cc2ccccc2)C(C)(F)F)ccc2ccccc12</smiles>
$\mathrm{Bn} \mathrm{O}$

$(S, S)$-DTBM-YanPhos was prepared according to the literature. ${ }^{1,2}$ To a solution of A (1 $\mathrm{mmol})$ in THF $(10 \mathrm{~mL})$ at $-78{ }^{\circ} \mathrm{C}$ was added dropwise n-BuLi $(1.5 \mathrm{mmol}, 2.4 \mathrm{M}$ in hexane). The reaction mixture was stirred for $1 \mathrm{~h}$ to give a deep red solution, and $\mathbf{B}$ (1.5 mmol) in THF ( $5 \mathrm{~mL})$ was added dropwise. After addition, the cooling bath was removed and the mixture was stirred at room temperature overnight. The volatiles were evaporated under reduced pressure. To the residue was added $\mathrm{CH}_{2} \mathrm{Cl}_{2}(10 \mathrm{~mL})$, and the mixture was filtered to remove the salt. The filtration was concentrated and subjected to chromatography on silica gel (eluted with hexane/EtOAc 100:1 to 10:1) to afford pure ligands.

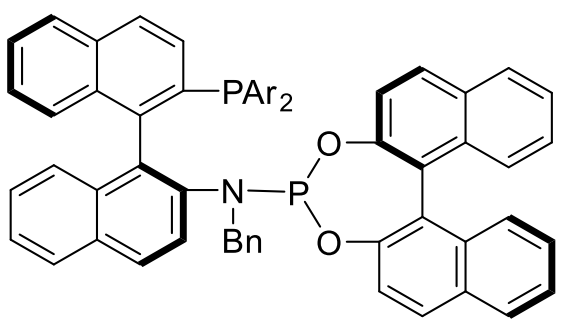

(S,S)-DTBM-YanPhos

$\mathrm{Ar}=3,5-\mathrm{t}-\mathrm{Bu}-4-\mathrm{OMe}-\mathrm{C}_{6} \mathrm{H}_{2}$

$(\boldsymbol{S}, \boldsymbol{S})$-DTBM-YanPhos: Light yellow solid, $513 \mathrm{mg}, 45 \%$ yield. $[\alpha]_{\mathrm{D}}{ }^{25}=+9.5(\mathrm{c}=1.0$, $\left.\mathrm{CHCl}_{3}\right) .{ }^{1} \mathrm{H} \mathrm{NMR}\left(400 \mathrm{MHz}, \mathrm{CDCl}_{3}\right) \delta 8.18(\mathrm{~d}, J=8.6 \mathrm{~Hz}, 1 \mathrm{H}), 7.98(\mathrm{~d}, J=8.2 \mathrm{~Hz}$, $1 \mathrm{H}), 7.86(\mathrm{t}, J=8.4 \mathrm{~Hz}, 2 \mathrm{H}), 7.75-7.64(\mathrm{~m}, 4 \mathrm{H}), 7.51(\mathrm{~d}, J=8.8 \mathrm{~Hz}, 1 \mathrm{H}), 7.44(\mathrm{~s}, 1 \mathrm{H})$, 
7.31-7.39 (m, 4H), 7.19-7.16 (m, 6H), $7.07(\mathrm{~d}, J=5.7 \mathrm{~Hz}, 2 \mathrm{H}), 6.98(\mathrm{~d}, J=6.9 \mathrm{~Hz}, 2 \mathrm{H})$, $6.87(\mathrm{~d}, J=8.4 \mathrm{~Hz}, 2 \mathrm{H}), 6.50-6.46(\mathrm{~m}, 1 \mathrm{H}), 6.40(\mathrm{~d}, J=8.4 \mathrm{~Hz}, 1 \mathrm{H}), 5.82(\mathrm{~d}, J=8.8$ $\mathrm{Hz}, 1 \mathrm{H}), 4.40$ (d, $J=14.4 \mathrm{~Hz}, 1 \mathrm{H}), 3.78$ (d, $J=14.2 \mathrm{~Hz}, 1 \mathrm{H}), 3.64(\mathrm{~s}, 3 \mathrm{H}), 3.62(\mathrm{~s}, 3 \mathrm{H})$, $2.35(\mathrm{~s}, 2 \mathrm{H}), 1.55(\mathrm{~s}, 2 \mathrm{H}), 1.22(\mathrm{~s}, 18 \mathrm{H}), 1.14(\mathrm{~s}, 18 \mathrm{H}) \mathrm{ppm} .{ }^{13} \mathrm{C} \mathrm{NMR}\left(101 \mathrm{MHz}, \mathrm{CDCl}_{3}\right)$ $\delta 160.60,159.54,150.09,149.60,145.47,143.63,143.58,143.40,143.32,138.48$, $137.76,134.07,133.80,133.57,133.01,132.14,131.75,131.57,130.70,130.65$, $130.28,129.88,129.32$, 128.51, 128.34, 127.99, 127.90, 127.74, 127.42, 127.29, $127.15,126.90,126.83,126.10,125.65,125.58,124.89,124.72,124.58,122.65$, $121.88,64.61,64.46,36.00,35.94,32.25,32.16 \mathrm{ppm} .{ }^{31} \mathrm{P}$ NMR $\left(162 \mathrm{MHz}, \mathrm{CDCl}_{3}\right) \delta$ $139.90(\mathrm{~d}, J=16.8 \mathrm{~Hz}),-15.55(\mathrm{~d}, J=16.7 \mathrm{~Hz})$. HRMS calculated $[\mathrm{M}+\mathrm{H}]^{+}$for $\mathrm{C}_{77} \mathrm{H}_{78} \mathrm{NO}_{4} \mathrm{P}_{2}=1142.5401$, found: 1142.5386 .

\section{General procedure for asymmetric hydroformylation and oxidation reaction}

In a glovebox filled with argon, to a $5 \mathrm{ml}$ vial equipped with a magnetic bar was added $(S, S)$-DTBM-YanPhos $(0.024 \mathrm{mmol})$ and $\mathrm{Rh}(\mathrm{acac})(\mathrm{CO})_{2}(0.008 \mathrm{mmol}$ in $1 \mathrm{~mL}$ toluene $)$. After stirring for $10 \mathrm{~min}$, the mixture was charged to substrate $(0.2 \mathrm{mmol})$. The vial was transferred into an autoclave and taken out of the glovebox. The argon gas was replacement with hydrogen gas for three times, and then hydrogen ( 2.5 bar) and carbon monoxide $(2.5 \mathrm{bar})$ were charged in sequence. The reaction mixture was stirred at $50{ }^{\circ} \mathrm{C}$ (oil bath) for $48 \mathrm{~h}$. The reaction was cooled to room temperature and the pressure was carefully released in a well-ventilated hood. The solution was transferred into a solution of pyridinium chlorochromate (PCC) $(0.4 \mathrm{mmol})$, sodium acetate trihydrate $(0.4 \mathrm{mmol})$, $\mathrm{SiO}_{2}(100 \mathrm{mg})$ in $5 \mathrm{~mL}$ dichloromethane, The reaction mixture was stirred at $25^{\circ} \mathrm{C}$ (oil bath) overnight. The solution was concentrated and the ester was isolated by column chromatography. The enantiomeric excesses of $\mathbf{2 a - 2 t}, \mathbf{4 a - 4}$ fere determined HPLC.<smiles>O=C1C[C@H](c2ccccc2)CO1</smiles>

(S)-4-phenyldihydrofuran-2(3H)-one (2a): colorless oil; isolated yield: 87\%; $90 \%$ ee; $[\alpha]_{\mathrm{D}}^{25}=+39.3\left(\mathrm{c}=1.0, \mathrm{CHCl}_{3}\right)$, ref 3: $[\alpha]_{\mathrm{D}}{ }^{20}=+41.0\left(\mathrm{c}=0.84, \mathrm{CHCl}_{3}, 91 \%\right.$ ee $)$. The enantiomeric excess was determined by HPLC on Chiralpak AD-H column, hexane: isopropanol $=99: 1$; flow rate $=1.0 \mathrm{~mL} / \mathrm{min}$; $\mathrm{UV}$ detection at $220 \mathrm{~nm} ; \mathrm{t}_{\mathrm{R}}=25.3 \mathrm{~min}$ (major), $27.5 \mathrm{~min}$ (minor). ${ }^{1} \mathrm{H} \mathrm{NMR}\left(400 \mathrm{MHz}, \mathrm{CDCl}_{3}\right) \delta$ 7.40-7.36 (m, 2H), 7.33-7.28 (m, 1H), 7.26-7.23 (m, 2H), 4.67 (dd, $J=9.0,7.9 \mathrm{~Hz}, 1 \mathrm{H}), 4.27(\mathrm{dd}, J=9.0,8.1 \mathrm{~Hz}$, $1 \mathrm{H}), 3.84-3.75(\mathrm{~m}, 1 \mathrm{H}), 2.93(\mathrm{dd}, J=17.5,8.7 \mathrm{~Hz}, 1 \mathrm{H}), 2.68(\mathrm{dd}, J=17.5,9.2 \mathrm{~Hz}, 1 \mathrm{H})$ ppm. ${ }^{13} \mathrm{C}$ NMR $\left(101 \mathrm{MHz}, \mathrm{CDCl}_{3}\right) \delta 176.73,139.60,129.36,127.94,126.94,74.30$, 41.31, 35.94 ppm. 
<smiles>Cc1ccc([C@H]2COC(=O)C2)cc1</smiles>

(S)-4-(p-tolyl)dihydrofuran-2(3H)-one (2b): white solid; m. p.: $58-60{ }^{\circ} \mathrm{C}$, isolated yield: $95 \% ; 90 \%$ ee; $[\alpha]_{\mathrm{D}}{ }^{25}=+42.9\left(\mathrm{c}=1.0, \mathrm{CHCl}_{3}\right)$, The enantiomeric excess was determined by HPLC on Chiralpak AD-H column, hexane: isopropanol = 97:3; flow rate $=1.0 \mathrm{~mL} / \mathrm{min}$; $U V$ detection at $220 \mathrm{~nm} ; \mathrm{t}_{\mathrm{R}}=17.4 \mathrm{~min}$ (major), $18.4 \mathrm{~min}$ (minor). ${ }^{1} \mathrm{H}$ NMR (400 MHz, $\left.\mathrm{CDCl}_{3}\right) \delta$ 7.19-7.11 (m, 4H), $4.65(\mathrm{dd}, J=8.9,8.0 \mathrm{~Hz}, 1 \mathrm{H}), 4.24$ $(\mathrm{dd}, J=8.8,8.3 \mathrm{~Hz}, 1 \mathrm{H}), 3.75(\mathrm{~m}, 1 \mathrm{H}), 2.90(\mathrm{dd}, J=17.5,8.7 \mathrm{~Hz}, 1 \mathrm{H}), 2.65(\mathrm{dd}, J=$ 17.5, $9.3 \mathrm{~Hz}, 1 \mathrm{H}), 2.34$ (s, 3H) ppm. ${ }^{13} \mathrm{C} \mathrm{NMR}\left(101 \mathrm{MHz}, \mathrm{CDCl}_{3}\right) \delta 176.84,137.68$, 136.52, 129.99, 126.82, 74.43, 40.99, 36.01, $21.26 \mathrm{ppm}$.<smiles>CC(C)c1ccc([C@H]2COC(=O)C2)cc1</smiles>

(S)-4-(4-isopropylphenyl)dihydrofuran-2(3H)-one (2c): light yellow liquid; isolated yield: $71 \% ; 89 \%$ ee; $[\alpha]_{\mathrm{D}}^{25}=+25.9\left(\mathrm{c}=1.0, \mathrm{CHCl}_{3}\right)$, The enantiomeric excess was determined by HPLC on Chiralpak AD-H column, hexane: isopropanol = 99:1; flow rate $=1.0 \mathrm{~mL} / \mathrm{min}$; $U V$ detection at $220 \mathrm{~nm} ; \mathrm{t}_{\mathrm{R}}=22.5 \mathrm{~min}$ (major), $26.6 \mathrm{~min}$ (minor). ${ }^{1} \mathrm{H}$ NMR $\left(400 \mathrm{MHz}, \mathrm{CDCl}_{3}\right) \delta$ 7.24-7.15 (m, 4H), $4.65(\mathrm{dd}, J=8.9,8.0 \mathrm{~Hz}, 1 \mathrm{H}), 4.28-$ $4.23(\mathrm{~m}, 1 \mathrm{H}), 3.81-3.72(\mathrm{~m}, 1 \mathrm{H}), 2.94-2.87(\mathrm{~m}, 2 \mathrm{H}), 2.67(\mathrm{dd}, J=17.5,9.4 \mathrm{~Hz}, 1 \mathrm{H})$, $1.24(\mathrm{~d}, J=6.9 \mathrm{~Hz}, 6 \mathrm{H}) \mathrm{ppm} .{ }^{13} \mathrm{C} \mathrm{NMR}\left(101 \mathrm{MHz}, \mathrm{CDCl}_{3}\right) \delta 176.87,148.69,136.83$, $127.38,126.93,74.41,41.04,36.03,33.98,24.19$ ppm. HRMS calculated $[\mathrm{M}+\mathrm{Na}]^{+}$for $\mathrm{C}_{13} \mathrm{H}_{16} \mathrm{NaO}_{2}=227.1043$, found: 227.1046 .<smiles>CC(C)(C)c1ccc([C@H]2COC(=O)C2)cc1</smiles>

(S)-4-(4-(tert-butyl)phenyl)dihydrofuran-2(3H)-one (2d): white solid; m. p.: 93$96^{\circ} \mathrm{C}$, isolated yield: $87 \% ; 88 \%$ ee; $[\alpha]_{\mathrm{D}}{ }^{25}=+24.3\left(\mathrm{c}=1.0, \mathrm{CHCl}_{3}\right)$, The enantiomeric excess was determined by HPLC on Chiralpak AD-H column, hexane: isopropanol = 99:1; flow rate $=1.0 \mathrm{~mL} / \mathrm{min}$; $U V$ detection at $220 \mathrm{~nm}$; $t_{\mathrm{R}}=21.2 \mathrm{~min}$ (major), $29.6 \mathrm{~min}$ (minor). ${ }^{1} \mathrm{H}$ NMR (400 MHz, $\left.\mathrm{CDCl}_{3}\right) \delta$ 7.41-7.38 (m, 2H), 7.19-7.17 (m, 2H), 4.65 (dd, $J=8.8,8.1 \mathrm{~Hz}, 1 \mathrm{H}), 4.28-4.24(\mathrm{~m}, 1 \mathrm{H}), 3.81-3.73(\mathrm{~m}, 1 \mathrm{H}), 2.91(\mathrm{dd}, J=17.5,8.7 \mathrm{~Hz}$, $1 \mathrm{H}), 2.68(\mathrm{dd}, J=17.5,9.4 \mathrm{~Hz}, 1 \mathrm{H}), 1.32(\mathrm{~s}, 9 \mathrm{H}) \mathrm{ppm} .{ }^{13} \mathrm{C} \mathrm{NMR}\left(101 \mathrm{MHz}, \mathrm{CDCl}_{3}\right) \delta$ $176.87,150.97,136.45,126.68,126.25,74.39,40.95,36.01,34.77,31.52 \mathrm{ppm}$. 
<smiles>O=C1C[C@H](c2ccc(-c3ccccc3)cc2)CO1</smiles>

(S)-4-([1,1'-biphenyl]-4-yl)dihydrofuran-2(3H)-one (2e): white solid; m. p.: 184$187^{\circ} \mathrm{C}$, isolated yield: $71 \% ; 85 \%$ ee; $[\alpha]_{\mathrm{D}}{ }^{25}=+38.8\left(\mathrm{c}=1.0, \mathrm{CHCl}_{3}\right)$, The enantiomeric excess was determined by HPLC on Chiralpak AD-H column, hexane: isopropanol = 90:10; flow rate $=1.0 \mathrm{~mL} / \mathrm{min}$; $\mathrm{UV}$ detection at $254 \mathrm{~nm} ; \mathrm{t}_{\mathrm{R}}=17.2 \mathrm{~min}$ (major), 20.2 min (minor). ${ }^{1} \mathrm{H}$ NMR $\left(400 \mathrm{MHz}, \mathrm{CDCl}_{3}\right) \delta$ 7.60-7.29 (m, 9H), 4.69 (dd, $J=8.9,8.0$ $\mathrm{Hz}, 1 \mathrm{H}), 4.30$ (dd, $J=9.0,8.1 \mathrm{~Hz}, 1 \mathrm{H}), 3.87-3.78$ (m, 1H), 2.95 (dd, $J=17.5,8.7 \mathrm{~Hz}$, $1 \mathrm{H}), 2.71(\mathrm{dd}, J=17.5,9.1 \mathrm{~Hz}, 1 \mathrm{H}) \mathrm{ppm} .{ }^{13} \mathrm{C} \mathrm{NMR}\left(101 \mathrm{MHz}, \mathrm{CDCl}_{3}\right) \delta 176.68,140.93$, $140.55,138.58,129.11,128.04,127.79,127.41,127.26,74.27,41.04,35.97$ ppm.<smiles>COc1ccc([C@H]2COC(=O)C2)cc1</smiles>

(S)-4-(4-methoxyphenyl)dihydrofuran-2(3H)-one (2f): white solid; m. p.: 86-91 ${ }^{\circ} \mathrm{C}$, isolated yield: $88 \%$; $90 \%$ ee; $[\alpha]_{\mathrm{D}}{ }^{25}=+39.3\left(\mathrm{c}=1.0, \mathrm{CHCl}_{3}\right)$, The enantiomeric excess was determined by HPLC on Chiralpak AD-H column, hexane: isopropanol = 99:1; flow rate $=1.0 \mathrm{~mL} / \mathrm{min}$; $\mathrm{UV}$ detection at $220 \mathrm{~nm}$; $\mathrm{t}_{\mathrm{R}}=44.7 \mathrm{~min}$ (major), $48.3 \mathrm{~min}$ (minor). ${ }^{1} \mathrm{H}$ NMR $\left(400 \mathrm{MHz}, \mathrm{CDCl}_{3}\right) \delta$ 7.18-7.14 (m, 2H), 6.92-6.88 (m, 2H), $4.64(\mathrm{dd}$, $J=8.9,7.9 \mathrm{~Hz}, 1 \mathrm{H}), 4.22(\mathrm{dd}, J=8.9,8.2 \mathrm{~Hz}, 1 \mathrm{H}), 3.81(\mathrm{~s}, 3 \mathrm{H}), 3.79-3.70(\mathrm{~m}, 1 \mathrm{H})$, $2.90(\mathrm{dd}, J=17.5,8.6 \mathrm{~Hz}, 1 \mathrm{H}), 2.64(\mathrm{dd}, J=17.5,9.3 \mathrm{~Hz}, 1 \mathrm{H}) \mathrm{ppm} .{ }^{13} \mathrm{C}$ NMR $(101$ $\left.\mathrm{MHz}, \mathrm{CDCl}_{3}\right) \delta 176.85,159.22,131.45,128.00,114.67,74.53,55.57,40.67,36.12 \mathrm{ppm}$.<smiles>O=C1C[C@H](c2ccc(C(F)(F)F)cc2)CO1</smiles>

(S)-4-(4-(trifluoromethyl)phenyl)dihydrofuran-2(3H)-one (2g): light yellow oil; isolated yield: $72 \%$; $93 \%$ ee; $[\alpha]_{\mathrm{D}}{ }^{25}=+16.0\left(\mathrm{c}=1.0, \mathrm{CHCl}_{3}\right)$, The enantiomeric excess was determined by HPLC on Chiralpak AD-H column, hexane: isopropanol = 99:1; flow rate $=1.0 \mathrm{~mL} / \mathrm{min}$; $\mathrm{UV}$ detection at $220 \mathrm{~nm} ; \mathrm{t}_{\mathrm{R}}=46.3 \mathrm{~min}$ (major), $49.0 \mathrm{~min}$ (minor). ${ }^{1} \mathrm{H}$ NMR (400 MHz, $\left.\mathrm{CDCl}_{3}\right) \delta 7.64(\mathrm{~d}, J=8.2 \mathrm{~Hz}, 2 \mathrm{H}), 7.38(\mathrm{~d}, J=8.1 \mathrm{~Hz}$, $2 \mathrm{H}), 4.71(\mathrm{dd}, J=9.0,7.9 \mathrm{~Hz}, 1 \mathrm{H}), 4.29(\mathrm{dd}, J=9.1,7.6 \mathrm{~Hz}, 1 \mathrm{H}), 3.91-3.83(\mathrm{~m}, 1 \mathrm{H})$, $2.99(\mathrm{dd}, J=17.5,8.8 \mathrm{~Hz}, 1 \mathrm{H}), 2.68(\mathrm{dd}, J=17.5,8.6 \mathrm{~Hz}, 1 \mathrm{H}) \mathrm{ppm} .{ }^{13} \mathrm{C}$ NMR $(101$ $\left.\mathrm{MHz}, \mathrm{CDCl}_{3}\right) \delta 176.08,143.85,130.35(\mathrm{q}, J=32.5 \mathrm{~Hz}), 127.42,126.41(\mathrm{q}, J=3.7 \mathrm{~Hz})$, $124.12(\mathrm{q}, J=272.1 \mathrm{~Hz}), 73.78,41.10,35.78 \mathrm{ppm}$. 
<smiles>CCOC(=O)c1ccc([C@H]2COC(=O)C2)cc1</smiles>

ethyl (S)-4-(5-oxotetrahydrofuran-3-yl)benzoate (2h): white solid; m. p.: 77-81 ${ }^{\circ} \mathrm{C}$, isolated yield: $80 \% ; 86 \%$ ee; $[\alpha]_{\mathrm{D}}{ }^{25}=+26.3\left(\mathrm{c}=1.0, \mathrm{CHCl}_{3}\right)$, The enantiomeric excess was determined by HPLC on Chiralpak AD-H column, hexane: isopropanol = 90:10; flow rate $=1.0 \mathrm{~mL} / \mathrm{min}$; UV detection at $220 \mathrm{~nm} ; \mathrm{t}_{\mathrm{R}}=22.1 \mathrm{~min}$ (major), $25.0 \mathrm{~min}$ (minor). ${ }^{1} \mathrm{H} \mathrm{NMR}\left(400 \mathrm{MHz}, \mathrm{CDCl}_{3}\right) \delta$ 8.06-8.04 (m, 2H), 7.33-7.31 (m, 2H), 4.70 (dd, $J=9.1,7.9 \mathrm{~Hz}, 1 \mathrm{H}), 4.38(\mathrm{q}, J=7.1 \mathrm{~Hz}, 2 \mathrm{H}), 4.29(\mathrm{dd}, J=9.1,7.7 \mathrm{~Hz}, 1 \mathrm{H}), 3.91-3.83$ $(\mathrm{m}, 1 \mathrm{H}), 2.98(\mathrm{dd}, J=17.5,8.8 \mathrm{~Hz}, 1 \mathrm{H}), 2.70(\mathrm{dd}, J=17.5,8.8 \mathrm{~Hz}, 1 \mathrm{H}), 1.40(\mathrm{t}, J=$ $7.1 \mathrm{~Hz}, 3 \mathrm{H}) \mathrm{ppm} .{ }^{13} \mathrm{C} \mathrm{NMR}\left(101 \mathrm{MHz}, \mathrm{CDCl}_{3}\right) \delta 176.23,166.27,144.69,130.59$, $130.20,126.97,73.80,61.37,41.21,35.73,14.54 \mathrm{ppm}$.<smiles>O=C1C[C@H](c2ccc(F)cc2)CO1</smiles>

(S)-4-(4-fluorophenyl)dihydrofuran-2(3H)-one (2i): colorless oil; isolated yield: $78 \% ; 92 \%$ ee; $[\alpha]_{\mathrm{D}}^{25}=+26.2\left(\mathrm{c}=1.0, \mathrm{CHCl}_{3}\right)$, The enantiomeric excess was determined by HPLC on Chiralpak AD-H column, hexane: isopropanol $=99: 1$; flow rate $=1.0$ $\mathrm{mL} / \mathrm{min}$; UV detection at $220 \mathrm{~nm}$; $\mathrm{t}_{\mathrm{R}}=46.7 \mathrm{~min}$ (major), $49.8 \mathrm{~min}$ (minor). ${ }^{1} \mathrm{H} \mathrm{NMR}$ $\left(400 \mathrm{MHz}, \mathrm{CDCl}_{3}\right) \delta$ 7.24-7.19 (m, 2H), 7.09-7.04 (m, 2H), $4.67(\mathrm{dd}, J=9.1,7.8 \mathrm{~Hz}$, $1 \mathrm{H}), 4.24(\mathrm{dd}, J=9.1,7.8 \mathrm{~Hz}, 1 \mathrm{H}), 3.83-3.75(\mathrm{~m}, 1 \mathrm{H}), 2.94(\mathrm{dd}, J=17.5,8.7 \mathrm{~Hz}, 1 \mathrm{H})$, $2.64(\mathrm{dd}, J=17.5,9.0 \mathrm{~Hz}, 1 \mathrm{H}) \mathrm{ppm} .{ }^{13} \mathrm{C} \mathrm{NMR}\left(101 \mathrm{MHz}, \mathrm{CDCl}_{3}\right) \delta 176.46,162.35(\mathrm{~d}$, $J=246.6 \mathrm{~Hz}), 135.40(\mathrm{~d}, J=3.3 \mathrm{~Hz}), 128.54(\mathrm{~d}, J=8.1 \mathrm{~Hz}), 116.29(\mathrm{~d}, J=21.5 \mathrm{~Hz})$, $74.27,40.68,36.07 \mathrm{ppm}$.<smiles>O=C1C[C@H](c2ccc(Cl)cc2)CO1</smiles>

(S)-4-(4-chlorophenyl)dihydrofuran-2(3H)-one (2j): white solid; m. p.: $61-63{ }^{\circ} \mathrm{C}$, isolated yield: $73 \%$; $90 \%$ ee; $[\alpha]_{\mathrm{D}}{ }^{25}=+27.5\left(\mathrm{c}=1.0, \mathrm{CHCl}_{3}\right)$, The enantiomeric excess was determined by HPLC on Chiralpak AD-H column, hexane: isopropanol = 99:1; flow rate $=1.0 \mathrm{~mL} / \mathrm{min}$; UV detection at $220 \mathrm{~nm}$; $\mathrm{t}_{\mathrm{R}}=41.7 \mathrm{~min}$ (major), $44.6 \mathrm{~min}$ (minor). ${ }^{1} \mathrm{H}$ NMR $\left(400 \mathrm{MHz}, \mathrm{CDCl}_{3}\right) \delta$ 7.36-7.33 (m,2H), 7.19-7.17 (m, 2H), $4.66(\mathrm{dd}$, $J=9.0,7.9 \mathrm{~Hz}, 1 \mathrm{H}), 4.24(\mathrm{dd}, J=9.1,7.8 \mathrm{~Hz}, 1 \mathrm{H}), 3.82-3.74(\mathrm{~m}, 1 \mathrm{H}), 2.94(\mathrm{dd}, J=$ $17.5,8.7 \mathrm{~Hz}, 1 \mathrm{H}), 2.63(\mathrm{dd}, J=17.5,8.9 \mathrm{~Hz}, 1 \mathrm{H}) \mathrm{ppm} .{ }^{13} \mathrm{C} \mathrm{NMR}\left(101 \mathrm{MHz}, \mathrm{CDCl}_{3}\right) \delta$ $176.32,138.18,133.79,129.54,128.32,74.03,40.75,35.89$ ppm. 
<smiles>O=C1C[C@H](c2cccc(F)c2)CO1</smiles>

(S)-4-(3-fluorophenyl)dihydrofuran-2(3H)-one (2k): light yellow oil; isolated yield: $79 \% ; 87 \%$ ee; $[\alpha]_{\mathrm{D}}^{25}=+23.6\left(\mathrm{c}=1.0, \mathrm{CHCl}_{3}\right)$, The enantiomeric excess was determined by HPLC on Chiralpak AD-H column, hexane: isopropanol $=99: 1$; flow rate $=1.0$ $\mathrm{mL} / \mathrm{min}$; UV detection at $220 \mathrm{~nm}$; $\mathrm{t}_{\mathrm{R}}=41.4 \mathrm{~min}$ (major), $47.8 \mathrm{~min}$ (minor). ${ }^{1} \mathrm{H}$ NMR $\left(400 \mathrm{MHz}, \mathrm{CDCl}_{3}\right) \delta$ 7.37-7.32 (m, 1H), $7.04-6.94(\mathrm{~m}, 3 \mathrm{H}), 4.67(\mathrm{dd}, J=9.0,7.9 \mathrm{~Hz}$, $1 \mathrm{H}), 4.27(\mathrm{dd}, J=9.1,7.8 \mathrm{~Hz}, 1 \mathrm{H}), 3.84-3.76(\mathrm{~m}, 1 \mathrm{H}), 2.95(\mathrm{dd}, J=17.5,8.7 \mathrm{~Hz}, 1 \mathrm{H})$, $2.66(\mathrm{dd}, J=17.5,8.8 \mathrm{~Hz}, 1 \mathrm{H}) \mathrm{ppm} .{ }^{13} \mathrm{C} \mathrm{NMR}\left(101 \mathrm{MHz}, \mathrm{CDCl}_{3}\right) \delta 176.24,163.33(\mathrm{~d}$, $J=247.3 \mathrm{~Hz}), 142.23(\mathrm{~d}, J=7.0 \mathrm{~Hz}), 131.04(\mathrm{~d}, J=8.4 \mathrm{~Hz}), 122.58(\mathrm{~d}, J=2.9 \mathrm{~Hz})$, $114.94(\mathrm{~d}, J=21.0 \mathrm{~Hz}), 114.09(\mathrm{~d}, J=21.9 \mathrm{~Hz}), 73.88,41.02(\mathrm{~d}, J=1.7 \mathrm{~Hz}), 35.78$ ppm. HRMS calculated $[\mathrm{M}+\mathrm{Na}]^{+}$for $\mathrm{C}_{10} \mathrm{H}_{9} \mathrm{FNaO}_{2}=203.0479$, found: 203.0503.<smiles>Cc1ccccc1[C@H]1COC(=O)C1</smiles>

(S)-4-(o-tolyl)dihydrofuran-2(3H)-one (2I): colorless oil; isolated yield: $94 \%$; $90 \%$ ee; $[\alpha]_{\mathrm{D}}{ }^{25}=+35.5\left(\mathrm{c}=1.0, \mathrm{CHCl}_{3}\right)$, The enantiomeric excess was determined by HPLC on Chiralpak AD-H column, hexane: isopropanol $=95: 5$; flow rate $=1.0 \mathrm{~mL} / \mathrm{min}$; UV detection at $220 \mathrm{~nm} ; \mathrm{t}_{\mathrm{R}}=12.6 \mathrm{~min}$ (major), $14.1 \mathrm{~min}$ (minor). ${ }^{1} \mathrm{H} \mathrm{NMR}$ (400 MHz, $\left.\mathrm{CDCl}_{3}\right) \delta$ 7.24-7.19 (m, 4H), $4.65(\mathrm{dd}, J=9.0,7.6 \mathrm{~Hz}, 1 \mathrm{H}), 4.29(\mathrm{dd}, J=9.1,7.0 \mathrm{~Hz}$, $1 \mathrm{H}), 4.03-3.95(\mathrm{~m}, 1 \mathrm{H}), 2.91(\mathrm{dd}, J=17.5,8.7 \mathrm{~Hz}, 1 \mathrm{H}), 2.64(\mathrm{dd}, J=17.5,8.0 \mathrm{~Hz}, 1 \mathrm{H})$, $2.35(\mathrm{~s}, 3 \mathrm{H}) \mathrm{ppm} .{ }^{13} \mathrm{C} \mathrm{NMR}\left(101 \mathrm{MHz}, \mathrm{CDCl}_{3}\right) \delta 176.81,137.84,136.04,131.13$, $127.68,127.10,125.05,73.65,37.27,35.46,19.95 \mathrm{ppm}$.<smiles>Cc1ccc([C@H]2COC(=O)C2)cc1C</smiles>

(S)-4-(3,4-dimethylphenyl)dihydrofuran-2(3H)-one (2m): white solid; m. p.: 33$36{ }^{\circ} \mathrm{C}$, isolated yield: $90 \% ; 90 \%$ ee; $[\alpha]_{\mathrm{D}}^{25}=+15.1\left(\mathrm{c}=1.0, \mathrm{CHCl}_{3}\right)$, The enantiomeric excess was determined by HPLC on Chiralpak AD-H column, hexane: isopropanol = 99:1; flow rate $=1.0 \mathrm{~mL} / \mathrm{min}$; $\mathrm{UV}$ detection at $220 \mathrm{~nm}$; $\mathrm{t}_{\mathrm{R}}=21.8 \mathrm{~min}$ (major), $24.0 \mathrm{~min}$ (minor). ${ }^{1} \mathrm{H}$ NMR $\left(400 \mathrm{MHz}, \mathrm{CDCl}_{3}\right) \delta$ 7.14-6.95 (m, 3H), 4.66-4.62 (m, 1H), 4.26$4.22(\mathrm{~m}, 1 \mathrm{H}), 3.77-3.68(\mathrm{~m}, 1 \mathrm{H}), 2.90(\mathrm{dd}, J=17.5,8.7 \mathrm{~Hz}, 1 \mathrm{H}), 2.66(\mathrm{dd}, J=17.5,9.3$ $\mathrm{Hz}, 1 \mathrm{H}), 2.27(\mathrm{~s}, 3 \mathrm{H}), 2.25(\mathrm{~s}, 3 \mathrm{H}) \mathrm{ppm} .{ }^{13} \mathrm{C} \mathrm{NMR}\left(101 \mathrm{MHz}, \mathrm{CDCl}_{3}\right) \delta 176.96,137.70$, $136.99,136.37,130.51,128.22,124.27,74.52,41.00,36.06,20.10,19.64$ ppm. HRMS calculated $[\mathrm{M}+\mathrm{Na}]^{+}$for $\mathrm{C}_{12} \mathrm{H}_{14} \mathrm{NaO}_{2}=213.0886$, found: 213.0889 . 
<smiles>COc1ccc([C@H]2COC(=O)C2)cc1OC</smiles>

(S)-4-(3,4-dimethoxyphenyl)dihydrofuran-2(3H)-one (2n): white solid; m. p.: 114$115{ }^{\circ} \mathrm{C}$, isolated yield: $74 \% ; 90 \%$ ee; $[\alpha]_{\mathrm{D}}{ }^{25}=+16.6\left(\mathrm{c}=1.0, \mathrm{CHCl}_{3}\right)$, The enantiomeric excess was determined by HPLC on Chiralcel OD-H column, hexane: isopropanol $=85: 15$; flow rate $=1.0 \mathrm{~mL} / \mathrm{min}$; $\mathrm{UV}$ detection at $210 \mathrm{~nm} ; \mathrm{t}_{\mathrm{R}}=39.0 \mathrm{~min}$ (minor), 41.3 min (major) ${ }^{1} \mathrm{H}$ NMR (400 MHz, $\left.\mathrm{CDCl}_{3}\right) \delta 6.87-6.72(\mathrm{~m}, 3 \mathrm{H}), 4.65(\mathrm{dd}, J=8.9,7.9 \mathrm{~Hz}$, $1 \mathrm{H}), 4.26(\mathrm{dd}, J=9.0,8.0 \mathrm{~Hz}, 1 \mathrm{H}), 3.89(\mathrm{~s}, 3 \mathrm{H}), 3.88(\mathrm{~s}, 3 \mathrm{H}), 3.78-3.70(\mathrm{~m}, 1 \mathrm{H}), 2.92$ $(\mathrm{dd}, J=17.5,8.7 \mathrm{~Hz}, 1 \mathrm{H}), 2.66(\mathrm{dd}, J=17.5,9.0 \mathrm{~Hz}, 1 \mathrm{H}) \mathrm{ppm} .{ }^{13} \mathrm{C} \mathrm{NMR}(101 \mathrm{MHz}$, $\left.\mathrm{CDCl}_{3}\right) \delta 176.80,149.60,148.74,132.12,118.94,111.73,110.02,74.51,56.21,56.20$, 41.07, $36.17 \mathrm{ppm}$.<smiles>O=C1C[C@H](c2ccc3c(c2)OCCO3)CO1</smiles>

(S)-4-(2,3-dihydrobenzo[b][1,4]dioxin-6-yl)dihydrofuran-2(3H)-one (2o): yellow solid; m. p.: $101-102{ }^{\circ} \mathrm{C}$, isolated yield: $80 \% ; 88 \%$ ee; $[\alpha]_{\mathrm{D}}^{25}=+36.1\left(\mathrm{c}=1.0, \mathrm{CHCl}_{3}\right)$, The enantiomeric excess was determined by HPLC on Chiralpak AD-H column, hexane: isopropanol $=85: 15$; flow rate $=1.0 \mathrm{~mL} / \mathrm{min}$; $U V$ detection at $220 \mathrm{~nm} ; \mathrm{t}_{\mathrm{R}}=16.4 \mathrm{~min}$ (major), $17.9 \mathrm{~min}$ (minor). ${ }^{1} \mathrm{H} \mathrm{NMR}\left(400 \mathrm{MHz}, \mathrm{CDCl}_{3}\right) \delta 6.85(\mathrm{~d}, J=8.3 \mathrm{~Hz}, 1 \mathrm{H})$, 6.74-6.68 (m, 2H), $4.61(\mathrm{dd}, J=9.0,7.9 \mathrm{~Hz}, 1 \mathrm{H}), 4.27-4.23(\mathrm{~m}, 4 \mathrm{H}), 4.20$ (dd, $J=8.9$, $8.3 \mathrm{~Hz}, 1 \mathrm{H}), 3.73-3.64(\mathrm{~m}, 1 \mathrm{H}), 2.87(\mathrm{dd}, J=17.5,8.6 \mathrm{~Hz}, 1 \mathrm{H}), 2.61$ (dd, $J=17.5,9.4$ $\mathrm{Hz}, 1 \mathrm{H}) \mathrm{ppm} .{ }^{13} \mathrm{C} \mathrm{NMR}\left(101 \mathrm{MHz}, \mathrm{CDCl}_{3}\right) \delta 176.72,144.07,143.24,132.66,119.78$, $118.03,115.73,74.33,64.62,64.55,40.73,35.99 \mathrm{ppm}$. HRMS calculated $[\mathrm{M}+\mathrm{Na}]^{+}$for $\mathrm{C}_{12} \mathrm{H}_{12} \mathrm{NaO}_{4}=243.0628$, found: 243.0635 .<smiles>Cc1cc(C)cc([C@H]2COC(=O)C2)c1</smiles>

(S)-4-(3,5-dimethylphenyl)dihydrofuran-2(3H)-one (2p): colorless oil; isolated yield: $83 \% ; 92 \%$ ee; $[\alpha]_{\mathrm{D}}{ }^{25}=+28.9\left(\mathrm{c}=1.0, \mathrm{CHCl}_{3}\right)$, The enantiomeric excess was determined by HPLC on Chiralpak AD-H column, hexane: isopropanol = 99:1; flow rate $=1.0 \mathrm{~mL} / \mathrm{min}$; $U V$ detection at $220 \mathrm{~nm} ; \mathrm{t}_{\mathrm{R}}=25.4 \mathrm{~min}$ (major), $28.2 \mathrm{~min}$ (minor). ${ }^{1} \mathrm{H}$ NMR $\left(400 \mathrm{MHz}, \mathrm{CDCl}_{3}\right) \delta 6.94(\mathrm{~s}, 1 \mathrm{H}), 6.84(\mathrm{~s}, 2 \mathrm{H}), 4.64(\mathrm{dd}, J=8.9,8.0 \mathrm{~Hz}, 1 \mathrm{H})$, $4.25(\mathrm{dd}, J=8.9,8.1 \mathrm{~Hz}, 1 \mathrm{H}), 3.75-3.67(\mathrm{~m}, 1 \mathrm{H}), 2.89(\mathrm{dd}, J=17.5,8.7 \mathrm{~Hz}, 1 \mathrm{H}), 2.66$ 
$(\mathrm{dd}, J=17.5,9.1 \mathrm{~Hz}, 1 \mathrm{H}), 2.31(\mathrm{~s}, 6 \mathrm{H}) \mathrm{ppm} .{ }^{13} \mathrm{C} \mathrm{NMR}\left(101 \mathrm{MHz}, \mathrm{CDCl}_{3}\right) \delta 176.93$, 139.59, 139.00, 129.50, 124.70, 74.41, 41.18, 35.96, 21.54 ppm. HRMS calculated $[\mathrm{M}+\mathrm{Na}]^{+}$for $\mathrm{C}_{12} \mathrm{H}_{14} \mathrm{NaO}_{2}=213.0886$, found: 213.0915 .<smiles>O=C1C[C@H](c2ccc3ccccc3c2)CO1</smiles>

(S)-4-(naphthalen-2-yl)dihydrofuran-2(3H)-one (2q): white solid; m. p.: 104-106 ${ }^{\circ} \mathrm{C}$, isolated yield: $77 \%$; $90 \%$ ee; $[\alpha]_{\mathrm{D}}{ }^{25}=+37.6\left(\mathrm{c}=1.0, \mathrm{CHCl}_{3}\right)$, The enantiomeric excess was determined by HPLC on Chiralpak AD-H column, hexane: isopropanol = 99:1; flow rate $=1.0 \mathrm{~mL} / \mathrm{min}$; $U V$ detection at $220 \mathrm{~nm} ; t_{R}=42.0 \mathrm{~min}$ (major), $45.7 \mathrm{~min}$ (minor). ${ }^{1} \mathrm{H}$ NMR $\left(400 \mathrm{MHz}, \mathrm{CDCl}_{3}\right) \delta 7.86-7.80(\mathrm{~m}, 3 \mathrm{H}), 7.66(\mathrm{~d}, J=0.8 \mathrm{~Hz}, 1 \mathrm{H})$, 7.53-7.46 (m, 2H), $7.32(\mathrm{dd}, J=8.5,1.9 \mathrm{~Hz}, 1 \mathrm{H}), 4.72(\mathrm{dd}, J=9.1,7.9 \mathrm{~Hz}, 1 \mathrm{H}), 4.36$ (dd, $J=9.1,7.8 \mathrm{~Hz}, 1 \mathrm{H}), 3.97-3.89(\mathrm{~m}, 1 \mathrm{H}), 2.99$ (dd, $J=17.5,8.7 \mathrm{~Hz}, 1 \mathrm{H}), 2.77$ (dd, $J=17.5,8.9 \mathrm{~Hz}, 1 \mathrm{H}) \mathrm{ppm} .{ }^{13} \mathrm{C}$ NMR $\left(101 \mathrm{MHz}, \mathrm{CDCl}_{3}\right) \delta 176.71,136.96,133.60$, $132.91,129.36,127.94,127.91,126.91,126.48,125.75,124.71,74.19,41.42,35.90$ ppm.<smiles>O=C1C[C@H](c2ccco2)CO1</smiles>

(R)-4-(furan-2-yl)dihydrofuran-2(3H)-one (2r): light yellow oil; isolated yield: 75\%; $85 \%$ ee; $[\alpha]_{\mathrm{D}}{ }^{25}=+16.5\left(\mathrm{c}=1.0, \mathrm{CHCl}_{3}\right)$, The enantiomeric excess was determined by HPLC on Chiralpak AD-H column, hexane: isopropanol $=99: 1$; flow rate $=1.0 \mathrm{~mL} / \mathrm{min}$; UV detection at $220 \mathrm{~nm}$; $\mathrm{t}_{\mathrm{R}}=28.2 \mathrm{~min}$ (major), $32.3 \mathrm{~min}$ (minor). ${ }^{1} \mathrm{H} \mathrm{NMR}$ (400 MHz, $\left.\mathrm{CDCl}_{3}\right) \delta 7.38(\mathrm{dd}, J=1.8,0.7 \mathrm{~Hz}, 1 \mathrm{H}), 6.34(\mathrm{dd}, J=3.2,1.9 \mathrm{~Hz}, 1 \mathrm{H}), 6.18(\mathrm{~d}, J=3.3$ $\mathrm{Hz}, 1 \mathrm{H}), 4.58(\mathrm{dd}, J=9.0,7.8 \mathrm{~Hz}, 1 \mathrm{H}), 4.35(\mathrm{dd}, J=9.0,7.3 \mathrm{~Hz}, 1 \mathrm{H}), 3.920-3.82(\mathrm{~m}$, $1 \mathrm{H}), 2.86(\mathrm{dd}, J=17.5,8.7 \mathrm{~Hz}, 1 \mathrm{H}), 2.76(\mathrm{dd}, J=17.5,8.4 \mathrm{~Hz}, 1 \mathrm{H}) \mathrm{ppm} .{ }^{13} \mathrm{C} \mathrm{NMR}$ $\left(101 \mathrm{MHz}, \mathrm{CDCl}_{3}\right) \delta 176.14,152.33,142.70,110.67,106.55,71.76,35.16,33.60 \mathrm{ppm}$.<smiles>O=C1C[C@H](c2ccc3occc3c2)CO1</smiles>

(S)-4-(benzofuran-5-yl)dihydrofuran-2(3H)-one (2s): white solid; m. p.: $97-99{ }^{\circ} \mathrm{C}$, isolated yield: $90 \%$; $90 \%$ ee; $[\alpha]_{\mathrm{D}}{ }^{25}=+44.4\left(\mathrm{c}=1.0, \mathrm{CHCl}_{3}\right)$, The enantiomeric excess was determined by HPLC on Chiralpak AD-H column, hexane: isopropanol = 95:5; flow rate $=1.0 \mathrm{~mL} / \mathrm{min}$; $\mathrm{UV}$ detection at $220 \mathrm{~nm} ; \mathrm{t}_{\mathrm{R}}=21.6 \mathrm{~min}$ (major), $23.1 \mathrm{~min}$ (minor). ${ }^{1} \mathrm{H}$ NMR (400 MHz, $\left.\mathrm{CDCl}_{3}\right) \delta 7.65(\mathrm{~d}, J=2.0 \mathrm{~Hz}, 1 \mathrm{H}), 7.48(\mathrm{dd}, J=9.7,4.7$ 
$\mathrm{Hz}, 2 \mathrm{H}), 7.15(\mathrm{dd}, J=8.5,1.5 \mathrm{~Hz}, 1 \mathrm{H}), 6.76(\mathrm{~d}, J=1.2 \mathrm{~Hz}, 1 \mathrm{H}), 4.72-4.68(\mathrm{~m}, 1 \mathrm{H})$, 4.33-4.28 (m, 1H), 3.93-3.84(m, 1H), $2.97(\mathrm{dd}, J=17.5,8.7 \mathrm{~Hz}, 1 \mathrm{H}), 2.71(\mathrm{dd}, J=17.5$, $9.0 \mathrm{~Hz}, 1 \mathrm{H}) \mathrm{ppm} .{ }^{13} \mathrm{C} \mathrm{NMR}\left(101 \mathrm{MHz}, \mathrm{CDCl}_{3}\right) \delta 176.80,154.47,146.17,134.25$, $128.29,123.18,119.33,112.20,106.68,74.72,41.30,36.41$ ppm. HRMS calculated $[\mathrm{M}+\mathrm{Na}]^{+}$for $\mathrm{C}_{12} \mathrm{H}_{10} \mathrm{NaO}_{3}=225.0522$, found: 225.0522 .<smiles>O=C1C[C@H](c2ccc3sccc3c2)CO1</smiles>

(S)-4-(benzo[b]thiophen-5-yl)dihydrofuran-2(3H)-one (2t): white solid; m. p.: 104$105{ }^{\circ} \mathrm{C}$, isolated yield: $93 \% ; 86 \%$ ee; $[\alpha]_{\mathrm{D}}{ }^{25}=+35.6\left(\mathrm{c}=1.0, \mathrm{CHCl}_{3}\right)$, The enantiomeric excess was determined by HPLC on Chiralpak AD-H column, hexane: isopropanol = 90:10; flow rate $=1.0 \mathrm{~mL} / \mathrm{min}$; $\mathrm{UV}$ detection at $220 \mathrm{~nm} ; \mathrm{t}_{\mathrm{R}}=15.1 \mathrm{~min}$ (major), 16.1 min (minor). ${ }^{1} \mathrm{H}$ NMR (400 MHz, $\left.\mathrm{CDCl}_{3}\right) \delta 7.87(\mathrm{~d}, J=8.3 \mathrm{~Hz}, 1 \mathrm{H}), 7.68(\mathrm{~d}, J=1.2$ $\mathrm{Hz}, 1 \mathrm{H}), 7.49$ (d, $J=5.4 \mathrm{~Hz}, 1 \mathrm{H}), 7.32$ (d, $J=5.4 \mathrm{~Hz}, 1 \mathrm{H}), 7.20(\mathrm{dd}, J=8.3,1.5 \mathrm{~Hz}$, $1 \mathrm{H}), 4.71(\mathrm{dd}, J=8.8,8.1 \mathrm{~Hz}, 1 \mathrm{H}), 4.34-4.30(\mathrm{~m}, 1 \mathrm{H}), 3.94-3.86(\mathrm{~m}, 1 \mathrm{H}), 2.98(\mathrm{dd}, J$ $=17.5,8.7 \mathrm{~Hz}, 1 \mathrm{H}), 2.73(\mathrm{dd}, J=17.5,8.9 \mathrm{~Hz}, 1 \mathrm{H}) \mathrm{ppm} .{ }^{13} \mathrm{C} \mathrm{NMR}\left(101 \mathrm{MHz}, \mathrm{CDCl}_{3}\right)$ $\delta 176.76,140.32,139.17,135.83,127.93,123.83,123.47,123.16,121.75,74.53,41.28$, $36.22 \mathrm{ppm}$. HRMS calculated $[\mathrm{M}+\mathrm{Na}]^{+}$for $\mathrm{C}_{12} \mathrm{H}_{10} \mathrm{NaO}_{2} \mathrm{~S}=241.0294$, found: 241.0300.<smiles>CN1C[C@H](c2ccccc2)CC1=O</smiles>

(S)-1-acetyl-4-phenylpyrrolidin-2-one (4a): white solid; m. p.: $76-78{ }^{\circ} \mathrm{C}$, isolated yield: $64 \% ; 84 \%$ ee; $[\alpha]_{\mathrm{D}}{ }^{25}=-6.5(\mathrm{c}=1.0, \mathrm{MeOH})$, ref $4:[\alpha]_{\mathrm{D}}{ }^{20}=-10.0(\mathrm{c}=1.0, \mathrm{MeOH})$. The enantiomeric excess was determined by HPLC on Chiralpak AD-H column, hexane: isopropanol $=95: 5$; flow rate $=0.5 \mathrm{~mL} / \mathrm{min}$; $U V$ detection at $220 \mathrm{~nm} ; \mathrm{t}_{\mathrm{R}}=31.3 \mathrm{~min}$ (minor), $35.6 \mathrm{~min}$ (major). ${ }^{1} \mathrm{H} \mathrm{NMR}\left(400 \mathrm{MHz}, \mathrm{CDCl}_{3}\right) \delta$ 7.38-7.28 (m, 3H), 7.24-7.22 (m, 2H), 4.29 (dd, $J=11.6,8.1 \mathrm{~Hz}, 1 \mathrm{H}), 3.73(\mathrm{dd}, J=11.6,8.3 \mathrm{~Hz}, 1 \mathrm{H}), 3.61-3.53$ (m, $1 \mathrm{H}), 2.99$ (dd, $J=17.4,8.4 \mathrm{~Hz}, 1 \mathrm{H}), 2.80(\mathrm{dd}, J=17.4,9.7 \mathrm{~Hz}, 1 \mathrm{H}), 2.55$ (s, 3H) ppm. ${ }^{13} \mathrm{C} \mathrm{NMR}\left(101 \mathrm{MHz}, \mathrm{CDCl}_{3}\right) \delta 174.49,171.35,140.67,129.25,127.70,126.87,51.95$, 41.04, 36.25, 25.27 ppm.<smiles>Cc1ccc([C@H]2CC(=O)N(C)C2)cc1</smiles>

(S)-1-acetyl-4-(p-tolyl)pyrrolidin-2-one (4b): white solid; m. p.: 66-67 ${ }^{\circ} \mathrm{C}$, isolated yield: $60 \% ; 83 \%$ ee; $[\alpha]_{\mathrm{D}}^{25}=-11.2(\mathrm{c}=1.0, \mathrm{MeOH})$. The enantiomeric excess was 
determined by HPLC on Chiralpak AD-H column, hexane: isopropanol = 95:5; flow rate $=1.0 \mathrm{~mL} / \mathrm{min}$; $U V$ detection at $220 \mathrm{~nm} ; t_{R}=13.5 \mathrm{~min}$ (minor), $14.6 \mathrm{~min}$ (major). ${ }^{1} \mathrm{H}$ NMR $\left(400 \mathrm{MHz}, \mathrm{CDCl}_{3}\right) \delta$ 7.18-7.11 (m, 4H), 4.27 (dd, $\left.J=11.6,8.1 \mathrm{~Hz}, 1 \mathrm{H}\right), 3.70$ $(\mathrm{dd}, J=11.6,8.4 \mathrm{~Hz}, 1 \mathrm{H}), 3.57-3.49(\mathrm{~m}, 1 \mathrm{H}), 2.96(\mathrm{dd}, J=17.4,8.4 \mathrm{~Hz}, 1 \mathrm{H}), 2.77(\mathrm{dd}$, $J=17.4,9.7 \mathrm{~Hz}, 1 \mathrm{H}), 2.54(\mathrm{~s}, 3 \mathrm{H}), 2.34(\mathrm{~s}, 3 \mathrm{H}) \mathrm{ppm} .{ }^{13} \mathrm{C} \mathrm{NMR}\left(101 \mathrm{MHz}, \mathrm{CDCl}_{3}\right) \delta$ 174.63, 171.38, 137.61, 137.41, 129.90, 126.75, 52.06, 41.16, 35.95, 25.29, 21.26 ppm. HRMS calculated $[\mathrm{M}+\mathrm{Na}]^{+}$for $\mathrm{C}_{13} \mathrm{H}_{15} \mathrm{NNaO}_{2}=240.0995$, found: 240.0998 .<smiles>COc1ccc([C@H]2CC(=O)N(C)C2)cc1</smiles>

(S)-1-acetyl-4-(4-methoxyphenyl)pyrrolidin-2-one (4c): white solid; m. p.: 84-88 ${ }^{\circ} \mathrm{C}$, isolated yield: $64 \% ; 84 \%$ ee; $[\alpha]_{\mathrm{D}}{ }^{25}=-10.3(\mathrm{c}=1.0, \mathrm{MeOH})$. The enantiomeric excess was determined by HPLC on Chiralpak AS-H column, hexane: isopropanol = 90:10; flow rate $=1.0 \mathrm{~mL} / \mathrm{min}$; UV detection at $210 \mathrm{~nm} ; \mathrm{t}_{\mathrm{R}}=41.3 \mathrm{~min}$ (minor), $46.0 \mathrm{~min}$ (major). ${ }^{1} \mathrm{H}$ NMR $\left(400 \mathrm{MHz}, \mathrm{CDCl}_{3}\right) \delta$ 7.16-7.13 (m, 2H), 6.91-6.87 (m, 2H), $4.25(\mathrm{dd}$, $J=11.5,8.1 \mathrm{~Hz}, 1 \mathrm{H}), 3.80(\mathrm{~s}, 3 \mathrm{H}), 3.68(\mathrm{dd}, J=11.6,8.4 \mathrm{~Hz}, 1 \mathrm{H}), 3.56-3.47(\mathrm{~m}, 1 \mathrm{H})$, $2.95(\mathrm{dd}, J=17.3,8.3 \mathrm{~Hz}, 1 \mathrm{H}), 2.75(\mathrm{dd}, J=17.4,9.8 \mathrm{~Hz}, 1 \mathrm{H}), 2.54(\mathrm{~s}, 3 \mathrm{H}) \mathrm{ppm} .{ }^{13} \mathrm{C}$ NMR $\left(101 \mathrm{MHz}, \mathrm{CDCl}_{3}\right) \delta 174.61,171.36,159.09,132.59,127.90,114.60,55.57$, 52.16, 41.26, 35.63, $25.29 \mathrm{ppm}$.<smiles>CN1C[C@H](c2ccc(C(F)(F)F)cc2)CC1=O</smiles>

(S)-1-acetyl-4-(4-(trifluoromethyl)phenyl)pyrrolidin-2-one (4d): white solid; m. p.: 97-98 ${ }^{\circ} \mathrm{C}$, isolated yield: $69 \% ; 80 \%$ ee; $[\alpha]_{\mathrm{D}}{ }^{25}=-10.3(\mathrm{c}=1.0, \mathrm{MeOH})$. The enantiomeric excess was determined by HPLC on Chiralpak AD-H column, hexane: isopropanol $=95: 5$; flow rate $=0.5 \mathrm{~mL} / \mathrm{min}$; $U V$ detection at $220 \mathrm{~nm} ; \mathrm{t}_{\mathrm{R}}=48.4 \mathrm{~min}$ (major), 54.1 min (minor). ${ }^{1} \mathrm{H}$ NMR $\left(400 \mathrm{MHz}, \mathrm{CDCl}_{3}\right) \delta 7.63(\mathrm{~d}, J=8.1 \mathrm{~Hz}, 2 \mathrm{H}), 7.37$ $(\mathrm{d}, J=8.5 \mathrm{~Hz}, 2 \mathrm{H}), 4.32(\mathrm{dd}, J=11.5,8.0 \mathrm{~Hz}, 1 \mathrm{H}), 3.75(\mathrm{dd}, J=11.6,8.1 \mathrm{~Hz}, 1 \mathrm{H})$, 3.69-3.60 (m, 1H), 3.04 (dd, $J=17.4,8.3 \mathrm{~Hz}, 1 \mathrm{H}), 2.80$ (dd, $J=17.4,9.4 \mathrm{~Hz}, 1 \mathrm{H}), 2.55$ (s, 3H) ppm. ${ }^{13} \mathrm{C}$ NMR $\left(101 \mathrm{MHz}, \mathrm{CDCl}_{3}\right) \delta 173.81,171.25,144.78,130.11$ (q, $J=$ $32.5 \mathrm{~Hz}$ ), 127.35, 126.27 (q, $J=3.7 \mathrm{~Hz}$ ), 124.17 (q, $J=270.4 \mathrm{~Hz}), 51.57,40.79,36.06$, 25.23 ppm. HRMS calculated $[\mathrm{M}+\mathrm{Na}]^{+}$for $\mathrm{C}_{13} \mathrm{H}_{12} \mathrm{~F}_{3} \mathrm{NNaO}_{2}=294.0712$, found: 294.0716 . 
<smiles>CN1C[C@H](c2ccc(F)cc2)CC1=O</smiles>

(S)-1-acetyl-4-(4-fluorophenyl)pyrrolidin-2-one (4e): white solid; m. p.: 80-83 ${ }^{\circ} \mathrm{C}$, isolated yield: $63 \% ; 86 \%$ ee; $[\alpha]_{\mathrm{D}}{ }^{25}=-6.1(\mathrm{c}=1.0, \mathrm{MeOH})$. The enantiomeric excess was determined by HPLC on Chiralpak AD-H column, hexane: isopropanol = 95:5; flow rate $=1.0 \mathrm{~mL} / \mathrm{min}$; $\mathrm{UV}$ detection at $220 \mathrm{~nm} ; \mathrm{t}_{\mathrm{R}}=19.6 \mathrm{~min}$ (minor), $24.8 \mathrm{~min}$ (major). ${ }^{1} \mathrm{H}$ NMR (400 MHz, $\left.\mathrm{CDCl}_{3}\right) \delta$ 7.23-7.18 (m, 2H), 7.08-7.03 (m, 2H), 4.27 (dd, $J=11.6,8.1 \mathrm{~Hz}, 1 \mathrm{H}), 3.69$ (dd, $J=11.6,8.3 \mathrm{~Hz}, 1 \mathrm{H}), 3.60-3.52(\mathrm{~m}, 1 \mathrm{H}), 2.98(\mathrm{dd}, J=$ 17.4, 8.4 Hz, 1H), $2.76(\mathrm{dd}, J=17.4,9.6 \mathrm{~Hz}, 1 \mathrm{H}), 2.54(\mathrm{~s}, 3 \mathrm{H}) \mathrm{ppm} .{ }^{13} \mathrm{C}$ NMR $(101$ $\left.\mathrm{MHz}, \mathrm{CDCl}_{3}\right) \delta 174.19,171.28,162.22(\mathrm{~d}, J=246.2 \mathrm{~Hz}), 136.39(\mathrm{~d}, J=3.3 \mathrm{~Hz}), 128.42$ $(\mathrm{d}, J=8.1 \mathrm{~Hz}), 116.13(\mathrm{~d}, J=21.4 \mathrm{~Hz}), 51.98,41.12,35.62,25.24$ ppm. HRMS calculated $[\mathrm{M}+\mathrm{Na}]^{+}$for $\mathrm{C}_{12} \mathrm{H}_{12} \mathrm{FNNaO}_{2}=244.0744$, found: 244.0750 .<smiles>CN1C[C@H](c2ccc(Cl)cc2)CC1=O</smiles>

(S)-1-acetyl-4-(4-chlorophenyl)pyrrolidin-2-one (4f): white solid; m. p.: 98-100 ${ }^{\circ} \mathrm{C}$, isolated yield: $60 \% ; 82 \%$ ee; $[\alpha]_{\mathrm{D}}{ }^{25}=-13.9(\mathrm{c}=1.0, \mathrm{MeOH})$. The enantiomeric excess was determined by HPLC on Chiralpak AD-H column, hexane: isopropanol = 95:5; flow rate $=1.0 \mathrm{~mL} / \mathrm{min}$; UV detection at $220 \mathrm{~nm} ; \mathrm{t}_{\mathrm{R}}=44.9 \mathrm{~min}$ (minor), $52.7 \mathrm{~min}$ (major). ${ }^{1} \mathrm{H}$ NMR $\left(400 \mathrm{MHz}, \mathrm{CDCl}_{3}\right) \delta$ 7.35-7.31 (m, 2H), 7.18-7.16 (m, 2H), 4.27 (dd, $J=11.6,8.1 \mathrm{~Hz}, 1 \mathrm{H}), 3.69(\mathrm{dd}, J=11.6,8.2 \mathrm{~Hz}, 1 \mathrm{H}), 3.59-3.51(\mathrm{~m}, 1 \mathrm{H}), 2.99(\mathrm{dd}, J=$ 17.4, 8.4 Hz, 1H), 2.75 (dd, $J=17.4,9.5 \mathrm{~Hz}, 1 \mathrm{H}), 2.54$ (s, 3H) ppm. ${ }^{13} \mathrm{C}$ NMR $(101$ $\left.\mathrm{MHz}, \mathrm{CDCl}_{3}\right) \delta 174.05,171.25,139.16,133.52,129.40,128.24,51.77,40.93,35.70$, $25.24 \mathrm{ppm}$.

\section{Procedures for gram-scale asymmetric hydroformylation of $1 p$}<smiles>C=C(CO)c1cc(C)cc(C)c1</smiles>

10

$8 \mathrm{mmol}, 1.30 \mathrm{~g}$

\section{$\frac{\mathrm{Rh}(\mathrm{acac})(\mathrm{CO})_{2}(1 \mathrm{~mol} \%), \mathrm{L} 2(3 \mathrm{~mol} \%)}{\mathrm{CO} / \mathrm{H}_{2}=3 / 3 \text { bar, toluene, } 50{ }^{\circ} \mathrm{C}, 96 \mathrm{~h}}$}

Gram-scale transformation of $\mathbf{1 p}$ :

A stock solution was made by mixing $(S, S)$-DTBM-YanPhos $(0.24 \mathrm{mmol})$ with $\mathrm{Rh}(\mathrm{acac})(\mathrm{CO})_{2}(0.08 \mathrm{mmol})$ in toluene $(10 \mathrm{~mL})$ at room temperature for $10 \mathrm{~min}$ in a nitrogen-filled glovebox. Then, the catalyst solution was transferred by syringe into the 
vial charged with substrate 1p $(8 \mathrm{mmol}, 1.30 \mathrm{~g})$. The vial was transferred into an autoclave and taken out of the glovebox. Carbon monoxide ( 3 bar) and hydrogen ( 3 bar) were charged in sequence. The reaction mixture was stirred at $50{ }^{\circ} \mathrm{C} \mathrm{(oil} \mathrm{bath)} \mathrm{for} 96 \mathrm{~h}$. The reaction was cooled and the pressure was carefully released in a well-ventilated hood. The solution was concentrated and $\mathbf{5}$ was isolated by column chromatography. Isolated yield: $81 \%, 1.25 \mathrm{~g}, 90 \% \mathrm{ee}, \mathrm{dr}=2: 1$. The ee value was detected after a oxidation reaction with PCC. The diastereoselectivity determined by ${ }^{13} \mathrm{C}$ NMR analysis. HRMS calculated $[\mathrm{M}+\mathrm{Na}]^{+}$for $\mathrm{C}_{12} \mathrm{H}_{16} \mathrm{NaO}_{2}=215.1043$, found: 215.1038 .

\section{Synthesis of the 3-(3,5-dimethylphenyl)tetrahydrofuran 6a}<smiles>Cc1cc(C)cc([C@H]2CO[C@H](O)C2)c1</smiles>

5

$90 \%$ ee, $\mathrm{dr}=2: 1$

\section{$\underset{\mathrm{Et}_{3} \mathrm{SiH}, \mathrm{BF}_{3} \cdot \mathrm{Et}_{2} \mathrm{O}, \mathrm{CH}_{2} \mathrm{Cl}_{2}, 0{ }^{\circ} \mathrm{C}, 24 \mathrm{~h}}{\longrightarrow}$}

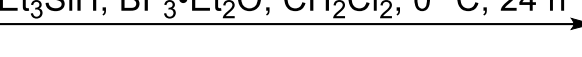<smiles>Cc1cc(C)cc([C@H]2CCOC2)c1</smiles>

$6 a$

$71 \%$ yield, $90 \%$ ee

6a was prepared according to the literature. ${ }^{5}$ To a $10 \mathrm{ml}$ tube was added the chiral hemiacetal 5 (0.2 mmol, 38.4mg, 90\% ee) and dry $\mathrm{CH}_{2} \mathrm{Cl}_{2}(2 \mathrm{~mL})$ and the vial was cooled to $0{ }^{\circ} \mathrm{C}$. To the resulting solution was added triethyl silane $(96 \mu \mathrm{L}, 0.6 \mathrm{mmol})$ followed by $\mathrm{BF}_{3} \cdot \mathrm{Et}_{2} \mathrm{O}(0.6 \mathrm{mmol}, 85.2 \mathrm{mg})$ dropwise at $0{ }^{\circ} \mathrm{C}$. The solution was allowed to stirred at $0{ }^{\circ} \mathrm{C}$ for 24 hours. The organic solvents were removed under reduced pressure and the residue was purified by flash chromatography to give the desired product $6 \mathbf{a}(25 \mathrm{mg}, 71 \%$ yield $)$ with $90 \%$ ee. $[\alpha]_{\mathrm{D}}{ }^{25}=+13.0\left(\mathrm{c}=1.0, \mathrm{CHCl}_{3}\right) .{ }^{1} \mathrm{H} \mathrm{NMR}$ $\left(400 \mathrm{MHz}, \mathrm{CDCl}_{3}\right) \delta 6.87(\mathrm{~s}, 3 \mathrm{H}), 4.13-4.09(\mathrm{~m}, 1 \mathrm{H}), 4.07-4.03(\mathrm{~m}, 1 \mathrm{H}), 3.93-3.87(\mathrm{~m}$, $1 \mathrm{H}), 3.73-3.69(\mathrm{~m}, 1 \mathrm{H}), 3.37-3.29(\mathrm{~m}, 1 \mathrm{H}), 2.37-2.32(\mathrm{~m}, 1 \mathrm{H}), 2.30(\mathrm{~d}, J=0.4 \mathrm{~Hz}, 6 \mathrm{H})$, 2.04-1.95 (m, 1H) ppm. ${ }^{13} \mathrm{C} \mathrm{NMR}\left(101 \mathrm{MHz}, \mathrm{CDCl}_{3}\right) \delta 142.82,138.36,128.40,125.33$, $74.95,68.82,45.12,34.90,21.58 \mathrm{ppm}$. The enantiomeric excess was determined by HPLC on Chiralpak AS-H column, hexane: isopropanol $=99: 1$; flow rate $=0.3 \mathrm{~mL} / \mathrm{min}$; $\mathrm{UV}$ detection at $220 \mathrm{~nm} ; \mathrm{t}_{\mathrm{R}}=23.9 \mathrm{~min}$ (minor), $28.0 \mathrm{~min}$ (major). HRMS calculated $[\mathrm{M}+\mathrm{H}]^{+}$for $\mathrm{C}_{12} \mathrm{H}_{17} \mathrm{O}=177.1274$, found: 177.1258 .

\section{Synthesis of the (4S)-2-allyl-4-(3,5-dimethylphenyl)tetrahydrofuran $6 \mathrm{~b}$}


<smiles>Cc1cc(C)cc([C@H]2CO[C@H](O)C2)c1</smiles>

5

$90 \%$ ee, $\mathrm{dr}=2: 1$

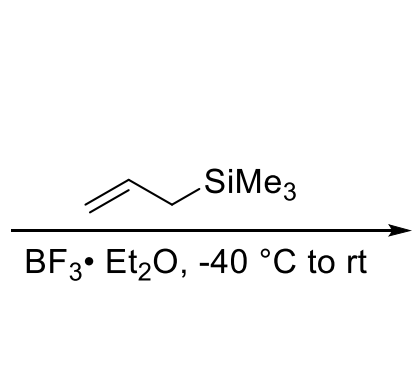

$\mathrm{BF}_{3} \cdot \mathrm{Et}_{2} \mathrm{O},-40^{\circ} \mathrm{C}$ to rt

6b

$88 \%$ yield

$90 \%$ ee, $d r=13: 1$

6b was prepared according to the literature. ${ }^{6}$ To a $10 \mathrm{ml}$ tube was added the chiral hemiacetal 5 (0.2 mmol, 38.4mg, 90\% ee) and dry $\mathrm{CH}_{2} \mathrm{Cl}_{2}(2 \mathrm{~mL})$ and the vial was cooled to $-40{ }^{\circ} \mathrm{C}$. To the resulting solution was added allyltrimethylsilane $(0.6 \mathrm{mmol}$, $68.6 \mathrm{mg})$ followed by $\mathrm{BF}_{3} \cdot \mathrm{Et}_{2} \mathrm{O}(0.6 \mathrm{mmol}, 85.2 \mathrm{mg})$ dropwise at $-40{ }^{\circ} \mathrm{C}$. The solution was allowed to warm to $0{ }^{\circ} \mathrm{C}$ and stirred for $10 \mathrm{~min}$. The organic solvents were removed under reduced pressure and the residue was purified by flash chromatography to give the desired product $6 \mathbf{b}(38.2 \mathrm{mg}, 88 \%$ yield $)$ with $90 \%$ ee and $13: 1 \mathrm{dr}$. $[\alpha]_{\mathrm{D}}{ }^{25}=+14.4$ $\left(\mathrm{c}=1.0, \mathrm{CHCl}_{3}\right)$. The diastereoselectivity determined by ${ }^{13} \mathrm{C} \mathrm{NMR}$ analysis. For the major isomer: ${ }^{1} \mathrm{H} \mathrm{NMR}\left(400 \mathrm{MHz}, \mathrm{CDCl}_{3}\right) \delta 6.86(\mathrm{~s}, 3 \mathrm{H}), 5.91-5.81(\mathrm{~m}, 1 \mathrm{H}), 5.16-5.07$ (m, 2H), 4.24-4.20 (m, 2H), $3.70(\mathrm{t}, \mathrm{J}=8.3 \mathrm{~Hz}, 1 \mathrm{H}), 3.40-3.32(\mathrm{~m}, 1 \mathrm{H}), 2.44-2.36(\mathrm{~m}$, 1H), 2.34-2.33 (m, 1H), $2.30(\mathrm{~s}, 6 \mathrm{H}), 2.12-2.00(\mathrm{~m}, 2 \mathrm{H}) \mathrm{ppm} .{ }^{13} \mathrm{C}$ NMR $(101 \mathrm{MHz}$, $\left.\mathrm{CDCl}_{3}\right) \delta 142.68,138.32,135.12,128.37,125.35,117.29,78.96,74.96,44.65,40.74$, 39.30, $21.57 \mathrm{ppm}$. The enantiomeric excess was determined by HPLC on Chiralpak OJ$\mathrm{H}$ column, hexane: isopropanol $=99: 1$; flow rate $=0.5 \mathrm{~mL} / \mathrm{min}$; $\mathrm{UV}$ detection at 220 $\mathrm{nm} ; \mathrm{t}_{\mathrm{R}}=9.3 \mathrm{~min}$ (major), $10.1 \mathrm{~min}$ (minor). HRMS calculated $[\mathrm{M}+\mathrm{Na}]^{+}$for $\mathrm{C}_{15} \mathrm{H}_{20} \mathrm{NaO}$ $=239.1406$ found: 239.1437 .

\section{Synthesis of the (S)-2-(3,5-dimethylphenyl)butane-1,4-diol 6c}<smiles>Cc1cc(C)cc([C@H]2CO[C@H](O)C2)c1</smiles>

5

$90 \%$ ee, $\mathrm{dr}=2: 1$

\section{$\stackrel{\mathrm{NaBH}_{4}, \mathrm{MeOH}, 0^{\circ} \mathrm{C}, 2 \mathrm{~h}}{\longrightarrow}$}

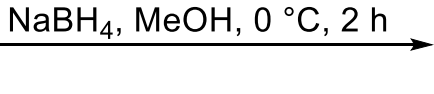

To a $10 \mathrm{ml}$ tube was added $\mathrm{NaBH}_{4}(50 \mathrm{mg})$ and methanol $(2 \mathrm{~mL})$, and the chiral hemiacetal $5\left(0.2 \mathrm{mmol}, 38.4 \mathrm{mg}, 90 \%\right.$ ee) was added dropwise at $0{ }^{\circ} \mathrm{C}$. The solution was allowed to stirred at $0{ }^{\circ} \mathrm{C}$ for 2 hours. The organic solvents were removed under reduced pressure and the residue was purified by flash chromatography to give the desired product $6 \mathbf{c}(38.8 \mathrm{mg}, 99 \%$ yield $)$ with $90 \%$ ee. $[\alpha]_{\mathrm{D}}{ }^{25}=+20.5\left(\mathrm{c}=1.0, \mathrm{CHCl}_{3}\right)$. ${ }^{1} \mathrm{H}$ NMR $\left(400 \mathrm{MHz}, \mathrm{CDCl}_{3}\right) \delta 6.86(\mathrm{~s}, 1 \mathrm{H}), 6.80(\mathrm{~s}, 2 \mathrm{H}), 3.71-3.65(\mathrm{~m}, 3 \mathrm{H}), 3.54(\mathrm{~s}$, 1H), 2.87-2.82 (m, 2H), $2.28(\mathrm{~s}, 6 \mathrm{H}), 1.98(\mathrm{dd}, J=13.5,7.2 \mathrm{~Hz}, 1 \mathrm{H}), 1.83$ (dd, $J=13.3$, $7.6 \mathrm{~Hz}, 1 \mathrm{H}) \mathrm{ppm} .{ }^{13} \mathrm{C} \mathrm{NMR}\left(101 \mathrm{MHz}, \mathrm{CDCl}_{3}\right) \delta 142.46,138.37,128.69,125.82,67.77$, $61.36,46.05,36.10,21.56 \mathrm{ppm}$. The enantiomeric excess was determined by HPLC on 
Chiralpak AS-H column, hexane: isopropanol $=90: 10$; flow rate $=1.0 \mathrm{~mL} / \mathrm{min}$; UV detection at $220 \mathrm{~nm} ; \mathrm{t}_{\mathrm{R}}=8.4 \mathrm{~min}$ (major), $9.1 \mathrm{~min}$ (minor). HRMS calculated $[\mathrm{M}+\mathrm{Na}]^{+}$ for $\mathrm{C}_{12} \mathrm{H}_{18} \mathrm{NaO}_{2}=217.1199$ found: 217.1195 .

\section{General procedure for the AHF of I with $(S, S)$-DTB-YanPhos}

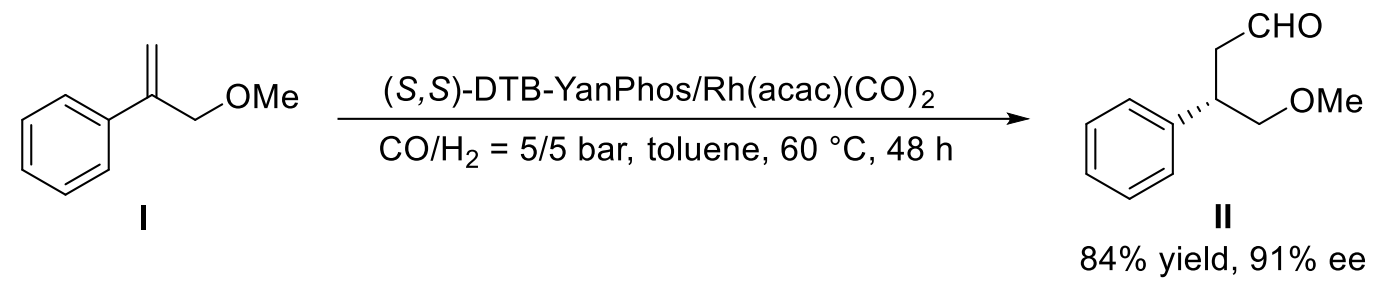

In a glovebox filled with nitrogen, to a $5 \mathrm{ml}$ vial equipped with a magnetic bar was added ligand $(S, S)$-DTB-YanPhos $(0.024 \mathrm{mmol})$ and $\mathrm{Rh}(\mathrm{acac})(\mathrm{CO})_{2}(0.008 \mathrm{mmol}$ in 1 $\mathrm{mL}$ toluene). After stirring for $10 \mathrm{~min}$, the mixture was charged to $\mathbf{I}(0.2 \mathrm{mmol})$. The vial was transferred into an autoclave and taken out of the glovebox. Carbon monoxide ( 5 bar) and hydrogen ( 5 bar) were charged in sequence. The reaction mixture was stirred at $60{ }^{\circ} \mathrm{C}$ (oil bath) for $48 \mathrm{~h}$. The reaction was cooled and the pressure was carefully released in a well-ventilated hood. The organic solvents were removed under reduced pressure and the residue was purified by flash chromatography to give the desired product II (30.0 $\mathrm{mg}, 84 \%$ yield) with $91 \%$ ee. The ee value was detected after a reduction reaction with $\mathrm{NaBH}_{4} .[\alpha]_{\mathrm{D}}{ }^{25}=+36.7\left(\mathrm{c}=1.0, \mathrm{CHCl}_{3}\right) .{ }^{1} \mathrm{H} \mathrm{NMR}(400 \mathrm{MHz}$, $\left.\mathrm{CDCl}_{3}\right) \delta 9.72(\mathrm{t}, J=2.0 \mathrm{~Hz}, 1 \mathrm{H}), 7.33-7.22(\mathrm{~m}, 5 \mathrm{H}), 3.59-3.43(\mathrm{~m}, 3 \mathrm{H}), 3.32(\mathrm{~s}, 3 \mathrm{H})$, 2.90 (ddd, $J=16.8,6.8,2.2 \mathrm{~Hz}, 1 \mathrm{H}), 2.72$ (ddd, $J=16.8,6.9,1.7 \mathrm{~Hz}, 1 \mathrm{H}) \mathrm{ppm} .{ }^{13} \mathrm{C}$ NMR $\left(101 \mathrm{MHz}, \mathrm{CDCl}_{3}\right) \delta 201.71,141.23,128.95,127.88,127.30,77.00,59.06,47.39$, $40.57 \mathrm{ppm}$. The enantiomeric excess was determined by HPLC on Chiralpak AS-H column, hexane: isopropanol $=97: 10$; flow rate $=1.0 \mathrm{~mL} / \mathrm{min} ; \mathrm{UV}$ detection at $210 \mathrm{~nm}$; $\mathrm{t}_{\mathrm{R}}=25.9 \mathrm{~min}$ (major), $32.8 \mathrm{~min}$ (minor).

\section{References}

(1) Ganss, S.; Breit, B. Enantioselective Rhodium-Catalyzed Atom-Economical Macrolactonization. Angew. Chem. Int. Ed. 2016, 55, 9738-9742.

(2) Wei, B.; Chen, C.; You, C.; Lv, H.; Zhang, X. Efficient Synthesis of $(S, R)$-BnYanphos and $\mathrm{Rh} /(S, R)$-Bn-Yanphos Catalyzed Asymmetric Hydroformylation of Vinyl Heteroarenes. Org. Chem. Front. 2017, 4, 288-291.

(3) Zhou, L.; Liu, X.; Ji, J.; Zhang, Y.; Hu, X.; Lin, L.; Feng, X. Enantioselective Baeyer-Villiger Oxidation: Desymmetrization of Meso Cyclic Ketones and Kinetic Resolution of Racemic 2-Arylcyclohexanones. J. Am. Chem. Soc. 2012, 134, 17023-17026. 
(4) Vorona, M.; Orlova, N.; Kuznetsov, E.; Vikainis, S.; Liepinsh, E.; Belyakov, S.; Mishnev, A.; Veinberg, G. Method for the Preparation of 4-Aryl-3-pyrrolin-2-ones and Their 5-Bromo Derivatives. Chem. Heterocycl. Comp. 2013, 49, 1118-1127.

(5) Balha, M.; Mondal, B.; Sahoo, S.; Pan, S. Organocatalytic Asymmetric MichaelHemiacetalization Reaction Between 2-Hydroxyacetophenones and Enals: a Route to Chiral $\beta, \gamma$-Disubstituted $\gamma$-Butyrolactones. J. Org. Chem. 2017, 82, 6409-6416.

(6) Chen, C.; Jin, S.; Zhang, Z.; Wei, B.; Wang, H.; Zhang, K.; Lv, H.; Dong, X. Q.; Zhang, X. Rhodium/Yanphos-Catalyzed Asymmetric Interrupted Intramolecular Hydroaminomethylation of trans-1,2-Disubstituted Alkenes. J. Am. Chem. Soc. 2016, 138, 9017-9020. 


\section{NMR spectra}<smiles>O=C1C[C@H](c2ccccc2)CO1</smiles>

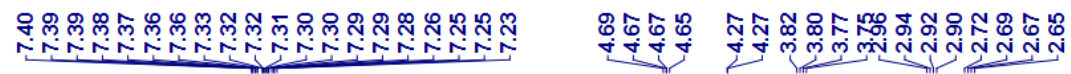
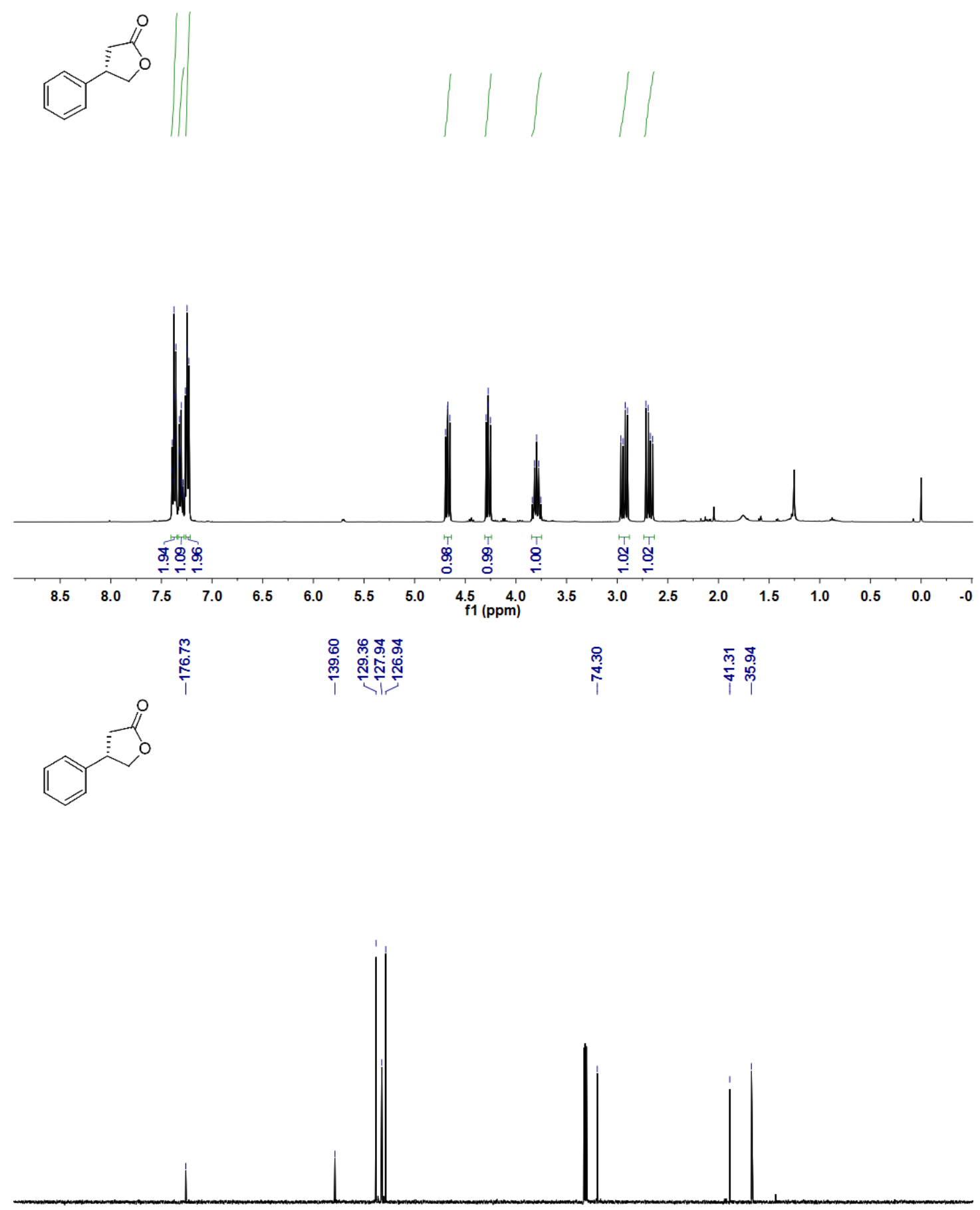

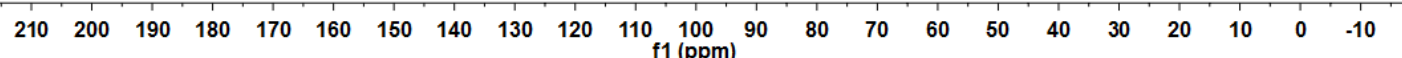



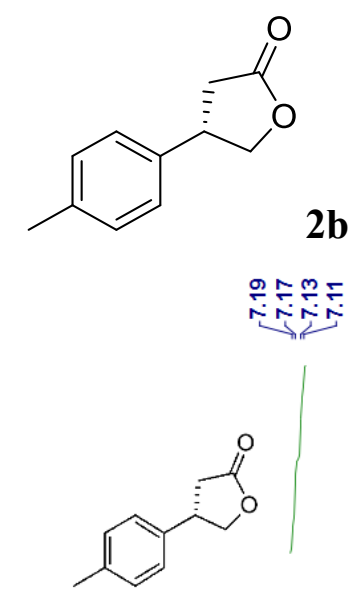

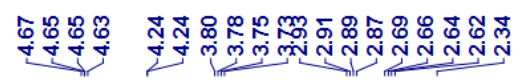
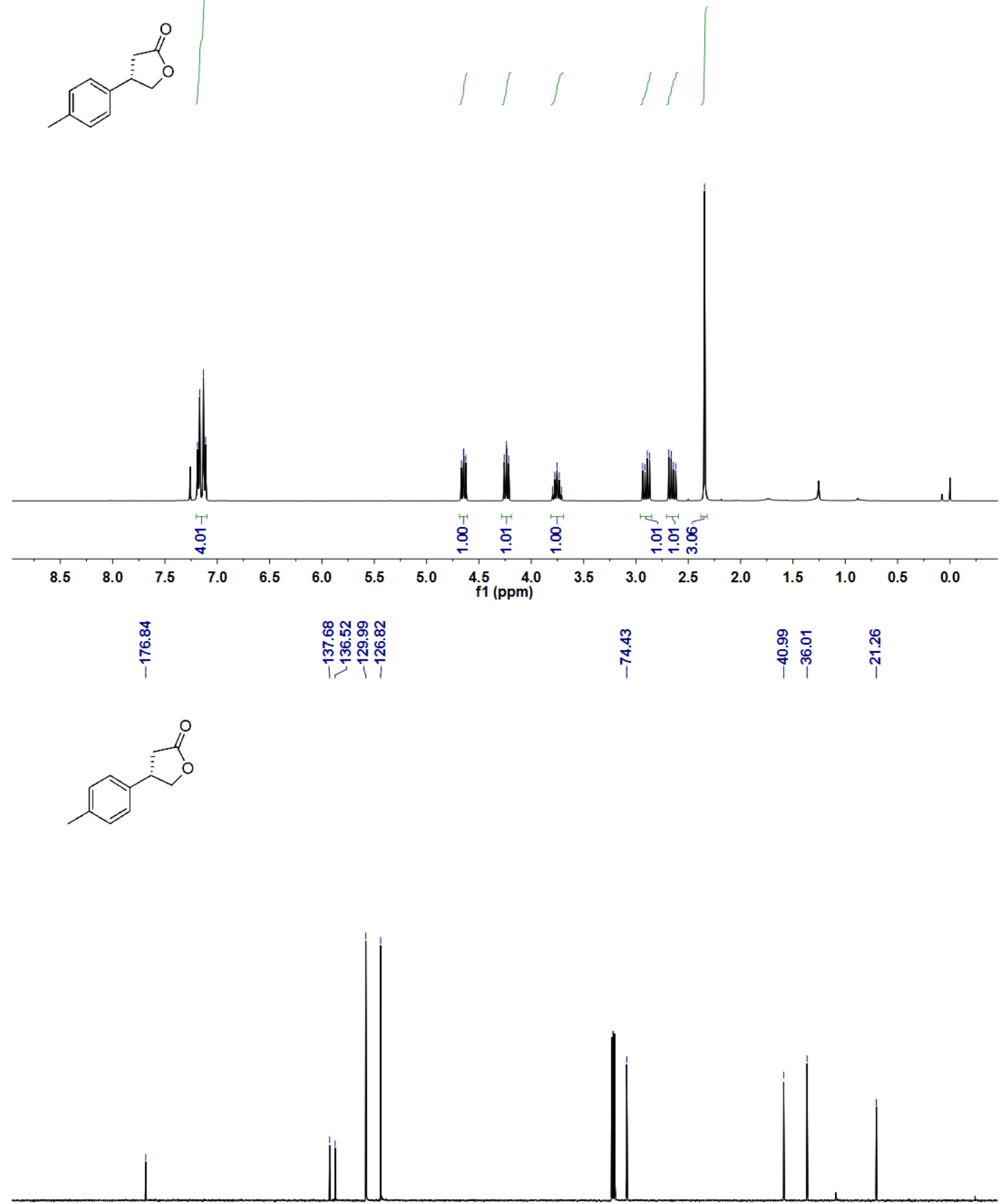

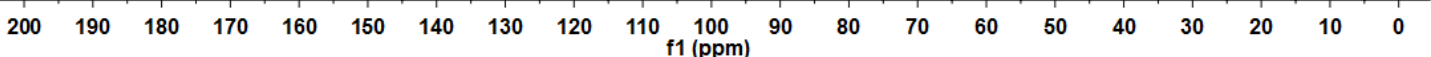


$\overbrace{2 c}^{2 c}$

ำ ำ

近证
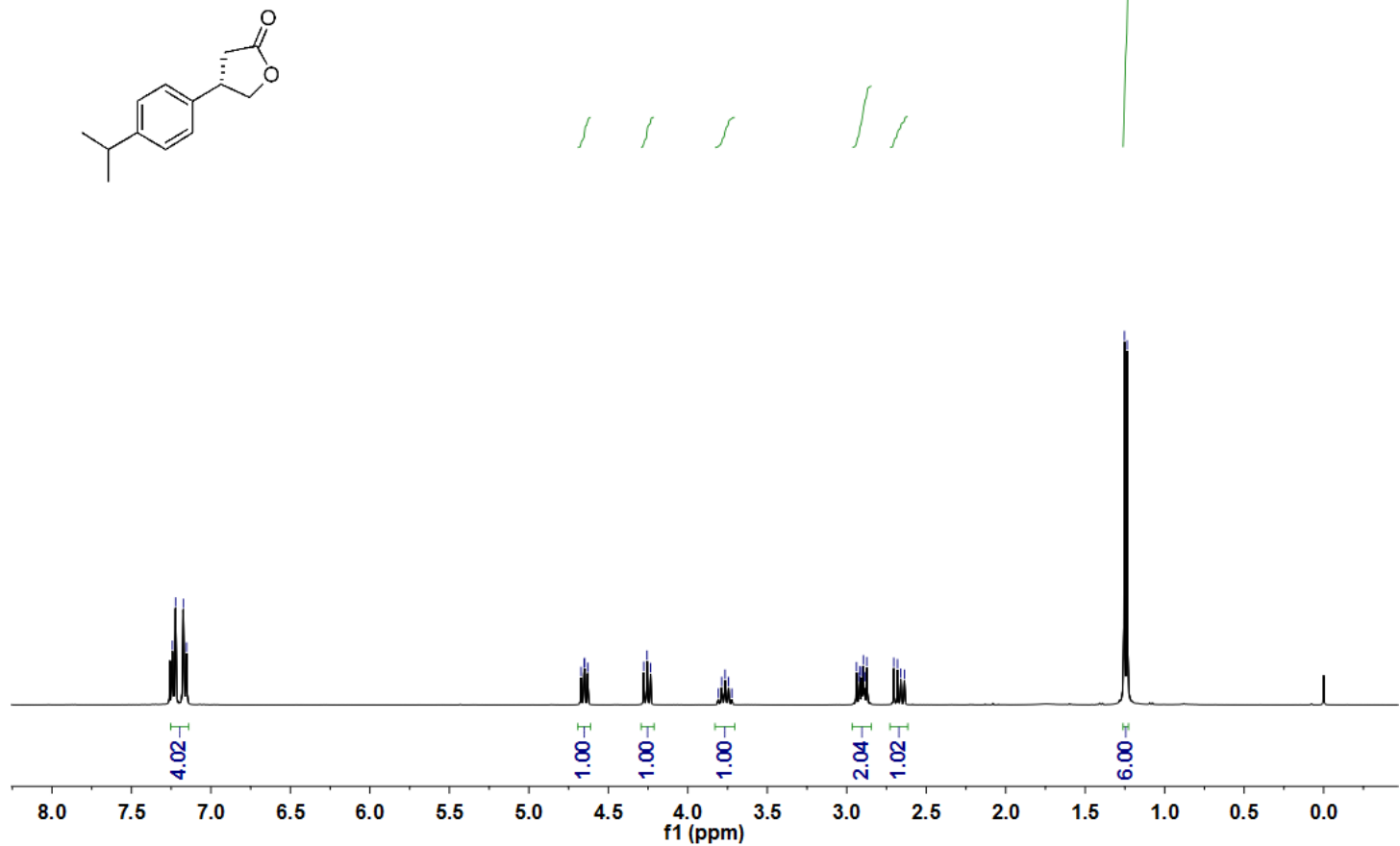

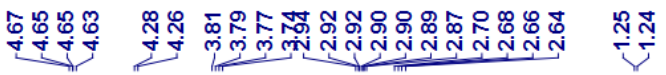

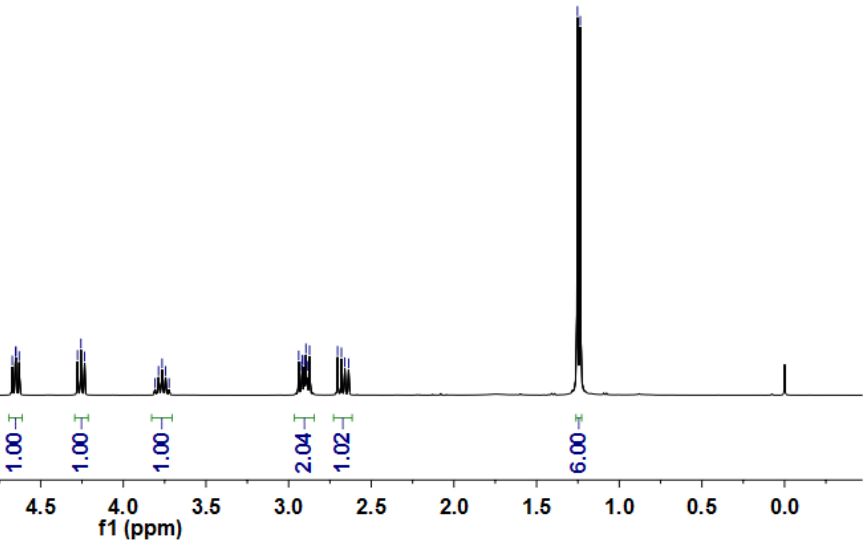

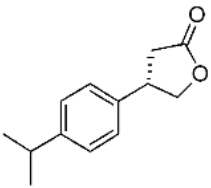

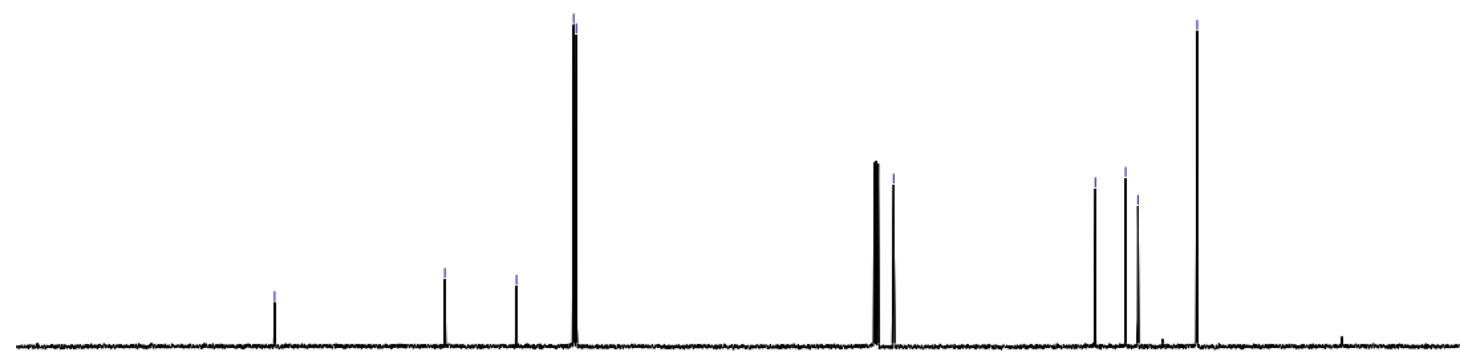

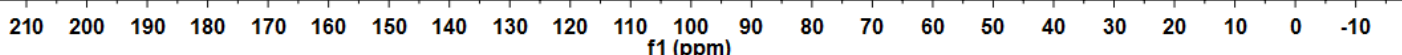


<smiles>CC(C)(C)c1ccc([C@H]2COC(=O)C2)cc1</smiles>

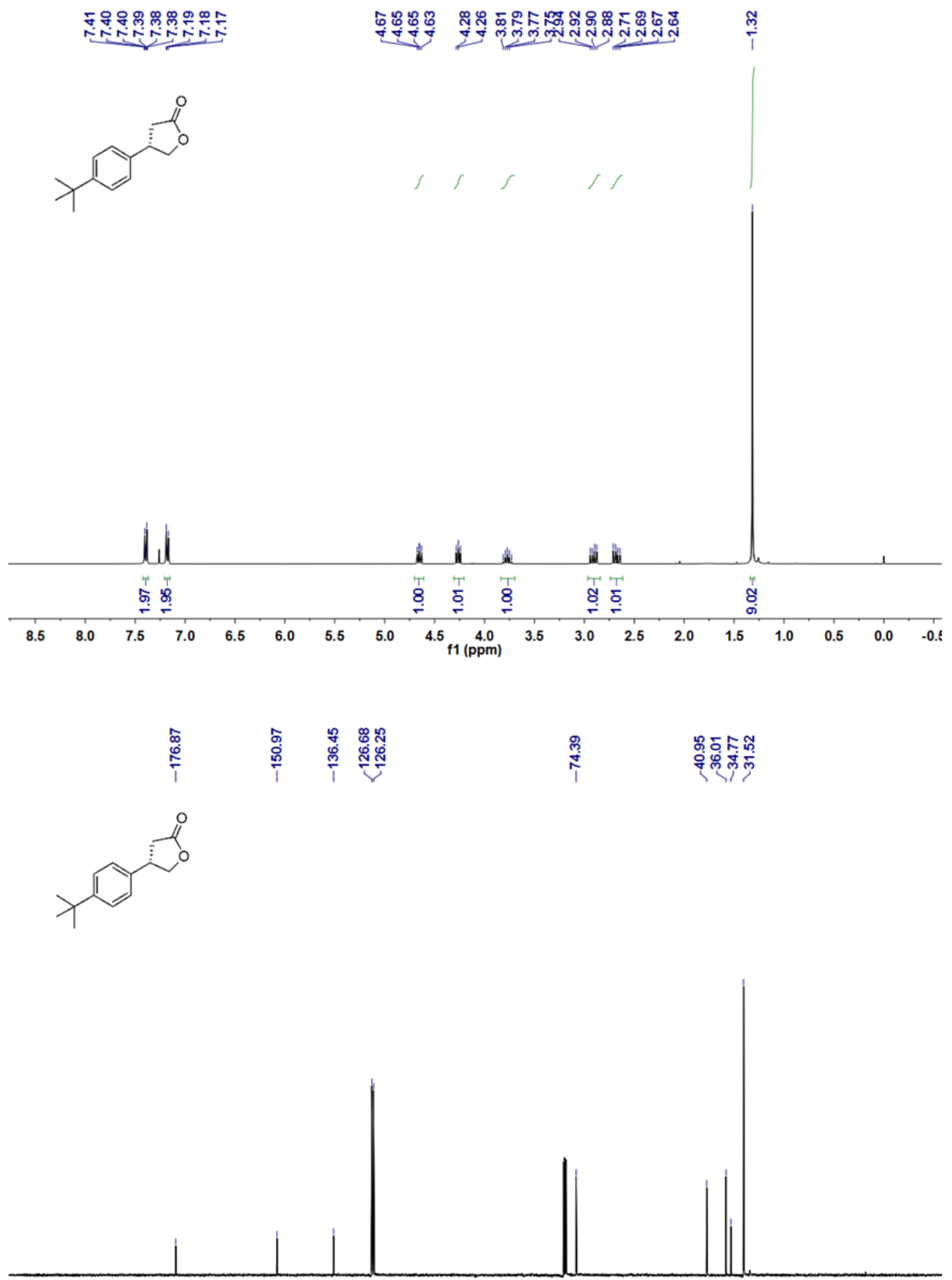

$\begin{array}{lllllllllllllllllllllll}210 & 200 & 190 & 180 & 170 & 160 & 150 & 140 & 130 & 120 & 110 & 100 & 90 & 80 & 70 & 60 & 50 & 40 & 30 & 20 & 10 & 0 & -10\end{array}$ 
<smiles>O=C1C[C@H](c2ccc(-c3ccccc3)cc2)CO1</smiles>

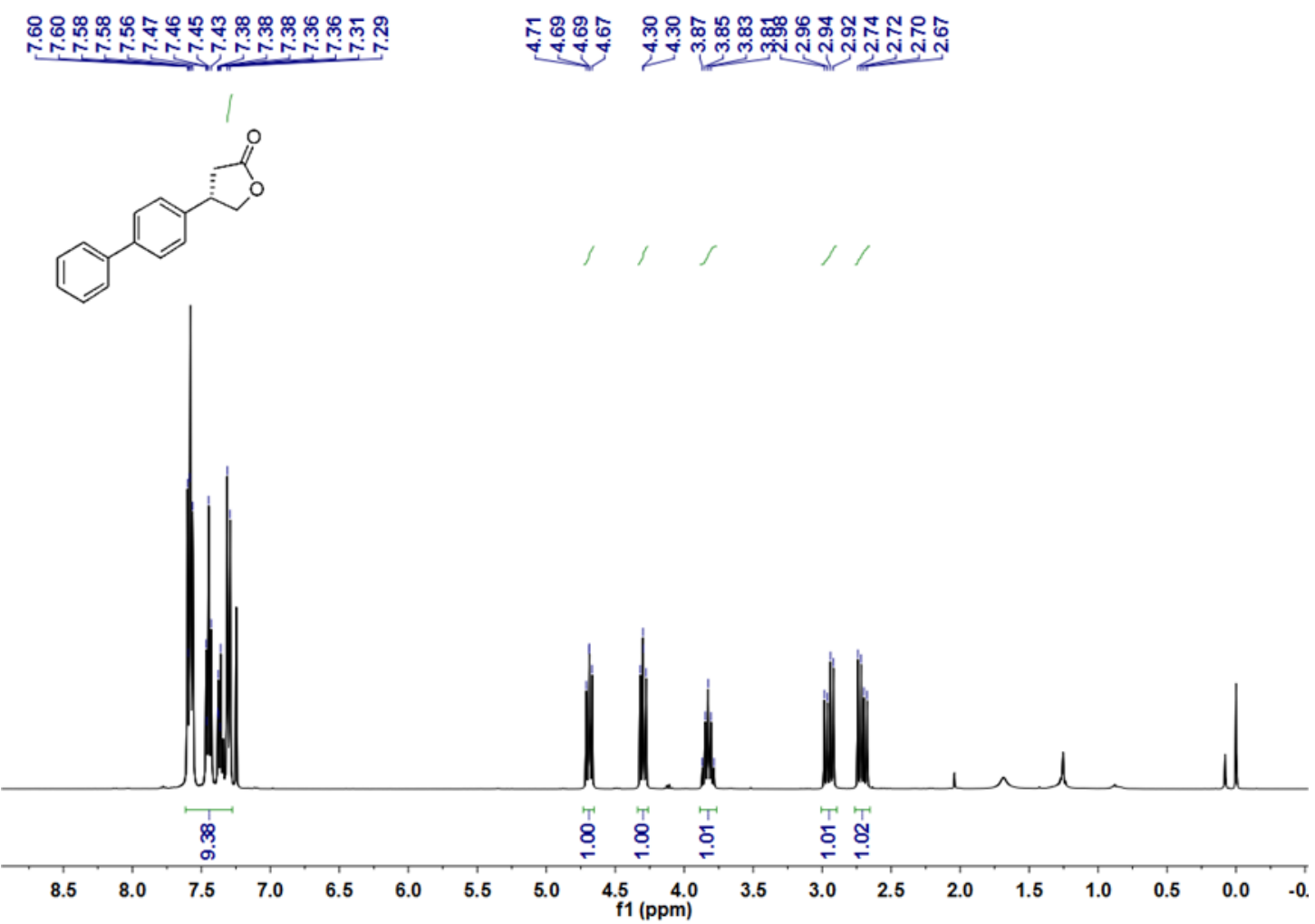

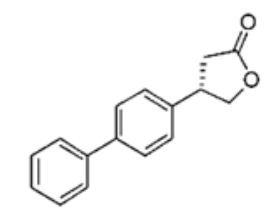

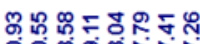

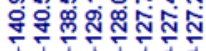

官

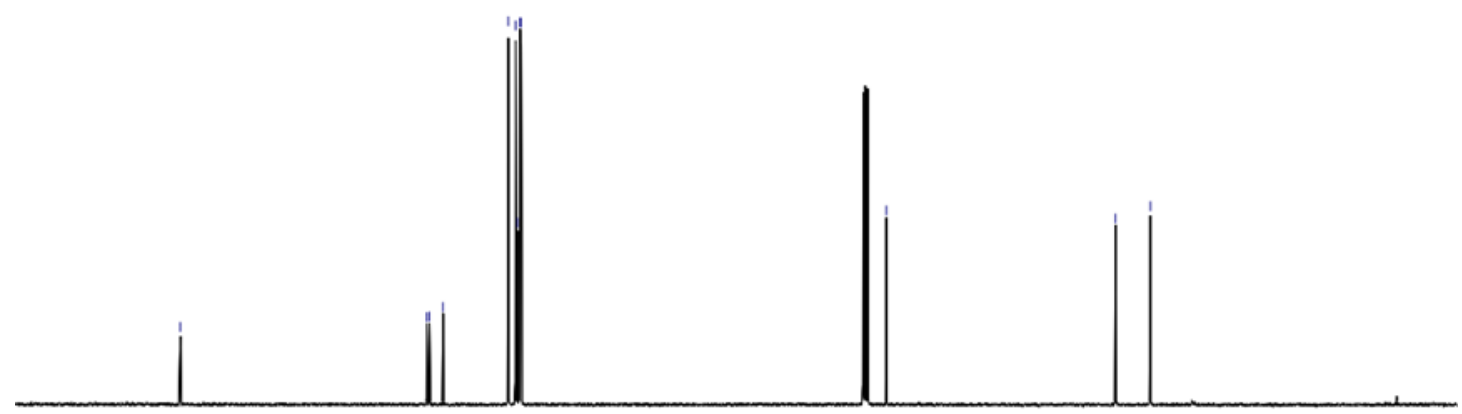

$\begin{array}{llllllllllllllllllll}00 & 190 & 180 & 170 & 160 & 150 & 140 & 130 & 120 & 110 & \begin{array}{l}100 \\ \mathrm{f} 1(\mathrm{ppm})\end{array} & 80 & 70 & 60 & 50 & 40 & 30 & 20 & 10 & 0\end{array}$ 
<smiles>COc1ccc([C@H]2COC(=O)C2)cc1</smiles>

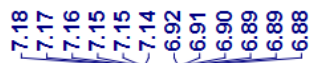

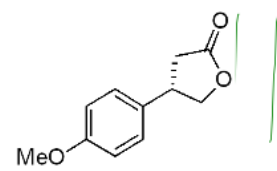

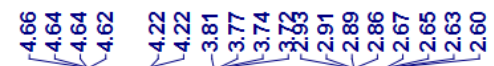

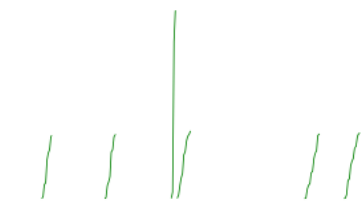

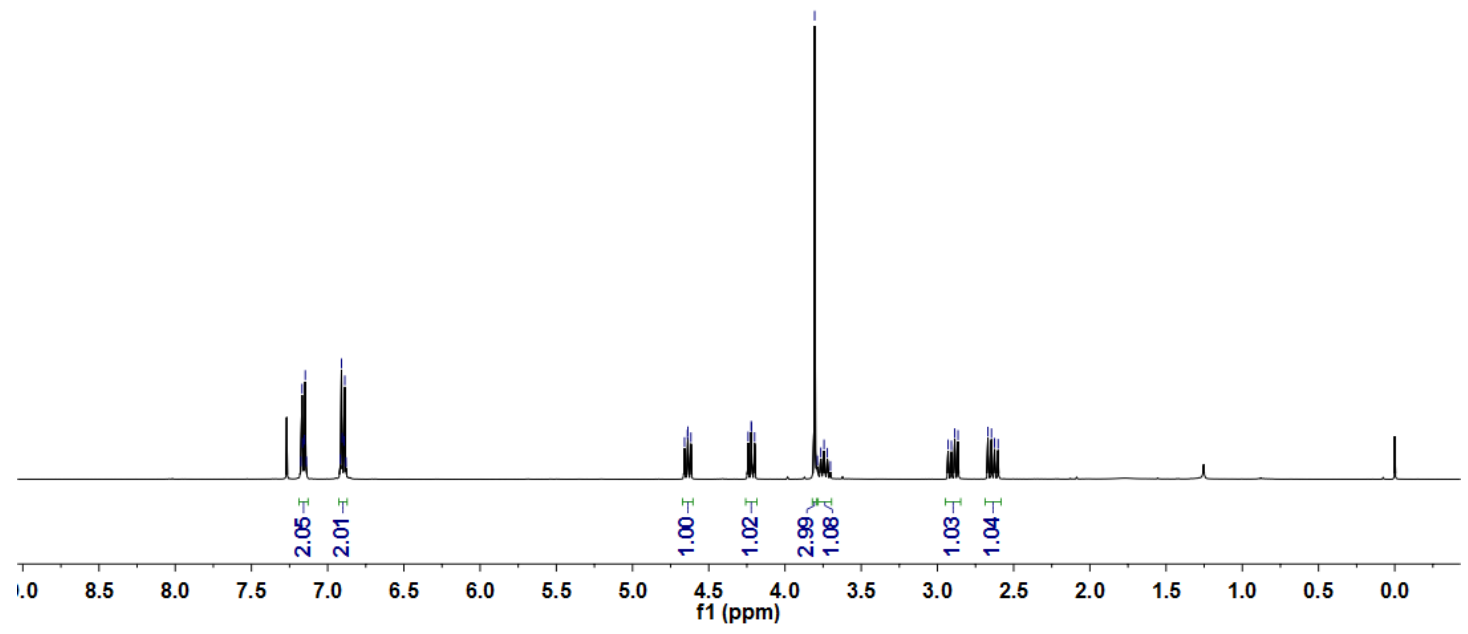

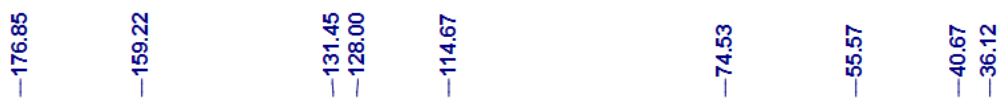<smiles>COc1ccc(CC2COC(=O)C2)cc1</smiles>

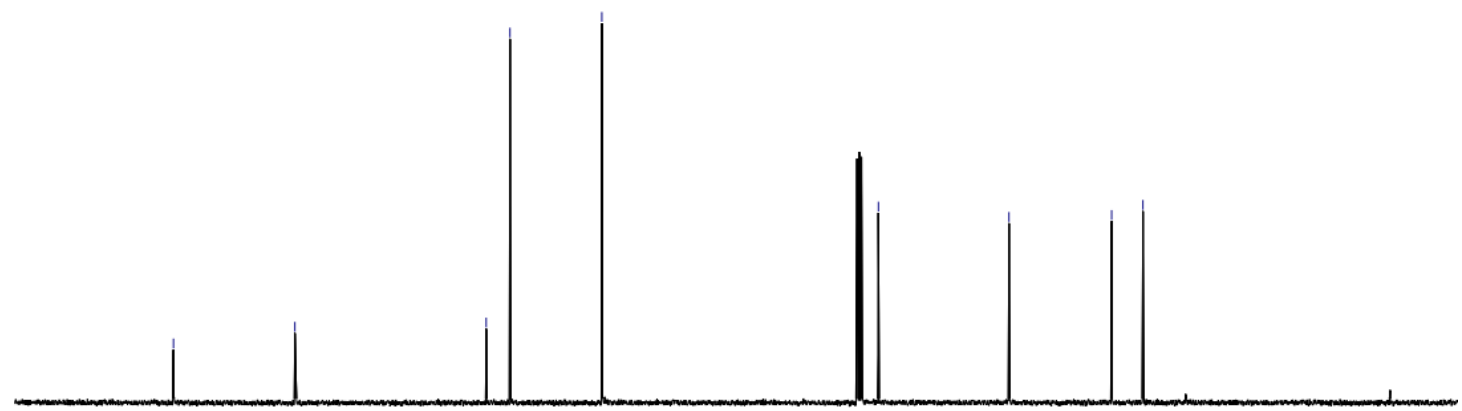

$\begin{array}{lllllllllllllllllll}190 & 180 & 170 & 160 & 150 & 140 & 130 & 120 & 110 & \underset{\mathrm{f} 1(\mathrm{ppm})}{90} & 80 & 70 & 60 & 50 & 40 & 30 & 20 & 10 & 0\end{array}$ 
<smiles>O=C1C[C@@H](c2ccc(C(F)(F)F)cc2)CO1</smiles>

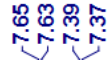<smiles>O=C1CC(c2ccc(C(F)(F)F)cc2)CO1</smiles>

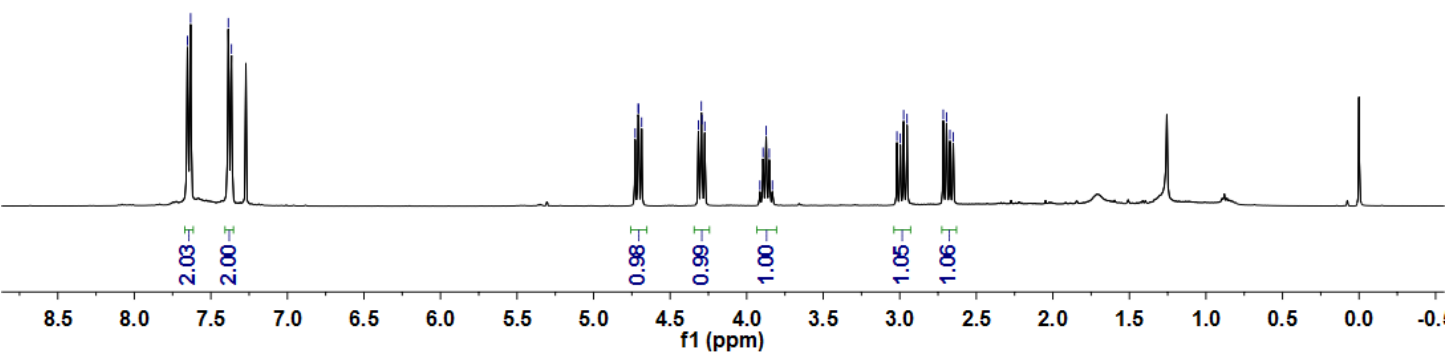

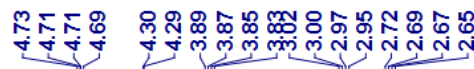

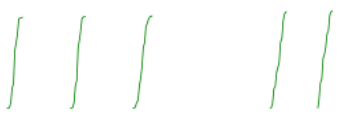

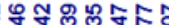

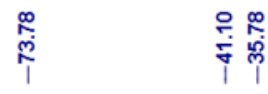
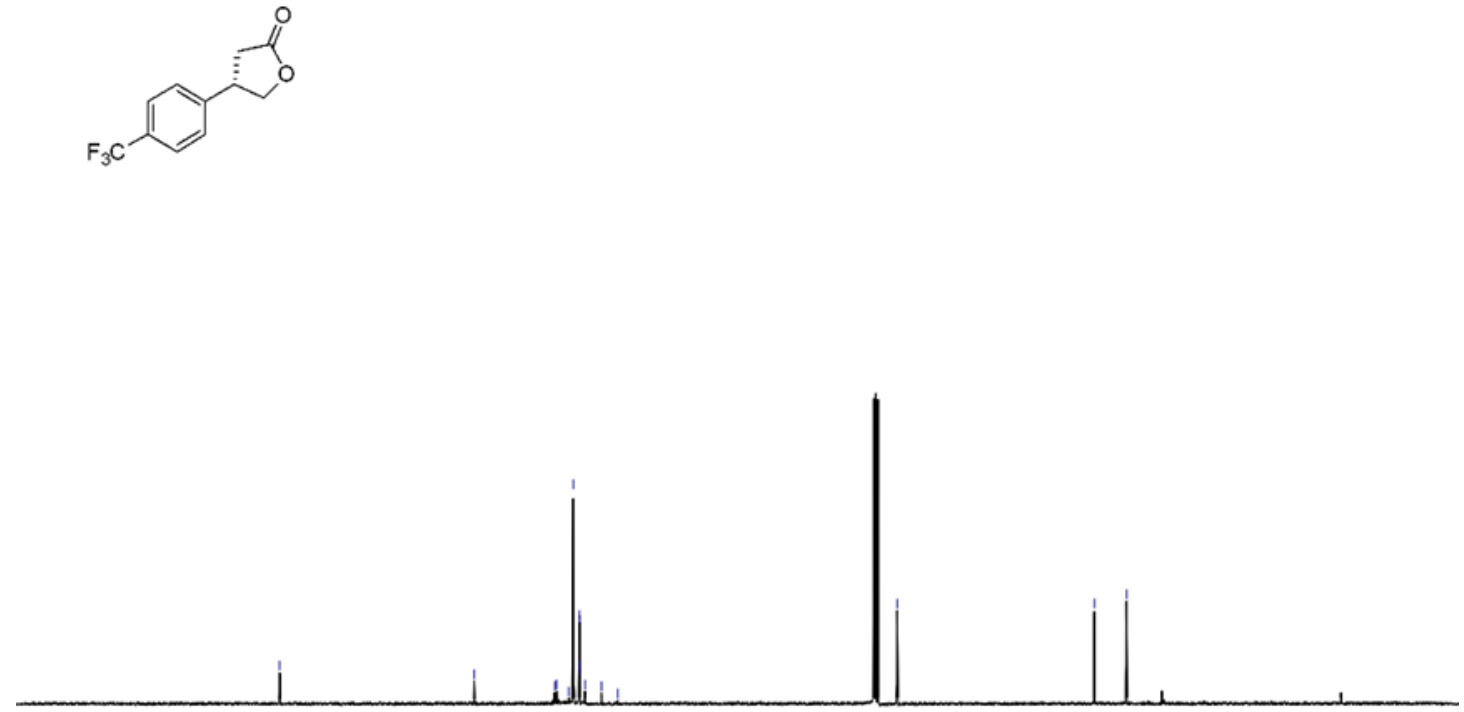

$\begin{array}{lllllllllllllllllllllll}210 & 200 & 190 & 180 & 170 & 160 & 150 & 140 & 130 & 120 & 110 \begin{array}{l}100 \\ \mathrm{f} 1(\mathrm{ppm})\end{array} & 90 & 80 & 70 & 60 & 50 & 40 & 30 & 20 & 10 & 0 & -10\end{array}$ 


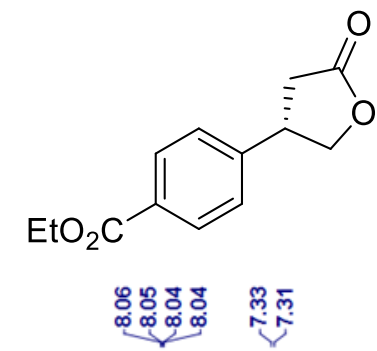

2h

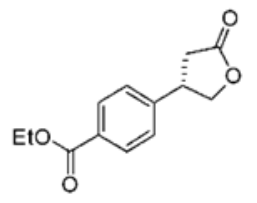

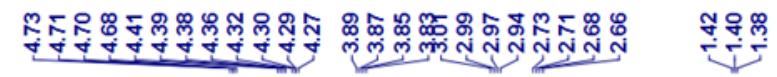

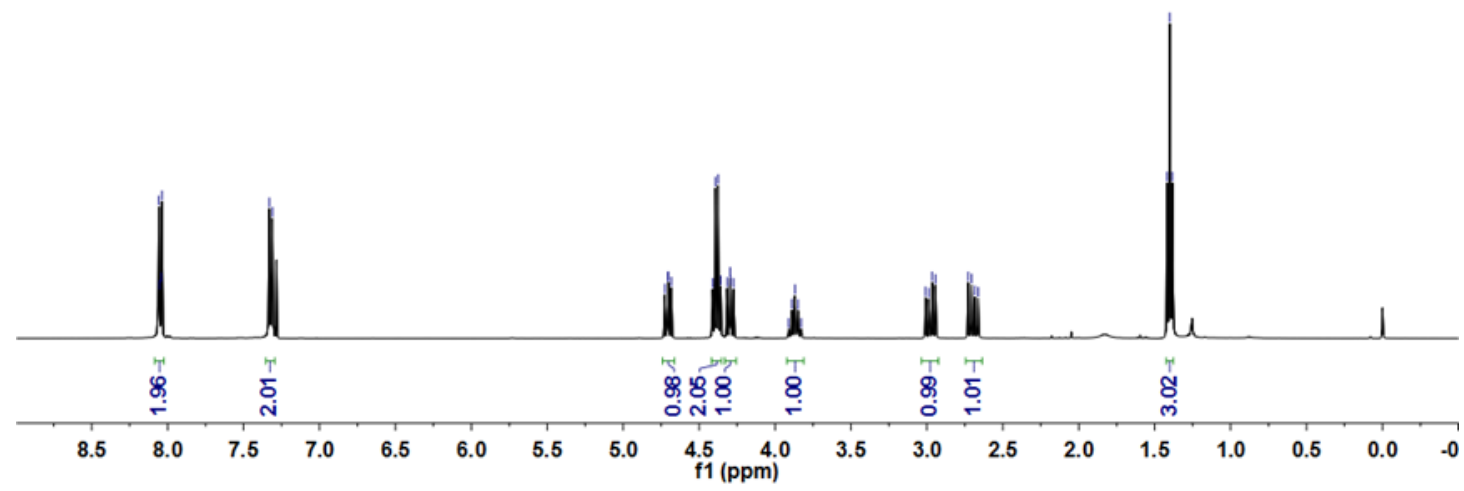

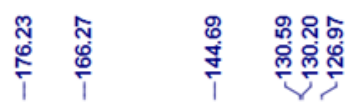

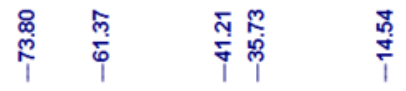

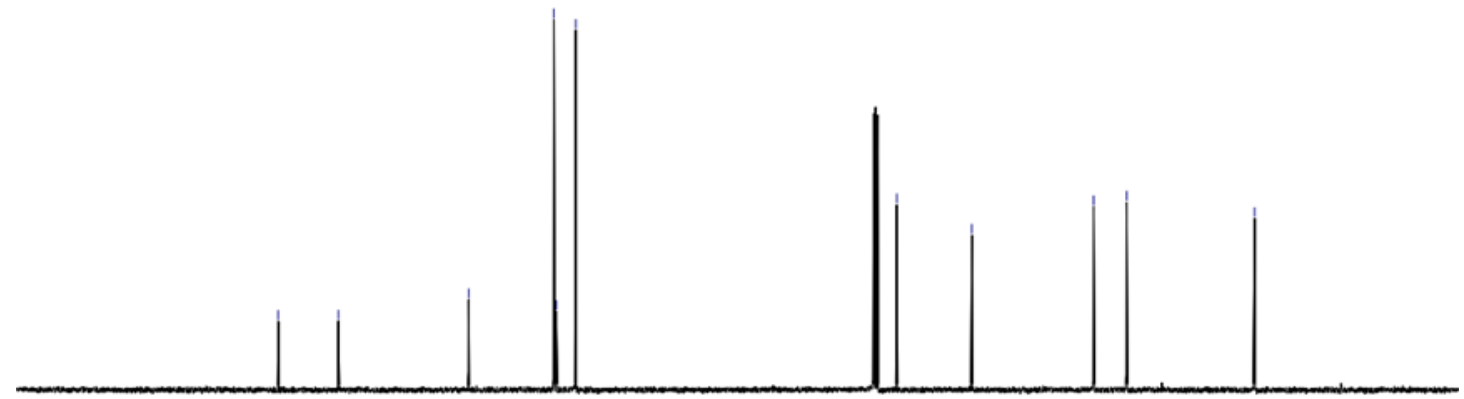

$\begin{array}{lllllllllllllllllllllll}210 & 200 & 190 & 180 & 170 & 160 & 150 & 140 & 130 & 120 & 110 \begin{array}{l}100 \\ \mathrm{f} 1(\mathrm{ppm})\end{array} & 90 & 80 & 70 & 60 & 50 & 40 & 30 & 20 & 10 & 0 & -10\end{array}$ 
<smiles>O=C1C[C@H](c2ccc(F)cc2)CO1</smiles>

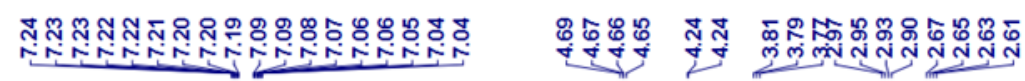
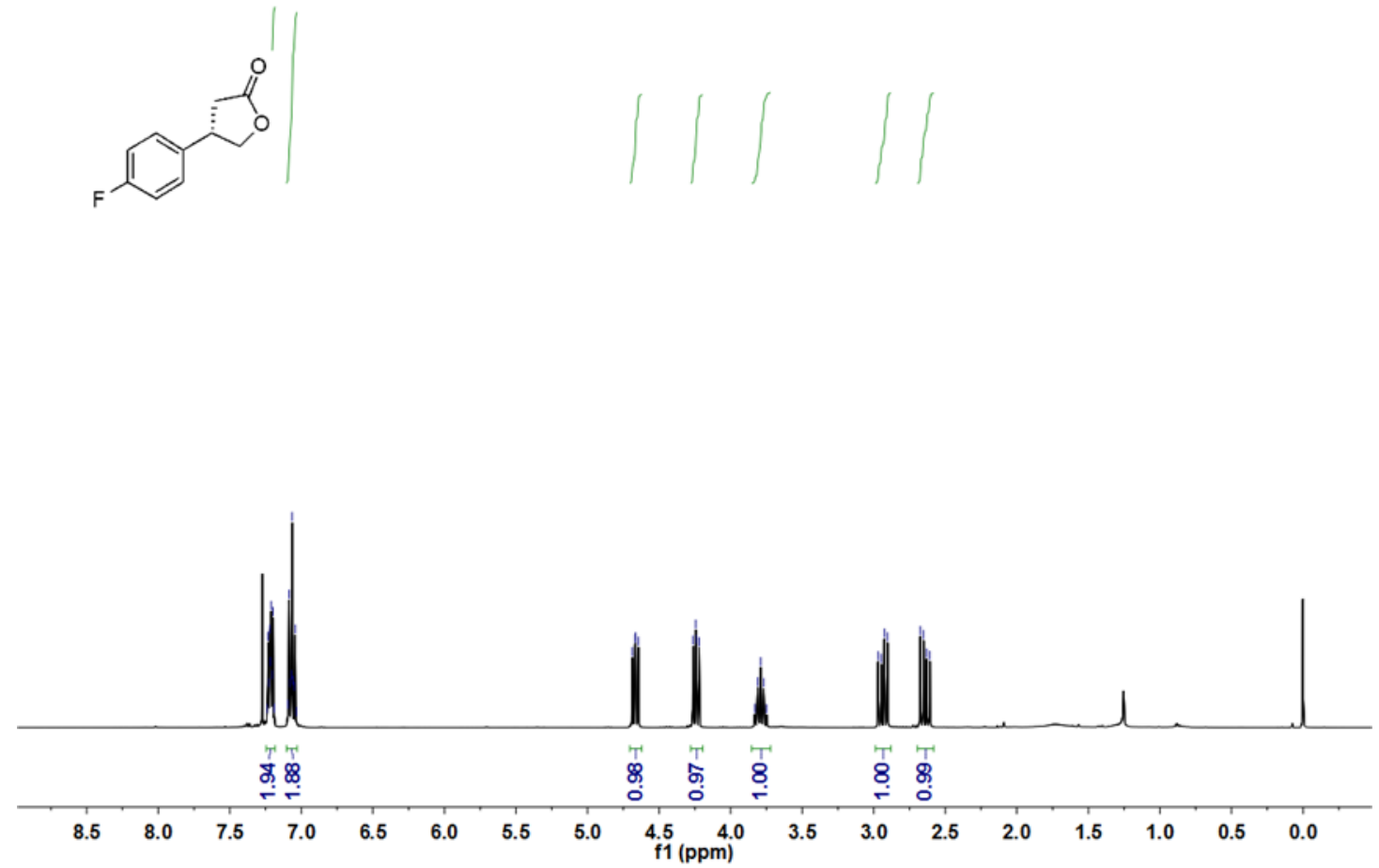

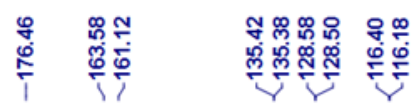

กิ
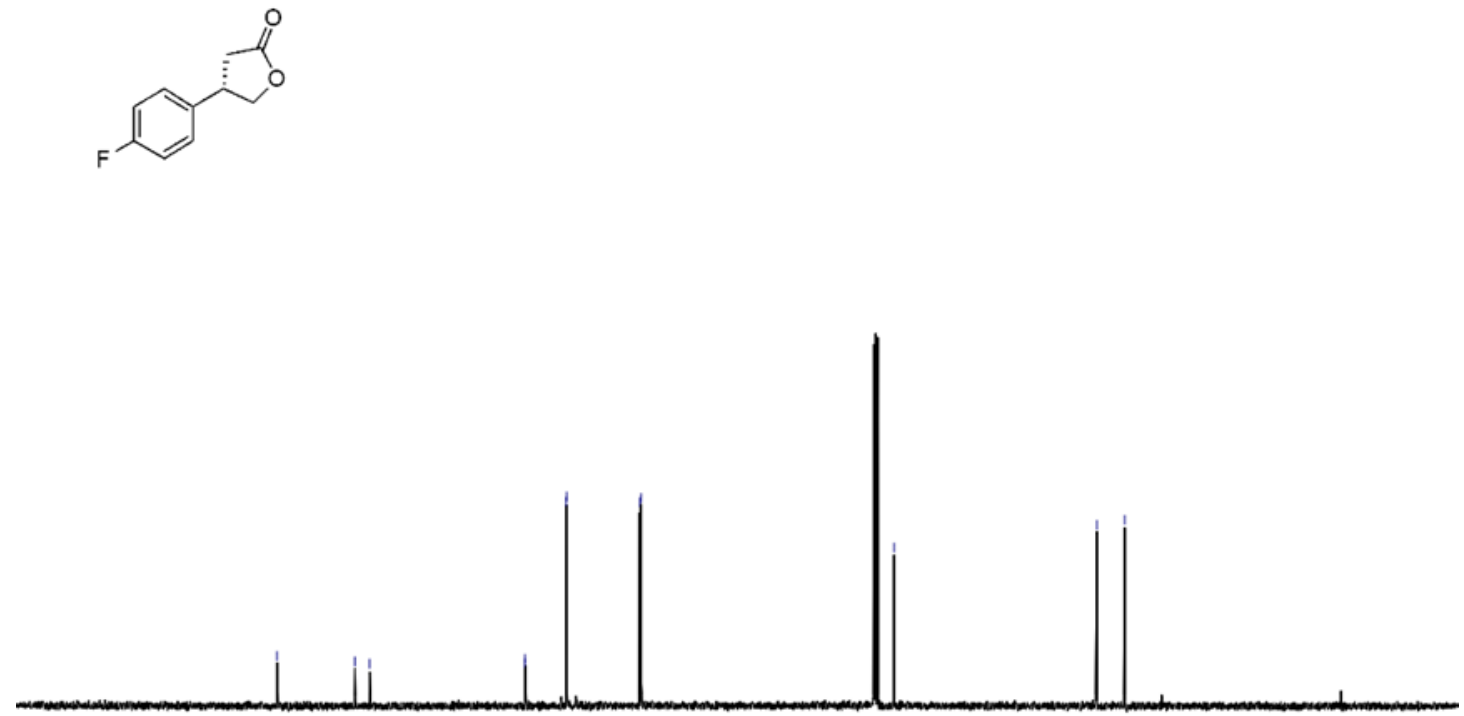

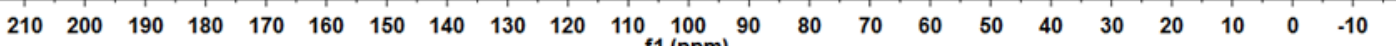


<smiles>O=C1C[C@H](c2ccc(Cl)cc2)CO1</smiles>

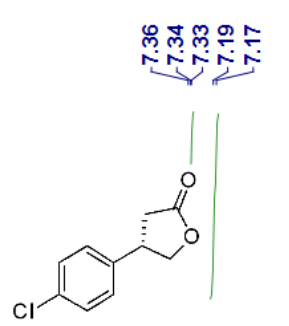

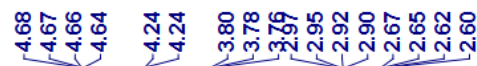

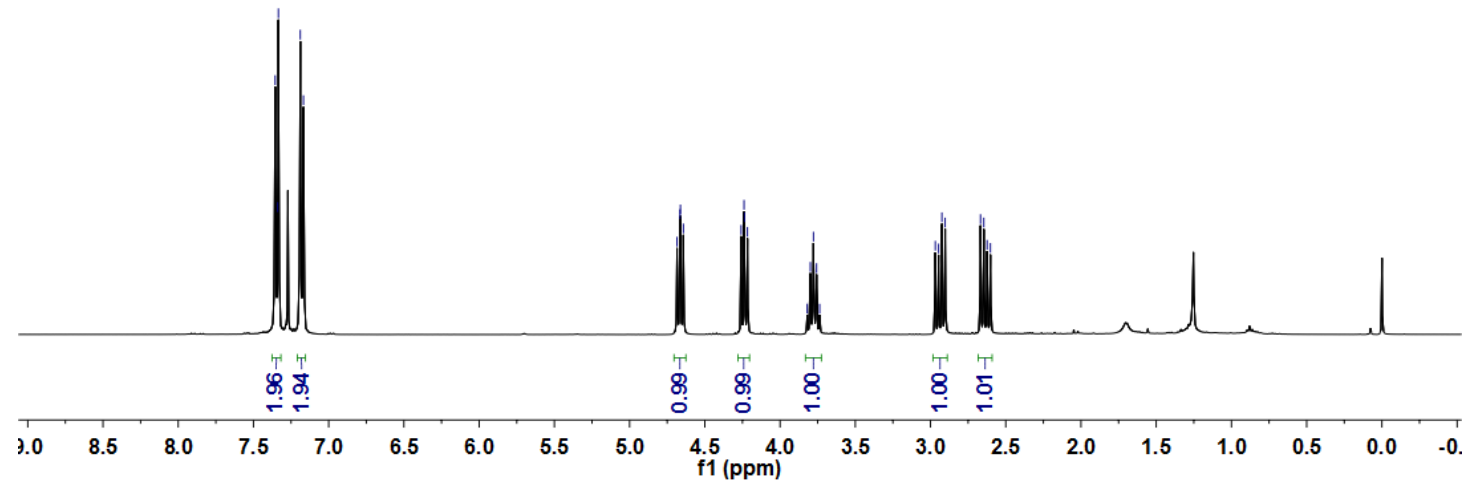

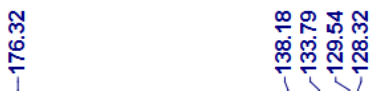

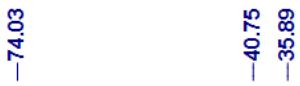

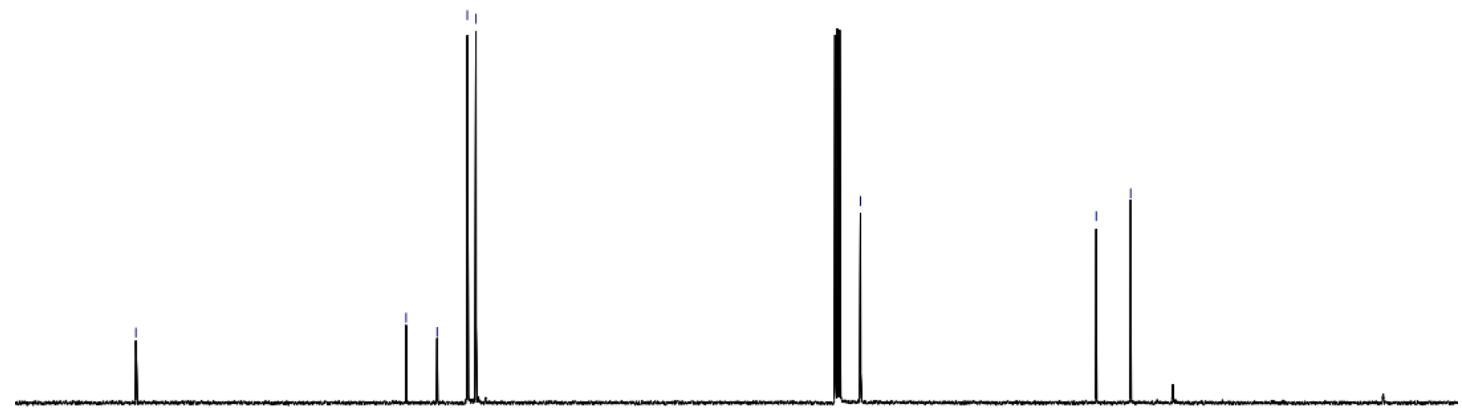

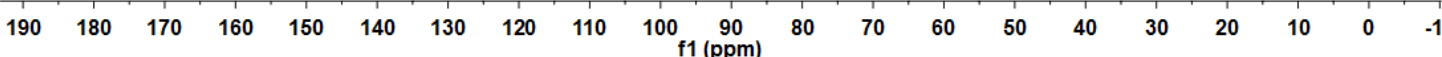


<smiles>O=C1C[C@H](c2cccc(F)c2)CO1</smiles>

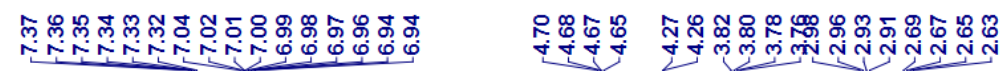
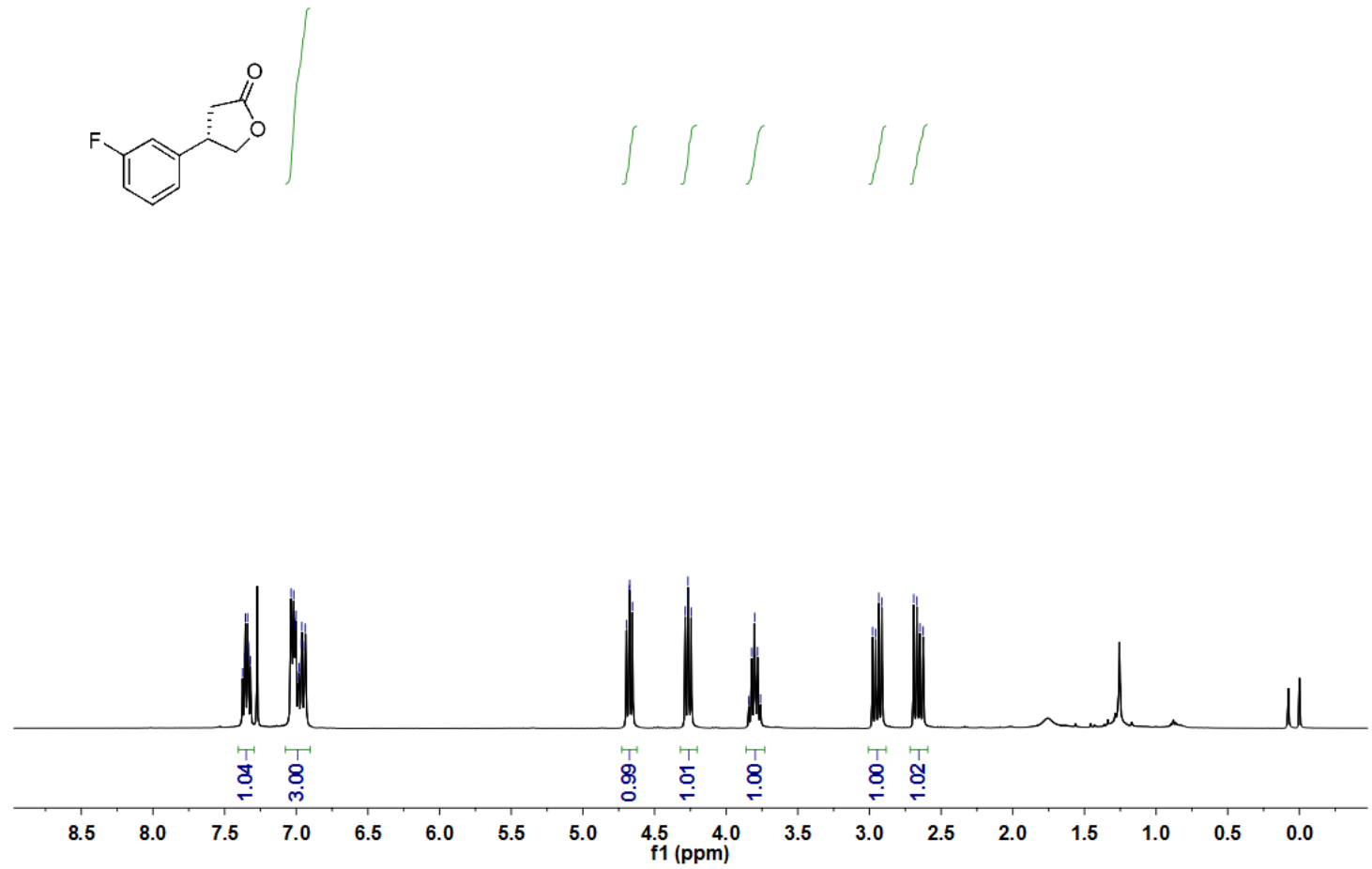

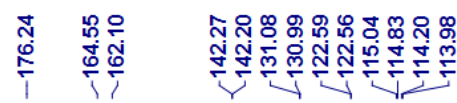
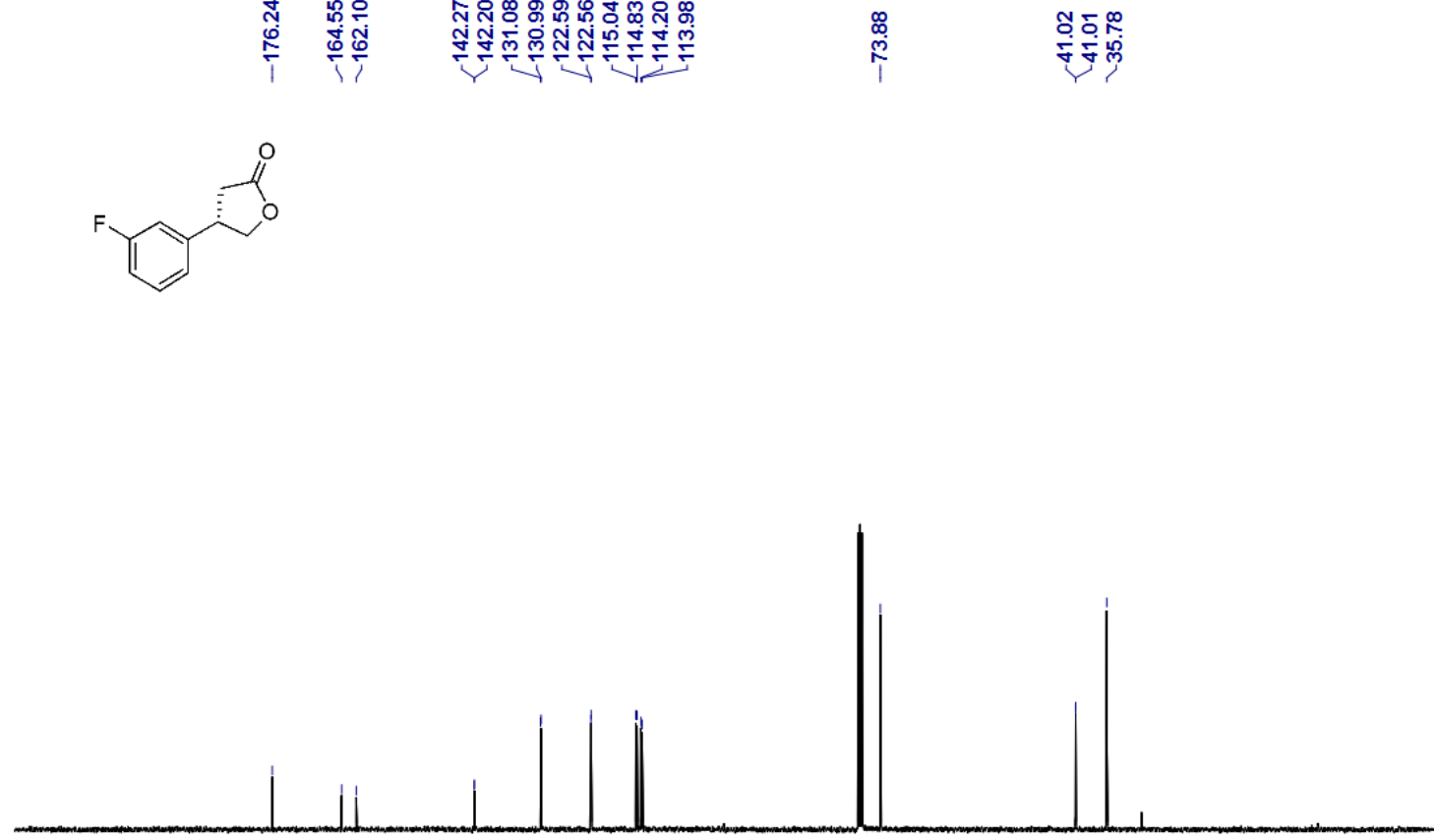

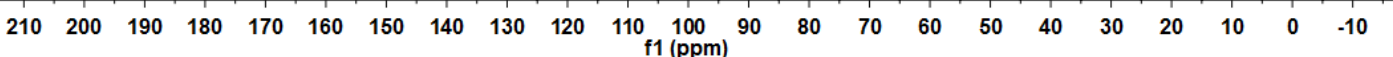


21

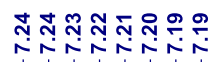

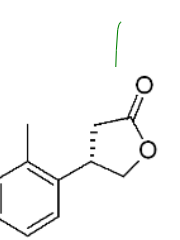

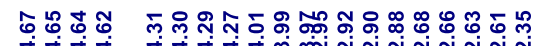

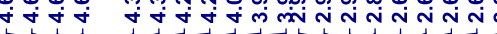

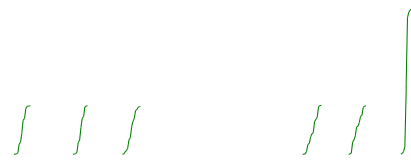

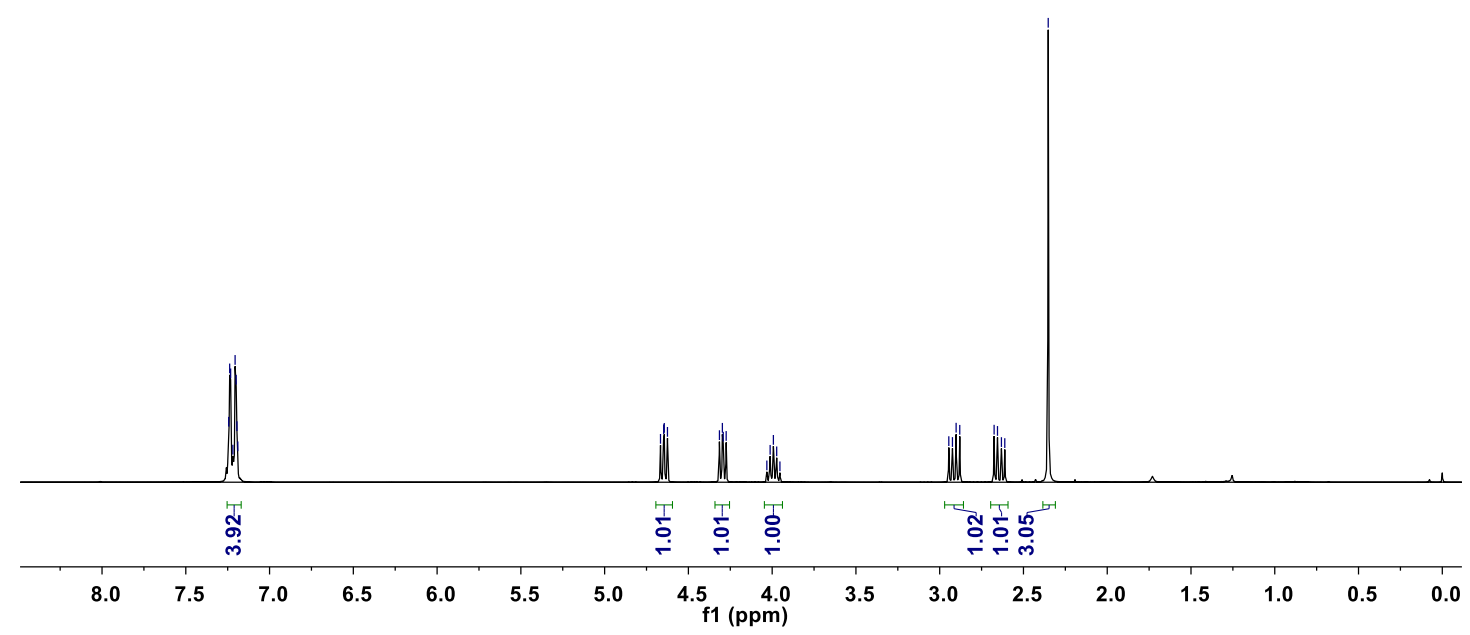

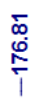
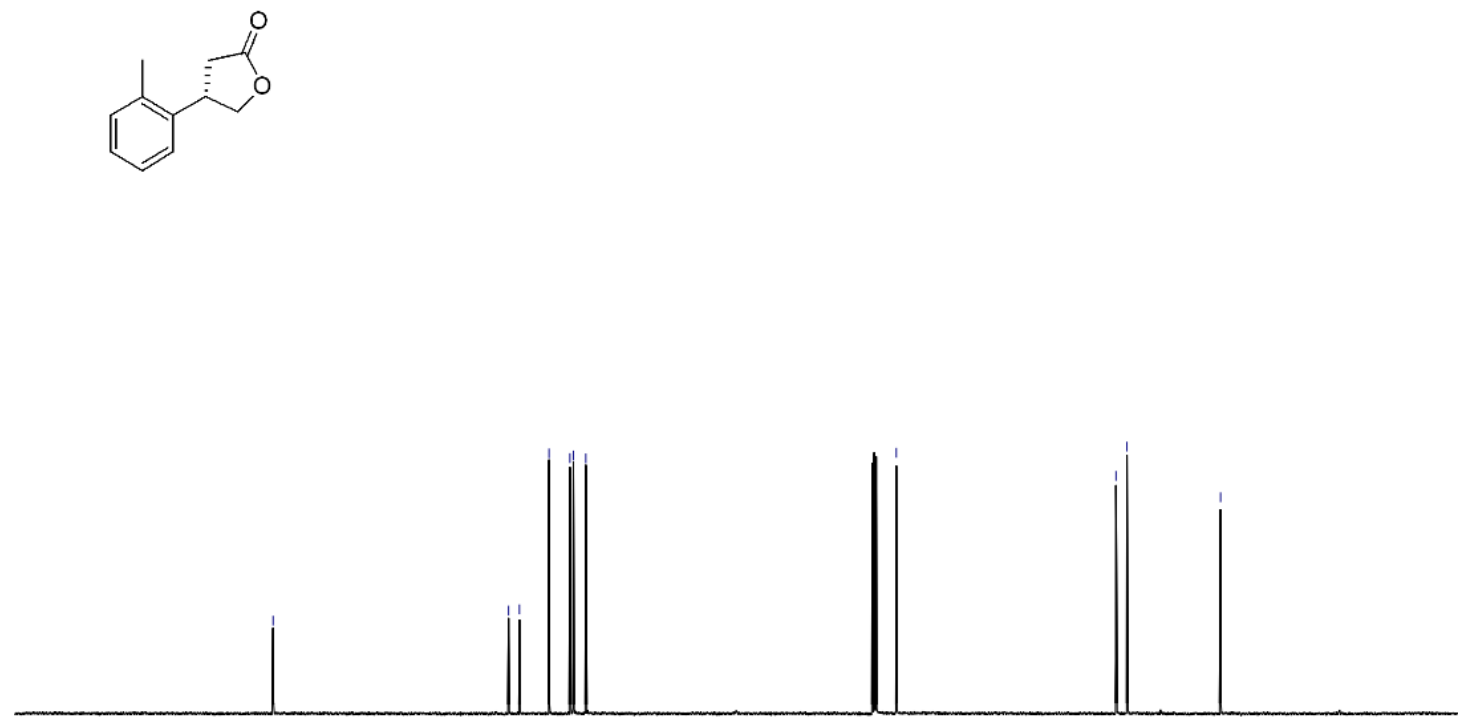

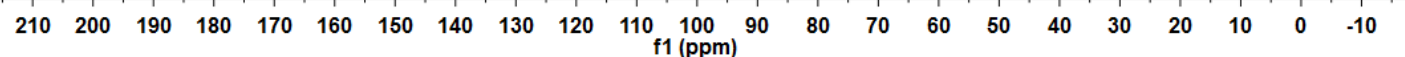


<smiles>Cc1ccc([C@H]2COC(=O)C2)cc1C</smiles>

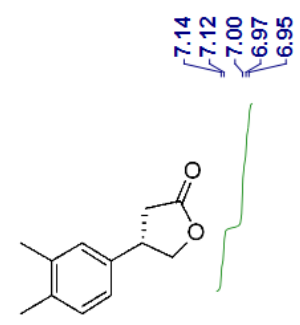

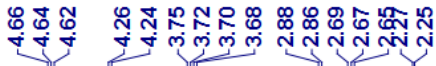

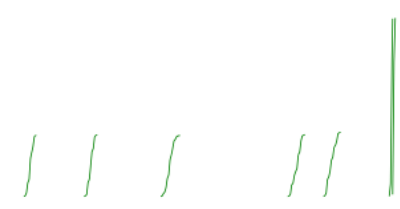

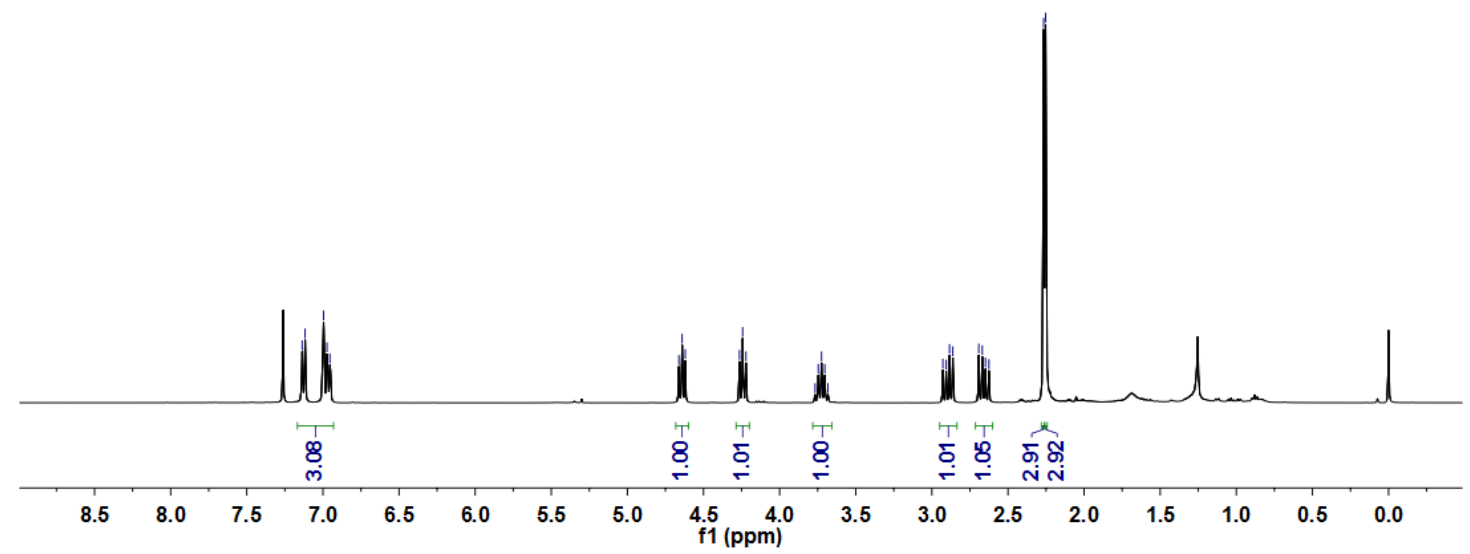

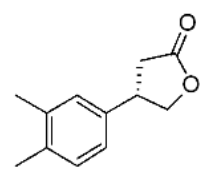

密

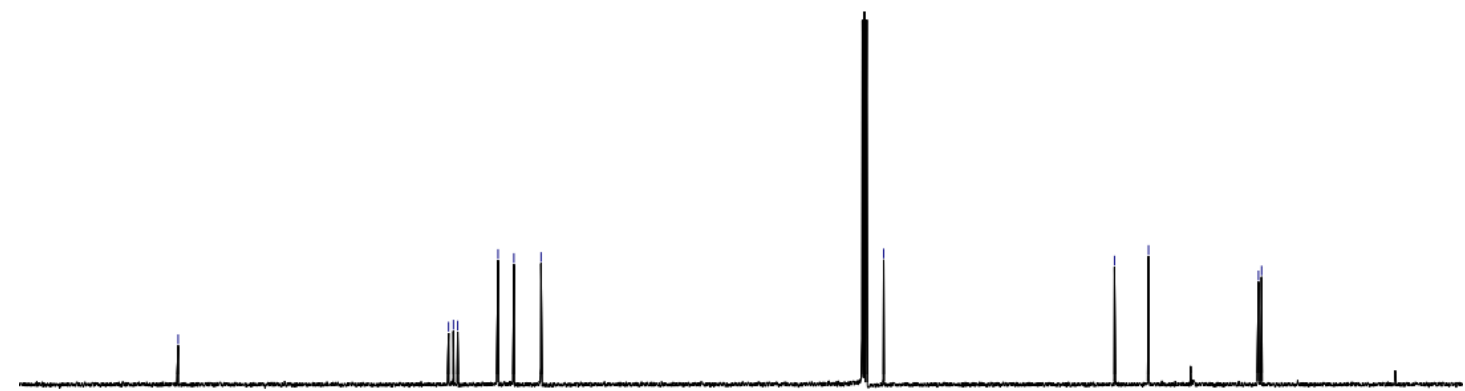

$\begin{array}{lllllllllll}30 & 190 & 180 & 170 & 160 & 150 & 140 & 130 & 120 & 110 & 100 \\ 90\end{array}$ 
<smiles>COc1ccc([C@H]2COC(=O)C2)cc1OC</smiles>
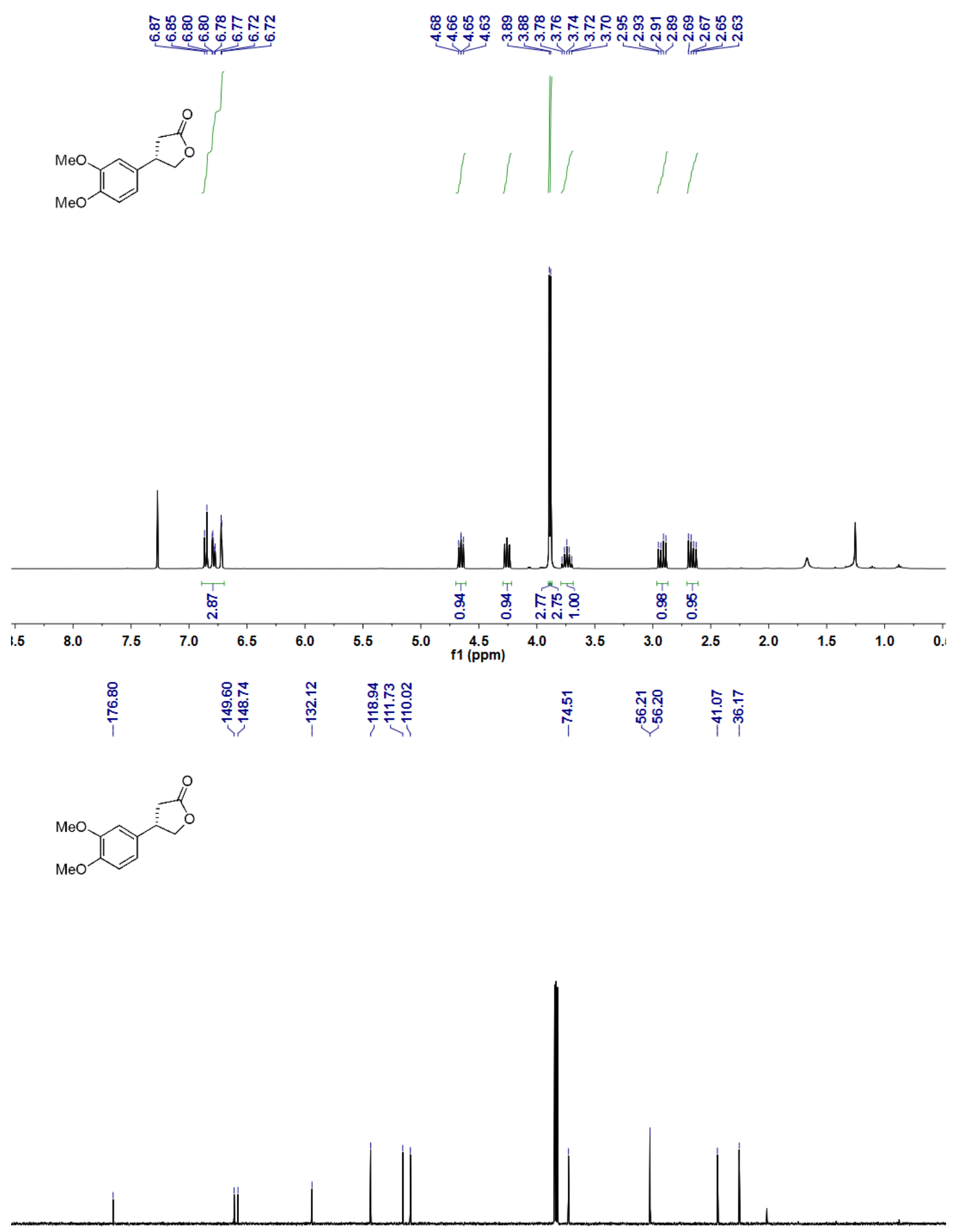

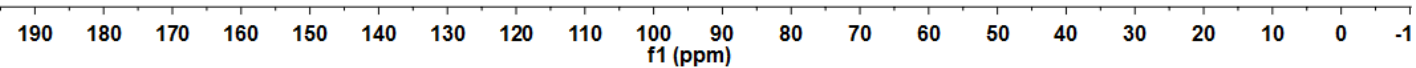


<smiles>O=C1C[C@H](c2ccc3c(c2)OCCO3)CO1</smiles>

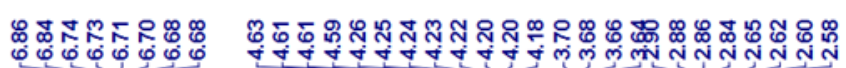
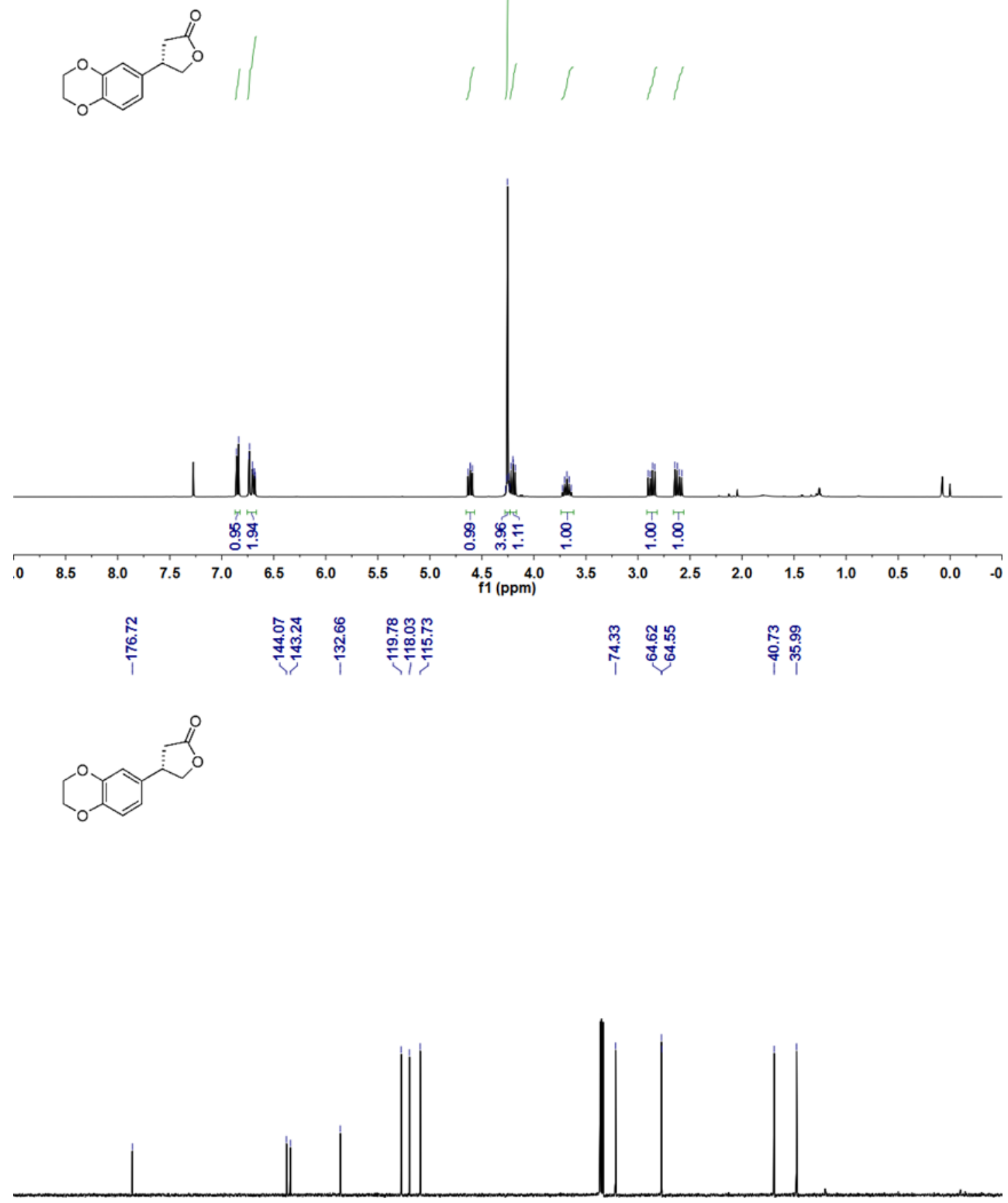

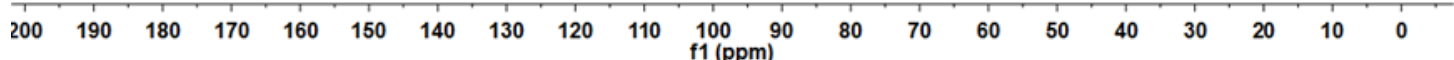


$\underbrace{1}_{2 p}$

悹品<smiles>Cc1cc(C)cc(C2COC(=O)C2)c1</smiles>

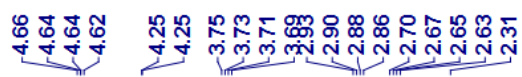

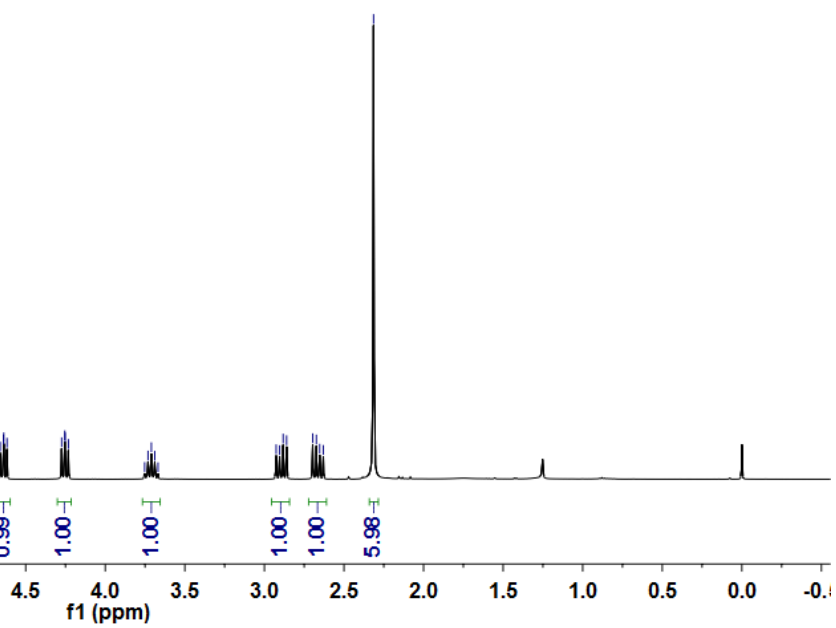

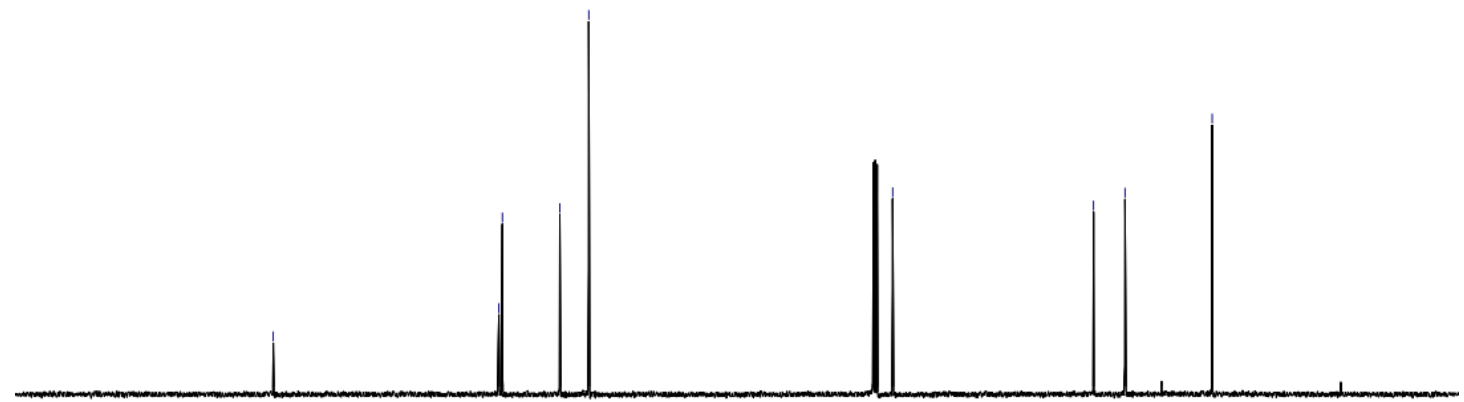

$\begin{array}{lllllllllllllllllllllll}210 & 200 & 190 & 180 & 170 & 160 & 150 & 140 & 130 & 120 & 110 & 100 & 90 & 80 & 70 & 60 & 50 & 40 & 30 & 20 & 10 & 0 & -10\end{array}$ 
<smiles>O=C1C[C@H](c2ccc3ccccc3c2)CO1</smiles>

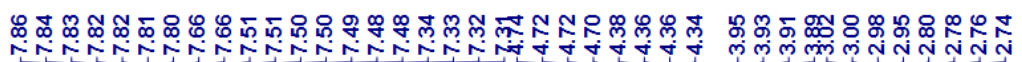
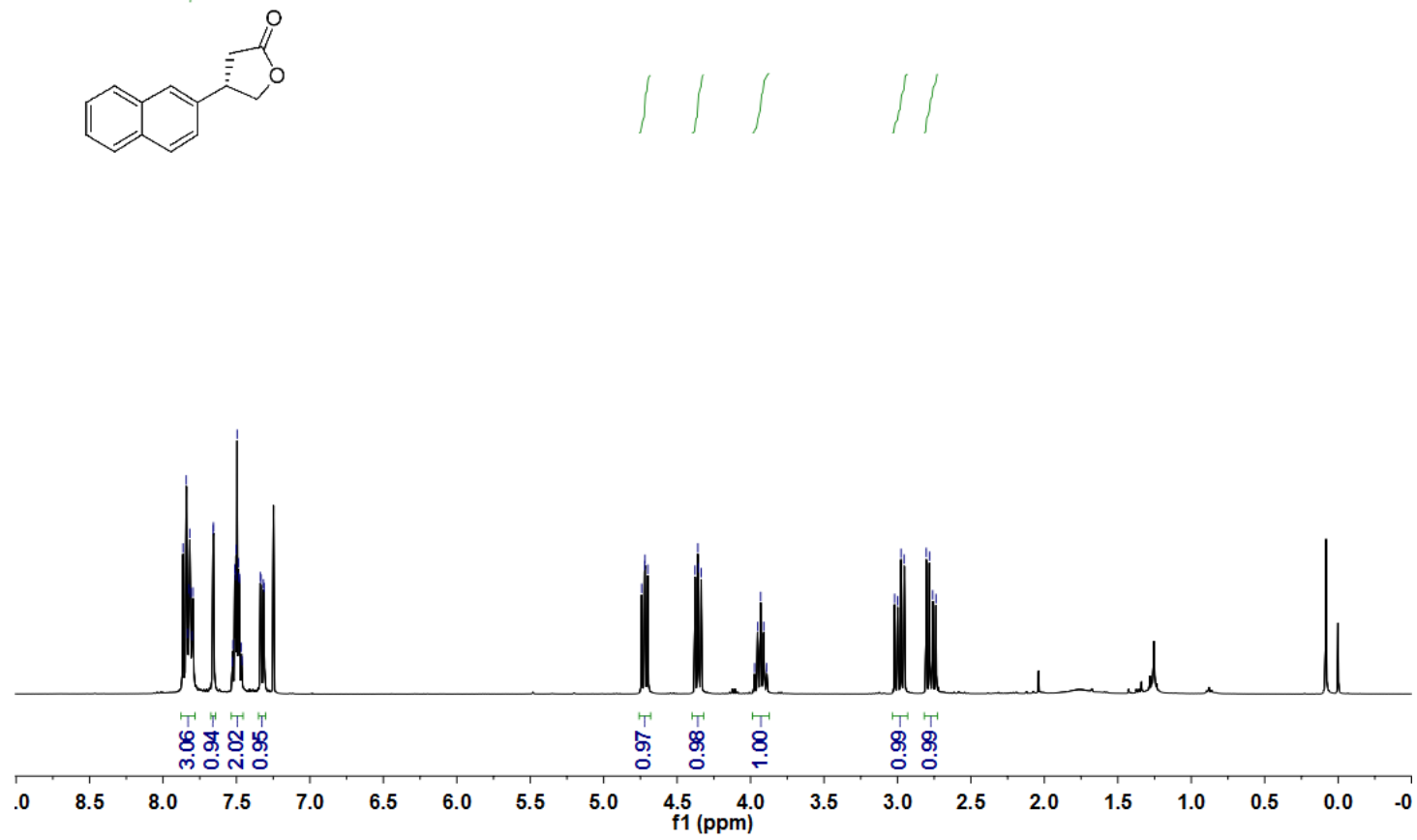

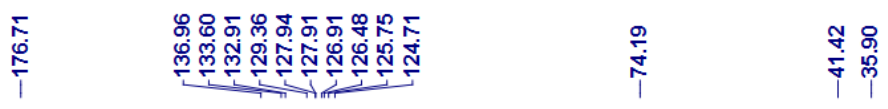
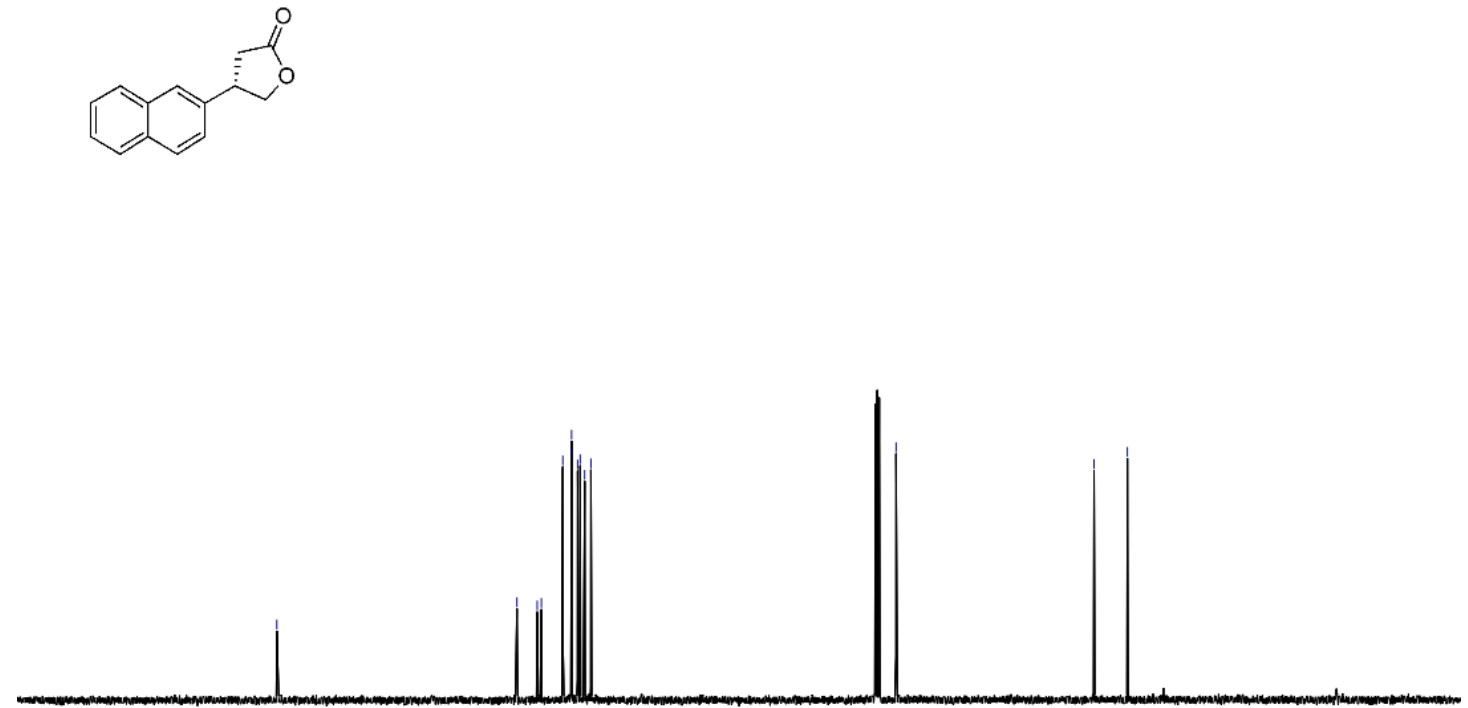

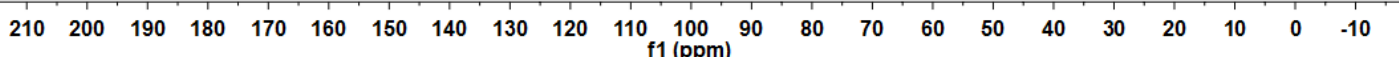


<smiles>O=C1C[C@H](c2ccco2)CO1</smiles>

$\underbrace{\infty 00}$
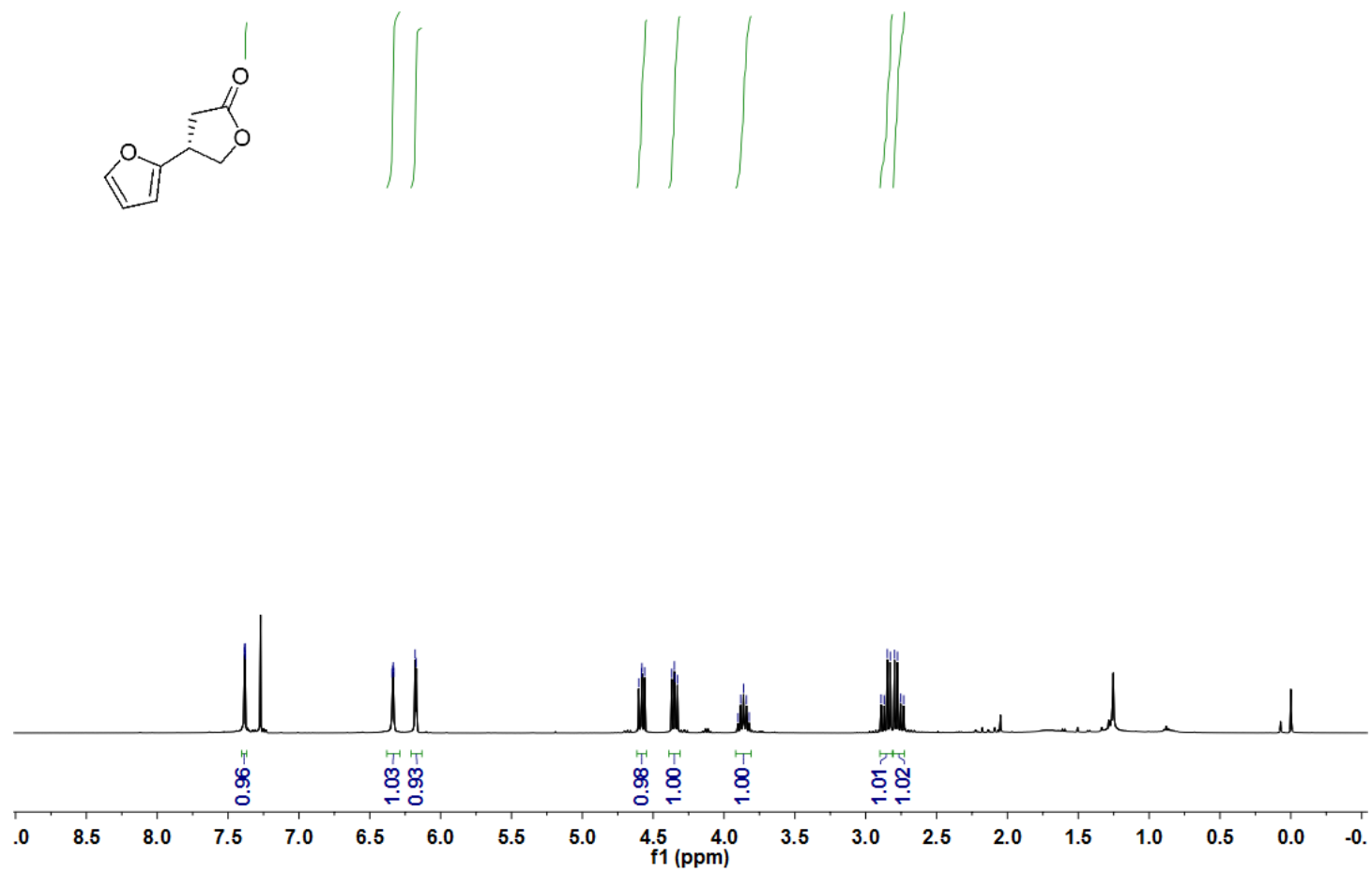

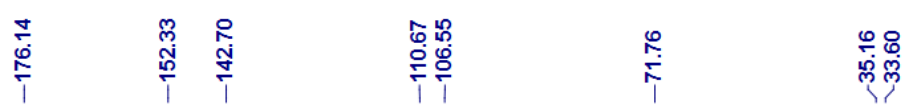
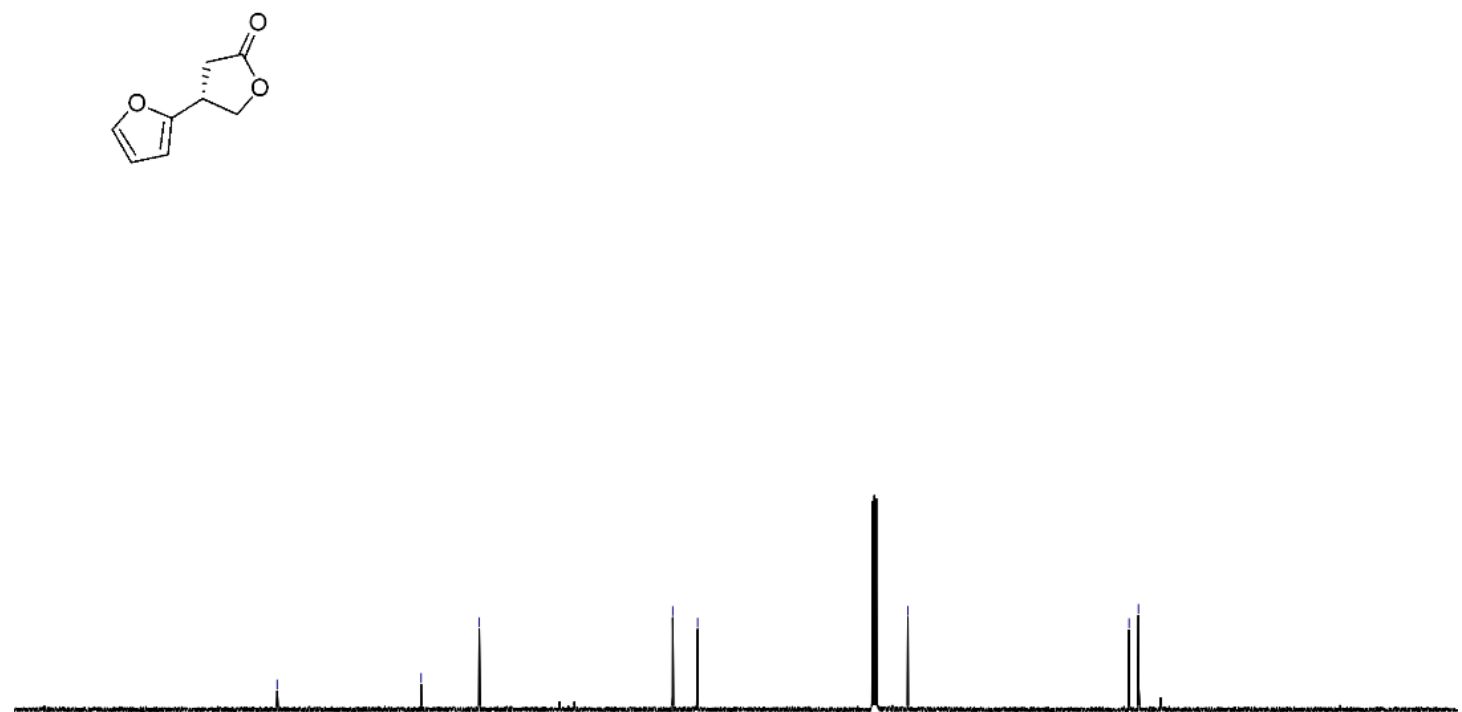

$\begin{array}{lllllllllllllllllllllll}210 & 200 & 190 & 180 & 170 & 160 & 150 & 140 & 130 & 120 & 110 \underset{\mathrm{f} 1(\mathrm{ppm})}{100} & 90 & 80 & 70 & 60 & 50 & 40 & 30 & 20 & 10 & 0 & -10\end{array}$ 
<smiles>O=C1C[C@H](c2ccc3occc3c2)CO1</smiles>

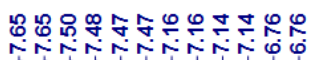

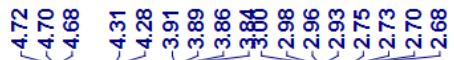
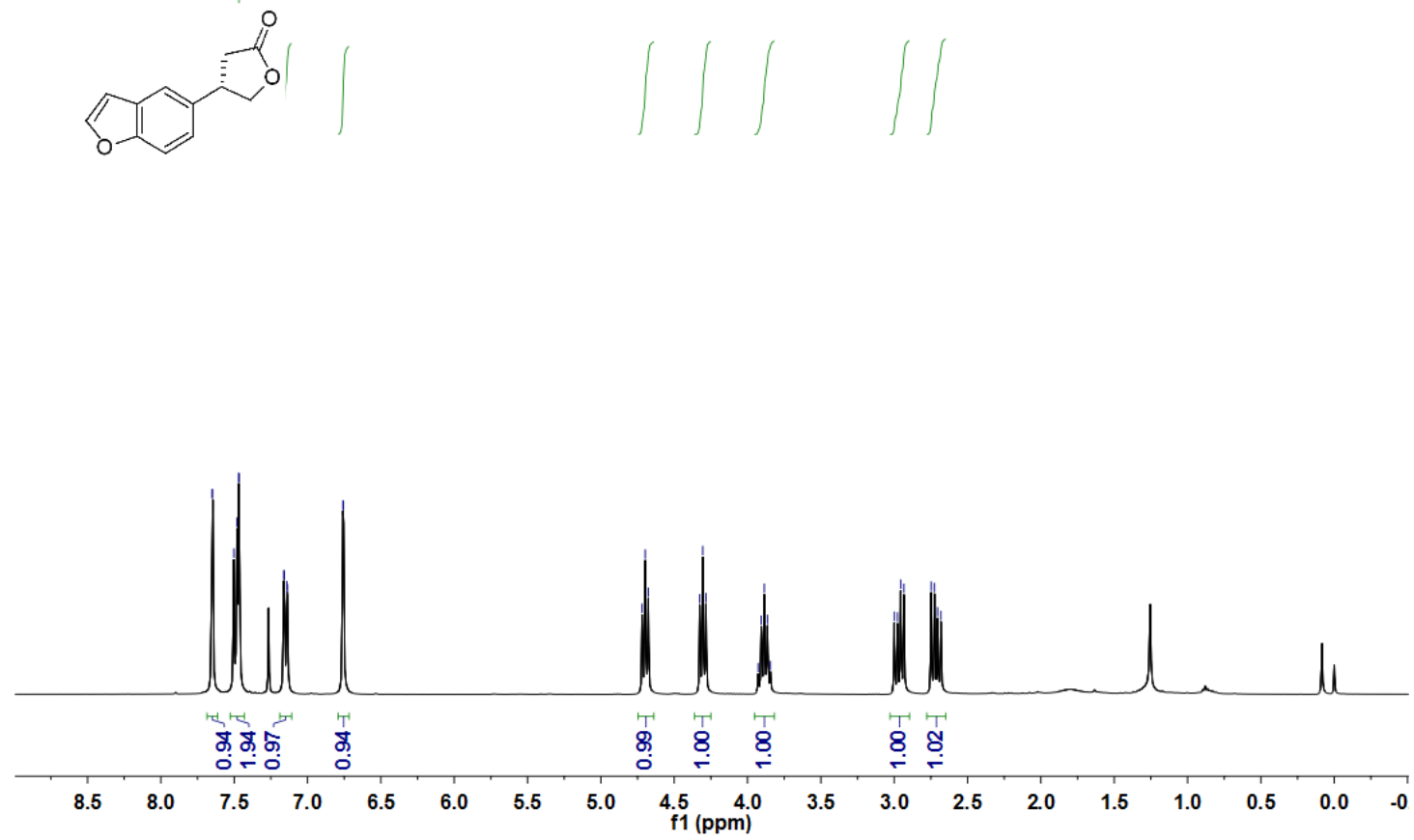

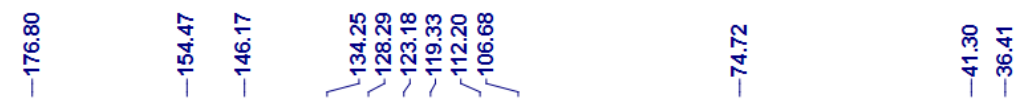
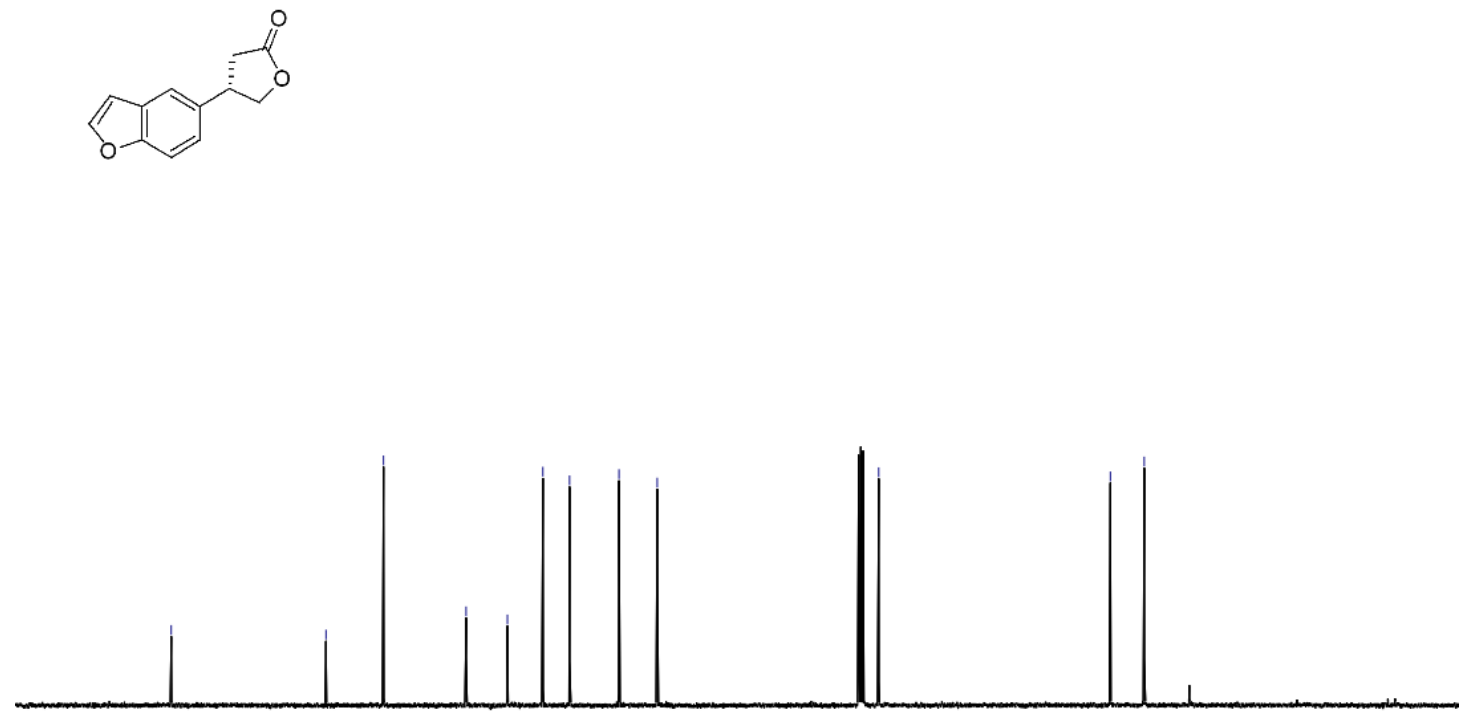

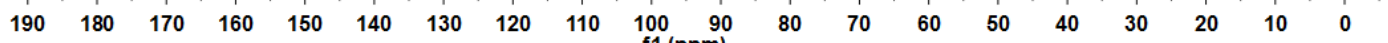


<smiles>[Z1]C[C@H]1COC(=O)C1</smiles>

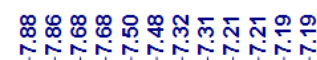

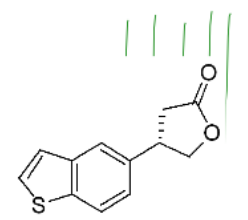

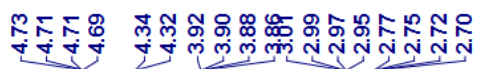

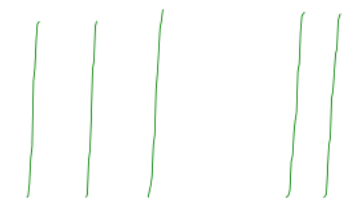

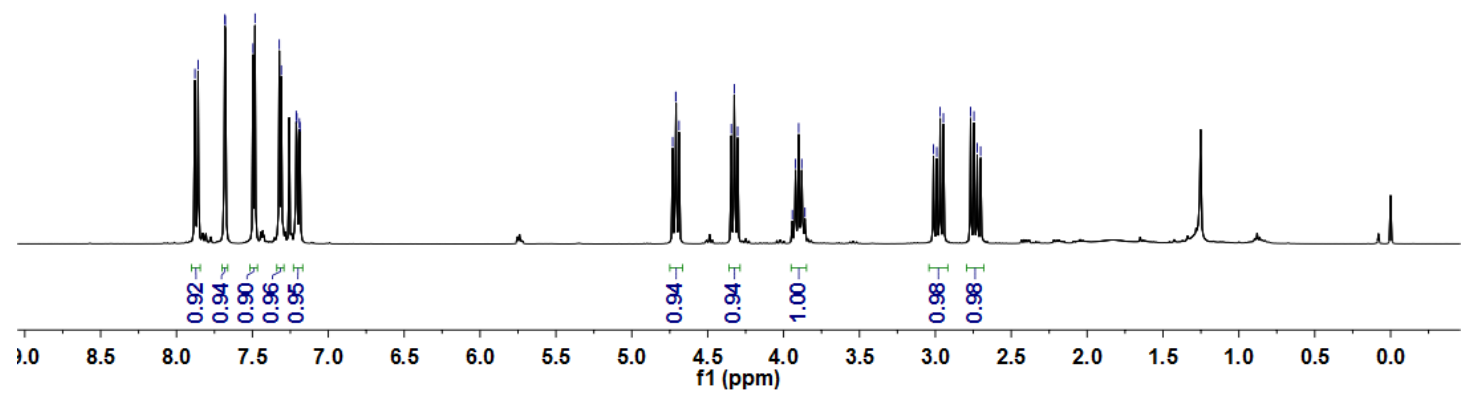

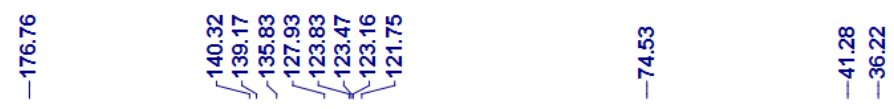
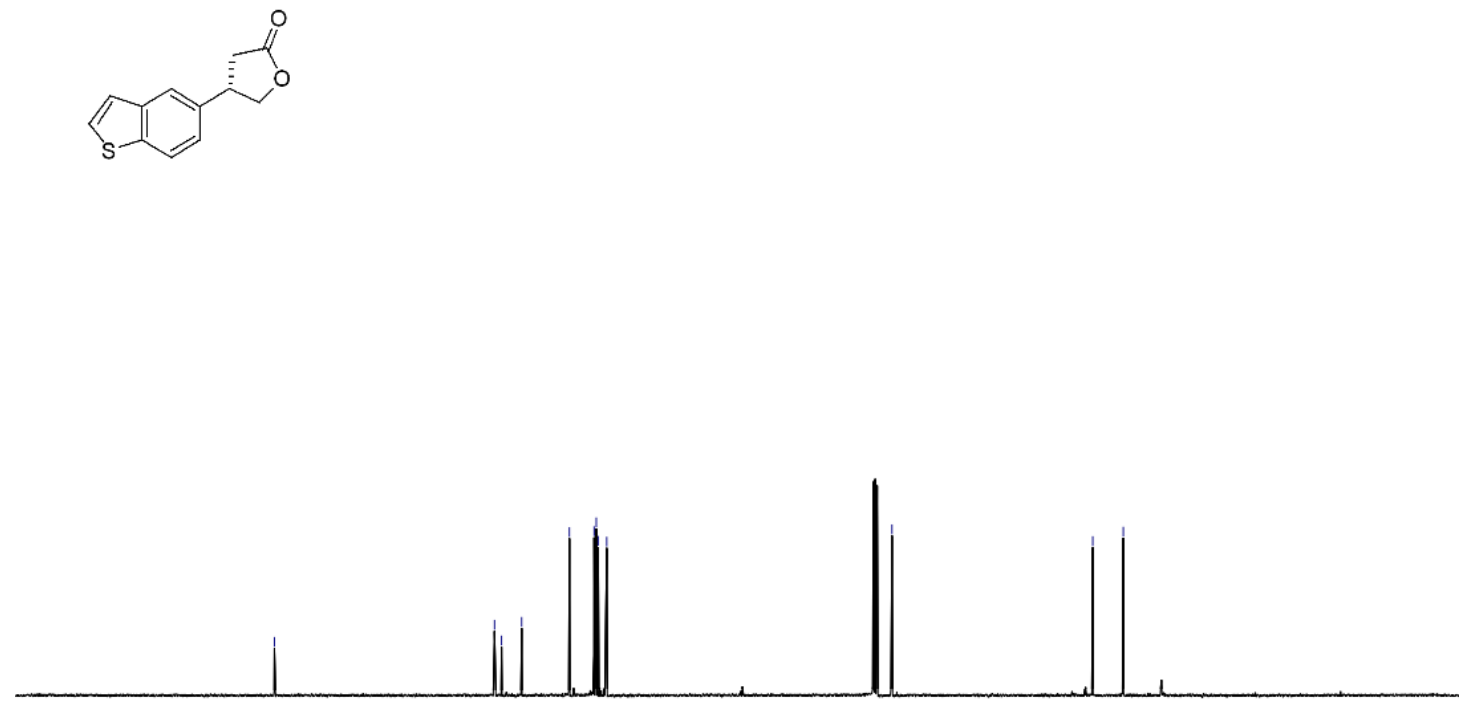

$\begin{array}{llllllllllllllllllllll}210 & 200 & 190 & 180 & 170 & 160 & 150 & 140 & 130 & 120 & 110 \begin{array}{l}100 \\ \mathrm{f} 1(\mathrm{ppm})\end{array} & 90 & 80 & 70 & 60 & 50 & 40 & 30 & 20 & 10 & 0 & -10\end{array}$ 
<smiles>CC(C)(C)[14CH3]</smiles>

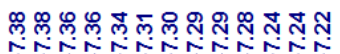

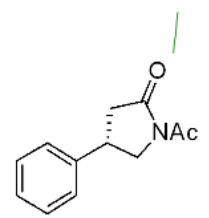

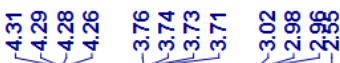

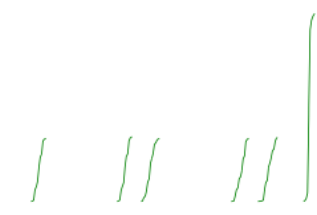

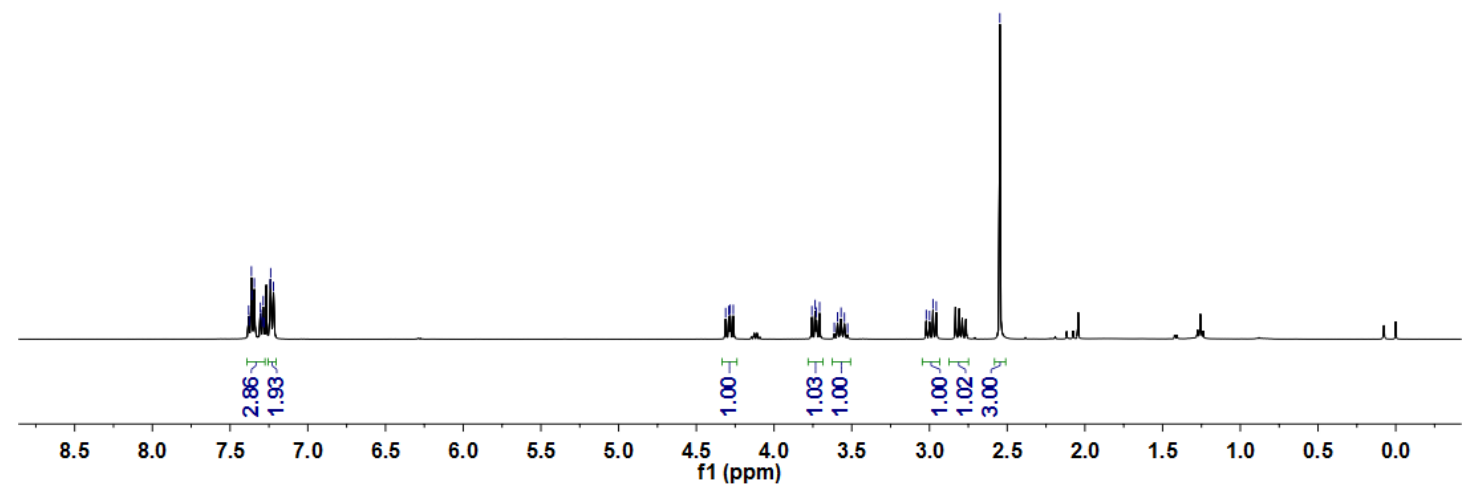

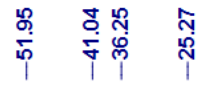

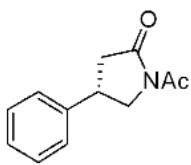

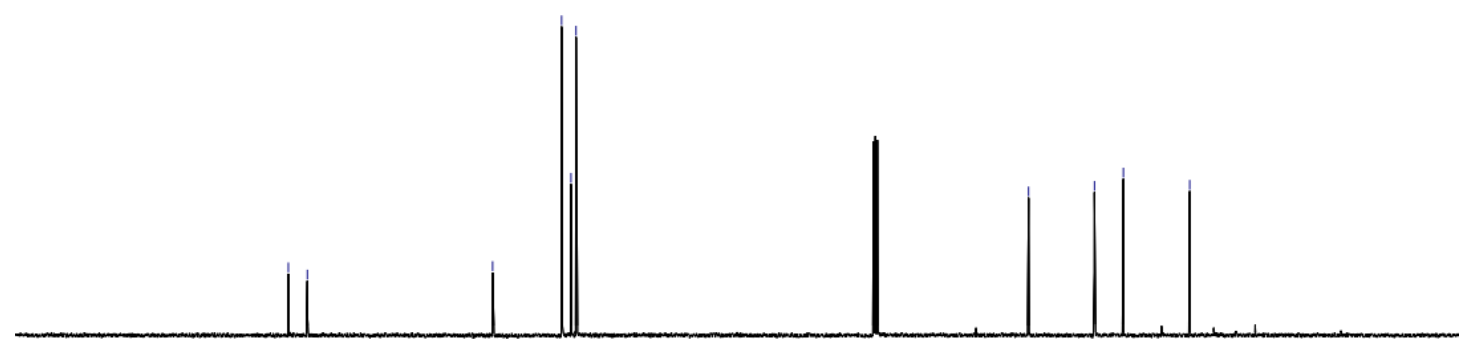

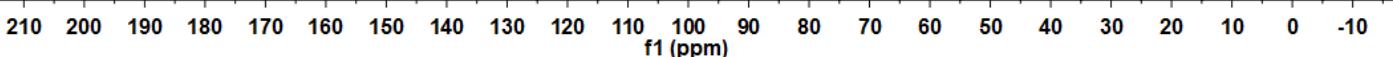


<smiles>Cc1ccc([C@@H]2CC(=O)N(C(C)(C)C)C2)cc1</smiles><smiles>Cc1ccc(CCNC(=O)C2CC2)cc1</smiles>

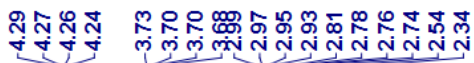
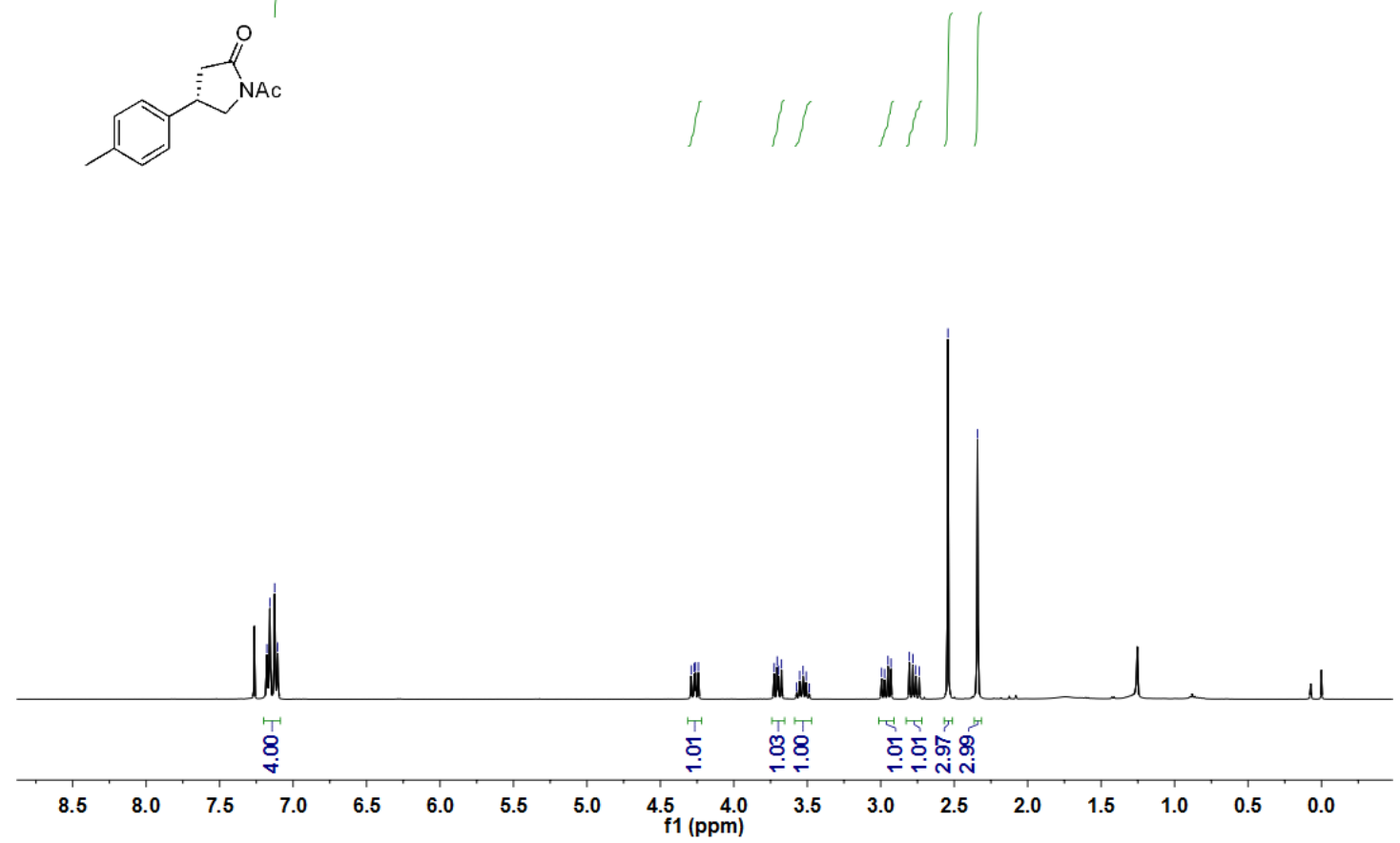

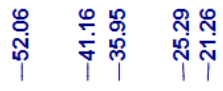
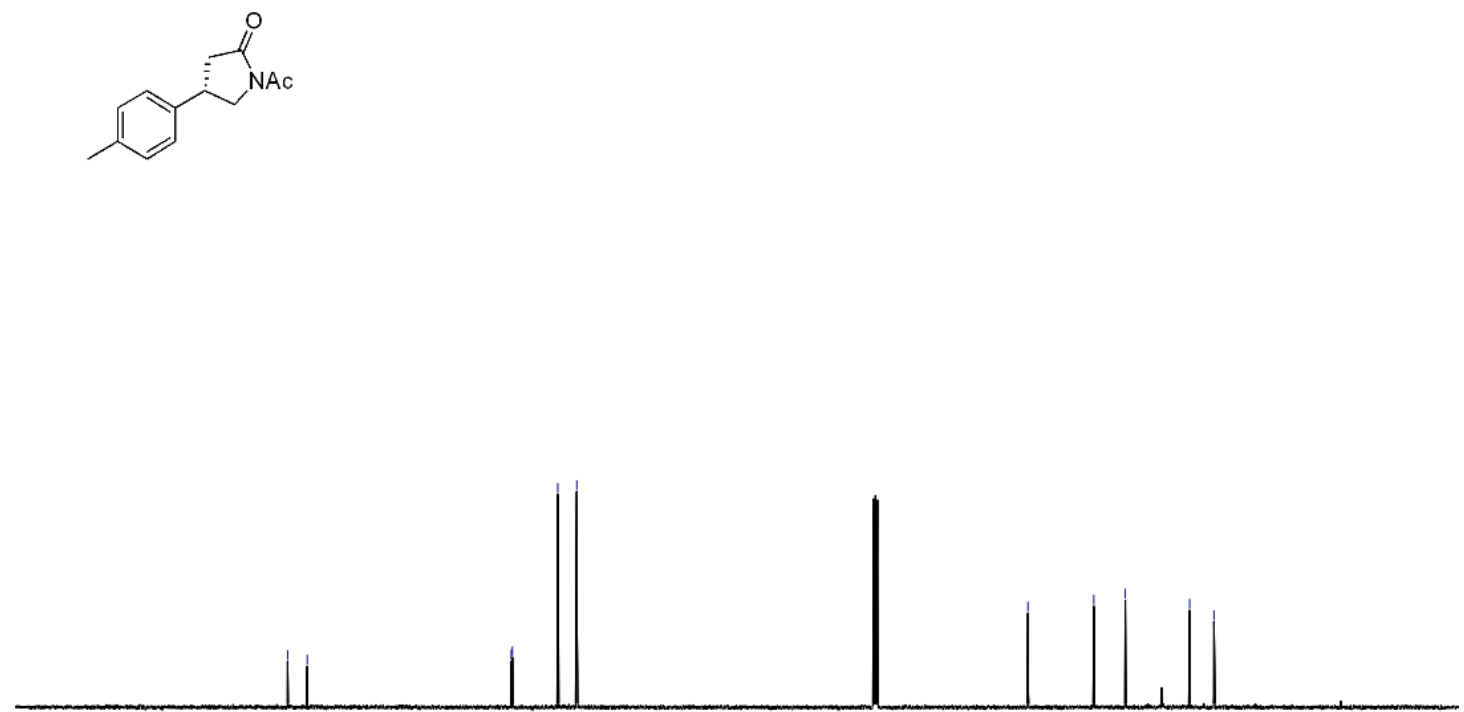

$\begin{array}{llllllllllllllllllllll}210 & 200 & 190 & 180 & 170 & 160 & 150 & 140 & 130 & 120 & 110 \begin{array}{l}100 \\ \mathrm{f} 1(\mathrm{ppm})\end{array} & 90 & 80 & 70 & 60 & 50 & 40 & 30 & 20 & 10 & 0 & -10\end{array}$ 

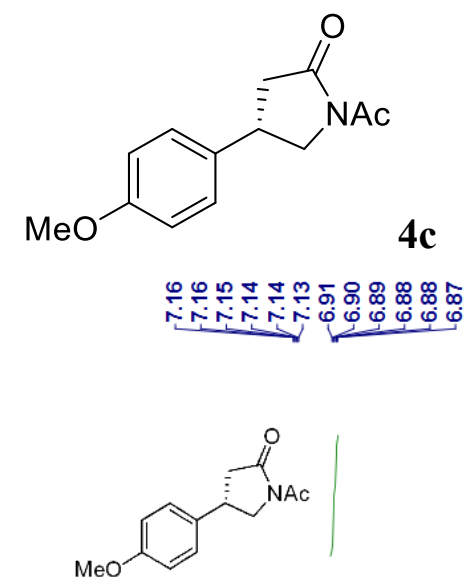

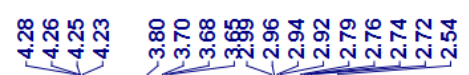

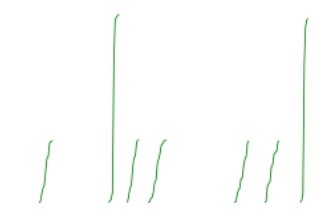

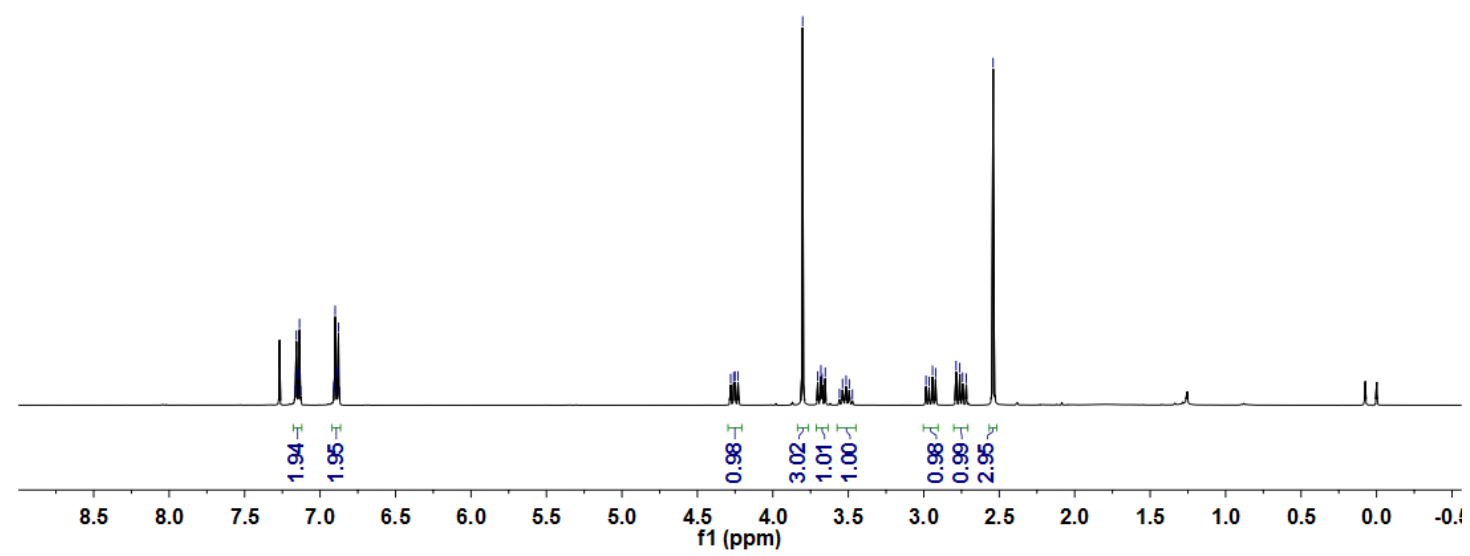

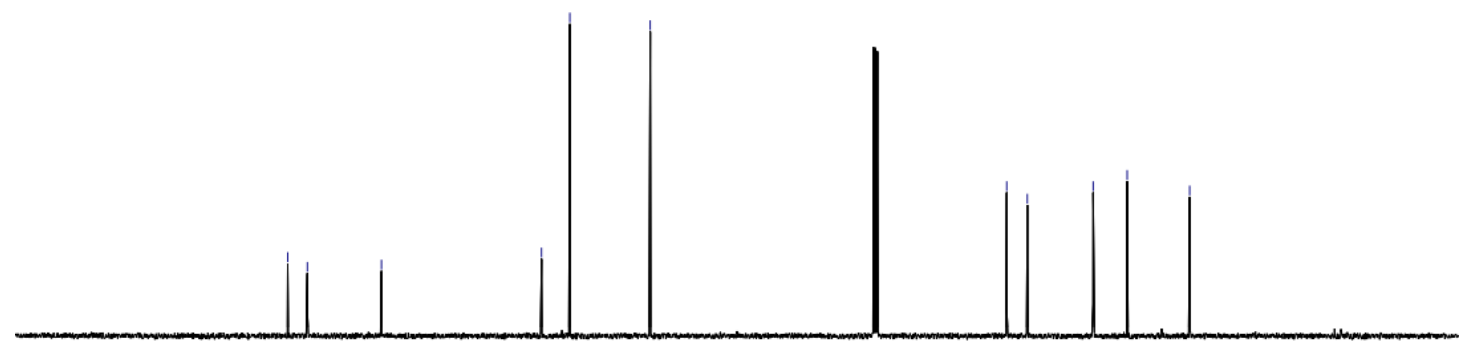

$\begin{array}{llllllllllllllllllllll}210 & 200 & 190 & 180 & 170 & 160 & 150 & 140 & 130 & 120 & 110 \begin{array}{l}100 \\ \mathrm{f} 1(\mathrm{ppm})\end{array} & 90 & 80 & 70 & 60 & 50 & 40 & 30 & 20 & 10 & 0 & -10\end{array}$ 
<smiles>CN1C[C@H](c2ccc(C(F)(F)F)cc2)CC1=O</smiles>

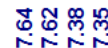<smiles>CN1CCc2ccc(C(F)(F)F)cc2C1</smiles>

4d

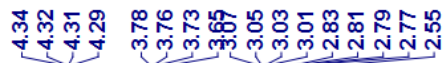

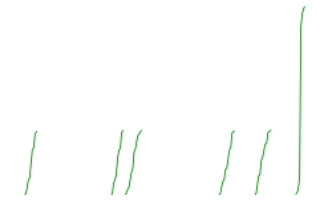

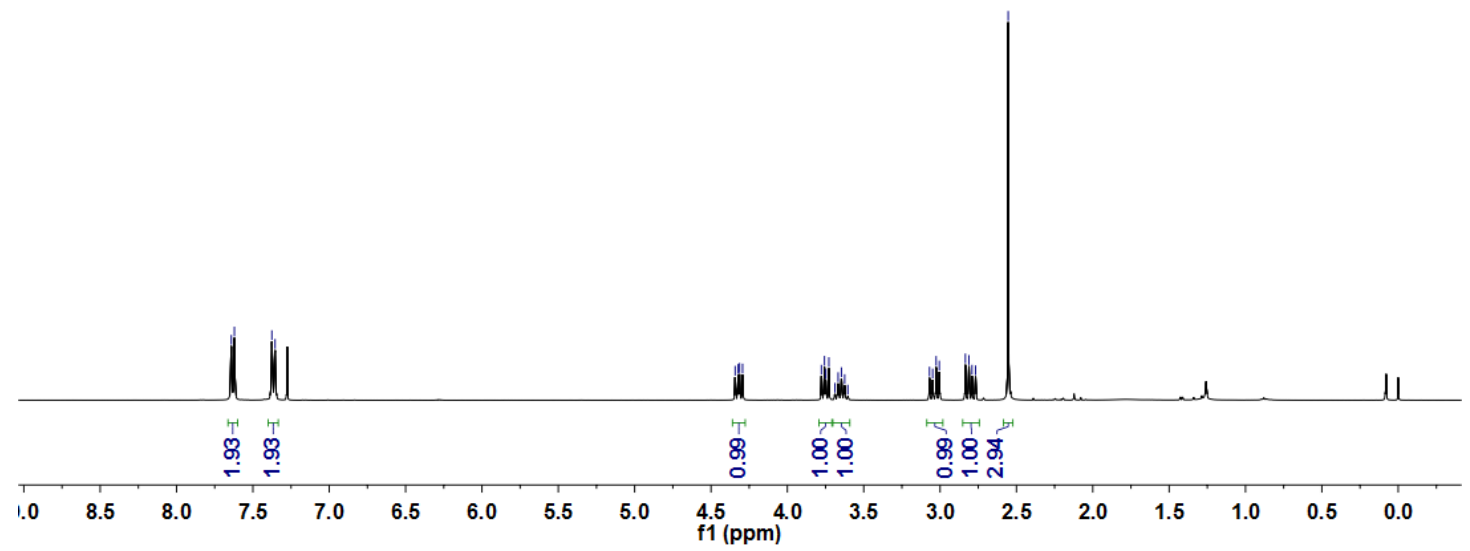

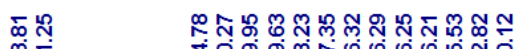

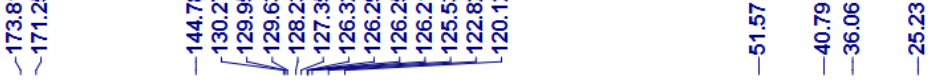<smiles>CN1CCC(Cc2ccc(F)cc2)C1</smiles>

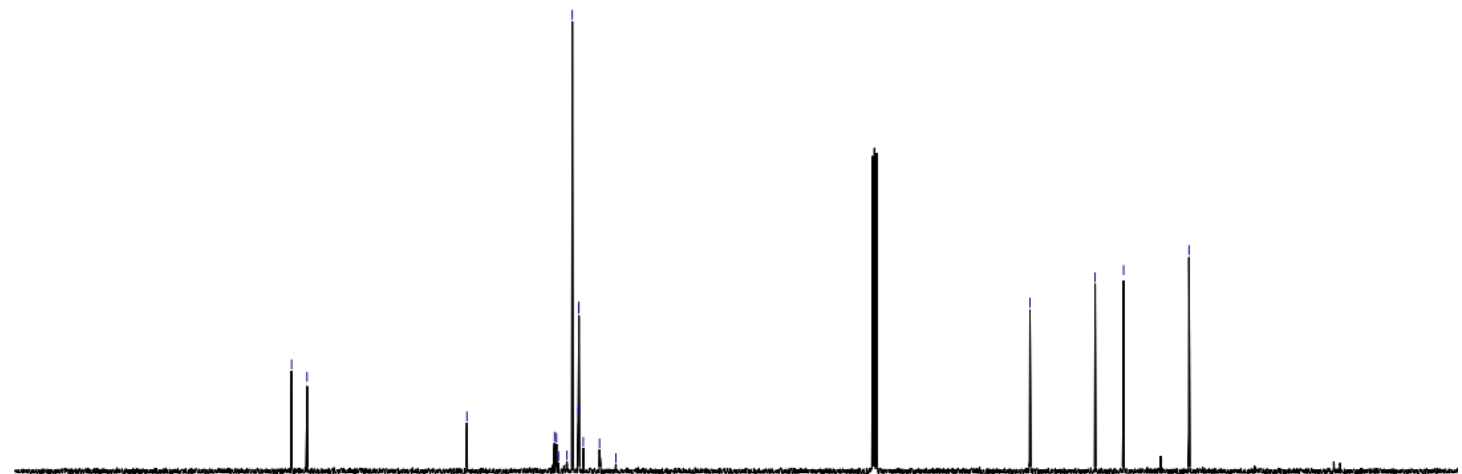

$\begin{array}{llllllllllllllllllllll}210 & 200 & 190 & 180 & 170 & 160 & 150 & 140 & 130 & 120 & 110 \begin{array}{l}100 \\ \mathrm{f} 1(\mathrm{ppm})\end{array} & 90 & 80 & 70 & 60 & 50 & 40 & 30 & 20 & 10 & 0 & -10\end{array}$ 
<smiles>CC(C)(C)N1C[C@H](c2ccc(F)cc2)CC1=O</smiles>

幽

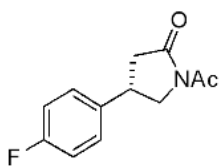

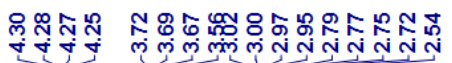

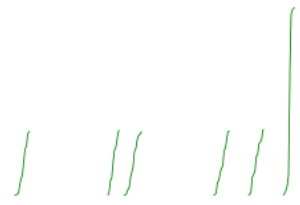

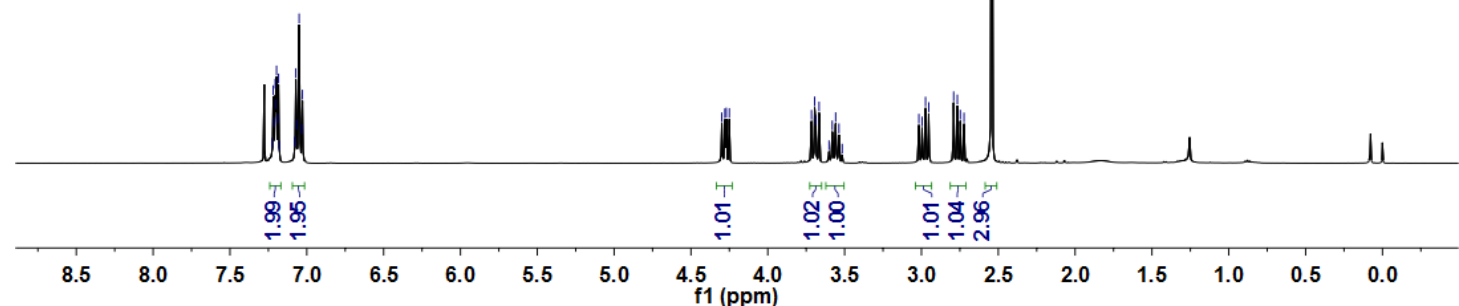

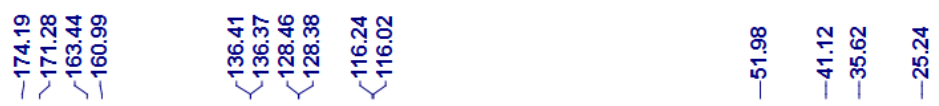
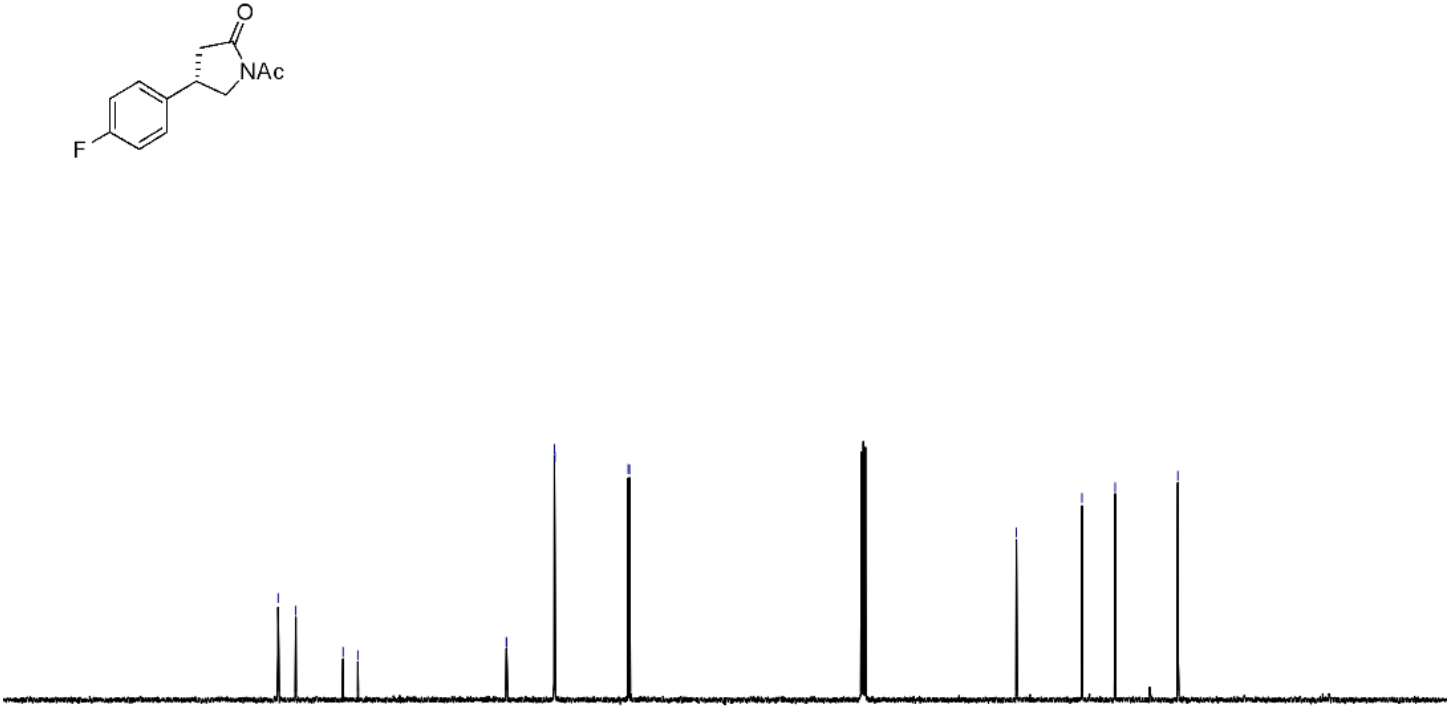

$\begin{array}{llllllllllllllllllllll}210 & 200 & 190 & 180 & 170 & 160 & 150 & 140 & 130 & 120 & 110 \begin{array}{l}100 \\ \mathrm{f} 1(\mathrm{ppm})\end{array} & 90 & 80 & 70 & 60 & 50 & 40 & 30 & 20 & 10 & 0 & -10\end{array}$ 
<smiles>CC(=O)N1C[C@H](c2ccc(Cl)cc2)CC1=O</smiles>

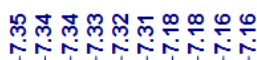

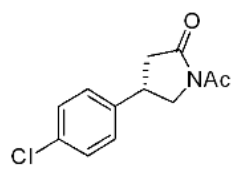

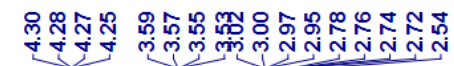

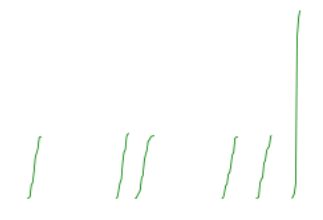

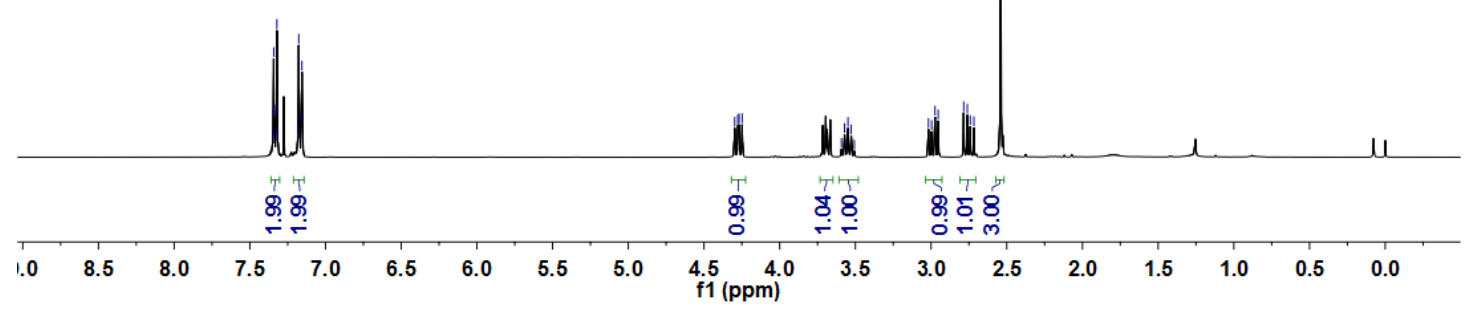

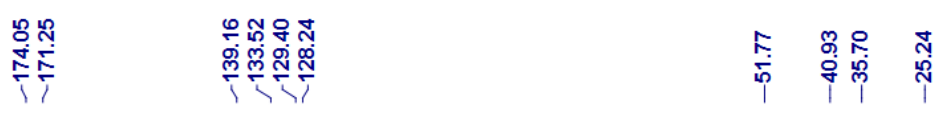

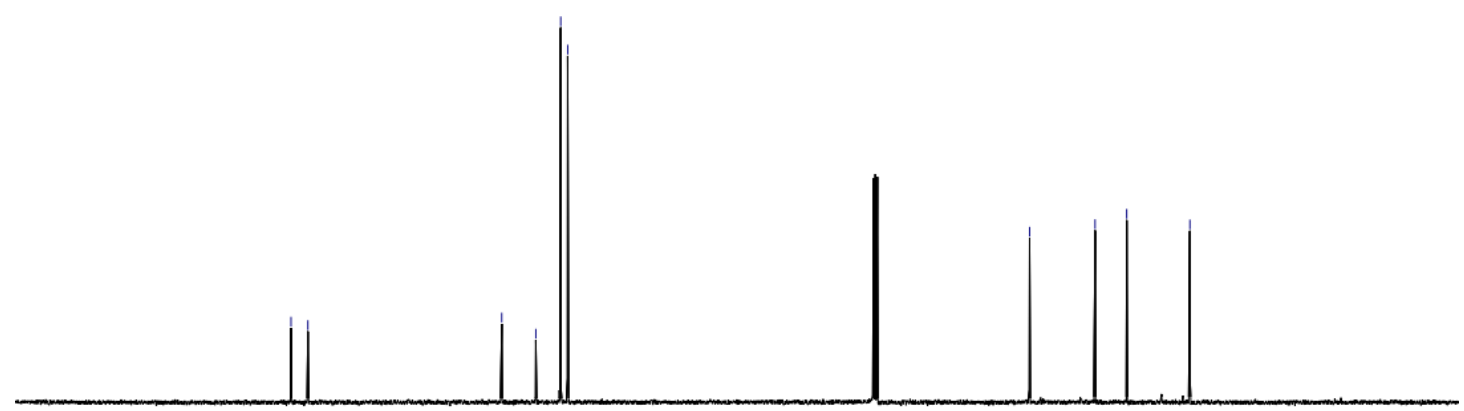

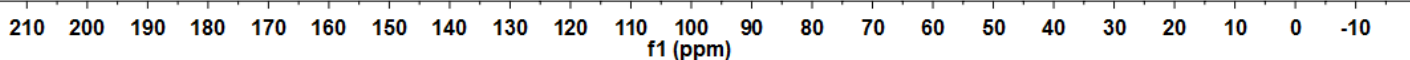



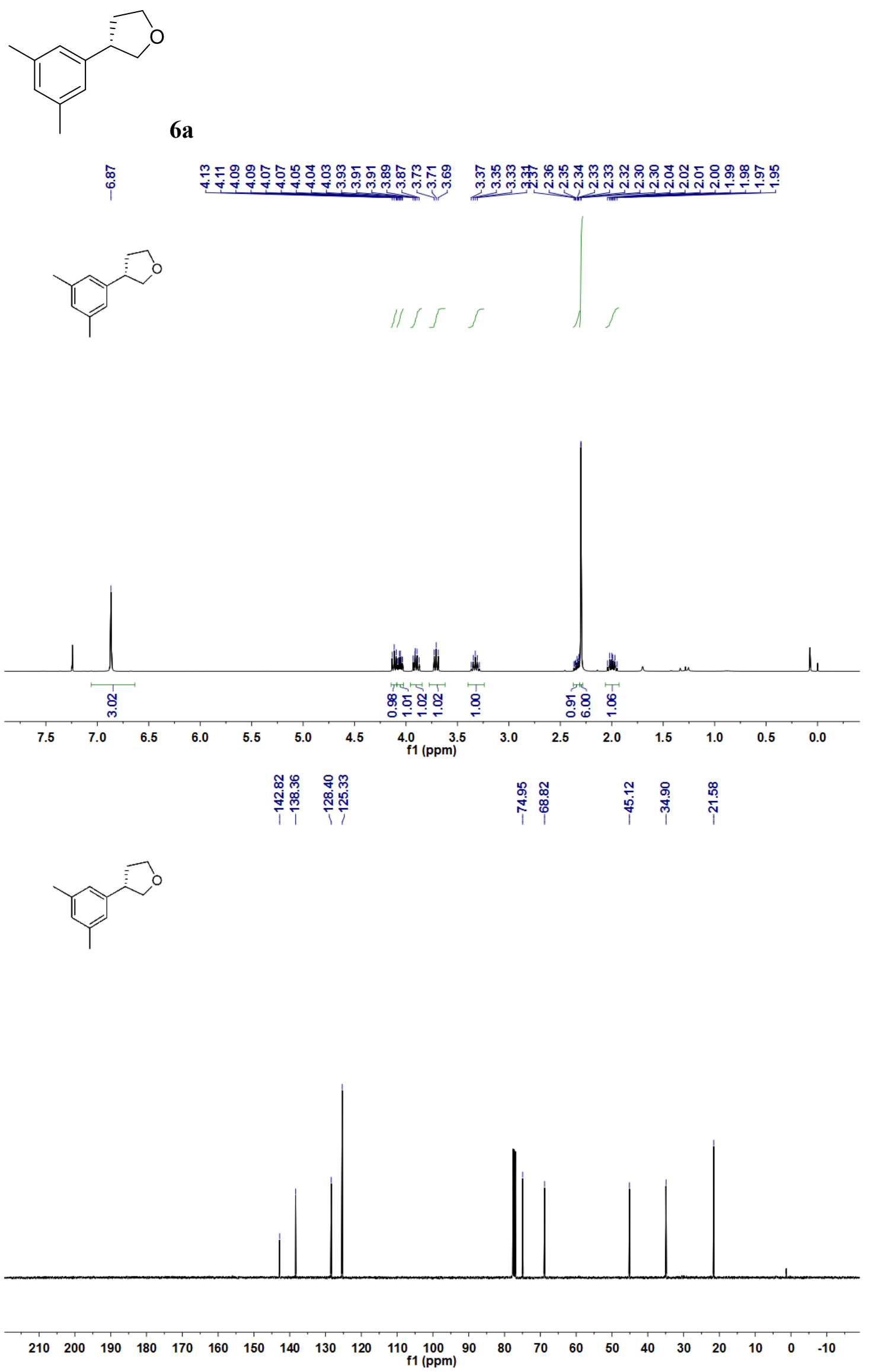

S43 
<smiles>C=CCC1CC(c2cc(C)cc(C)c2)CO1</smiles>

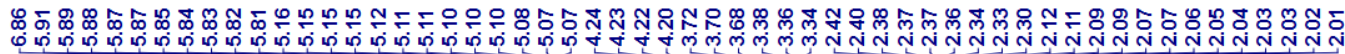<smiles>C=CCC1C=C(Cc2cc(C)cc(C)c2)CC1</smiles>

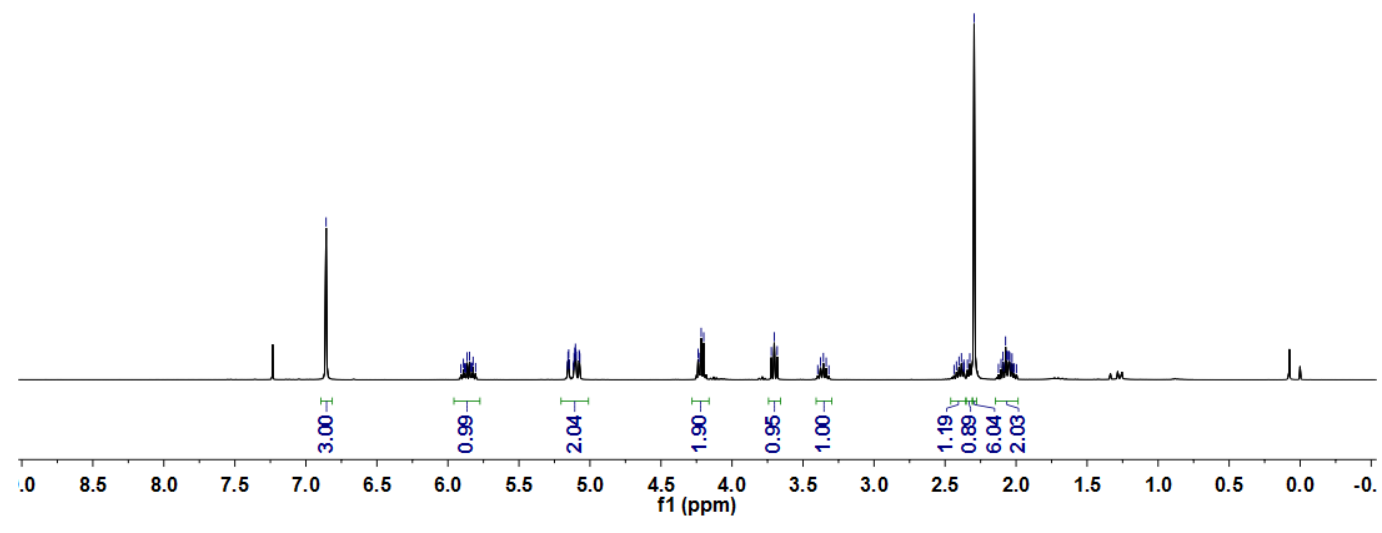

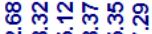

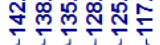

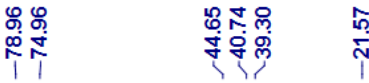<smiles>C=CCC1CC(c2cc(C)cc(C)c2)CO1</smiles>

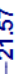

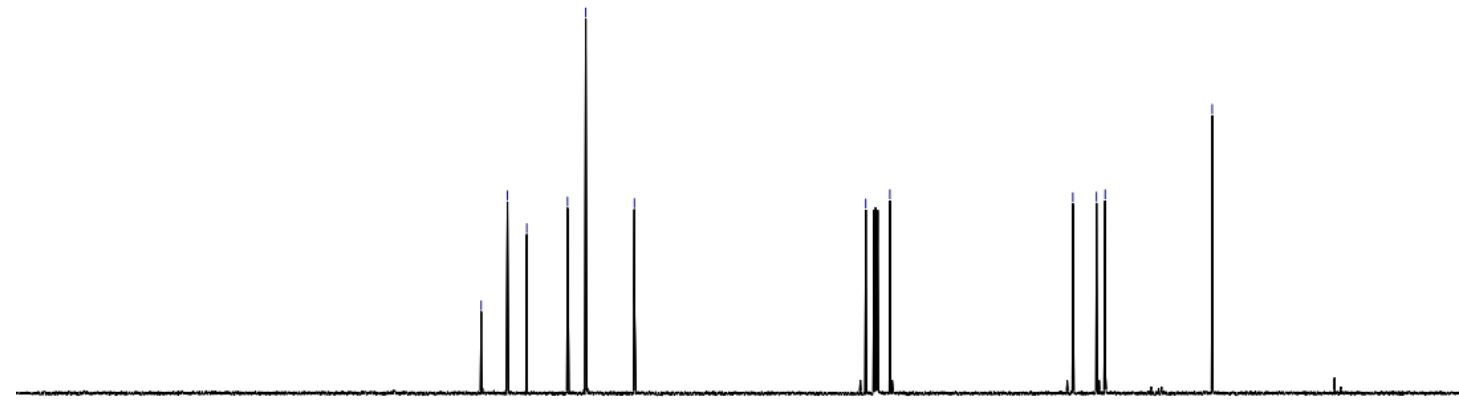

$\begin{array}{lllllllllllllllllllllll}210 & 200 & 190 & 180 & 170 & 160 & 150 & 140 & 130 & 120 & 110 & 100 & 90 & 80 & 70 & 60 & 50 & 40 & 30 & 20 & 10 & 0 & -10\end{array}$ 


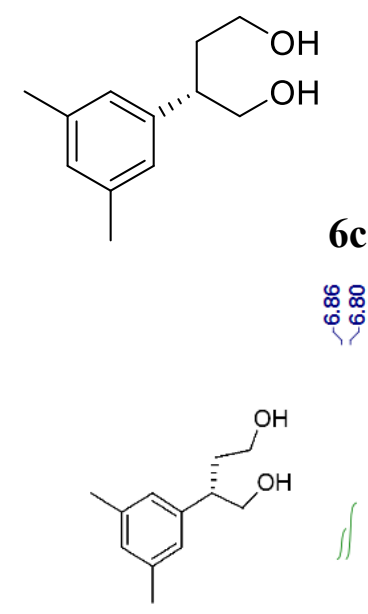

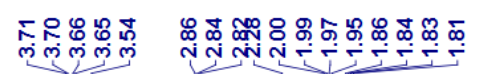

遂
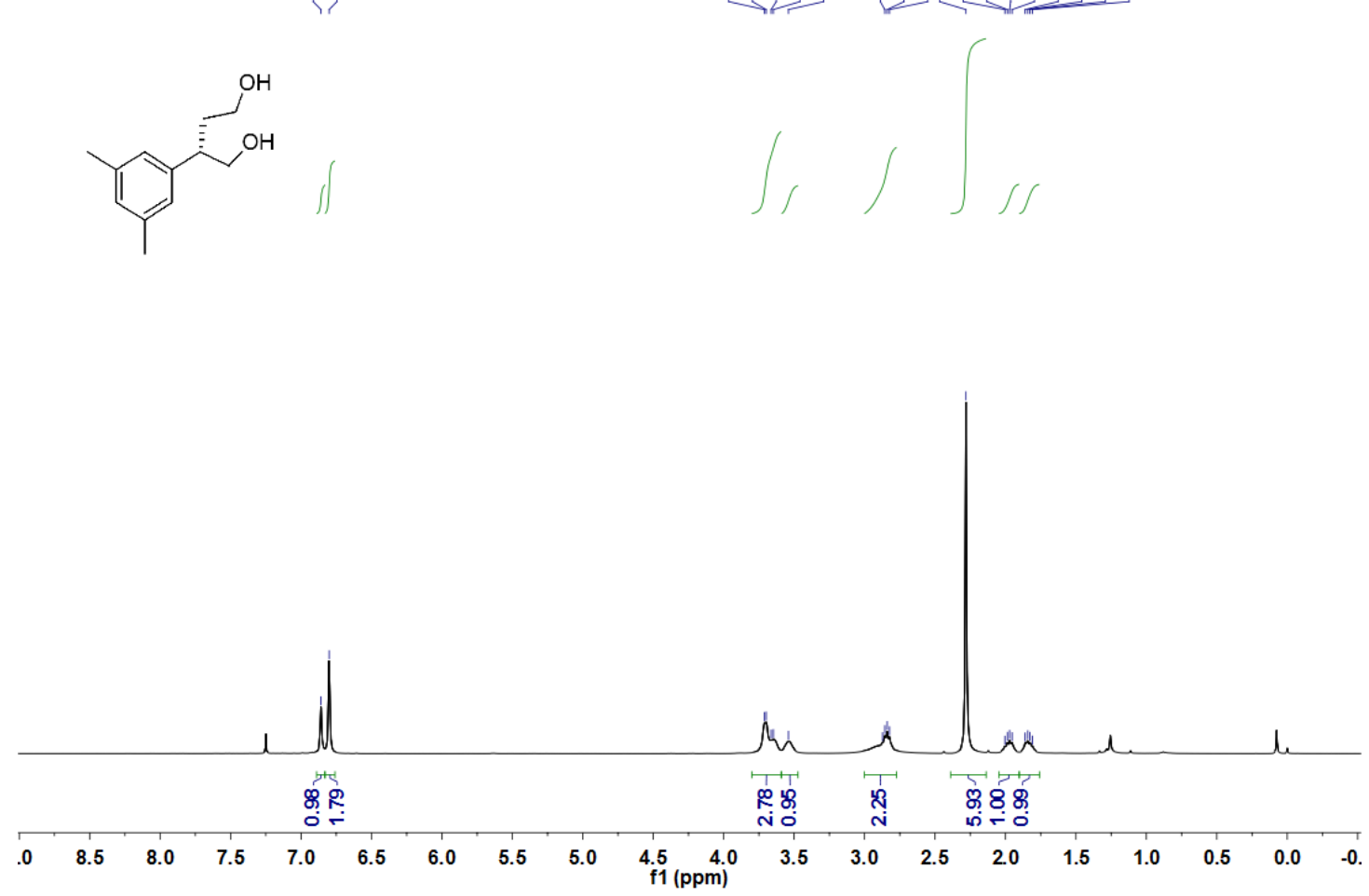

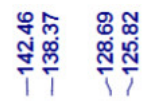

F⿻<smiles>Cc1cc(C)cc(C(CO)CCO)c1</smiles>

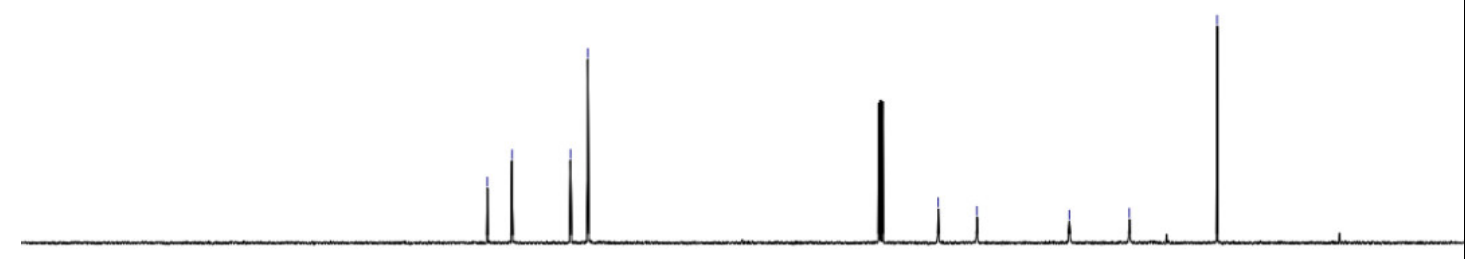

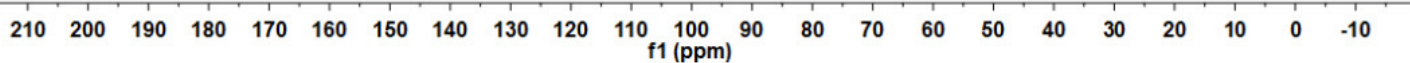


<smiles>COC[C@H](CC=O)c1ccccc1</smiles>
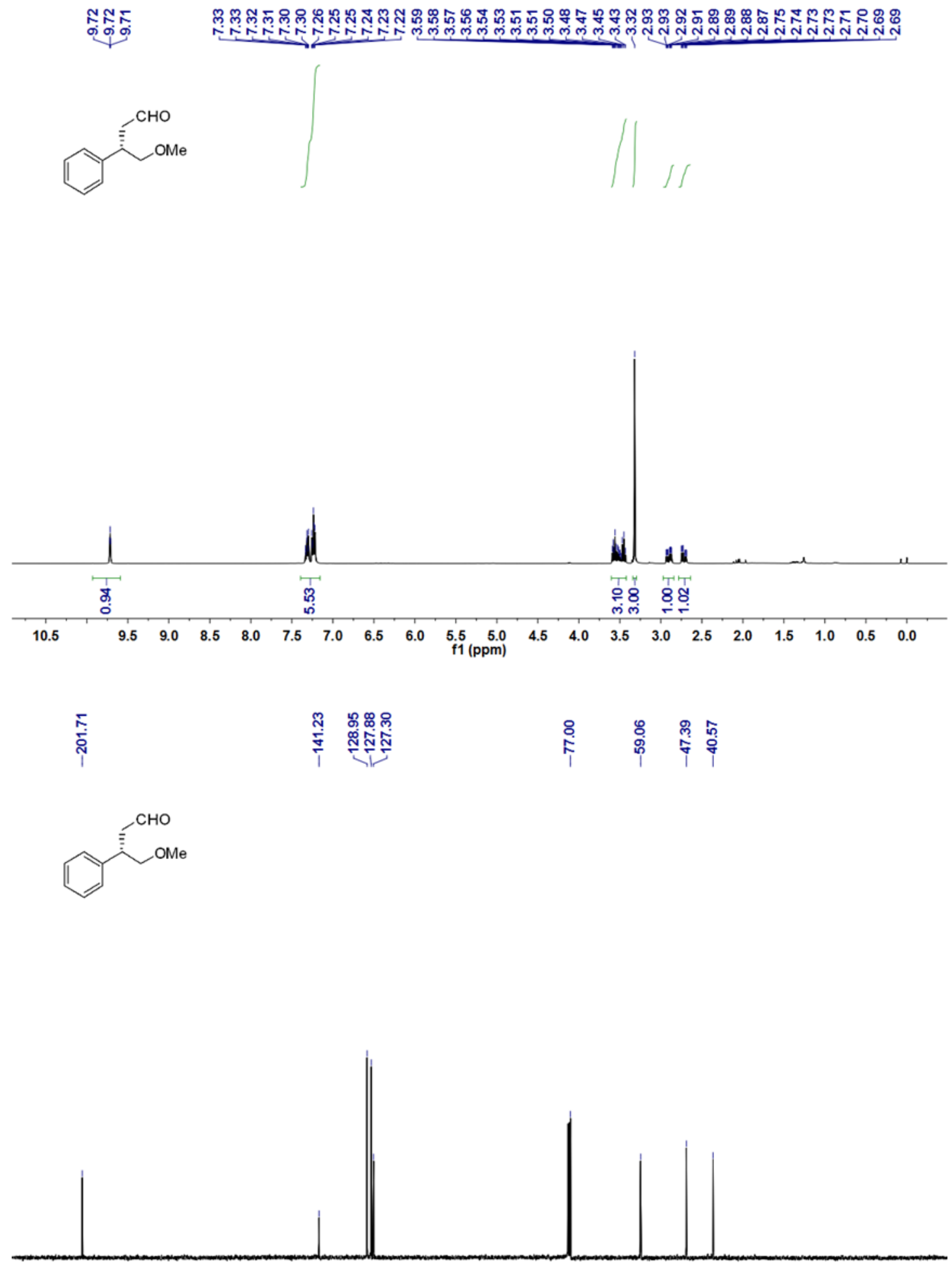

$\begin{array}{lllllllllllllllllllllll}210 & 200 & 190 & 180 & 170 & 160 & 150 & 140 & 130 & 120 & 110 & 100 & 90 & 80 & 70 & 60 & 50 & 40 & 30 & 20 & 10 & 0 & -10\end{array}$ 


\section{HPLC spectra}<smiles>O=C1C[C@H](c2ccccc2)CO1</smiles>

Data File E: \DATA \JX YJX-1-124-0504\YJX-1-124 2018-05-04 16-21-44\025-2001.D Sample Name: LSL-2-170-5

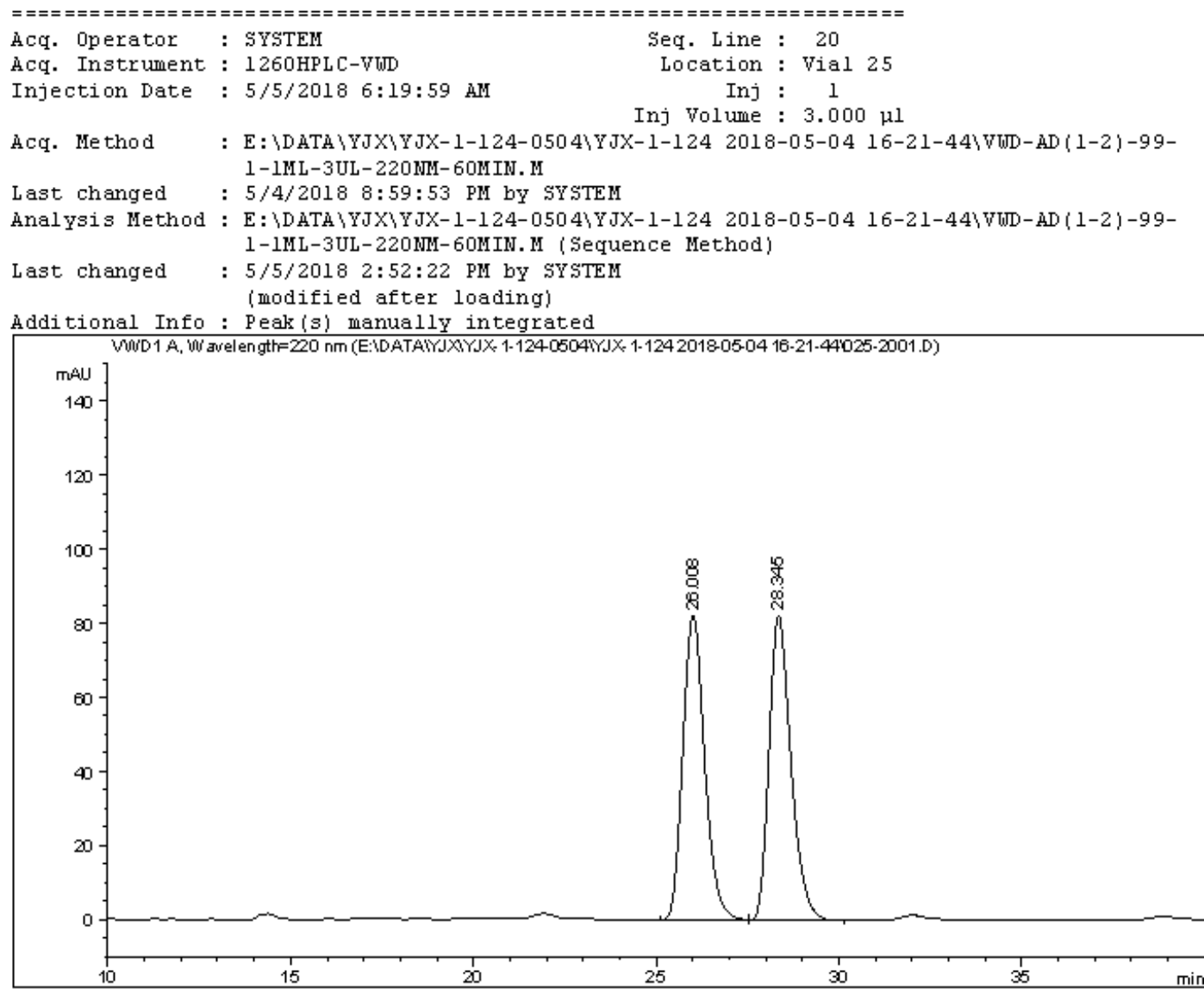

\section{Area Percent Report}

\begin{tabular}{|c|c|}
\hline Sorted BY & : \\
\hline Multiplier & : \\
\hline Dilution & : \\
\hline
\end{tabular}

Do not use Multiplier \& Dilution Factor with ISTD s

Signal 1: VTD $1 \mathrm{~A}$, Wave length $=220 \mathrm{~nm}$

\begin{tabular}{|c|c|c|c|c|c|c|}
\hline $\begin{array}{c}\text { Peak } \\
\#\end{array}$ & $\begin{array}{c}\text { Re tTime } \\
\text { [min] }\end{array}$ & Type & $\begin{array}{l}\text { Width } \\
\text { [min] }\end{array}$ & $\begin{array}{c}\text { Area } \\
\left.\text { [ } \mathrm{MAU} \mathbf{H}^{*} \mathrm{~s}\right]\end{array}$ & $\begin{array}{l}\text { Height } \\
\text { [ } \mathrm{MAU}]\end{array}$ & $\begin{array}{c}\text { Area } \\
\frac{4}{8}\end{array}$ \\
\hline & & & & 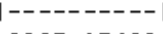 & & \\
\hline 1 & 26.008 & $\mathrm{BB}$ & 0.6422 & 3397.17432 & 82.02698 & 49.8884 \\
\hline 2 & 28.345 & $\mathrm{BB}$ & 0.6335 & 3412.37256 & 82.17161 & 50.1116 \\
\hline ots & $:$ & & & 6809.54688 & 164.19859 & \\
\hline
\end{tabular}

*木* End of Report औ* 
Data File E: \DATA \YJXYYJ-1-124-0504\YJX-1-124 2018-05-04 16-21-44\026-2101.D Sample Name: LSL-2-171-5
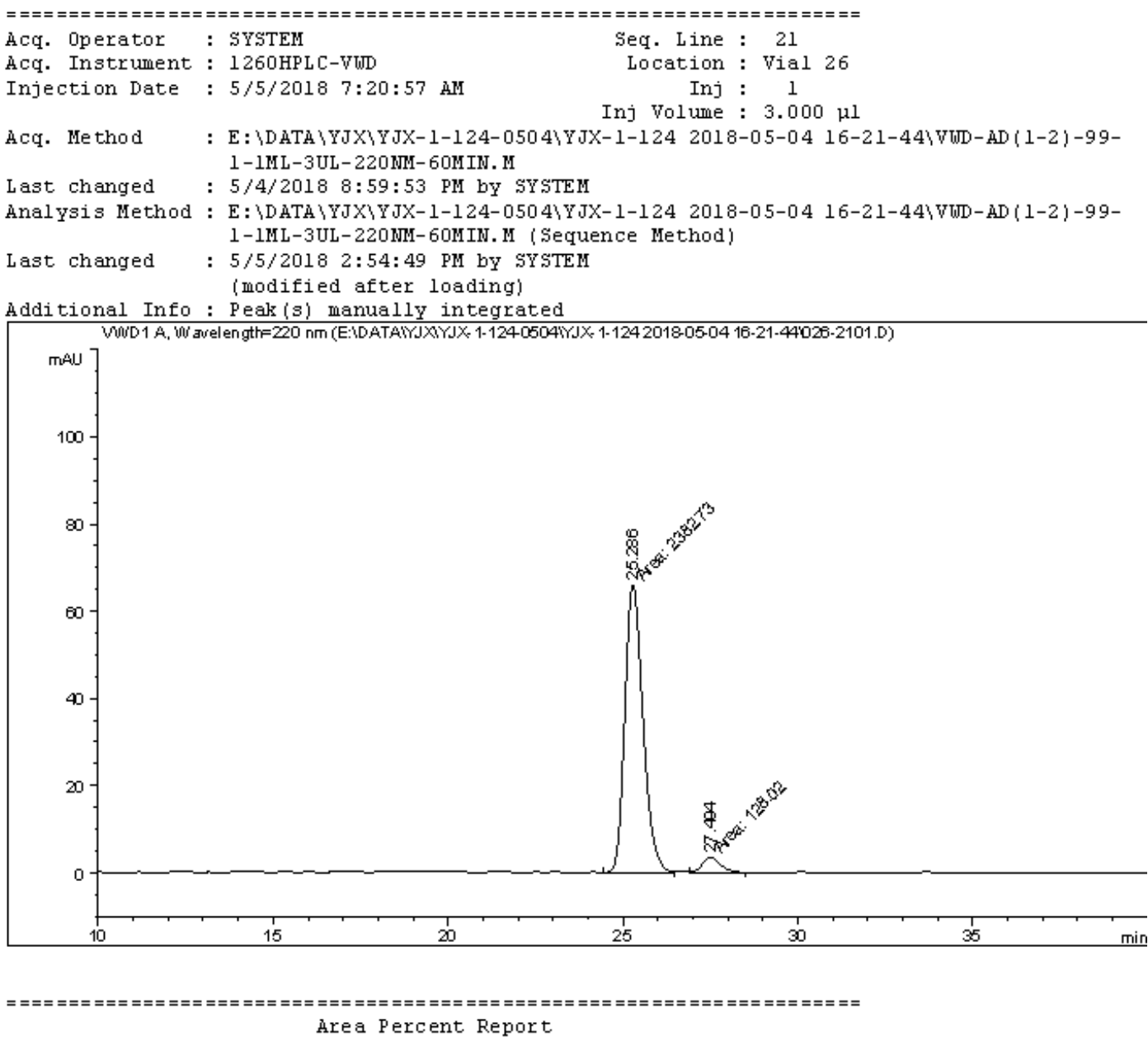

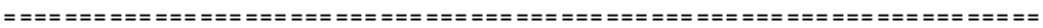

$\begin{array}{llc}\text { Sorted By } & : & \text { Signal } \\ \text { Multiplier } & : & 1.0000 \\ \text { Dilution } & : & 1.0000 \\ \text { Do not use Multiplier } & \& \text { Dilution Factor with ISTD }\end{array}$

Signal 1: VWD $1 \mathrm{~A}$, Wave length $=220 \mathrm{~nm}$

\begin{tabular}{|c|c|c|c|c|c|c|}
\hline $\begin{array}{c}\text { Peak } \\
\#\end{array}$ & $\begin{array}{c}\text { Re tTime } \\
\text { [min] }\end{array}$ & Type & $\begin{array}{l}\text { Width } \\
\text { [min] }\end{array}$ & $\begin{array}{c}\text { Area } \\
{\left[\mathrm{m} A \mathrm{U}^{*} \mathrm{~s}\right]}\end{array}$ & $\begin{array}{l}\text { Height } \\
\text { [ mAU] }\end{array}$ & $\begin{array}{c}\text { Area } \\
\stackrel{4}{*}\end{array}$ \\
\hline & & & & & 6503504 & 94.9011 \\
\hline 1 & $\begin{array}{l}25.286 \\
27.494\end{array}$ & $\begin{array}{l}\mathrm{MM} \\
\mathrm{MM}\end{array}$ & $\begin{array}{l}0.6023 \\
0.6079\end{array}$ & $\begin{array}{r}2382.73022 \\
128.02043\end{array}$ & $\begin{array}{r}65.93504 \\
3.50968\end{array}$ & $\begin{array}{r}94.9011 \\
5.0989\end{array}$ \\
\hline
\end{tabular}

Totals :

$2510.75066 \quad 69.44473$

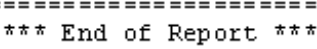


<smiles>Cc1ccc(C2COC(=O)C2)cc1</smiles>

Data File E: \DATA \LSLLSL-2-143\LSL-2-143 2018-04-06 12-13-03\002-0301.D Sample Name: LSL-2-117

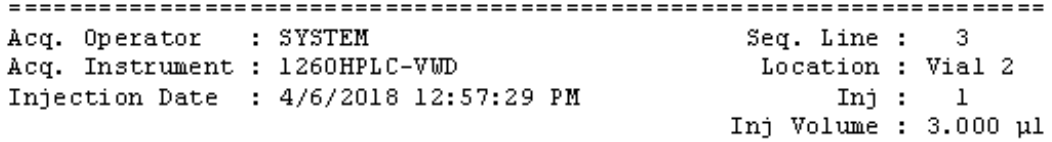

Acq. Me thod : E: \DATA \LSL \LSL-2-143\LSL-2-143 2018-04-06 12-13-03 VTD-AD (1-2)-97-3-1ML $-3 \mathrm{UL}-22$ OMM $-30 \mathrm{MIN}$. M

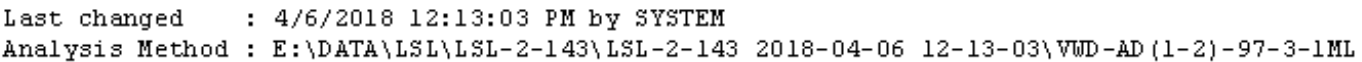
-3 UL -22 OMM-30MIN.M (Sequence Me thod)

Last changed : 4/30/2018 10:41:01 AM by 5YsTEM

(modified after loading)

Additional Info : Peak (s) manually integrated

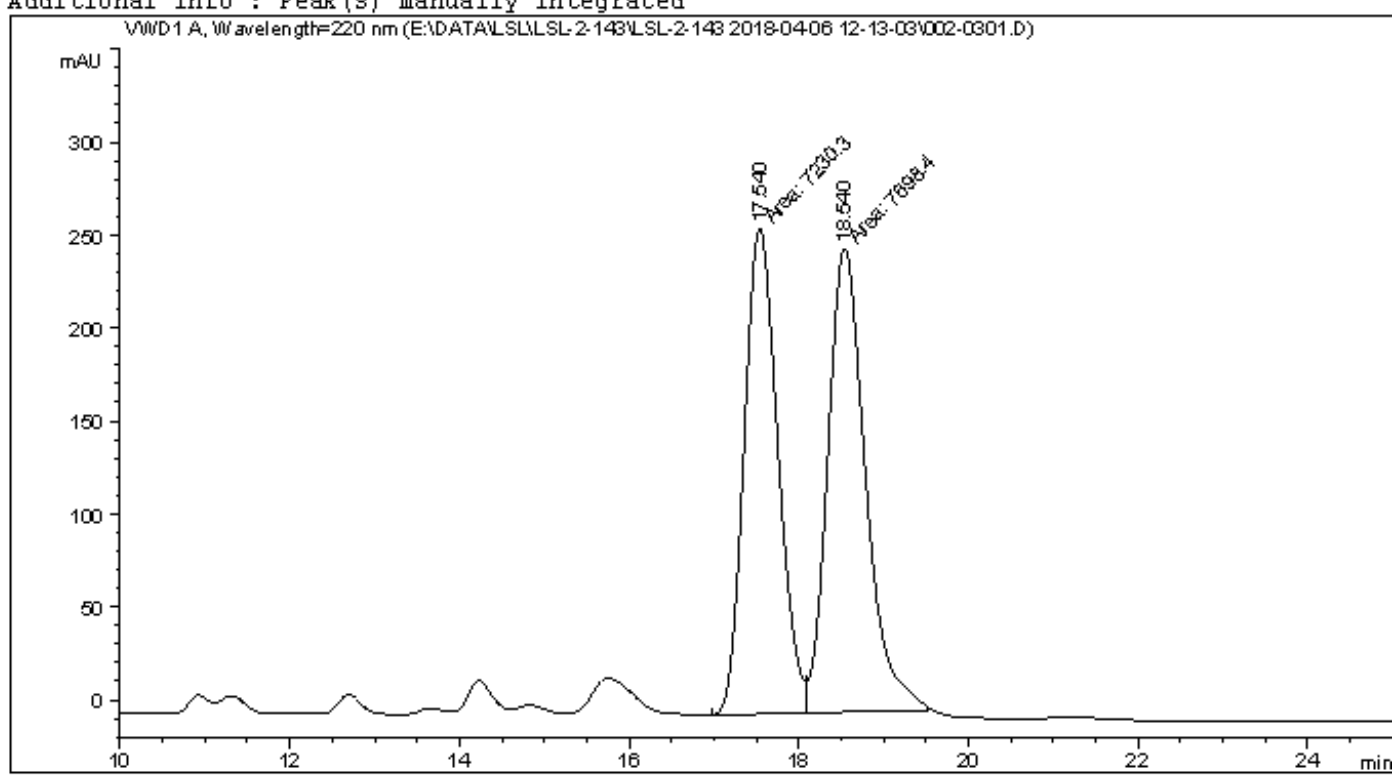

Area Percent Report

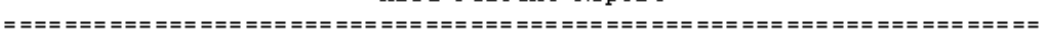

$\begin{array}{lll}\text { Sorted By } & : & \text { Signal } \\ \text { Multiplier } & : & 1.0000 \\ \text { Dilution } & : & 1.0000\end{array}$

Dilution : l.0000

Do not use Multiplier \& Dilution Factor with ISTD s

Signal 1: VWD $1 \mathrm{~A}$, Wave length $=220 \mathrm{~nm}$

\begin{tabular}{|c|c|c|c|c|c|c|}
\hline $\begin{array}{c}\text { Peak } \\
\#\end{array}$ & $\begin{array}{c}\text { Re tTime } \\
\text { [min] }\end{array}$ & Type & $\begin{array}{l}\text { Width } \\
\text { [min] }\end{array}$ & $\begin{array}{c}\text { Area } \\
{\left[\mathrm{m} A U^{*} s\right]}\end{array}$ & $\begin{array}{l}\text { Height } \\
\text { [ [mAU] }\end{array}$ & $\begin{array}{c}\text { Area } \\
\text { s }\end{array}$ \\
\hline 1 & 17.540 & MF & 0.4616 & 7230.30078 & 261.08081 & 48.4322 \\
\hline 2 & 18.540 & FM & 0.5146 & 7698.40234 & 249.33826 & 51.5678 \\
\hline
\end{tabular}

Totals : $\quad$ 1. $49287 \mathrm{e} 4 \quad 510.41907$ 
Data File E: \DATA \SL \LL-2-143\LSL-2-143 2018-04-06 12-13-03\003-0401.D Sample Name: LSL-2-143

\begin{tabular}{|c|c|c|c|}
\hline Acq. Operator & : SYSTEM & Seq. Line & 4 \\
\hline Acq. Instrument & : 1260HPLC-VWD & Location : & : Vial 3 \\
\hline Injection Date & : 4/6/2018 1:28:14 PM & $\begin{array}{r}\text { Inj } \\
\text { Inj Volume }\end{array}$ & $\begin{array}{cc}: & 1 \\
: & 3.000\end{array}$ \\
\hline
\end{tabular}

Acq. Me thod : E: \DATA \LSL \L SL-2-143\LSL-2-143 2018-04-06 12-13-03 VWD-AD (1-2)-97-3-1ML $-3 \mathrm{UL}-22$ OMM-30MIN.M

Last changed : 4/6/2018 12:13:03 PM by SYSTEM

Analysis Method : E: \DATA \LSL \LL-2-143\LSL-2-143 2018-04-06 12-13-03 VWD-AD (1-2)-97-3-1ML -3UL-220MM-30MIN.M (Sequence Me thod)

Last changed : 4/30/2018 10:45:29 AM by SYSTEM

(modified after loading)

Additional Info : Peak (s) manually integrated
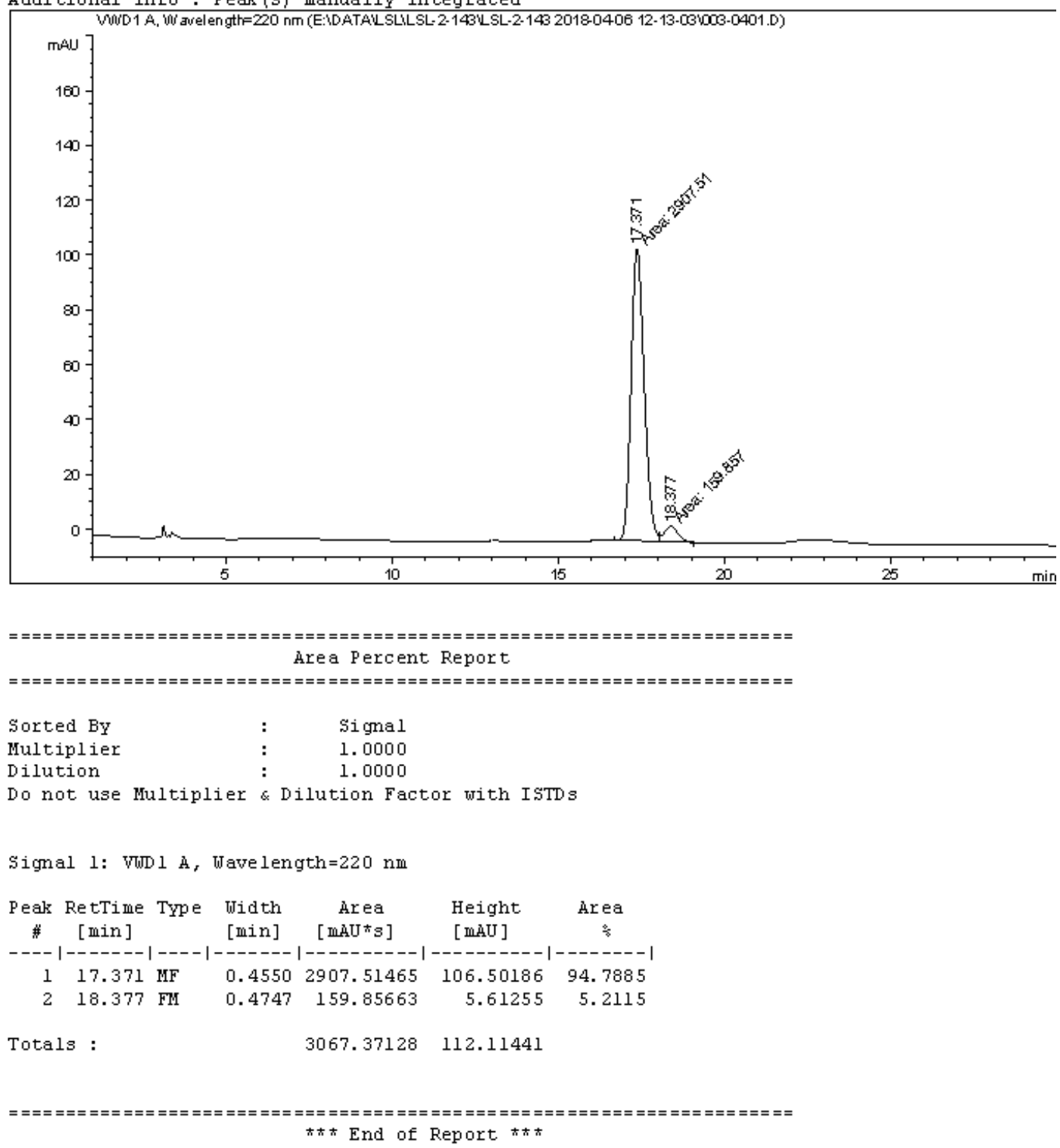
<smiles>CC(C)c1ccc(C2COC(=O)C2)cc1</smiles>

2c

Data File E: \DATA \WSW LUD-3-185\LWD-3-185 2018-04-09 10-26-44\003-0701.D Sample Name: LSL-2-152-2

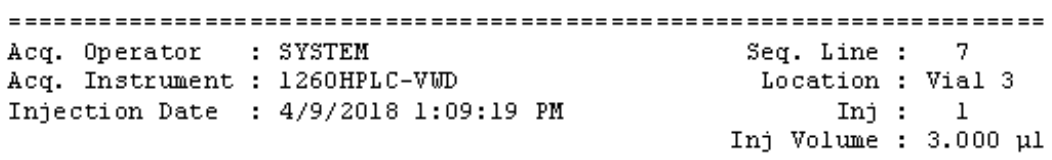

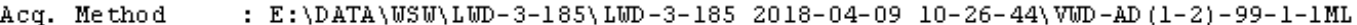
$-3 \mathrm{UL}-22$ ONM $-60 \mathrm{MIN}$. M

Last changed : 4/9/2018 11:50:21 AM bY SYSTEM

Analysis Method : E: \DATA \USW\LWD-3-185 LWD-3-185 2018-04-09 10-26-44\VWD-AD (1-2)-99-1-1ML $-3 \mathrm{UL}-220 \mathrm{MM}-60 \mathrm{MIN}$.M (Sequence Me thod)

Last changed : 4/30/2018 10:59:08 AM by SYSTEM

(modified after loading)

Additional Info : Peak (s) manually integrated

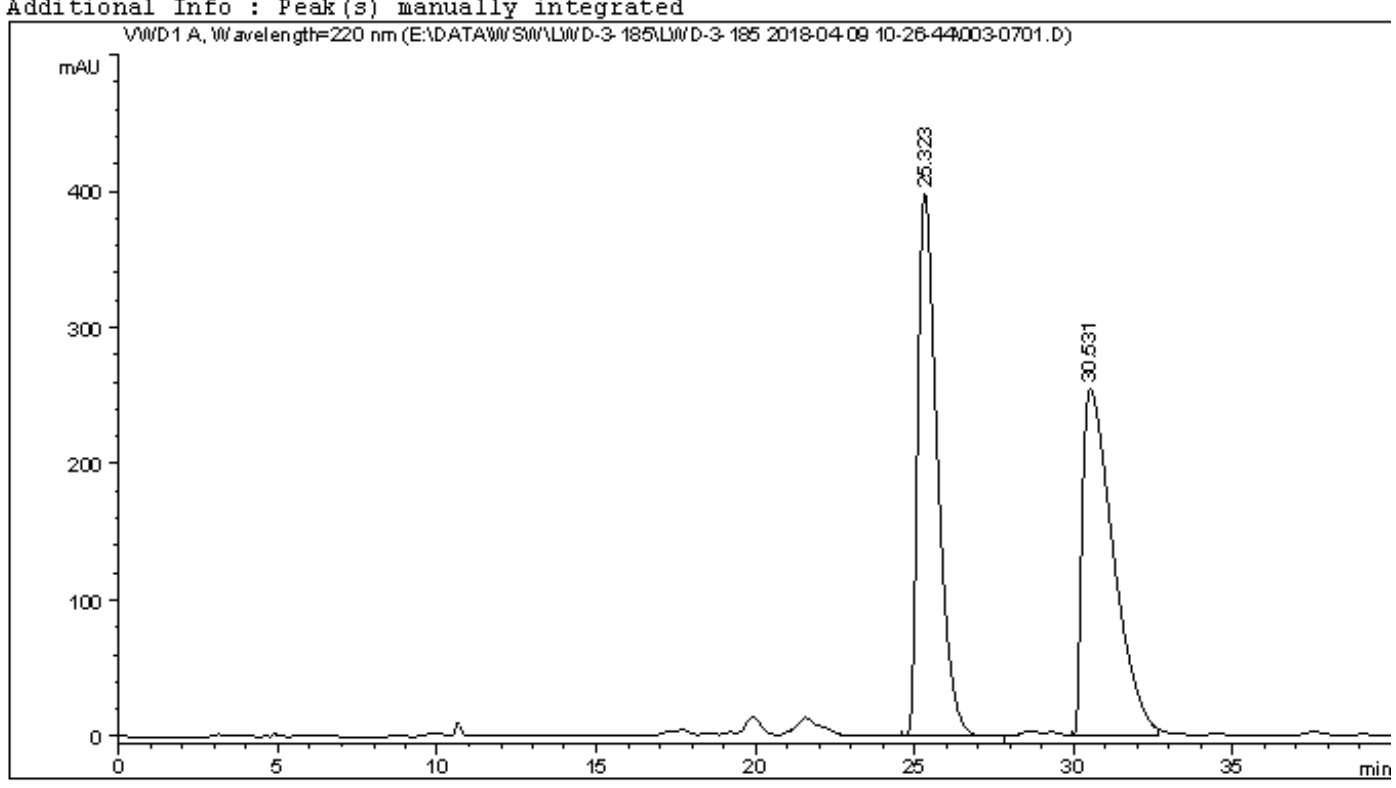

\section{Area Percent Report}

\begin{tabular}{|c|c|c|}
\hline Sorted By & : & Sign \\
\hline Multiplier & : & 1.00 \\
\hline Dilution & $:$ & 1.000 \\
\hline
\end{tabular}

Do not use Multiplier \& Dilution Factor with ISTDs

Signal 1: VWD 1 A, Wave length $=220 \mathrm{~nm}$

\begin{tabular}{|c|c|c|c|c|c|c|}
\hline $\begin{array}{c}\text { Peak } \\
\#\end{array}$ & $\begin{array}{l}\text { Re tTime } \\
\text { [min] }\end{array}$ & Type & $\begin{array}{l}\text { Width } \\
\text { [min] }\end{array}$ & $\begin{array}{c}\text { Area } \\
{\left[\mathrm{mAU} U^{*} \Omega\right]}\end{array}$ & $\begin{array}{l}\text { Height } \\
\text { [ } \mathrm{mAU}]\end{array}$ & $\begin{array}{c}\text { Area } \\
\frac{5}{*}\end{array}$ \\
\hline$=--$ & & & & -------- & $-0-1-1$ & ------1 \\
\hline 1 & 25.323 & $\mathrm{BB}$ & 0.6573 & 1. $70622 \mathrm{e} 4$ & 397.89001 & 49.8637 \\
\hline 2 & 30.531 & $\mathrm{BV}$ & 1.0422 & 1. $71555 \mathrm{e} 4$ & 254.58685 & 50.1363 \\
\hline Tota. & : & & & 3. $42177 \mathrm{e} 4$ & 652.47687 & \\
\hline
\end{tabular}

\#* End of Report ** 
Data File E: \DATA $\backslash$ LS \LSL-2-156\LSL-2-156 2018-04-14 21-53-52\012-0301.D Sample Name: LSL-2-156-2

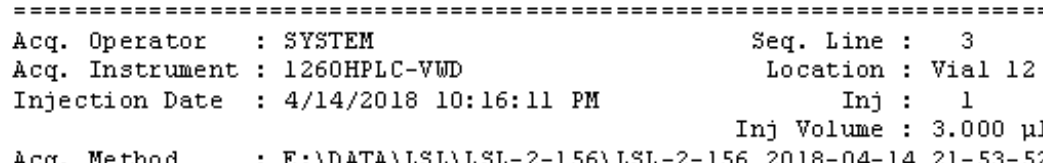

Acq. Me thod : E: \DATA \LSL \LSL-2-156\LSL-2-156 2018-04-14 21-53-52 VWD-AD (1-2)-99-1-1ML $-3 \mathrm{UL}-22$ OMM-60MIN.M

Last changed : 4/14/2018 9:53:53 PM by SYSTEM

Analysis Method : E: \DATA \LSL \LL-2-156\LSL-2-156 2018-04-14 21-53-52 VWD-AD (1-2)-99-1-1ML $-3 \mathrm{UL}-220 \mathrm{MM}-60 \mathrm{MIN}$.M (Sequence Me thod)

Last changed : 4/30/2018 10:51:50 AM by SYSTEM

(modified after loading)

Additional Info : Peak (s) manually integrated
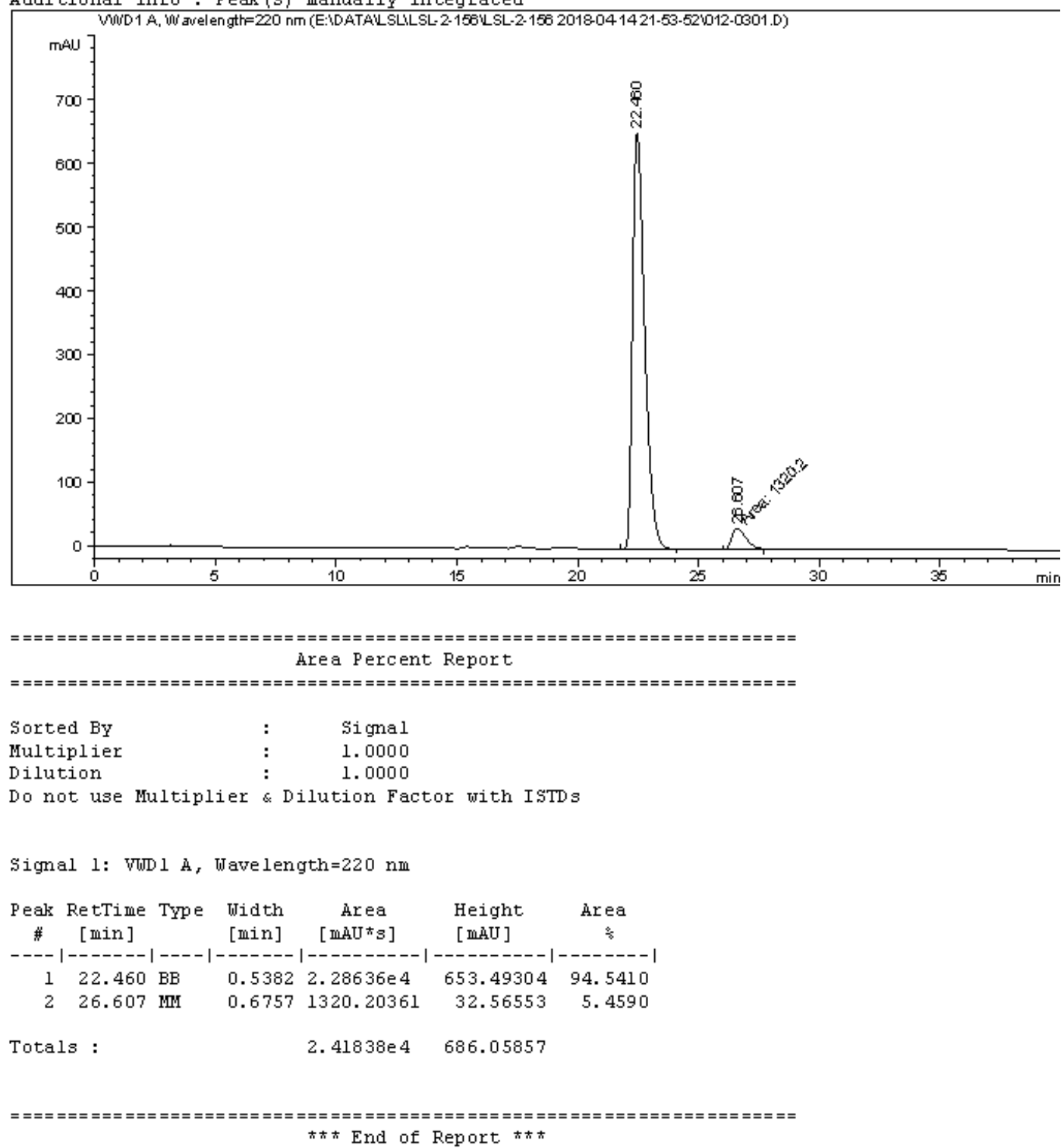
<smiles></smiles>

Data File E: \DATA \LGY Sample Name: LSL-2-162-1
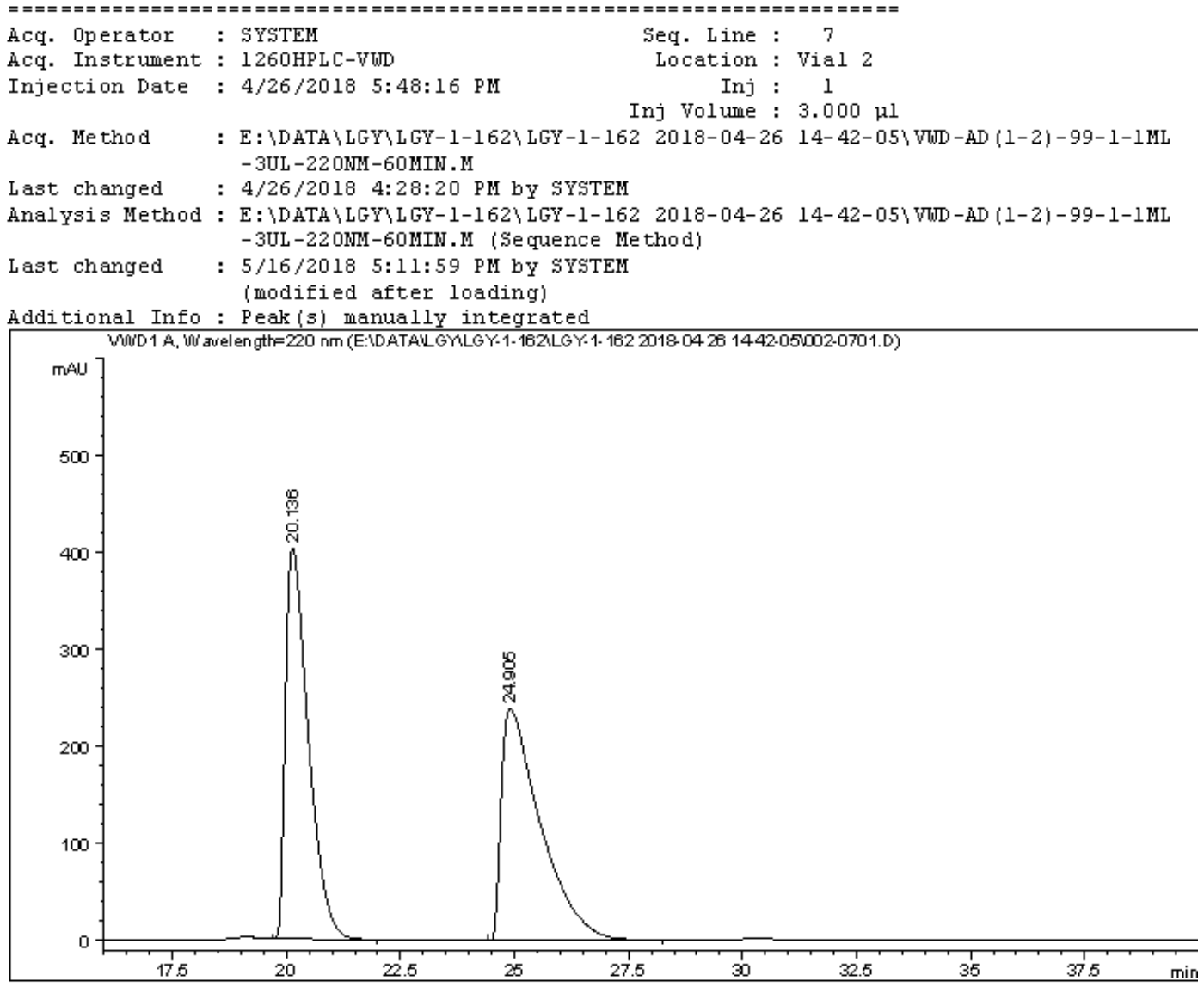

Area Percent Report

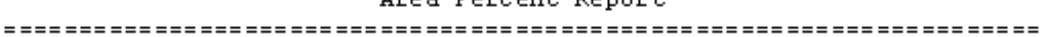

$\begin{array}{lll}\text { Sorted BY } & : & \text { Signal } \\ \text { Multiplier } & : & 1.0000 \\ \text { Dilution } & : & 1.0000\end{array}$

Dilution : 1.0000

Do not use Multiplier \& Dilution Factor with ISTD s

Signal 1: VWD 1 A, Wave length $=220 \mathrm{~nm}$

\begin{tabular}{|c|c|c|c|c|c|c|}
\hline $\begin{array}{c}\text { Peak } \\
\#\end{array}$ & $\begin{array}{c}\text { RetTime } \\
\text { [min] }\end{array}$ & Type & $\begin{array}{l}\text { Width } \\
\text { [min] }\end{array}$ & $\begin{array}{c}\text { Area } \\
{\left[\mathrm{mAU}^{*} \mathrm{~s}\right]}\end{array}$ & $\begin{array}{l}\text { Height } \\
\text { [ [MAU] }\end{array}$ & Area \\
\hline 1 & 20.136 & $\mathrm{BB}$ & 0.5281 & 1. $40234 \mathrm{e} 4$ & 403.87665 & 49.7834 \\
\hline 2 & 24.905 & $\mathrm{BB}$ & 0.8622 & 1. $41454 \mathrm{e} 4$ & 237.73882 & 50.2166 \\
\hline ota & : & & & $2.81688 \mathrm{e} 4$ & 641.61546 & \\
\hline
\end{tabular}


Data File E: \DATA LGY LGY-1-162\LGY-1-162 2018-04-26 14-42-05\003-0801.D Sample Name: LSL-2-166-1

\begin{tabular}{|c|c|c|c|}
\hline $\begin{array}{l}\text { Acq. Operator } \\
\text { Acq. Instrument }\end{array}$ & $\begin{array}{l}: \text { SYSTEM } \\
: 1260 \mathrm{HPLC} \text {-VUD }\end{array}$ & $\begin{array}{l}\text { Seq. Line } \\
\text { Location }\end{array}$ & $\begin{array}{c}: \\
: \text { Vial } 3\end{array}$ \\
\hline Injection Date & : 4/26/2018 6:49:03 PM & $\begin{array}{r}\text { Inj } \\
\text { Inj Volume }\end{array}$ & $\begin{array}{l}: \quad 1 \\
: 3.000\end{array}$ \\
\hline
\end{tabular}

Acq. Me thod : E: \DATA \LGY \LGY-1-162\LGY-1-162 2018-04-26 14-42-05 VWD-AD (1-2)-99-1-1ML $-3 \mathrm{UL}-22$ OMM-60MIN.M

Last changed : 4/26/2018 4:28:20 PM by SYSTEM

Analysis Method : E: \DATA \LGY -3UL-22 ONM-60MIN.M (Sequence Me thod)

Last changed : 5/16/2018 5:13:54 PM by SYSTEM

(modified after loading)

Additional Info : Peak (s) manually integrated
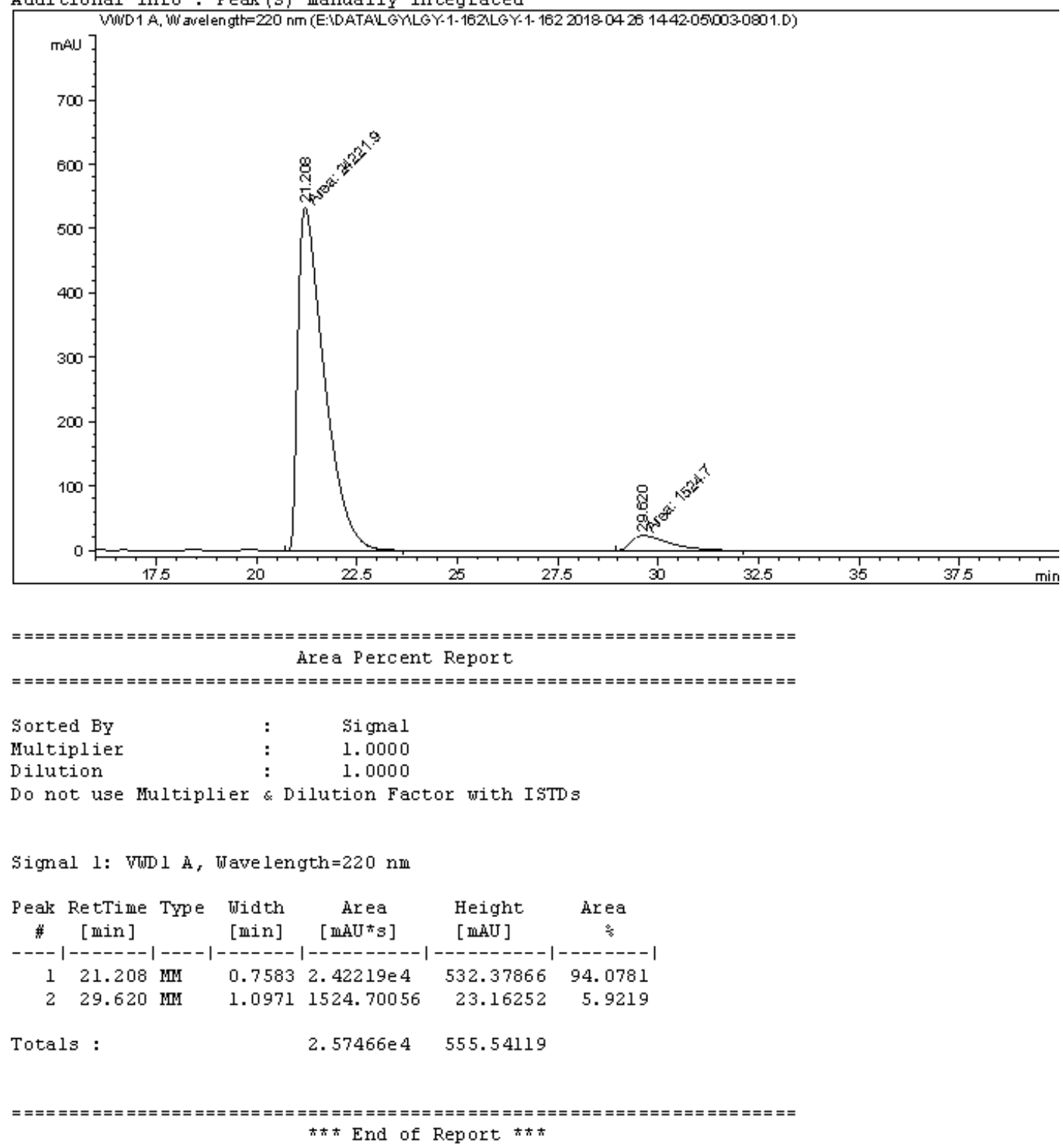
<smiles>O=C1C[C@H](c2ccc(-c3ccccc3)cc2)CO1</smiles>

Data File E: \DATA \LSL \LL-2-166\LSL-2-166-2 2018-05-02 15-04-51\001-0301.D Sample Name: LSL-2-162-2

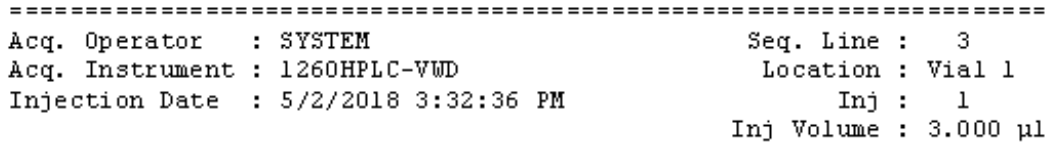

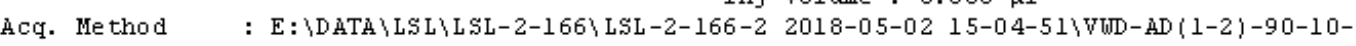
$1 \mathrm{ML}-3 \mathrm{UL}-254 \mathrm{MM}-30 \mathrm{MIN}$. M

Last changed : 5/2/2018 3:04:52 PM by SYSTEM

Analysis Method : E: \DATA \LSL \LSL-2-166\LSL-2-166-2 2018-05-02 15-04-51 VWD-AD (1-2)-90-10$1 \mathrm{ML}-3 \mathrm{UL}-254 \mathrm{NM}-30 \mathrm{MIN}$.M (Sequence Me thod)

Last changed : 5/8/2018 11:06:46 AM bY SYSTEM (modified after loading)

Additional Info : Peak ( $s$ ) manually integrated

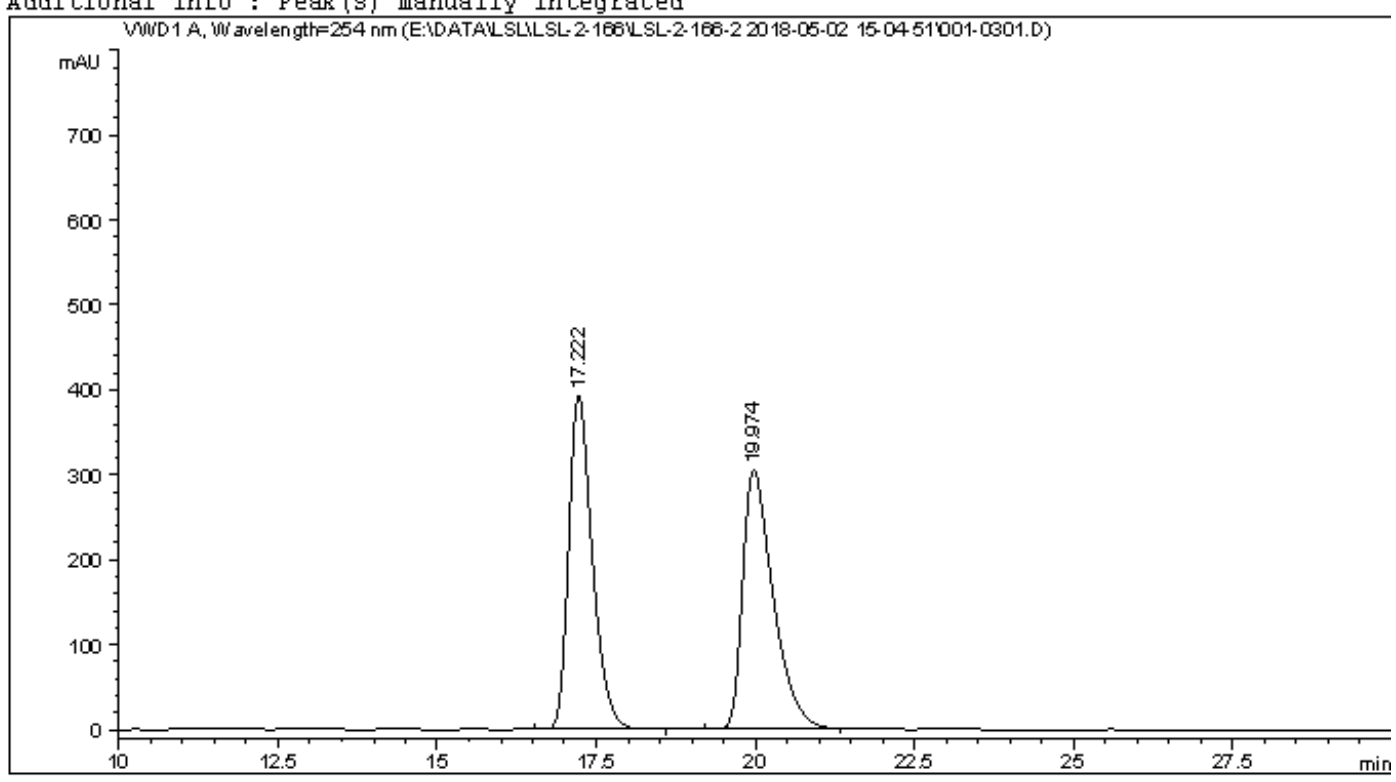

Area Percent Report

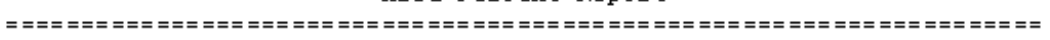

$\begin{array}{lll}\text { Sorted BY } & : & \text { Signal } \\ \text { Multiplier } & : & 1.0000 \\ \text { Dilution } & : & 1.0000\end{array}$

Dilution : 1.0000

Do not use Multiplier \& Dilution Factor with ISTD s

Signal 1: VWD 1 A, Wave length $=254 \mathrm{~nm}$

\begin{tabular}{|c|c|c|c|c|c|c|}
\hline $\begin{array}{c}\text { Peak } \\
\#\end{array}$ & $\begin{array}{c}\text { Re tTime } \\
\text { [min] }\end{array}$ & Type & $\begin{array}{l}\text { Width } \\
\text { [min] }\end{array}$ & $\begin{array}{c}\text { Area } \\
{\left[\mathrm{mAU}^{*} \mathrm{~s}\right]}\end{array}$ & $\begin{array}{l}\text { Height } \\
\text { [ [MAU] }\end{array}$ & $\begin{array}{c}\text { Area } \\
\stackrel{5}{*}\end{array}$ \\
\hline & & & & & & \\
\hline 1 & 17.222 & BB & 0.3922 & $1.01572 \mathrm{e} 4$ & 393.39670 & 50.0595 \\
\hline 2 & 19.974 & BB & 0.4988 & 1. $01330 \mathrm{e} 4$ & 304.88220 & 49.9405 \\
\hline $0 t$ & & & & $2.02902 \mathrm{e} 4$ & 7890 & \\
\hline
\end{tabular}

ネネー End of Report $ネ$ ה 
Data File E: \DATA \LS \LSL-2-166\LSL-2-166-2 2018-05-02 15-04-51\002-0401.D Sample Name: LSL-2-166-2

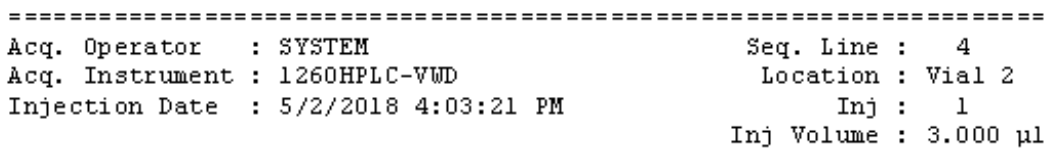

Acq. Me thod : E: \DATA \LSL \LSL-2-166\LSL-2-166-2 2018-05-02 15-04-51 VWD-AD(1-2)-90-10$1 \mathrm{ML}-3 \mathrm{UL}-254 \mathrm{NM}-30 \mathrm{MIN}$. M

Last changed : 5/2/2018 3:04:52 PM by SYTTE

Analysis Method : E: \DATA \LSL \SL-2-166\LSL-2-166-2 2018-05-02 15-04-51\VWD-AD(1-2)-90-10$1 M L-3 U L-254 M M-30 M I N$. M (Se quence Me thod)

Last changed : 5/8/2018 11:00:14 AM bY SYSTEM (modified after loading)

Additional Info : Peak (s) manually integrated
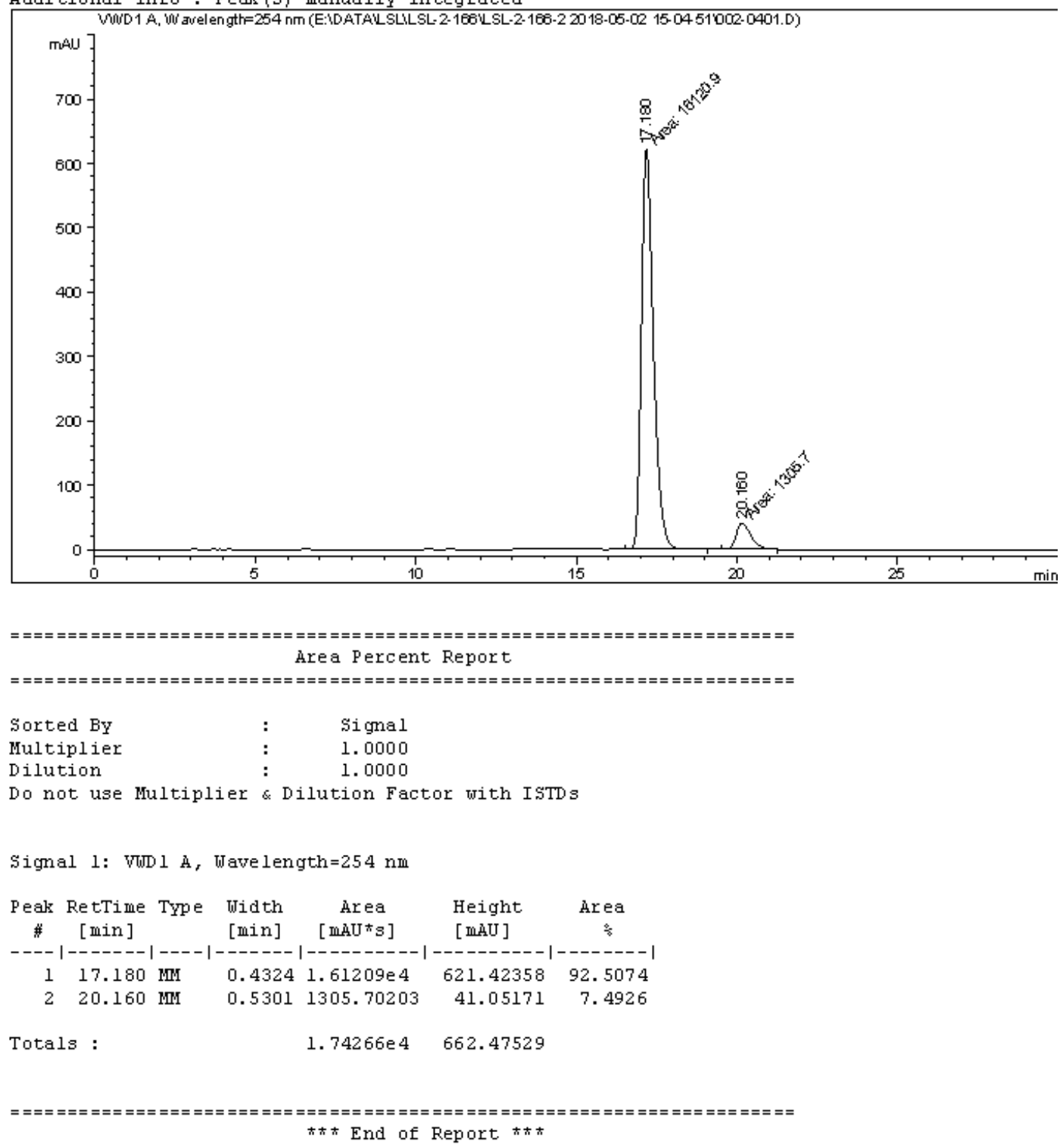
<smiles>COc1ccc([C@H]2COC(=O)C2)cc1</smiles>

Data File E: \DATA \WSW Sample Name: LSL-2-152-1
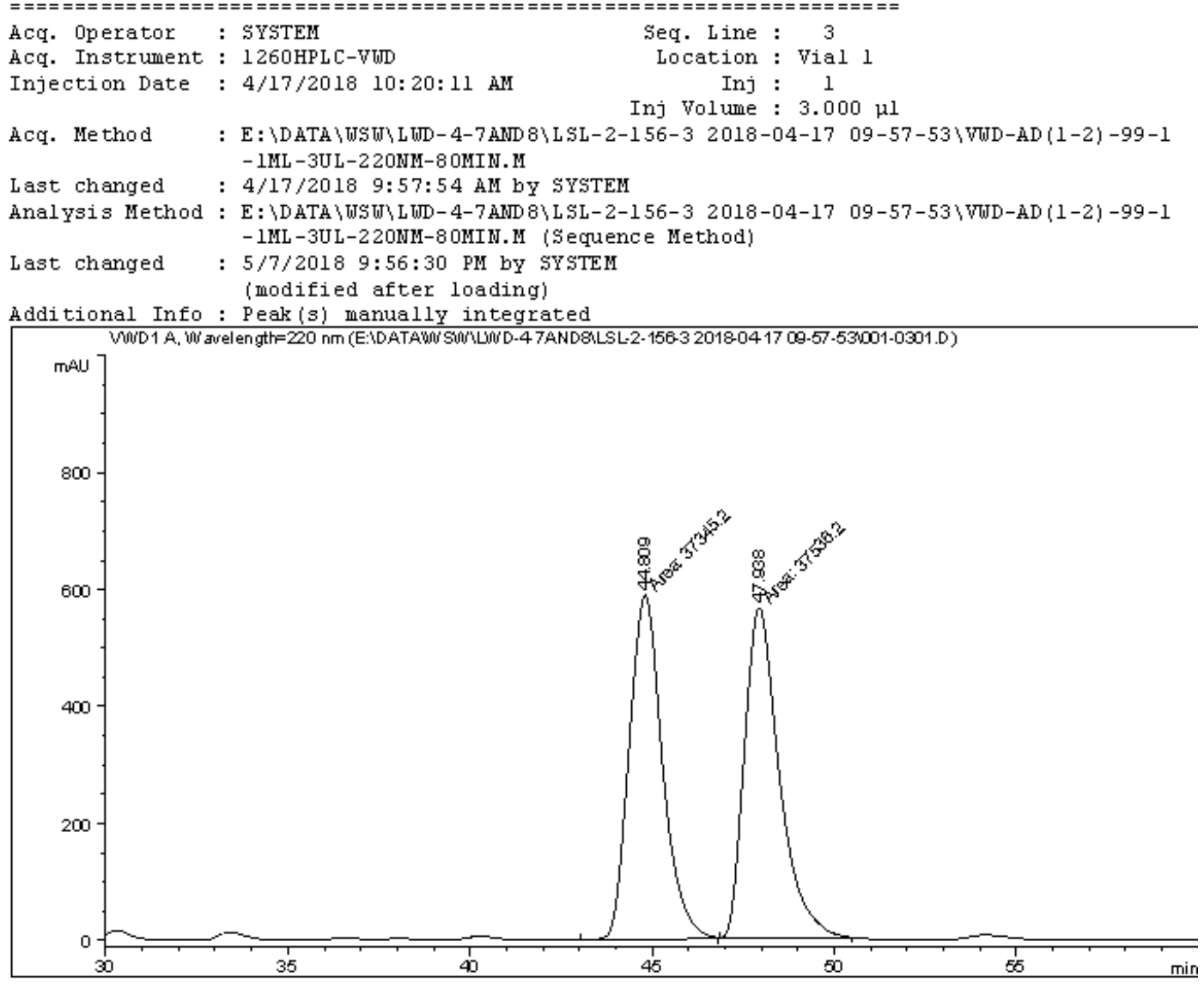

Area Percent Report

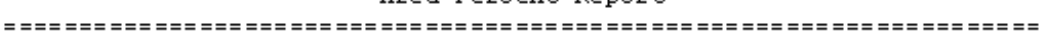

$\begin{array}{lll}\text { Sorted BY } & : & \text { Signal } \\ \text { Multiplier } & : & 1.0000 \\ \text { Dilution } & : & 1.0000\end{array}$

Do not use Multiplier \& Dilution Factor with ISTD s

Signal 1: VWD 1 A, Wave length $=220 \mathrm{~nm}$

\begin{tabular}{|c|c|c|c|c|c|c|}
\hline $\begin{array}{c}\text { Peak } \\
\#\end{array}$ & $\begin{array}{c}\text { Re tTime } \\
\text { [min] }\end{array}$ & Type & $\begin{array}{l}\text { Width } \\
\text { [min] }\end{array}$ & $\begin{array}{c}\text { Area } \\
{\left[\mathrm{mAU}^{*} \mathrm{~s}\right]}\end{array}$ & $\begin{array}{l}\text { Height } \\
\text { [ [MAU] }\end{array}$ & Area \\
\hline 1 & 44.809 & MM & 1.0582 & $3.73452 \mathrm{e} 4$ & 588.18921 & 49.8724 \\
\hline 2 & 47.938 & $\mathrm{MM}$ & 1.1068 & $3.75362 \mathrm{e} 4$ & 565.22797 & 50.1276 \\
\hline ota & & & & 7. 48814 e 4 & 1153.41718 & \\
\hline
\end{tabular}


Data File E: \DATA \WSW\LD-4-7AND $>$ L SL-2-156-3 2018-04-17 09-57-53\002-0401.D Sample Name: LSL-2-156-1

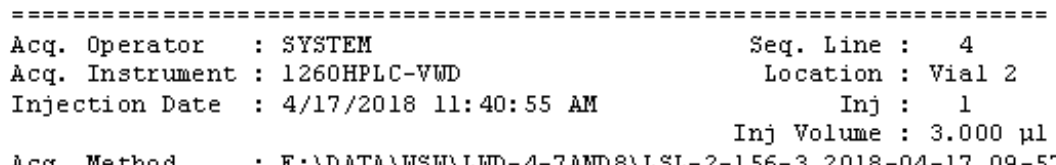

Acq. Me thod : E: \DATA \WSW \LD-4-7AND 8 L SL-2-156-3 2018-04-17 09-57-53 VWD-AD (1-2) -99-1 $-1 M L-3 U L-220 N M-80 M I N . M$

Last changed : 4/17/2018 9:57:54 AM by SYSTEM

Analysis Method : E: \DATA \WSW - IML-3UL-220NM-80MIN.M (Sequence Method)

Last changed : 5/7/2018 10:03:00 PM by SYSTEM

(modified after loading)

Additional Info : Peak (s) manually integrated
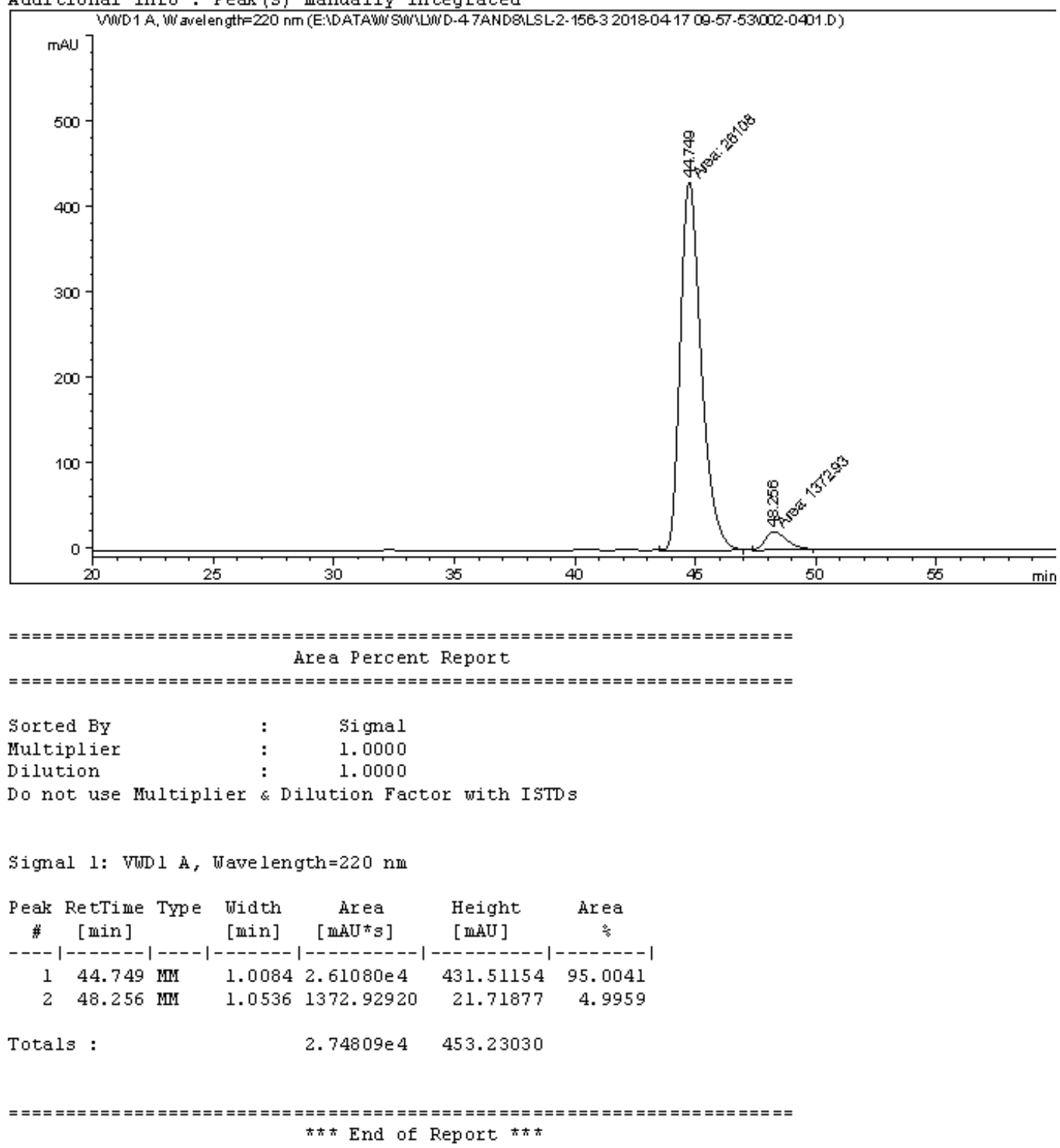
<smiles>O=C1CC(c2ccc(C(F)(F)F)cc2)CO1</smiles>

Data File E: \DATA \LS \LSL-2-173\LSL-2-173-2 2018-05-16 16-53-18\011-1201.D Sample Name: LSL-2-173-1

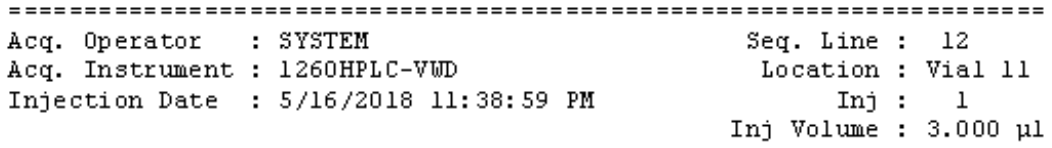

Acq. Me thod : E: \DATA \LS \LSL-2-173\LSL-2-173-2 2018-05-16 16-53-18\VWD-AD(1-2)-99-1$1 \mathrm{ML}-3 \mathrm{UL}-220 \mathrm{MM}-60 \mathrm{MIN}$. M

Last changed : $5 / 16 / 2018$ 8:38:11 PM by SYSTEM

Analysis Method : E: \DATA \LS \LSL-2-173\LSL-2-173-2 2018-05-16 16-53-18\VWD-AD(1-2)-99-11ML-3UL-220NM-60MIN.M (Sequence Me thod)

Last changed : 5/18/2018 7:45:13 PM bY SYSTEM

(modified after loading)

Additional Info : Peak (s) manually integrated

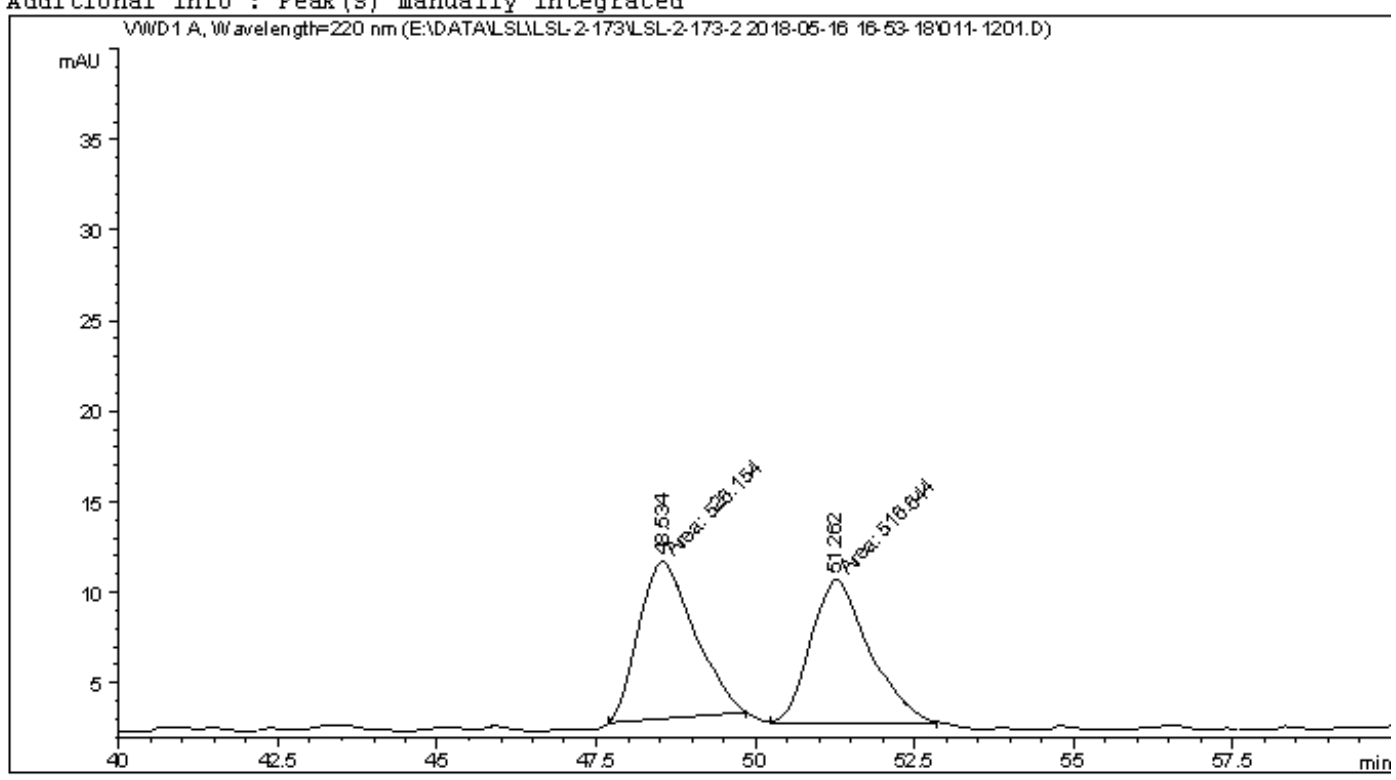

Area Percent Report

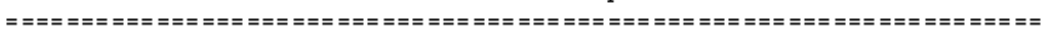

$\begin{array}{lll}\text { Sorted BY } & : & \text { Signal } \\ \text { Multiplier } & : & 1.0000 \\ \text { Dilution } & : & 1.0000\end{array}$

Dilution : $\quad 1.0000$

Do not use Multiplier \& Dilution Factor with ISTD s

Signal 1: VWD 1 A, Wave length $=220 \mathrm{~nm}$

\begin{tabular}{|c|c|c|c|c|c|c|}
\hline $\begin{array}{c}\text { Peak } \\
\#\end{array}$ & $\begin{array}{c}\text { RetTime } \\
\text { [min] }\end{array}$ & Type & $\begin{array}{l}\text { Width } \\
\text { [min] }\end{array}$ & $\begin{array}{c}\text { Area } \\
\left.\text { [ } \mathrm{m} \mathrm{AU}^{*} \mathrm{~s}\right]\end{array}$ & $\begin{array}{l}\text { Height } \\
\text { [ mAU] }\end{array}$ & $\begin{array}{c}\text { Area } \\
\stackrel{4}{*}\end{array}$ \\
\hline 1 & 48.534 & MM & 1.0090 & 526.15405 & 8.69124 & 50.4560 \\
\hline 2 & 51.262 & $\mathrm{MM}$ & 1.0745 & 516.64374 & 8.01378 & 49.5440 \\
\hline
\end{tabular}

Totals: $\quad 1042.79779 \quad 16.70502$ 
Data File E: \DATA \LS \LSL-2-173\LSL-2-173-2 2018-05-16 16-53-18\012-1301.D Sample Name: LSL-2-174-1

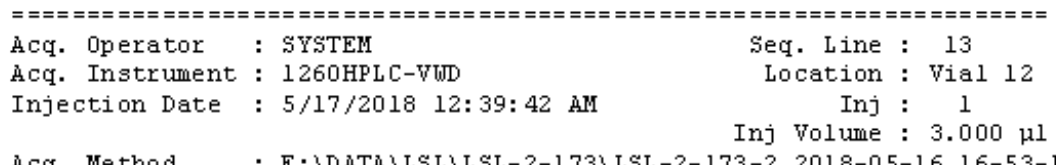

Acq. Me thod : E: \DATA \LSL \LSL-2-173\LSL-2-173-2 2018-05-16 16-53-18\VWD-AD(1-2)-99-11ML-3UL-22 OMM-60MIN. M

Last changed : 5/16/2018 8:38:11 PM by SYSTEM

Analysis Method : E: \DATA \LSL \SL-2-173\LSL-2-173-2 2018-05-16 16-53-18\VWD-AD(1-2)-99-11ML-3UL-22 OMM-60MIN.M (Sequence Me thod)

Last changed : 6/14/2018 7:36:59 PM by SYSTEM

(modified after loading)

Additional Info : Peak (s) manually integrated
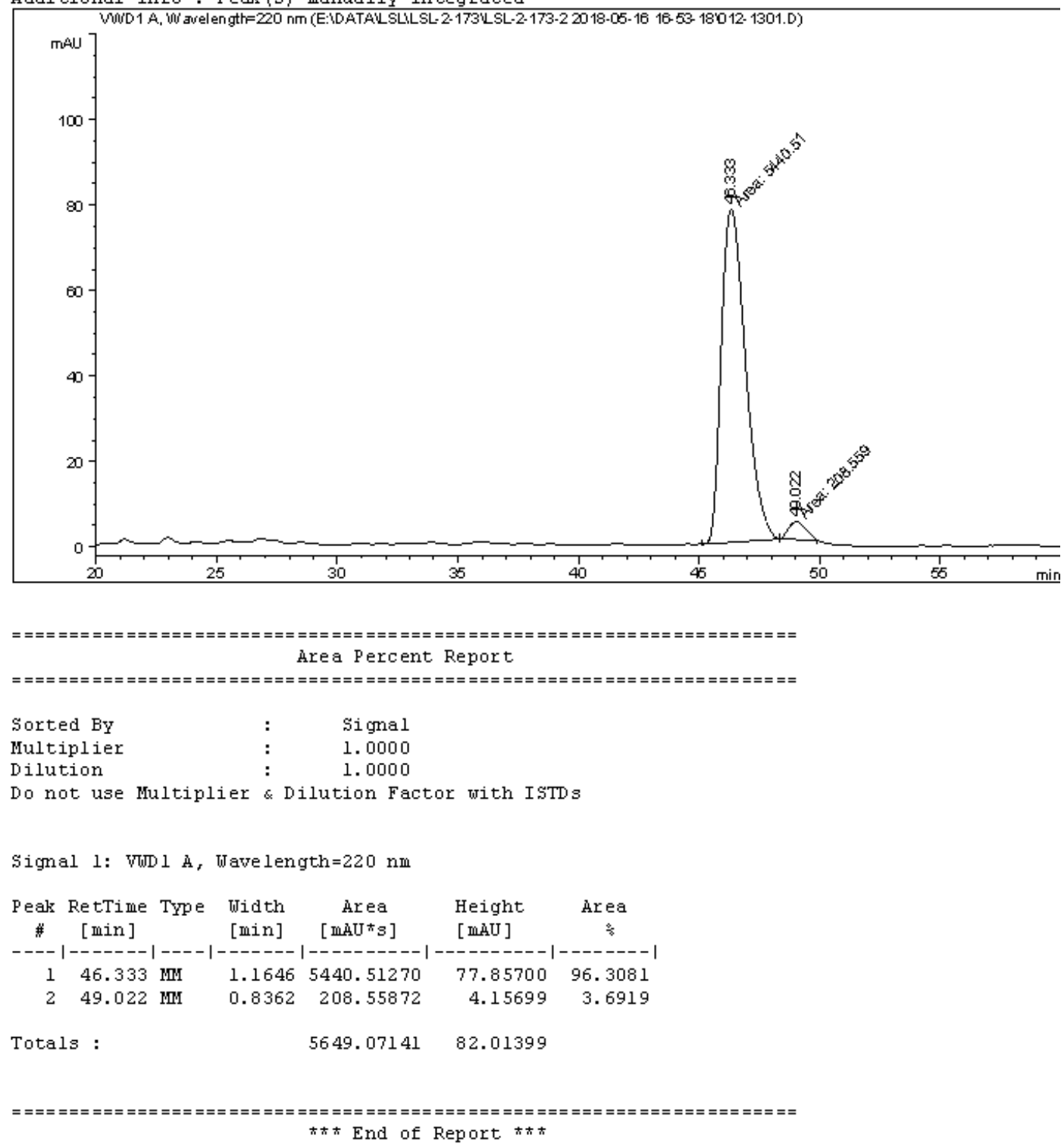
<smiles>CCOC(=O)c1ccc(C2COC(=O)C2)cc1</smiles>

Data File E: \ATA \LSL\LL-2-171-2\LSL-2-171-2 2018-05-06 13-13-04\021-0301.D Sample Name: LSL-2-170-2
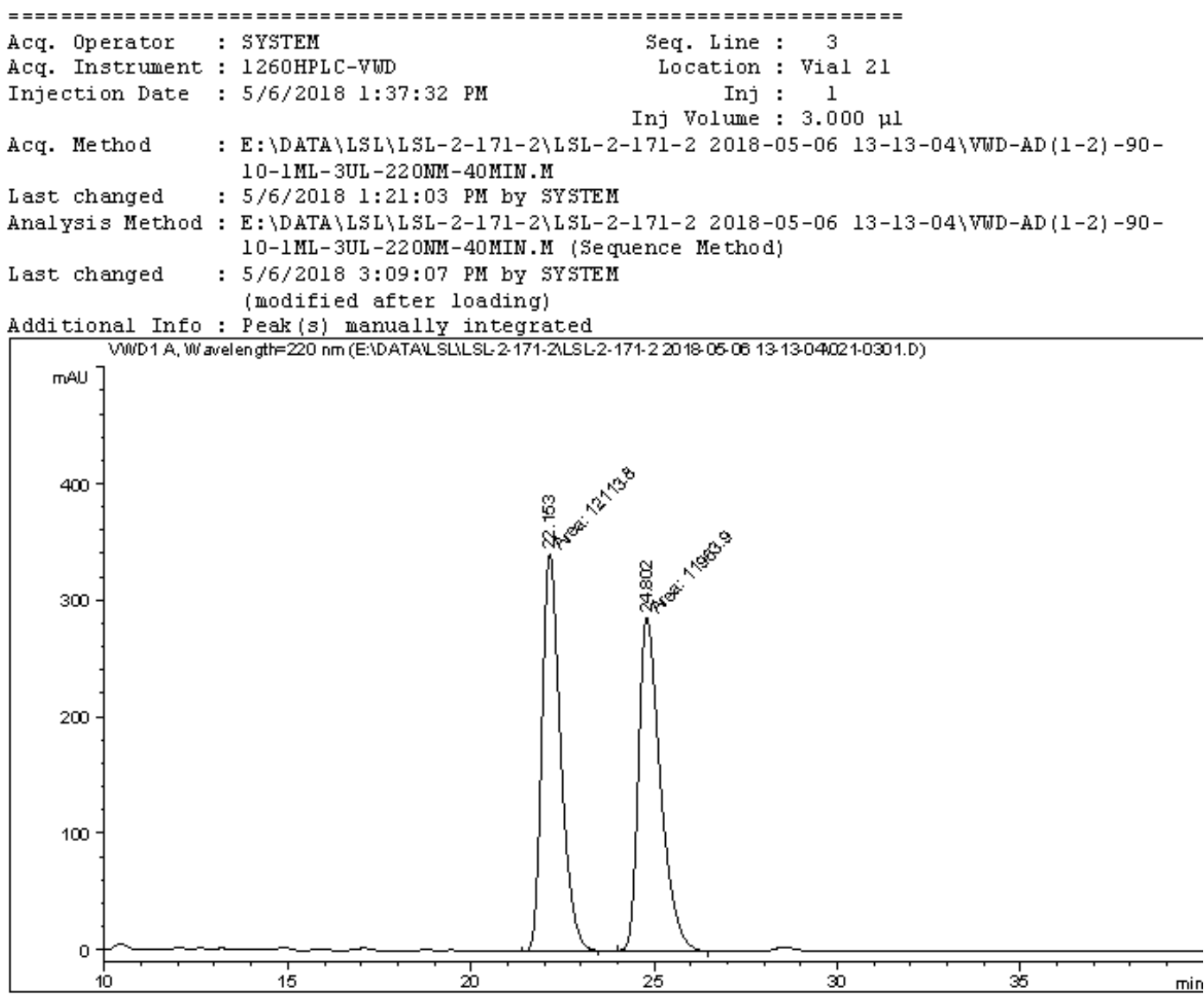

\section{Area Percent Report}

\begin{tabular}{|c|c|}
\hline sorted By & : \\
\hline Multiplier & : \\
\hline Dilution & : \\
\hline
\end{tabular}

Do not use Multiplier \& Dilution Factor with ISTDs

Signal 1: VWD 1 A, Wave length $=220 \mathrm{~nm}$

\begin{tabular}{|c|c|c|c|c|c|c|}
\hline $\begin{array}{c}\text { Peak } \\
\#\end{array}$ & $\begin{array}{c}\text { Re tTime } \\
\text { [min] }\end{array}$ & Type & $\begin{array}{l}\text { Width } \\
\text { [min] }\end{array}$ & $\begin{array}{c}\text { Area } \\
{\left[\mathrm{MAU} U^{*} s\right]}\end{array}$ & $\begin{array}{l}\text { Height } \\
\text { [ mAU] }\end{array}$ & $\begin{array}{c}\text { Area } \\
\stackrel{4}{*}\end{array}$ \\
\hline 1 & 22.153 & $\mathrm{MM}$ & 0.5909 & $1.21138=4$ & 341,65842 & 50,3112 \\
\hline 2 & 24.802 & $\mathrm{MM}$ & 0.6969 & 1. $19639 \mathrm{e} 4$ & 286.14108 & 49.6888 \\
\hline to & & & & 2. $40777 \mathrm{e} 4$ & 950 & \\
\hline
\end{tabular}


Data File E: \ATA \LSL\LL-2-171-2\LSL-2-171-2 2018-05-06 13-13-04\022-0401.D Sample Name: L SL-2-171-2

\begin{tabular}{|c|c|c|c|}
\hline Acq. Operator & : SYSTEM & Seq. Line & : \\
\hline Acq. Instrument & : $1260 \mathrm{HPLC}-\mathrm{VWD}$ & Location & : Vial 22 \\
\hline Injection Date & : 5/6/2018 2:18:16 PM & $\begin{array}{r}\text { Inj } \\
\text { Inj Volume }\end{array}$ & $\begin{array}{lc}: & 1 \\
: & 3.000 \mu l\end{array}$ \\
\hline
\end{tabular}

Acq. Me thod : E: \DATA \LSL \LSL-2-171-2\LSL-2-171-2 2018-05-06 13-13-04\VWD-AD (1-2)-9010-1ML-3UL-220MM-40MIN . M

Last changed : 5/6/2018 $1: 21: 03$ PM by SYSTEM

Analysis Method : E: \DATA \LSL \LSL-2-171-2\LSL-2-171-2 2018-05-06 13-13-04\VWD-AD (1-2) -9010-1ML-3UL-22 0MM-40MIN.M (Sequence Me thod)

Last changed : 5/6/2018 3:12:09 PM by SY STEM

(modified after loading)

Additional Info : Peak (s) manually integrated
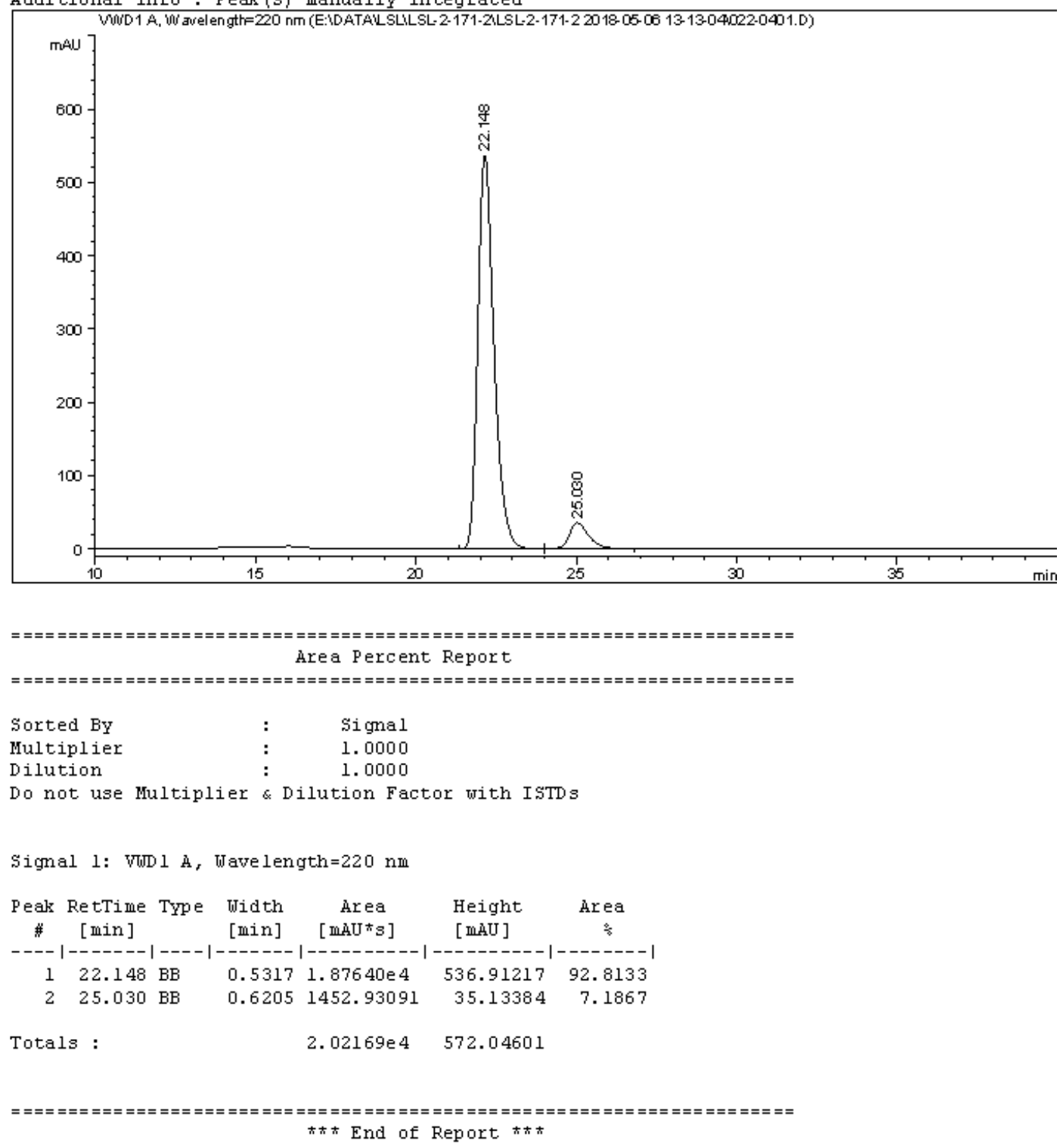
<smiles>O=C1C[C@H](c2ccc(F)cc2)CO1</smiles>

Data File E : \DATA \WSW Sample Name: LSL-2-152-3

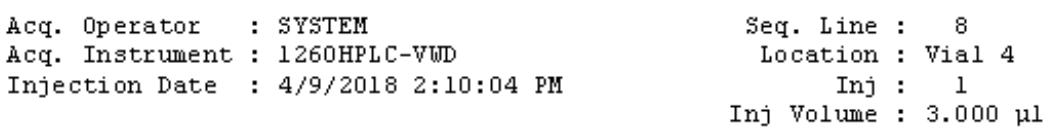

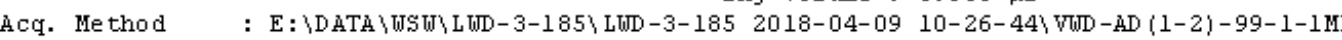
$-3 \mathrm{UL}-22$ ONM $-60 \mathrm{MIN}$. M

Last changed : 4/9/2018 11:50:21 AM by SYSTEM

Analysis Method : E: \DATA \WSW\LWD-3-185 LWD-3-185 2018-04-09 10-26-44\VWD-AD (1-2)-99-1-1ML -3 UL -22 OMM-60MIN.M (Sequence Me thod)

Last changed : 4/30/2018 11:05:41 AM by 5YsTEM

(modified after loading)

Additional Info : Peak ( $s$ ) manually integrated

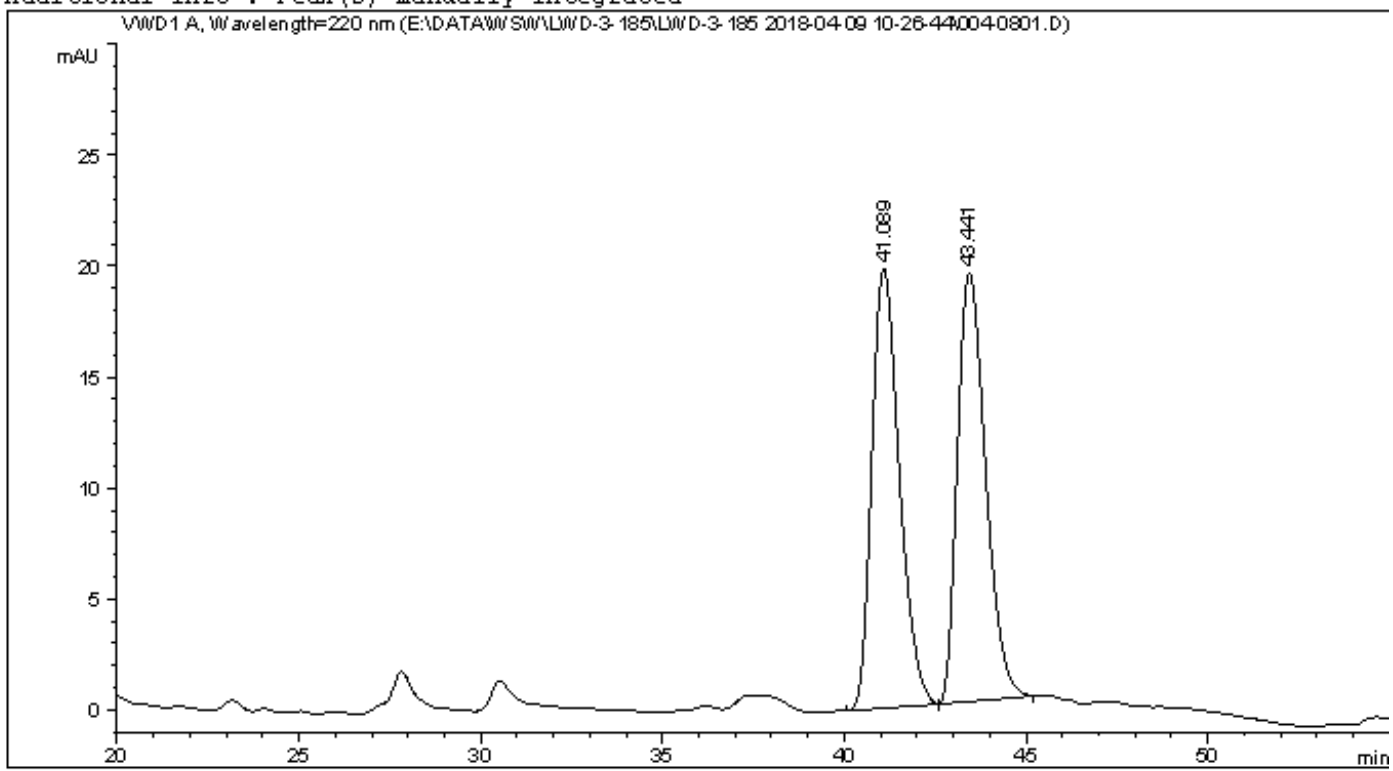

Area Percent Report

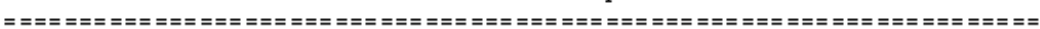

$\begin{array}{lll}\text { Sorted By } & : & \text { Signal } \\ \text { Multiplier } & : & 1.0000 \\ \text { Dilution } & : & 1.0000\end{array}$

Dilution : 1.0000

Do not use Multiplier \& Dilution Factor with ISTD s

Signal 1: VWD $1 \mathrm{~A}$, Wave length $=220 \mathrm{~nm}$

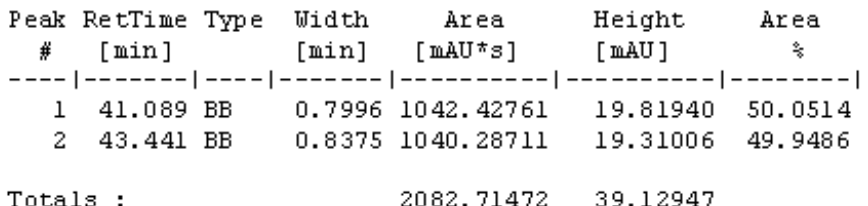

Totals :

$2082.71472 \quad 39.12947$

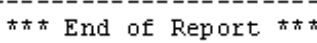


Data File E: \DATA $\backslash$ LS \LSL-2-156\LSL-2-156 2018-04-14 21-53-52\013-0401.D Sample Name: LSL-2-156-3

\begin{tabular}{|c|c|c|c|}
\hline Acq. Operator & : SYSTEM & Seq. Line : & 4 \\
\hline Injection Date & : 4/14/2018 11: 16:55 PM & $\begin{array}{r}\text { Inj } \\
\text { Inj Volume }\end{array}$ & $\frac{1}{3.000 \mu}$ \\
\hline
\end{tabular}

Acq. Me thod : E: \DATA \LSL \LSL-2-156\LSL-2-156 2018-04-14 21-53-52 VWD-AD (1-2)-99-1-1ML $-3 \mathrm{UL}-22$ OMM-60MIN.M

Last changed : 4/14/2018 9:53:53 PM by SYSTEM

Analysis Method : E: \DATA \LSL \LL-2-156\LSL-2-156 2018-04-14 21-53-52 VWD-AD (1-2)-99-1-1ML -3UL-22 ONM-60MIN.M (Sequence Me thod)

Last changed : 4/30/2018 11:21:11 AM by SYSTEM

(modified after loading)

Additional Info : Peak (s) manually integrated
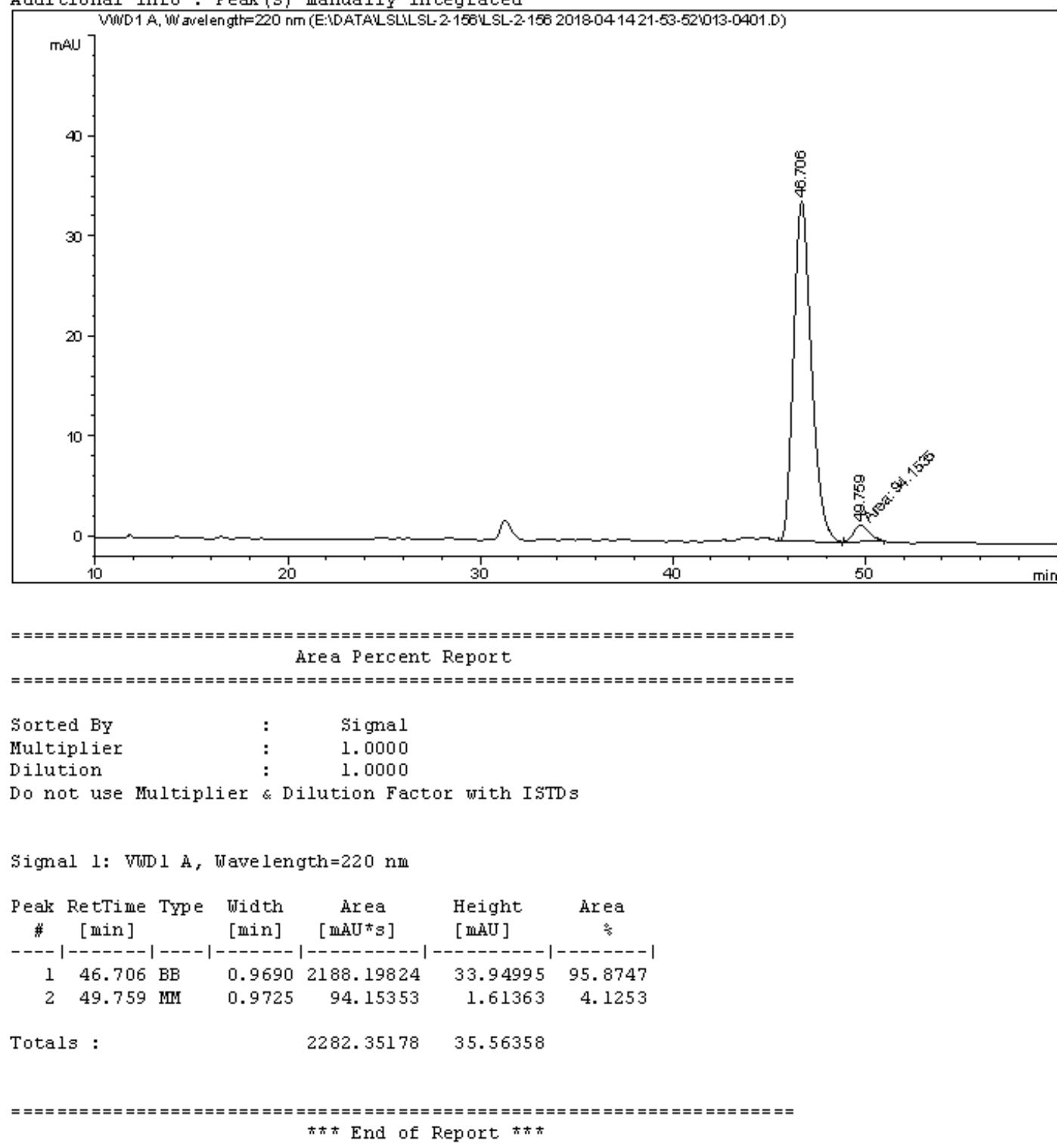
<smiles>O=C1CC(c2ccc(Cl)cc2)CO1</smiles>

Data File E: \DATA \YJX\YJX-1-124-0504\YJX-1-124 2018-05-04 16-21-44\021-1801.D Sample Name: LSL-2-170-1

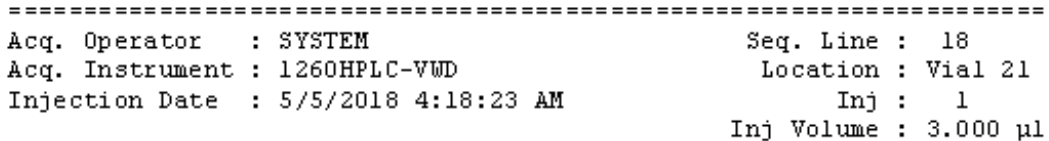

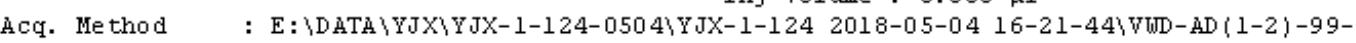
$1-1 M L-3 U L-220 M M-60 M I N . M$

Last changed : 5/4/2018 8:59:53 PM by SYSTEM

Analysis Method : E: \DATA \YJX\YJX-1-124-0504\YJX-1-124 2018-05-04 16-21-44\VWD-AD(1-2)-991-1ML-3UL-220MM-60MIN. M (Sequence Method)

Last changed : 5/5/2018 2:32:12 PM by SY STEM

(modified after loading)

Additional Info : Peak ( $s$ ) manually integrated

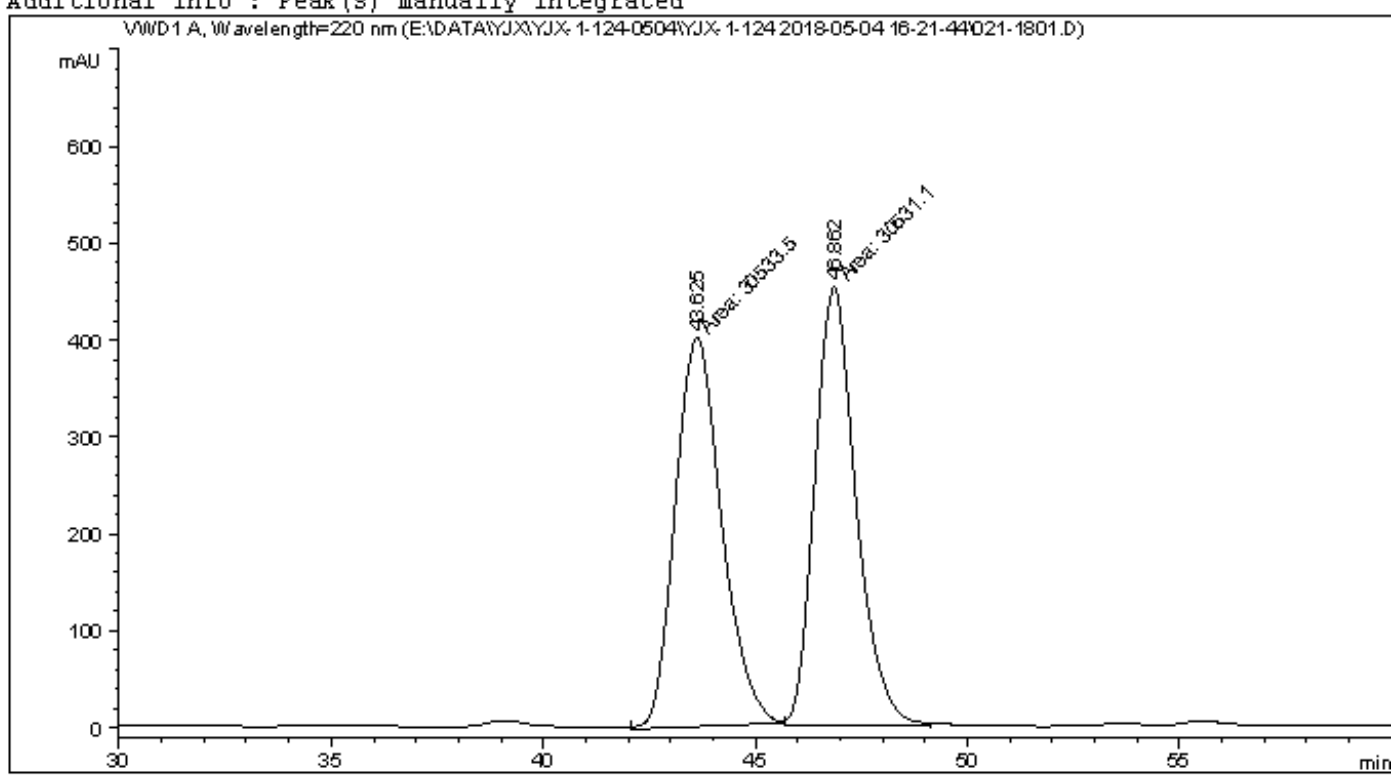

Area Percent Report

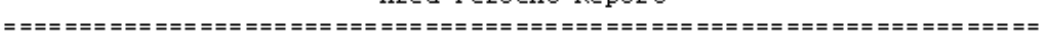

$\begin{array}{lll}\text { Sorted BY } & : & \text { Signal } \\ \text { Multiplier } & : & 1.0000 \\ \text { Dilution } & : & 1.0000\end{array}$

Do not use Multiplier \& Dilution Factor with ISTD s

Signal 1: VWD $1 \mathrm{~A}$, Wave length $=220 \mathrm{~nm}$

\begin{tabular}{|c|c|c|c|c|c|c|}
\hline $\begin{array}{c}\text { Peak } \\
\#\end{array}$ & $\begin{array}{l}\text { Re tTime } \\
\text { [min] }\end{array}$ & Type & $\begin{array}{l}\text { Width } \\
\text { [min] }\end{array}$ & $\begin{array}{c}\text { Area } \\
{\left[\mathrm{mAU} U^{*} s\right]}\end{array}$ & $\begin{array}{l}\text { Height } \\
\text { [ } \mathrm{mAU}]\end{array}$ & $\begin{array}{c}\text { Àrea } \\
\text { s: }\end{array}$ \\
\hline 1 & 43.625 & $\mathrm{MM}$ & 1.2692 & $3.05335 \mathrm{e} 4$ & 400.94 & 50.0020 \\
\hline 2 & 46.862 & $\mathrm{MM}$ & 1.1234 & 3. 053 lle 4 & 452.94302 & 49.9980 \\
\hline otal & & & & $6.10646 \mathrm{e} 4$ & 853.88629 & \\
\hline
\end{tabular}

ネネ゙ End of Report 
Data File E: \DATA \YJXYJX-1-124-0504\YJX-1-124 2018-05-04 16-21-44\022-1901.D Sample Name: LSL-2-171-1

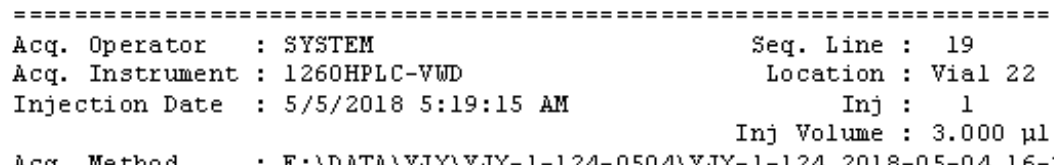

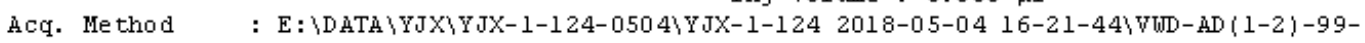
1-1ML-3UL-220MM-60M IN. M

Last changed : 5/4/2018 8:59:53 PM by SYSTEM

Analysis Method : E: \DATA \YJX\YJX-1-124-0504\YJX-1-124 2018-05-04 16-21-44\VWD-AD(1-2)-991-1ML-3UL-220MM-60MIN. M (Sequence Method)

Last changed : 5/5/2018 2:37:40 PM by SYSTEM

(modified after loading)

Additional Info : Peak (s) manually integrated
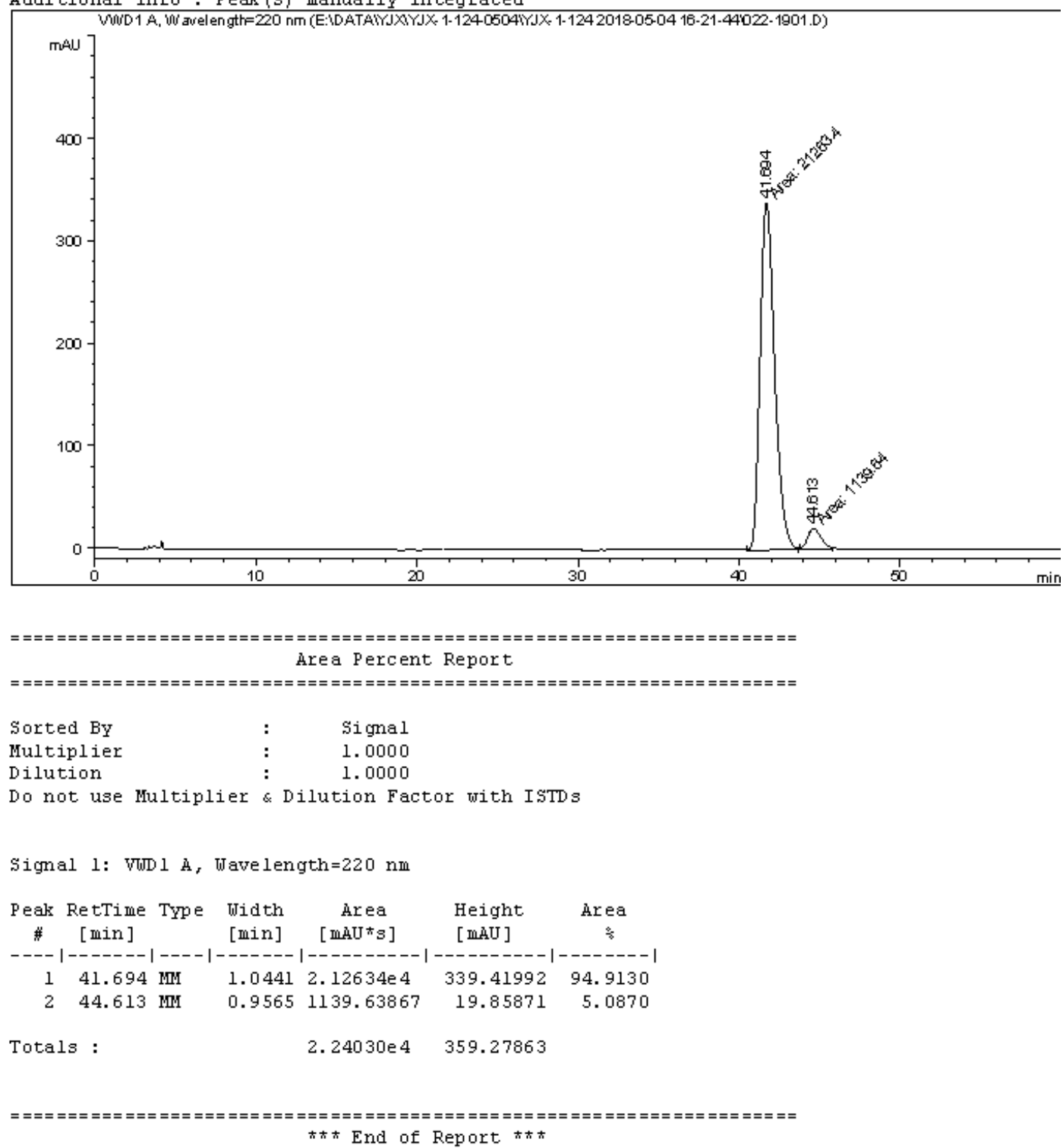
<smiles>O=C1C[C@H](c2cccc(F)c2)CO1</smiles>

$2 \mathrm{k}$

Data File E: \DATA \XZC\AMIN0-ACID XZC-20180409-1 2018-04-09 19-02-51\006-1101.D Sample Name: LSL-2-152-6

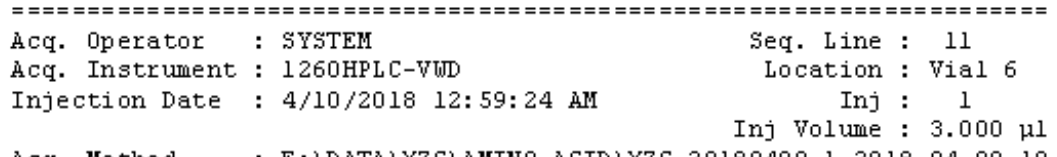

Acq. Me thod : E: \DATA \XZC \AMIN0-ACID XZC-20180409-1 2018-04-09 19-02-51 VID-ADD (1-2)-99 $-1-1$ ML-3UL -220 MM -60 MIN. M

Last changed : 4/9/2018 9:29:40 PM by 5YรTEM

Analysis Method : E: \DATA \XZC\AMIN0-ACID \XZC-20180409-1 2018-04-09 19-02-51 VWD-AD (1-2)-99 -1-1ML-3UL-220MM-60MIN.M (Sequence Me thod)

Last changed : 5/8/2018 10:49:25 AM by SYSTEM

(modified after loading)

Additional Info : Peak (s) manually integrated

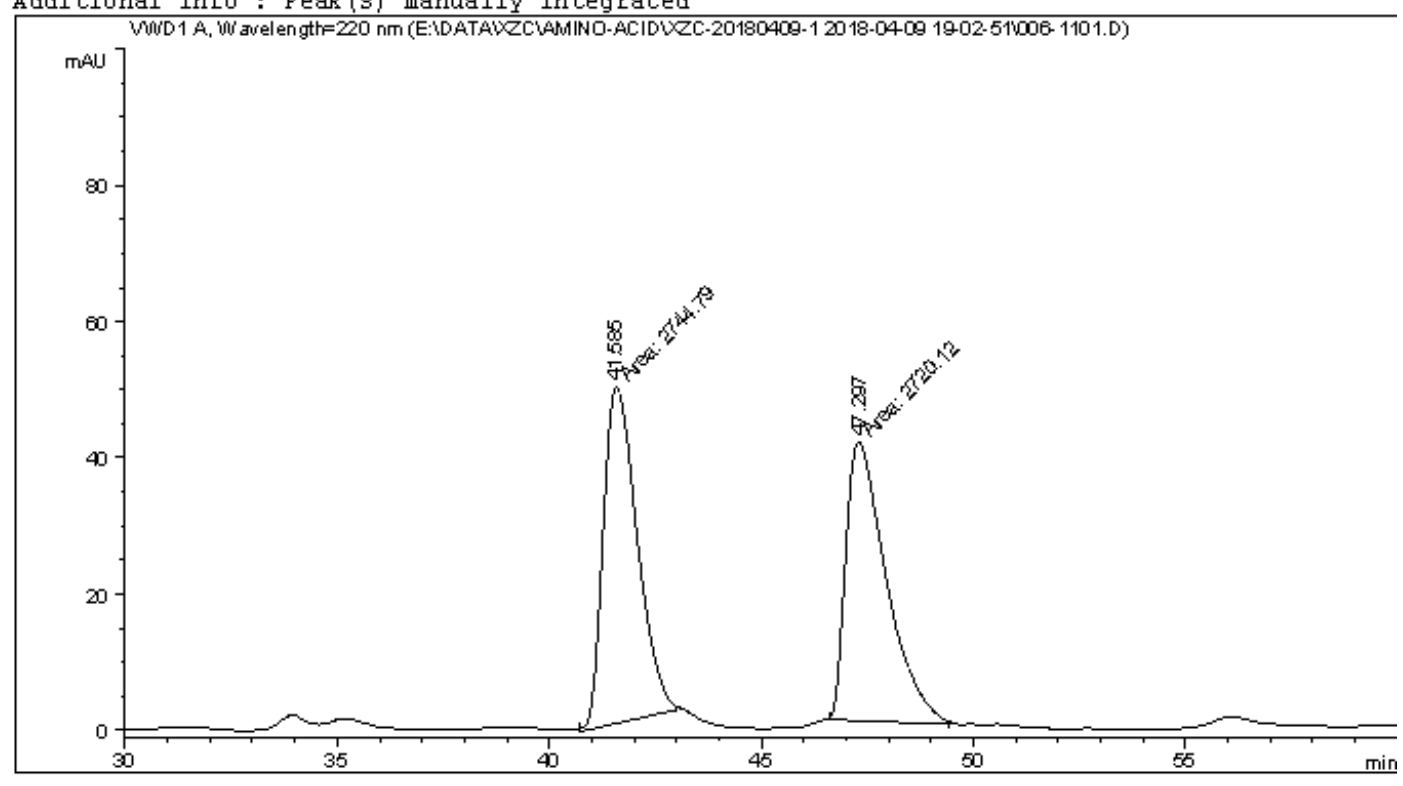

Area Percent Report

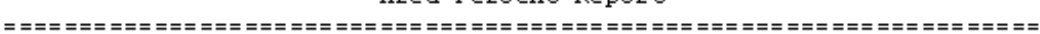

$\begin{array}{lll}\text { Sorted BY } & : & \text { Signal } \\ \text { Multiplier } & : & 1.0000 \\ \text { Dilution } & : & 1.0000\end{array}$

Do not use Multiplier \& Dilution Factor with ISTDs

Signal 1: VWD $1 \mathrm{~A}$, Wave length $=220 \mathrm{~nm}$

\begin{tabular}{|c|c|c|c|c|c|c|}
\hline $\begin{array}{c}\text { Peak } \\
\#\end{array}$ & $\begin{array}{c}\text { Re tTime } \\
\text { [min] }\end{array}$ & Type & $\begin{array}{l}\text { Width } \\
\text { [min] }\end{array}$ & $\begin{array}{c}\text { Area } \\
{\left[\mathrm{mAU}^{*} \mathrm{~s}\right]}\end{array}$ & $\begin{array}{l}\text { Height } \\
\text { [ [MAU] }\end{array}$ & Area \\
\hline 1 & 41.585 & $\mathrm{MM}$ & 0.9256 & 2744.79272 & 49.42210 & 50.2258 \\
\hline 2 & 47.297 & $\mathrm{MM}$ & 1.1072 & 2720.11646 & 40.94407 & 49.7742 \\
\hline ota & : & & & 5464.90918 & 6617 & \\
\hline
\end{tabular}

ネネ゙ End of Report ネ* 
Data File E: \DATA \LS \LSL-2-156-2\LSL-2-156-2 2018-04-15 12-13-34\016-0301.D Sample Name: L SL-2-156-6

\begin{tabular}{|c|c|c|c|}
\hline Acq. Operator & : SYSTEM & Seq. Line & 3 \\
\hline Acq. Instrument & $: 1260 \mathrm{HPLC}-\mathrm{VWD}$ & Location & : Vial 16 \\
\hline Injection Date & : 4/15/2018 12:38:04 PM & $\begin{array}{r}\text { Inj } \\
\text { Inj Volume }\end{array}$ & $\begin{array}{l}: \quad 1 \\
: \quad 3.000 \mu l\end{array}$ \\
\hline
\end{tabular}

Acq. Me thod : E: \DATA \LSL \LSL-2-156-2\LSL-2-156-2 2018-04-15 12-13-34\VWD-ADD (1-2) -99-1 $-1 M L-3 U L-220 N M-80 M I N . M$

Last changed : 4/15/2018 12:13:34 PM by SYSTEM

Analysis Method : E: \DATA \LSL \SL-2-156-2\LSL-2-156-2 2018-04-15 12-13-34\VWD-AD (1-2) -99-1 - IML-3UL-220NM-80MIN.M (Sequence Method)

Last changed : 5/16/2018 5:03:18 PM by SYSTEM

(modified after loading)

Additional Info : Peak (s) manually integrated
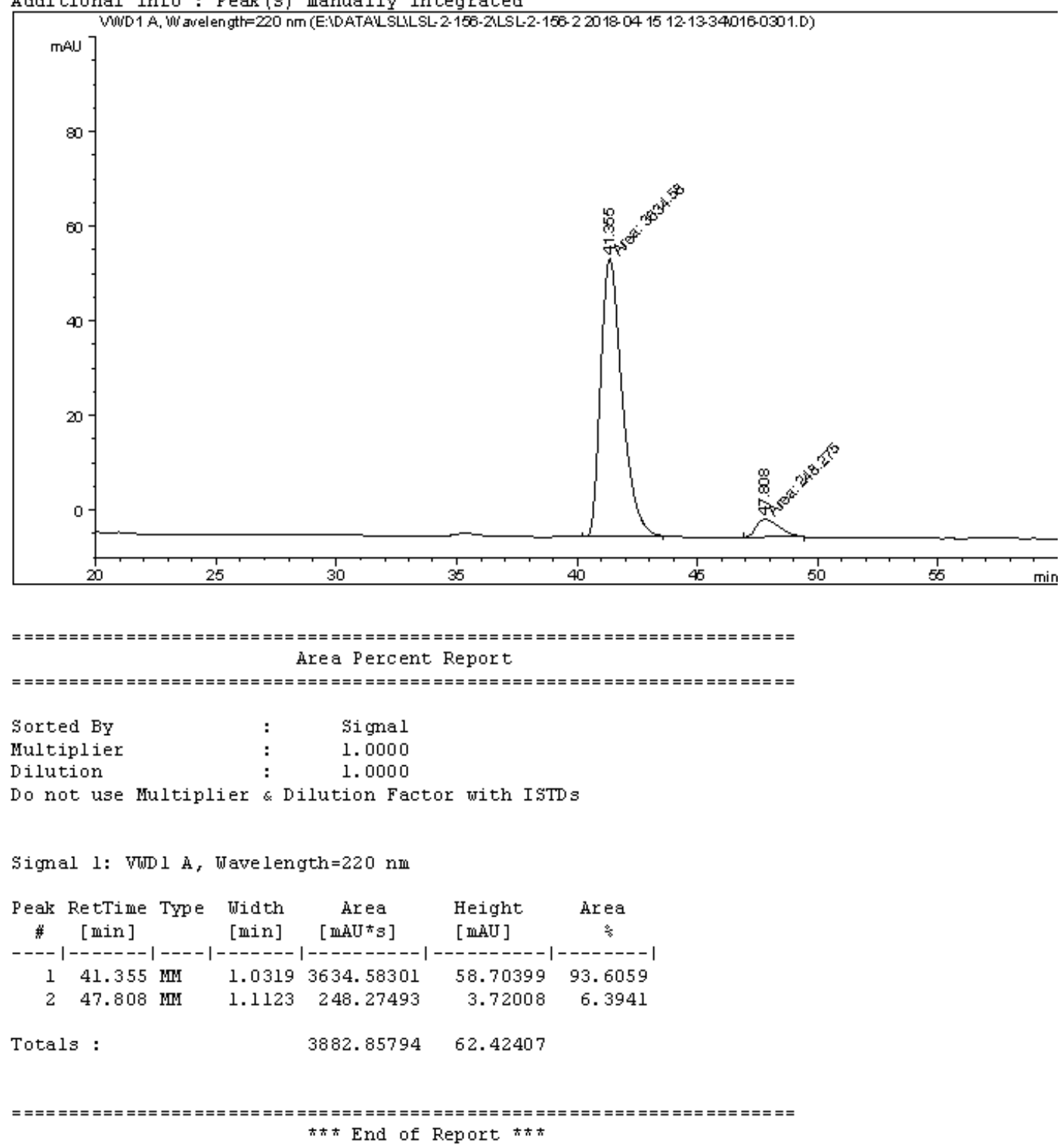
<smiles>Cc1ccccc1[C@H]1COC(=O)C1</smiles>

Data File D: \DATA \CZY \CZY-RAC-1129\CZY-20181129-RAC 2018-11-29 15-21-35\042-0501.D Sample Name: LSL-3-164-3

\begin{tabular}{|c|c|c|}
\hline $\begin{array}{l}\text { Acq. Operator } \\
\text { Acq. Instrument }\end{array}$ & $\begin{array}{l}: \\
: \text { Instrument } 1\end{array}$ & $\begin{aligned} & \text { Seq. Line : } 5 \\
& \text { Location : Vial } 42\end{aligned}$ \\
\hline Injection Date & : 11/29/2018 6:37:05 PM & $\begin{aligned} \text { Inj }: & 1 \\
\text { Inj Volume }: & 3.000\end{aligned}$ \\
\hline
\end{tabular}

Acq. Method : D: \DATA \CZY\CZY-RAC-1129\CZY-20181129-RAC 2018-11-29 15-21-35\VWD-AD(1-2)95-5-1ML-3UL-220NM-40MIN. M

Last changed : 6/7/2018 11:25:33 AM

Analysis Method: D: \METHOD\LYH\VWD-AD(1-2)-95-5-0.5ML-1UL-210NM-70MIN.M

Last changed : 1/7/2019 7:53:07 PM

(modified after loading)

Additional Info: Peak(s) manually integrated

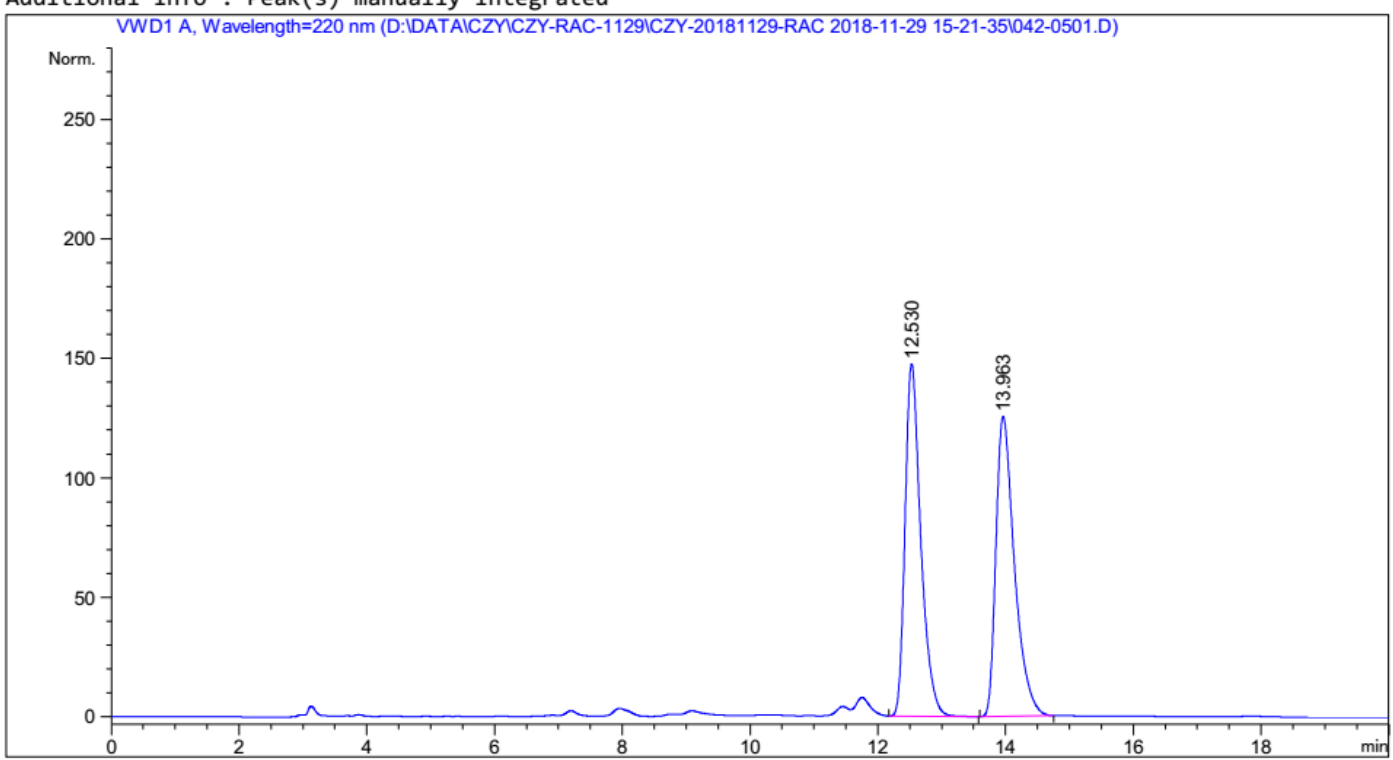

Area Percent Report

\begin{tabular}{|c|c|c|}
\hline Sorted By & : & Signal \\
\hline Multiplier & : & 1.0000 \\
\hline Dilution & : & 1.000 \\
\hline
\end{tabular}

Use Multiplier \& Dilution Factor with ISTDs

Signal 1: VWD1 A, Wavelength $=220 \mathrm{~nm}$

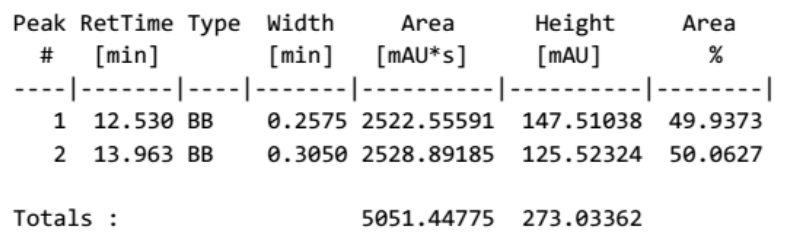


Data File D: \DATA \CZY \CZY-RAC-1129\CZY-20181129-RAC 2018-11-29 15-21-35\043-0601.D Sample Name: LSL-3-174-1

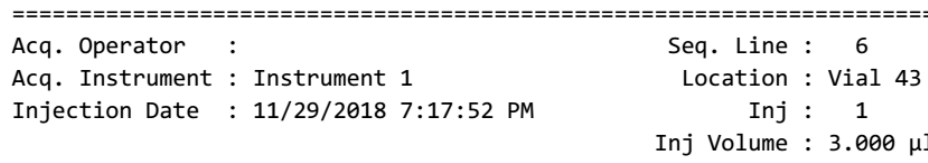

Acq. Method : D: \DATA \CZY \CZY-RAC-1129\CZY-20181129-RAC 2018-11-29 15-21-35\VWD-AD(1-2)95-5-1ML-3UL-220NM-40MIN.M

Last changed : 6/7/2018 11:25:33 AM

Analysis Method: D: \METHOD\LYH\VWD-AD(1-2)-95-5-0.5ML-1UL-210NM-70MIN.M

Last changed : 1/7/2019 7:55:08 PM (modified after loading)

Additional Info: Peak(s) manually integrated

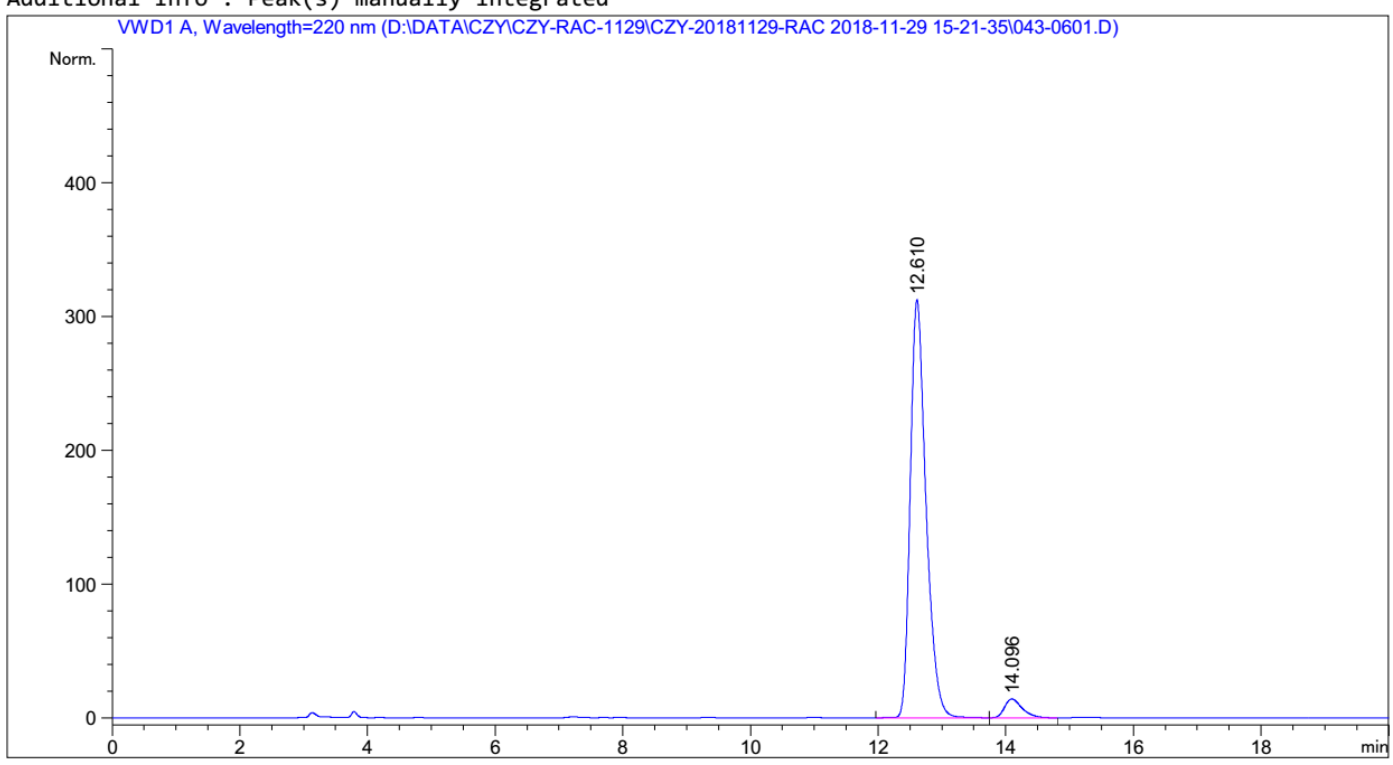

Area Percent Report

$\begin{array}{lll}\text { Sorted By } & : & \text { Signal } \\ \text { Multiplier } & : & 1.0000 \\ \text { Dilution } & : & 1.0000\end{array}$

Use Multiplier \& Dilution Factor with ISTDs

Signal 1: VWD1 A, Wavelength $=220 \mathrm{~nm}$

\begin{tabular}{|c|c|c|c|c|c|}
\hline $\begin{array}{c}\text { Peak } \\
\#\end{array}$ & $\begin{array}{l}\text { RetTime Type } \\
\text { [min] }\end{array}$ & $\begin{array}{l}\text { Width } \\
\text { [min] }\end{array}$ & $\begin{array}{c}\text { Area } \\
{\left[\mathrm{mAU}^{*} \mathrm{~s}\right]}\end{array}$ & $\begin{array}{l}\text { Height } \\
\text { [mAU] }\end{array}$ & $\begin{array}{c}\text { Area } \\
\%\end{array}$ \\
\hline & & & $1--2-0-0-1$ & 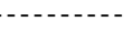 & - \\
\hline 1 & $12.610 \mathrm{BB}$ & 0.2591 & 5355.99219 & 312.23267 & 95.1798 \\
\hline 2 & 14.096 ВВ & 0.2910 & 271.24554 & 14.06377 & 4.8202 \\
\hline Tota & : & & 5627.23773 & 326.29644 & \\
\hline
\end{tabular}


<smiles>Cc1ccc([C@H]2COC(=O)C2)cc1C</smiles>

Data File E: \DATA \LL \LSL-2-173\LSL-2-173-2 2018-05-16 16-53-18\013-1001.D Sample Name: LSL-2-173-2

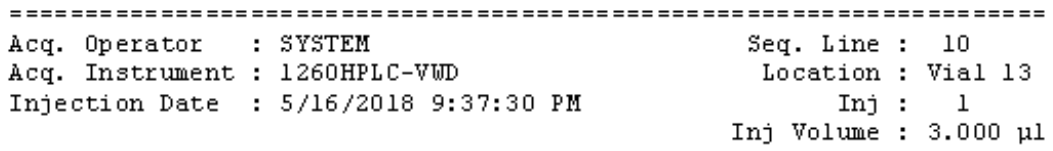

Acq. Me thod : E: \DATA \LS \LSL-2-173\LSL-2-173-2 2018-05-16 16-53-18\VWD-AD(1-2)-99-1$1 \mathrm{ML}-3 \mathrm{UL}-220 \mathrm{MM}-60 \mathrm{MIN}$. M

Last changed : $5 / 16 / 2018$ 8:38:11 PM by SYSTEM

Analysis Method : E: \DATA \LSL \LL-2-173\LSL-2-173-2 2018-05-16 16-53-18\VID-AD (1-2)-99-11ML-3UL-220NM-60MIN.M (Sequence Me thod)

Last changed : 6/14/2018 7:23:27 PM bY SYSTEM

(modified after loading)

Additional Info : Peak ( $s$ ) manually integrated

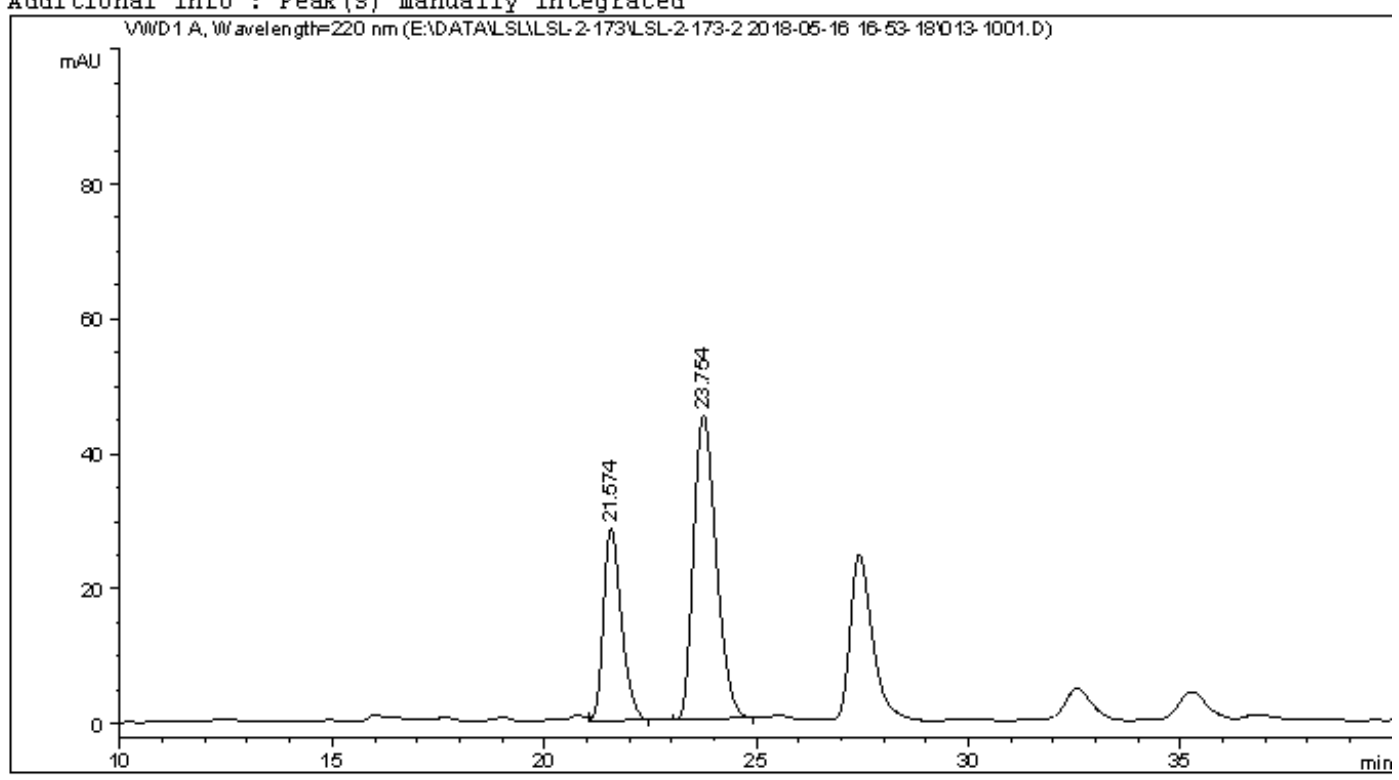

Area Percent Report

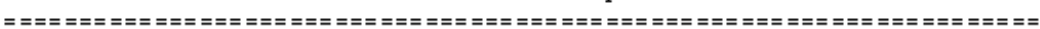

$\begin{array}{lll}\text { Sorted BY } & : & \text { Signal } \\ \text { Multiplier } & : & 1.0000 \\ \text { Dilution } & : & 1.0000\end{array}$

Dilution : 1.0000

Do not use Multiplier \& Dilution Factor with ISTD s

Signal 1: VWD 1 A, Wave length $=220 \mathrm{~nm}$

\begin{tabular}{|c|c|c|c|c|c|c|}
\hline $\begin{array}{c}\text { Peak } \\
\#\end{array}$ & $\begin{array}{c}\text { RetTime } \\
\text { [min] }\end{array}$ & Type & $\begin{array}{l}\text { Width } \\
\text { [min] }\end{array}$ & $\begin{array}{c}\text { Area } \\
{[\text { mand }}\end{array}$ & $\begin{array}{l}\text { Height } \\
\text { [ maU] }\end{array}$ & $\begin{array}{c}\text { Ărea } \\
\stackrel{4}{*}\end{array}$ \\
\hline 1 & 21.574 & VB & 0.4503 & 836.50787 & 28.42912 & 33.3762 \\
\hline 2 & 23.754 & $\mathrm{BB}$ & 0.5811 & 1669.79614 & 44.97640 & 66.6238 \\
\hline otal al a & & & & 2506.30402 & 73.40552 & \\
\hline
\end{tabular}

ネネ* End of Report *ネ* 
Data File E: \DATA \LS \LSL-2-173\LSL-2-173-2 2018-05-16 16-53-18\014-1101.D Sample Name: L SL-2-174-2

\begin{tabular}{|c|c|c|c|}
\hline Acq. Operator & : SYSTEM & seq. Line & : 11 \\
\hline Acq. Instrument & : 1260HPLC-VWD & Location & : Vial 14 \\
\hline Injection Date & 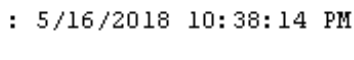 & $\begin{array}{r}\text { Inj } \\
\text { Inj Volume }\end{array}$ & $\begin{array}{l}: \quad 1 \\
: \quad 3.000 \mu .\end{array}$ \\
\hline
\end{tabular}

Acq. Me thod : E: \DATA \LSL \LSL-2-173\LSL-2-173-2 2018-05-16 16-53-18\VWD-AD(1-2)-99-11ML-3UL-220MM-60MIN. M

Last changed : 5/16/2018 8:38:11 PM by SYSTEM

Analysis Method : E: \DATA \LSL \SL-2-173\LSL-2-173-2 2018-05-16 16-53-18\VWD-AD (1-2)-99-11ML-3UL-220MM-60MIN.M (Sequence Me thod)

Last changed : 6/14/2018 7:27:37 PM by SYSTEM

(modified after loading)

Additional Info : Peak (s) manually integrated

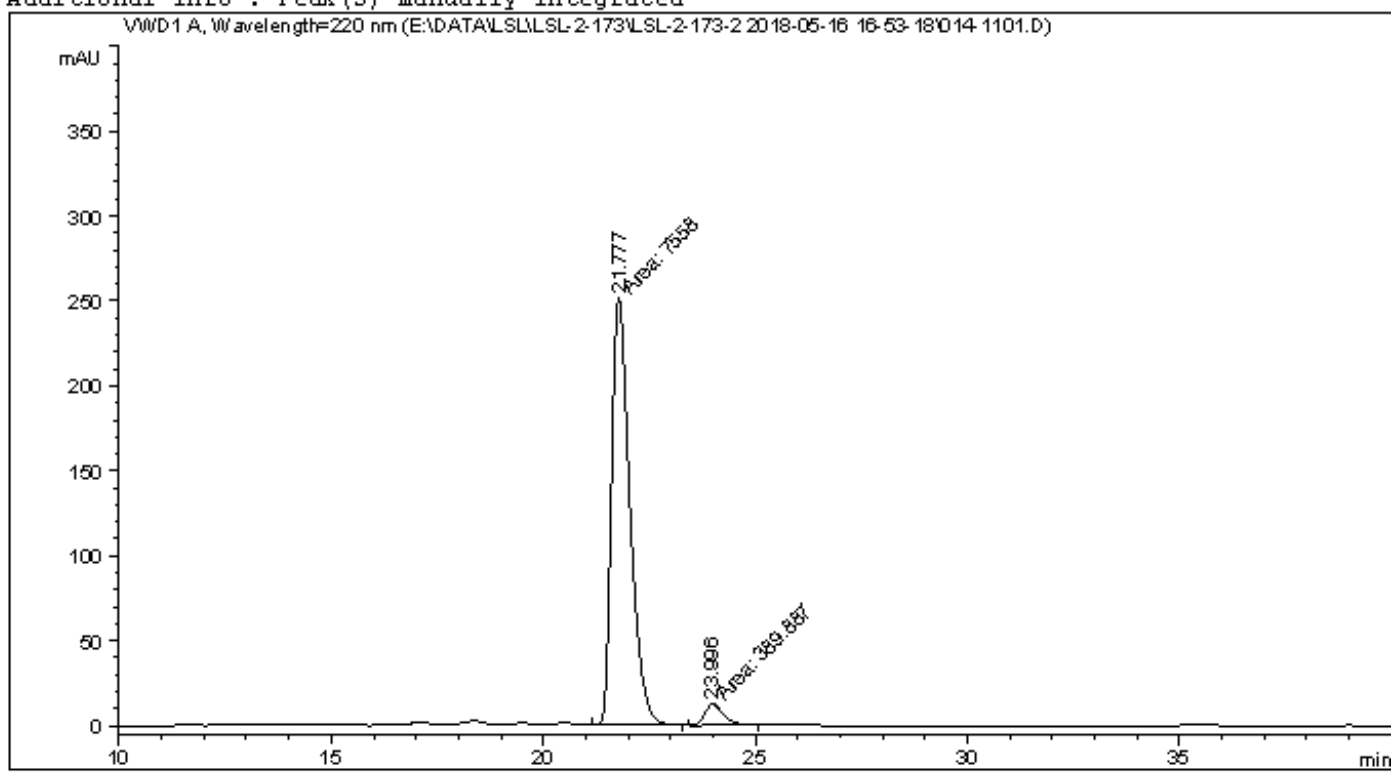

\begin{tabular}{|c|c|c|c|c|}
\hline Sorted By & : & \multicolumn{3}{|c|}{ signal } \\
\hline Multiplier & : & \multicolumn{3}{|l|}{1.0000} \\
\hline Dilution & : & \multicolumn{3}{|c|}{1.0000} \\
\hline \multicolumn{5}{|c|}{ Do not use Multiplier \& Dilution Factor with IsTDs } \\
\hline \multicolumn{5}{|c|}{ Signal 1: VWD $1 \mathrm{~A}$, Wave length $=220 \mathrm{~nm}$} \\
\hline $\begin{array}{c}\text { Peak RetTime Type } \\
\# \quad[\min ]\end{array}$ & $\begin{array}{l}\text { Width } \\
\text { [min] }\end{array}$ & $\begin{array}{c}\text { Area } \\
{\left[\mathrm{m} A U^{*} \mathrm{~s}\right]}\end{array}$ & $\begin{array}{l}\text { Height } \\
\text { [mAU] }\end{array}$ & $\begin{array}{c}\text { hrea } \\
\stackrel{4}{*}\end{array}$ \\
\hline$----|-------|----\mid$ & -------1 & $\mid----------1$ & ---------1 & --------1 \\
\hline $1 \quad 21.777 \mathrm{MM}$ & 0.5015 & 7557.99902 & 251.19183 & 95.0945 \\
\hline $2 \quad 23.996 \mathrm{MM}$ & 0.5251 & 389.88696 & 12.37470 & 4. 9055 \\
\hline Totals : & & 7947.88599 & 263.56654 & \\
\hline
\end{tabular}


<smiles>COc1ccc([C@H]2COC(=O)C2)cc1OC</smiles>

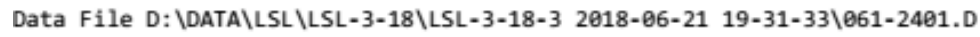
Sample Name: LSL-2-173-3

\begin{tabular}{|c|c|c|}
\hline $\begin{array}{l}\text { Acq. Operator } \\
\text { Acq. Instrument }\end{array}$ & : Instrument 2 & $\begin{array}{l}\text { Seq. Line : } 24 \\
\text { Location : Vial } 61\end{array}$ \\
\hline Injection Date & $: 6 / 22 / 2018 \quad 8: 45: 22$ AM & $\begin{aligned} \text { Inj : } & 1 \\
\text { Inj Volume } & 5.000 \mu \mathrm{l}\end{aligned}$ \\
\hline
\end{tabular}

Acq. Method : D: \DATA ILSLILSL-3-18\LSL-3-18-3 2018-06-21 19-31-33\DAD-0D(1-2)-85-15-1MLSUL-ALL-100MIN.M

Last changed : 6/21/2018 10:10:16 PM

Analysis Method: D: METHOD \HZY \DAD-OD(1-2)-90-10-1ML-2UL-ALL -49MIN.M

Last changed : 9/22/2018 9:49:30 PM (modified after loading)

Additional Info: Peak(s) manually integrated

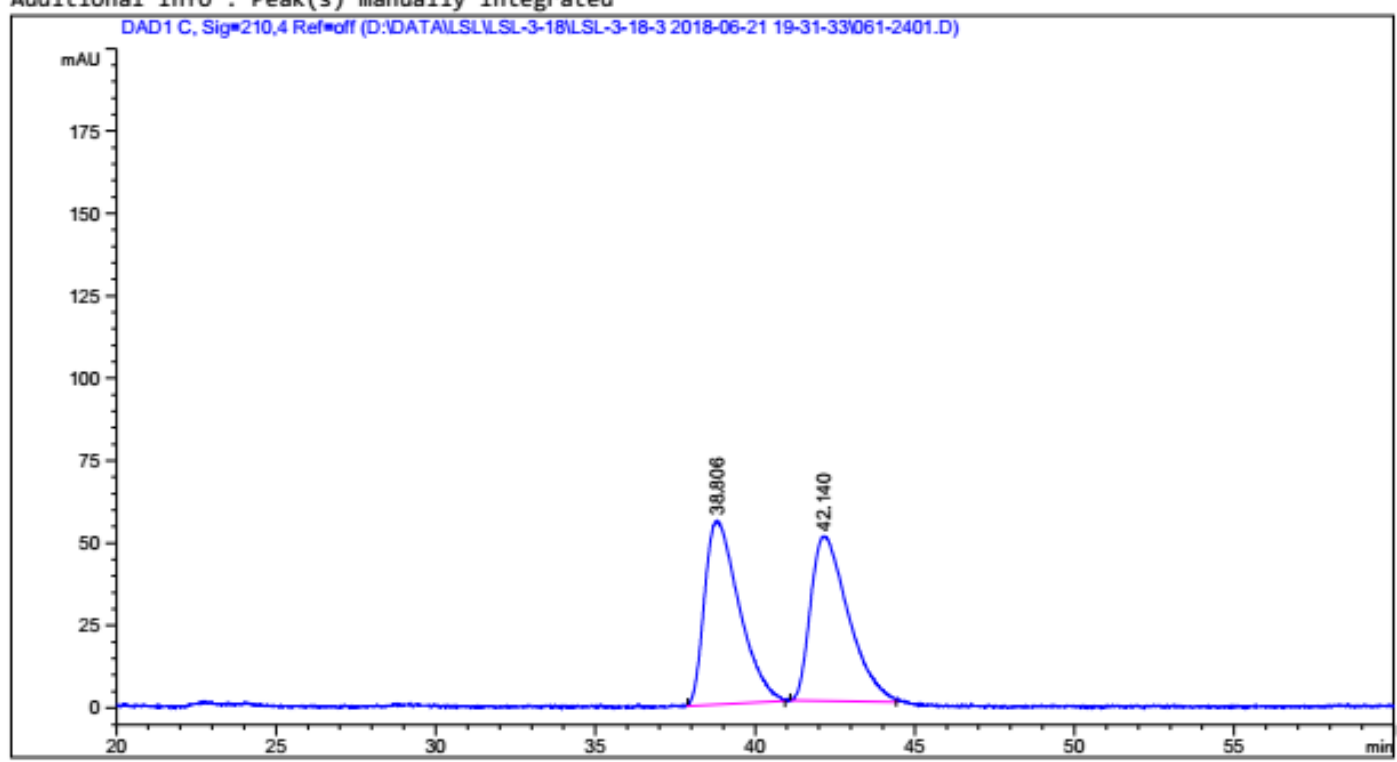

Area Percent Report

$\begin{array}{llr} & & \text { Area Percent } \\ \text { Sorted By } & : & \text { Signal } \\ \text { Multiplier } & : & 1.0000 \\ \text { Dilution } & : & 1.0000\end{array}$

Use Multiplier \& Dilution Factor with ISTDs

Signal 1: DAD1 C, Sig=210,4 Ref=off

\begin{tabular}{|c|c|c|c|c|c|c|}
\hline $\begin{array}{c}\text { Peak } \\
\#\end{array}$ & $\begin{array}{c}\text { RetTime } \\
\text { [min] }\end{array}$ & Type & $\begin{array}{l}\text { Width } \\
\text { [min] }\end{array}$ & $\begin{array}{c}\text { Area } \\
\text { [mAU*s] }\end{array}$ & $\begin{array}{l}\text { Height } \\
\text { [mAU] }\end{array}$ & $\begin{array}{c}\text { Area } \\
\%\end{array}$ \\
\hline & & & & 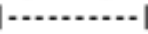 & . & \\
\hline 1 & & & 0.8 & 4210. & 55.87397 & 50.6283 \\
\hline 2 & 42. & BV & 9.9635 & 4106.24756 & 49.92637 & 49.3717 \\
\hline
\end{tabular}

Totals :

$8317.01318 \quad 105.80034$ 
Data File D: \DATA \LSLILSL-3-18\LSL-3-18-3 2018-06-21 19-31-33\062-2501.D Sample Name: 3,4-MeO

Acq. Operator

Acq. Instrument : Instrument 2

Injection Date : 6/22/2018 10:26:21 AM SUL-ALL-100MIN.M

Last changed

(modified after loading)

Seq. Line : 25

Location : Vial 62

Inj : 1

Inj Volume : $5.000 \mu 1$

Analysis Method : D: \METHOD \HZY \DAD-OD(1-2)-90-10-1ML-2UL-ALL-40MIN.M

Last changed : 9/22/2018 10:44:24 PM

(modified after loading)

Additional Info : Peak(s) manually integrated

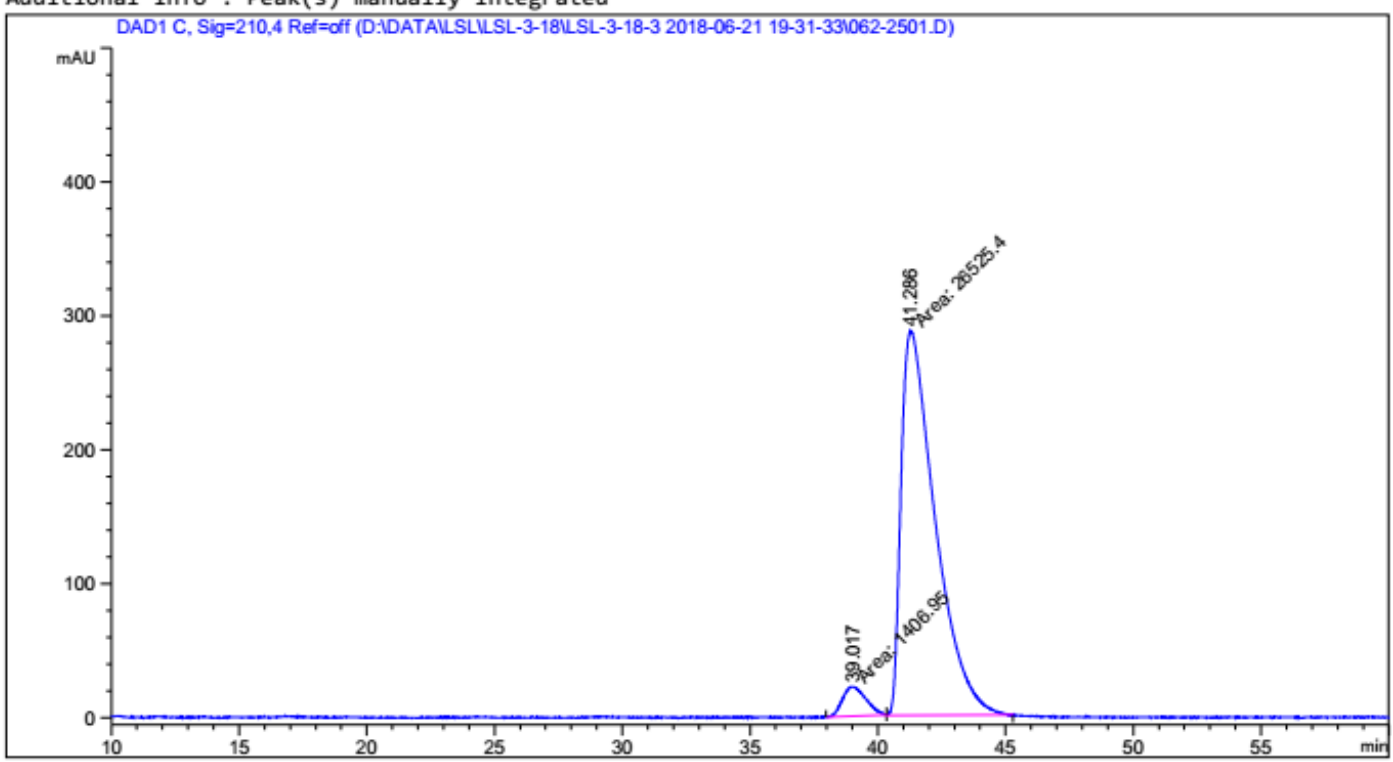

Area Percent Report

\begin{tabular}{|c|c|c|}
\hline Sorted By & : & Signal \\
\hline Multiplier & : & 1.0000 \\
\hline Dilution & : & 1.0000 \\
\hline
\end{tabular}

Use Multiplier \& Dilution Factor with ISTDs

Signal 1: DAD1 C, Sig $=210,4$ Ref $=0 f f$

\begin{tabular}{|c|c|c|c|c|c|}
\hline $\begin{array}{c}\text { Peak } \\
\#\end{array}$ & $\begin{array}{l}\text { RetTime Type } \\
\text { [min] }\end{array}$ & $\begin{array}{l}\text { Width } \\
\text { [min] }\end{array}$ & $\begin{array}{c}\text { Area } \\
{\left[\mathrm{mAU} \mathbf{A}^{*} \mathrm{~s}\right]}\end{array}$ & $\begin{array}{l}\text { Height } \\
\text { [mAU] }\end{array}$ & $\begin{array}{c}\text { Area } \\
\%\end{array}$ \\
\hline$\cdots$ & $|\cdots|$ & 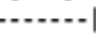 & 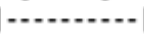 & | & | \\
\hline 1 & $39.017 \mathrm{MM}$ & 1.0604 & 1406.95313 & 22.11340 & 5.0370 \\
\hline 2 & $41.286 \mathrm{MM}$ & 1.5436 & $2.65254 \mathrm{e} 4$ & 286.40863 & 94.9630 \\
\hline Tota & : & & $2.79324 \mathrm{e} 4$ & 308.52203 & \\
\hline
\end{tabular}


<smiles>O=C1C[C@H](c2ccc3c(c2)OCCO3)CO1</smiles>

Data File D: \DATA LSL \LSL-3-18\LSL-3-18-6 2018-06-23 20-08-54\ө33-0301.D Sample Name: LSL-2-194-2

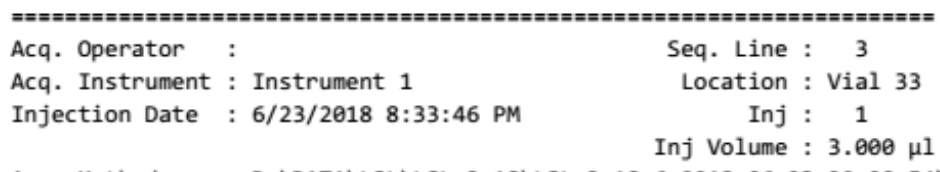

Acq. Method : D: \DATA $L S L \backslash L S L-3-18 \backslash L S L-3-18-6$ 2018-06-23 20-08-54 VWD-AD(1-2) -85-15-1ML3UL-220NM-3OMIN. M

Last changed : 6/23/2018 8:07:06 PM

Analysis Method : D: \METHOD\HZY \DAD-OD(1-2)-90-10-1ML-2UL-ALL-40MIN. M

Last changed : 9/22/2018 10:07:25 PM

(modified after loading)

Additional Info : Peak(s) manually integrated

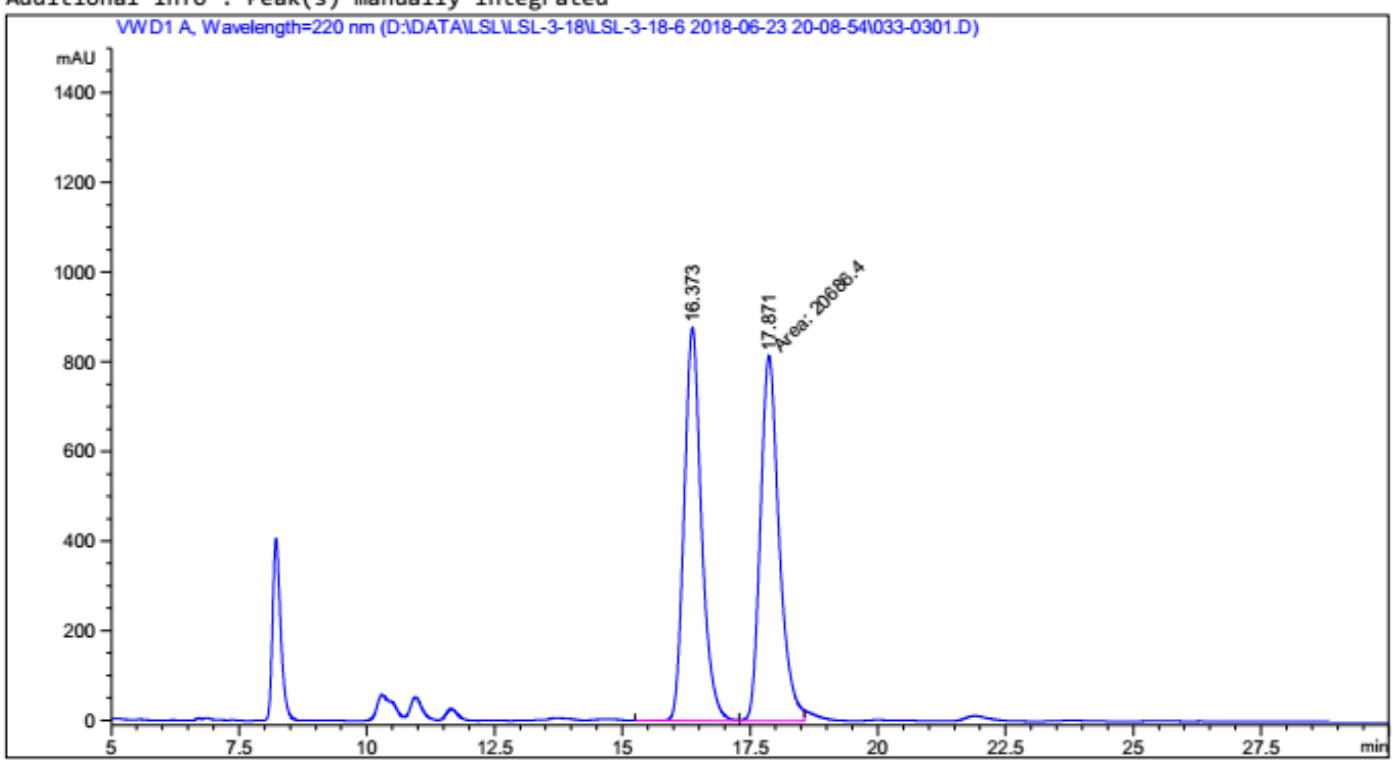

Area Percent Report

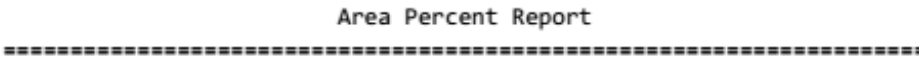

Sorted By : : Signal

Multiplier : 1.0000

Dilution : 1.0000

Use Multiplier \& Dilution Factor with ISTDs

Signal 1: VWD1 A, Wavelength $=220 \mathrm{~nm}$

\begin{tabular}{|c|c|c|c|c|c|}
\hline $\begin{array}{c}\text { Peak } \\
\#\end{array}$ & $\begin{array}{l}\text { RetTime Type } \\
\text { [min] }\end{array}$ & $\begin{array}{l}\text { Width } \\
\text { [min] }\end{array}$ & $\begin{array}{c}\text { Area } \\
{\left[\mathrm{mAU}{ }^{*} \mathrm{~s}\right]}\end{array}$ & $\begin{array}{l}\text { Height } \\
\text { [mAU] }\end{array}$ & $\begin{array}{c}\text { Area } \\
\%\end{array}$ \\
\hline & & & 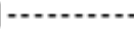 & 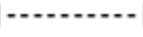 & | \\
\hline 1 & $16.373 \mathrm{BV}$ & 0.3569 & $2.06396 \mathrm{e} 4$ & 875.95856 & 49.9433 \\
\hline 2 & 17.871 MF & 0.4236 & $2.06864 \mathrm{e} 4$ & 813.92114 & 50.0567 \\
\hline Tota & : & & $4.13260 \mathrm{e} 4$ & 1689.87970 & \\
\hline
\end{tabular}


Data File D: \DATA \LSLILSL-3-18\LSL-3-18-6 2018-06-23 20-08-54\034-0401.D Sample Name: LSL-3-18-3

Acq. Operator :

Acq. Instrument : Instrument 1

Injection Date : 6/23/2018 9:04:35 PM

D: IDATAYLSL YLSL-3-18YLSL 3UL-220NM-3OMIN.M

Last changed : 6/23/2018 8:07:06 PM

Analysis Method : D: \METHOD HZY \DAD-OD(1-2)-90-10-1ML-2UL-ALL-40MIN.M

Last changed : 9/22/2018 10:09:36 PM

(modified after loading)

Additional Info : Peak(s) manually integrated

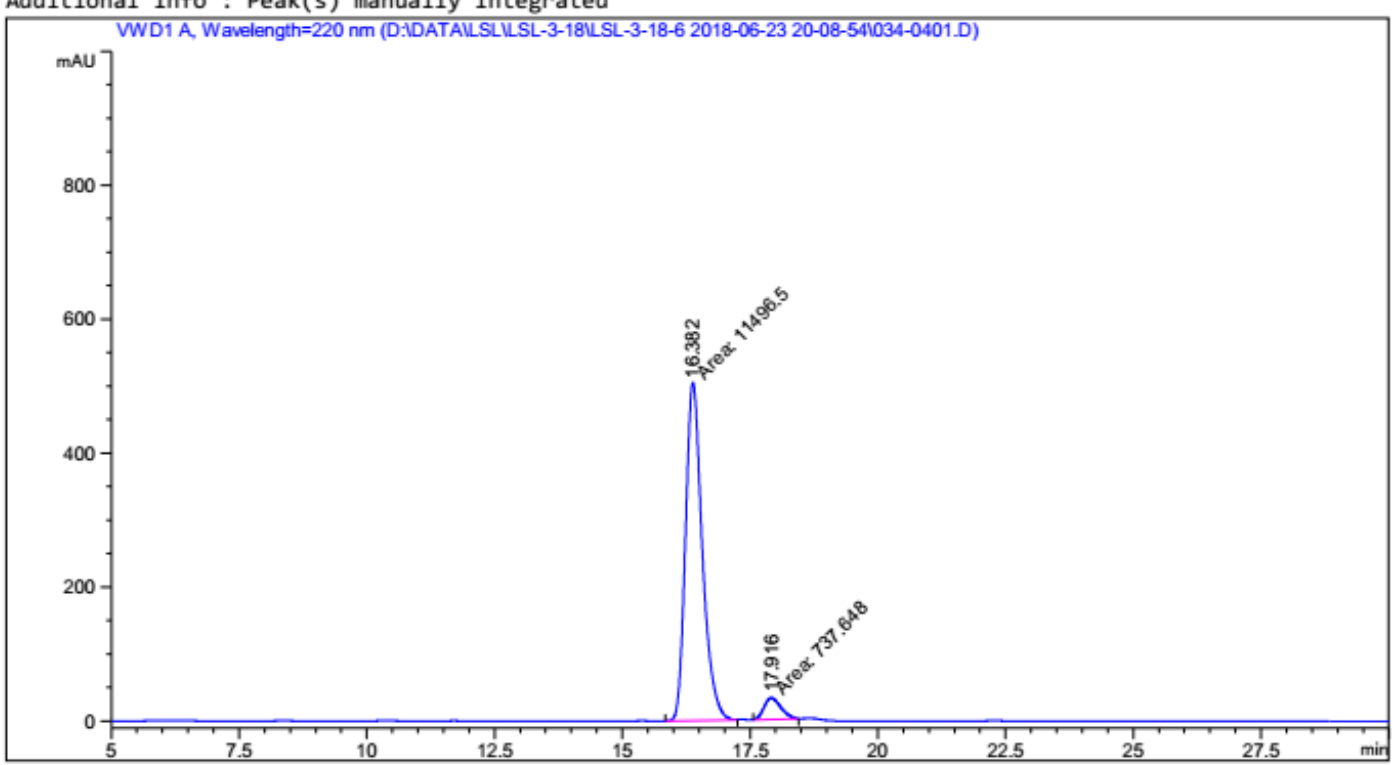

Area Percent Report

Sorted By : : Signal

Multiplier $\quad$ : 1.0000

1.0000
1.0000

Use Multiplier \& Dilution Factor with ISTDs

Signal 1: VWD1 A, Wavelength $=220 \mathrm{~nm}$

\begin{tabular}{|c|c|c|c|c|c|}
\hline $\begin{array}{c}\text { Peak } \\
\#\end{array}$ & $\begin{array}{l}\text { RetTime Type } \\
\text { [min] }\end{array}$ & $\begin{array}{l}\text { Width } \\
\text { [min] }\end{array}$ & $\begin{array}{c}\text { Area } \\
\text { [mAU*s] }\end{array}$ & $\begin{array}{l}\text { Height } \\
\text { [mAU] }\end{array}$ & $\begin{array}{c}\text { Area } \\
\%\end{array}$ \\
\hline & & & | & 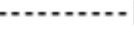 & 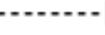 \\
\hline 1 & $16.382 \mathrm{MM}$ & 0.3799 & $1.14965 \mathrm{e} 4$ & 504.43137 & 93.9706 \\
\hline 2 & $17.916 \mathrm{MM}$ & 0.3820 & 737.64813 & 32.18591 & 6.0294 \\
\hline Tota. & : & & $1.22342 \mathrm{e} 4$ & 536.61727 & \\
\hline
\end{tabular}


<smiles>Cc1cc(C)cc(C2COC(=O)C2)c1</smiles>

Data File E: $\backslash$ DATA $\backslash X Z C \backslash A M I N 0-A C I D \backslash X Z C-20180409-1$ 2018-04-09 19-02-51\005-1001.D Sample Name: LSL-2-152-5

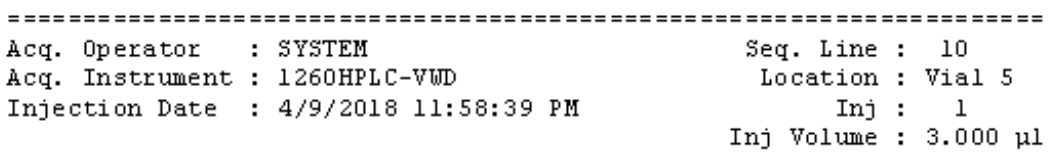

Acq. Me thod : E: \DATA \XZC \AMIN0-ACID XXZC-20180409-1 2018-04-09 19-02-51 VUD-AD (1-2)-99 $-1-1 \mathrm{ML}-3 \mathrm{UL}-220 \mathrm{NM}-60 \mathrm{MIN}$. M

Last changed : 4/9/2018 9:29:40 PM by SYSTEM

Analysis Method : E: \DATA \XZC \AMIN0-ACID XXZC-20180409-1 2018-04-09 19-02-51 VUD-AD (1-2)-99 -1-1ML-3UL-220MM-60MIN.M (Sequence Me thod)

Last changed : 5/8/2018 10:13:20 AM by SYsTEM

(modified after loading)

Additional Info : Peak (s) manually integrated

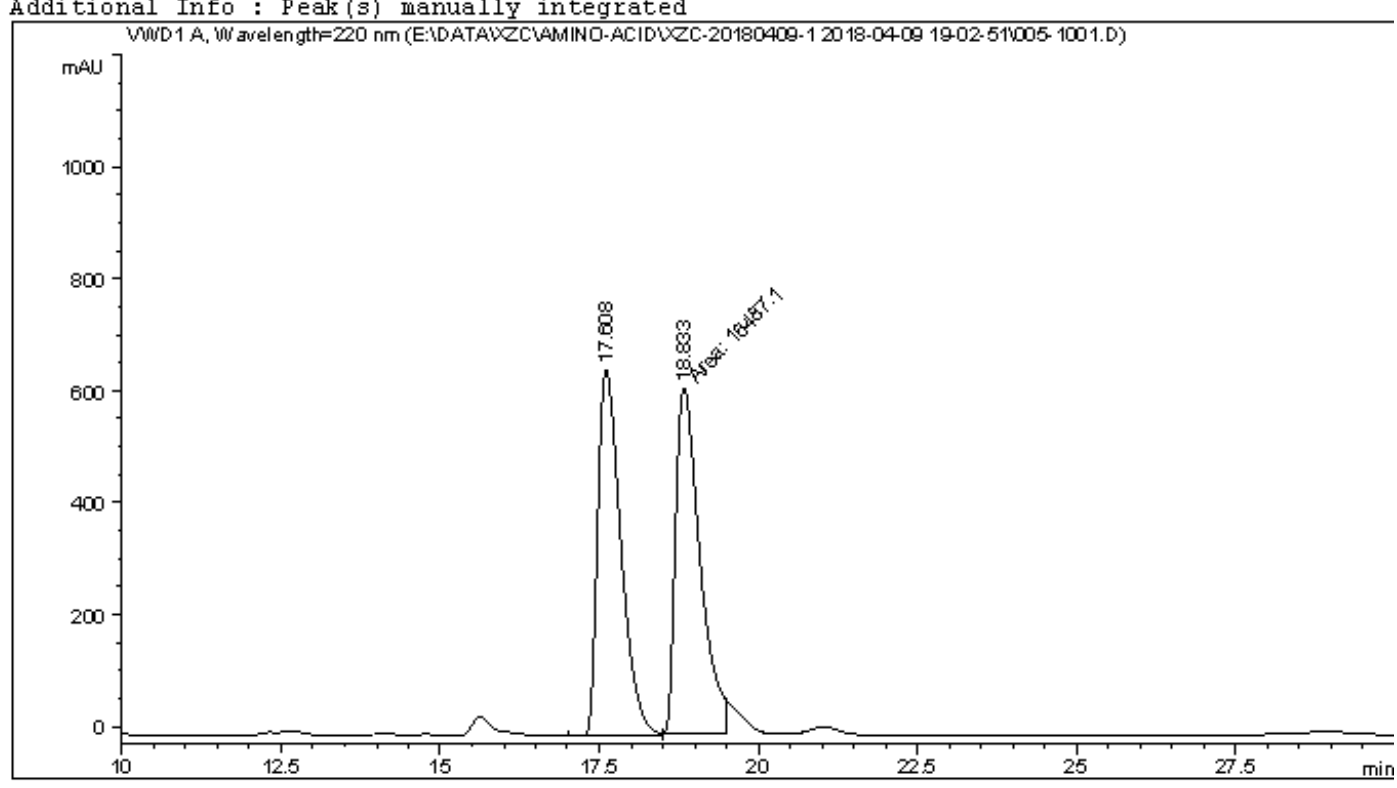

\section{Area Percent Report}

\begin{tabular}{|c|c|c|}
\hline Sorted By & : & signal \\
\hline Multiplier & : & 1. 0000 \\
\hline Dilution & : & 1. 0000 \\
\hline
\end{tabular}

Do not use Multiplier \& Dilution Factor with ISTD s

Signal 1: VWD $1 \mathrm{~A}$, Wave length $=220 \mathrm{~nm}$

\begin{tabular}{|c|c|c|c|c|c|c|}
\hline $\begin{array}{c}\text { Peak } \\
\#\end{array}$ & $\begin{array}{c}\text { Re tTime } \\
\text { [ } \min ]\end{array}$ & Type & $\begin{array}{l}\text { Width } \\
\text { [min] }\end{array}$ & $\begin{array}{c}\text { Area } \\
{\left[\text { [HÂU } U^{*} s\right]}\end{array}$ & $\begin{array}{l}\text { Height } \\
\text { [ mAU] }\end{array}$ & $\begin{array}{c}\text { Area } \\
\stackrel{4}{*}\end{array}$ \\
\hline 1 & 17.608 & BV & 0.3933 & 1. $65630 \mathrm{e} 4$ & 654.35492 & 50.1147 \\
\hline 2 & 18.833 & MF & 0.4437 & 1. $6487 \mathrm{le} 4$ & 619.27911 & 49.8853 \\
\hline & : & & & 3. $30501 \mathrm{e} 4$ & 1273.63403 & \\
\hline
\end{tabular}

\#* End of Report \#* 
Data File E: \DATA $\backslash$ LS \LSL-2-156\LSL-2-156 2018-04-14 21-53-52\015-0601.D Sample Name: LSL-2-156-5

\begin{tabular}{|c|c|c|c|}
\hline Acq. Operator & : SYSTEM & Seq. Line : & 6 \\
\hline Injection Date & : 4/15/2018 1:18:25 AM & $\begin{array}{r}\text { Inj } \\
\text { Inj Volume }\end{array}$ & $\begin{array}{c}1 \\
3.000\end{array}$ \\
\hline
\end{tabular}

Acq. Me thod : E: \DATA \LSL \LSL-2-156\LSL-2-156 2018-04-14 21-53-52 VWD-AD (1-2)-99-1-1ML $-3 \mathrm{UL}-22$ OMM-60MIN.M

Last changed : 4/14/2018 9:53:53 PM by SYSTEM

Analysis Method : E: \DATA \LSL \LL-2-156\LSL-2-156 2018-04-14 21-53-52 VWD-AD (1-2)-99-1-1ML -3UL-220MM-60MIN.M (Sequence Me thod)

Last changed : 5/8/2018 10:18:53 AM by SYSTEM

(modified after loading)

Additional Info : Peak (s) manually integrated
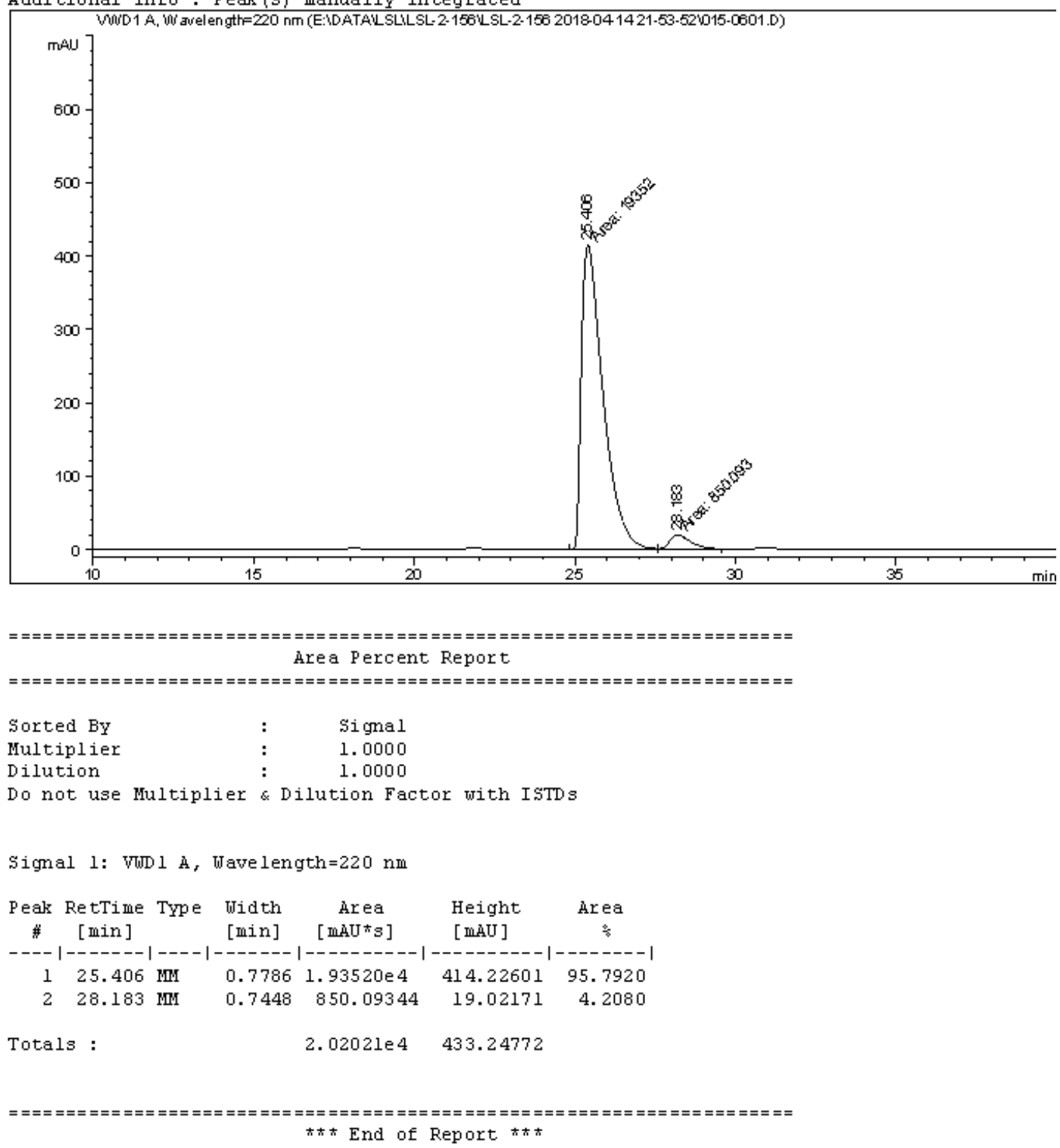
<smiles>O=C1C[C@H](c2ccc3ccccc3c2)CO1</smiles>

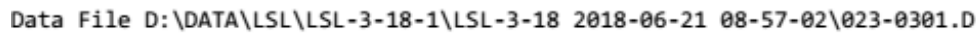
Sample Name: LSL-2-162-3

\begin{tabular}{|c|c|c|}
\hline Acq. Operator & : & Seq. Line : 3 \\
\hline Acq. Instrument & : Instrument 1 & Location : Vial 23 \\
\hline Injection Date & : 6/21/2018 9:23:17 AM & 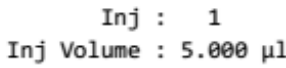 \\
\hline
\end{tabular}

Acq. Method : D: \DATA \LSLILSL-3-18-1\LSL-3-18 2018-06-21 08-57-02\VWD-AD(1-2)-99-1-1MLSUL-254NM-60MIN.M

Last changed : 5/29/2018 10:15:30 AM

Analysis Method : D: \METHOD\HZY \DAD-OD(1-2)-90-10-1ML-2UL-ALL-40MIN.M

Last changed : 9/22/2018 10:05:18 PM (modified after loading)

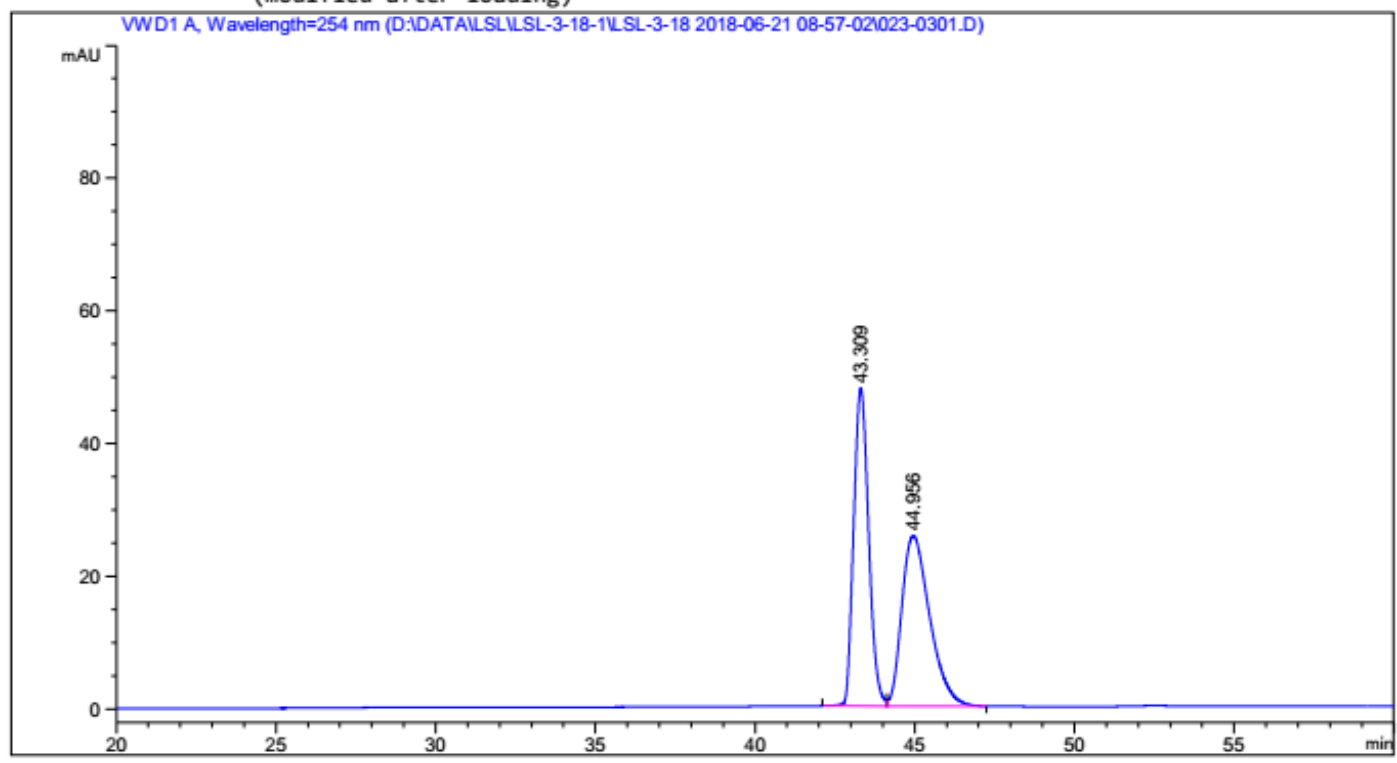

Area Percent Report

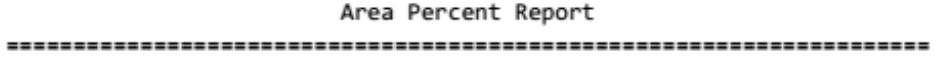

$\begin{array}{lll}\text { Sorted By } & : & \text { Signal } \\ \text { Multiplier } & : & 1.0000 \\ \text { Dilution } & : & 1.0000\end{array}$

Use Multiplier \& Dilution Factor with ISTDs

Signal 1: VWD1 A, Wavelength $=254 \mathrm{~nm}$

\begin{tabular}{|c|c|c|c|c|c|}
\hline $\begin{array}{c}\text { Peak } \\
\#\end{array}$ & $\begin{array}{l}\text { RetTime Type } \\
\text { [min] }\end{array}$ & $\begin{array}{l}\text { Width } \\
\text { [min] }\end{array}$ & $\begin{array}{c}\text { Area } \\
\text { [mAU*s] }\end{array}$ & $\begin{array}{l}\text { Height } \\
\text { [mAU] }\end{array}$ & $\begin{array}{c}\text { Area } \\
\%\end{array}$ \\
\hline$\cdots$ & $-\cdots+\mid \cdots$ & ............. & $\left|\begin{array}{lll}-1 & \ldots & n\end{array}\right|$ & | & | \\
\hline 1 & $43.309 \mathrm{BV}$ & 0.5132 & 1559.66248 & 47.85642 & 49.9517 \\
\hline 2 & $44.956 \mathrm{VB}$ & 0.9304 & 1562.67969 & 25.62745 & 50.0483 \\
\hline Tota & & & 3122.34216 & 73.48388 & \\
\hline
\end{tabular}




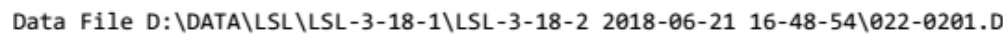
Sample Name: LSL-3-18-2

Acq. Operator :

Acq. Instrument : Instrument 1

Injection Date : 6/21/2018 5:00:42 PM
Seq. Line : 2

Location : Vial 22

Inj : 1

Inj Volume : $3.000 \mu$

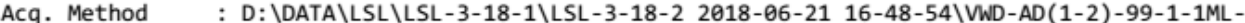
3UL-220NM-60MIN.M

Last changed : 5/28/2018 10:18:57 PM

Analysis Method : D: \METHOD\HZY \DAD-OD(1-2)-90-10-1ML-2UL-ALL-40MIN.M

Last changed : 9/22/2018 10:00:44 PM

(modified after loading)

Additional Info: Peak(s) manually integrated

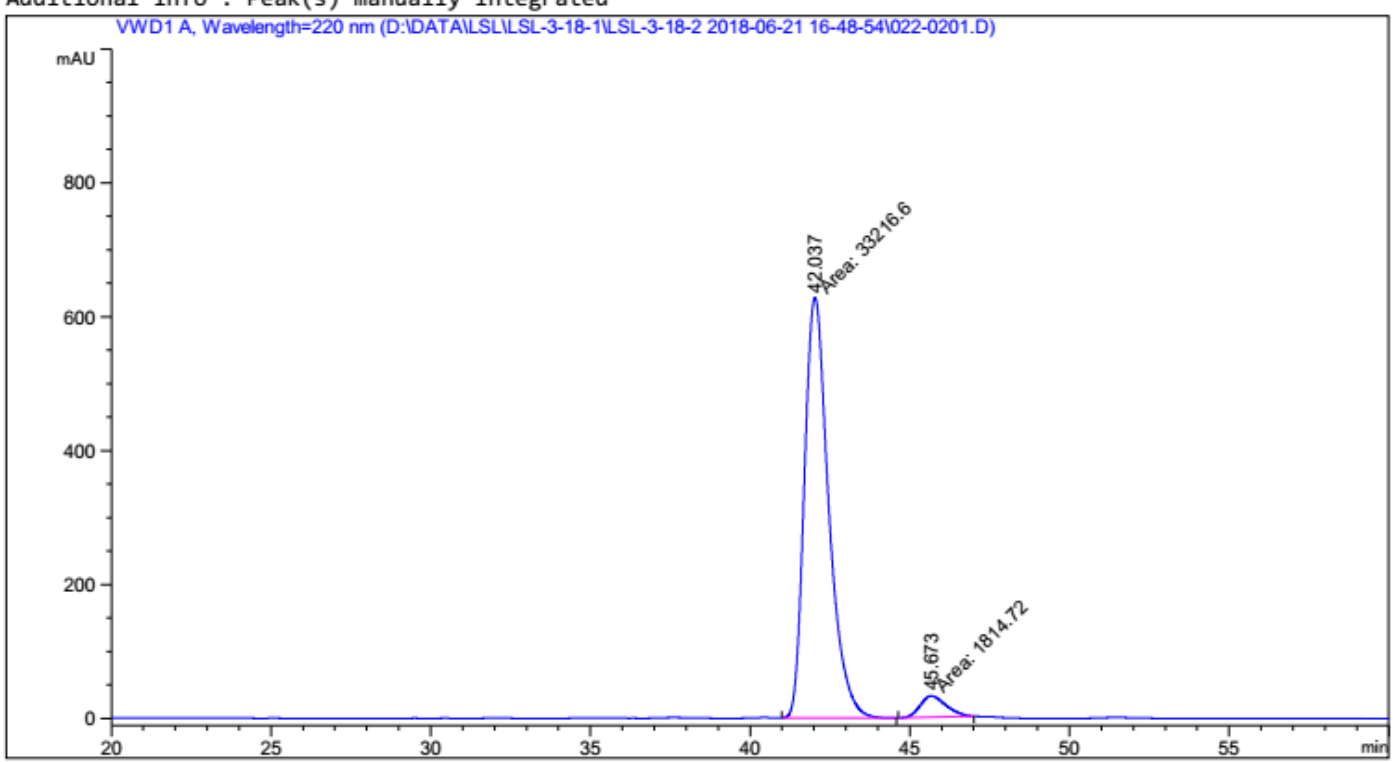

Area Percent Report

Sorted By : : Signal

Multiplier : $\quad 1.0000$

Dilution : 1.0000

Use Multiplier \& Dilution Factor with ISTDs

Signal 1: VWD1 A, Wavelength $=220 \mathrm{~nm}$

\begin{tabular}{|c|c|c|c|c|c|c|}
\hline $\begin{array}{c}\text { Peak } \\
\#\end{array}$ & $\begin{array}{c}\text { RetTime } \\
\text { [min] }\end{array}$ & Type & $\begin{array}{l}\text { Width } \\
\text { [min] }\end{array}$ & $\begin{array}{c}\text { Area } \\
{[\mathrm{mAU} * \mathrm{~s}]}\end{array}$ & $\begin{array}{l}\text { Height } \\
\text { [mAU] }\end{array}$ & $\begin{array}{c}\text { Area } \\
\%\end{array}$ \\
\hline$\cdots$ & ....... & & (n) & | & . & | \\
\hline 1 & 42.037 & MM & 0.8820 & $3.32166 \mathrm{e} 4$ & 627.67572 & 94.8197 \\
\hline 2 & 45.673 & MM & 0.9424 & 1814.72290 & 32.09282 & 5.1803 \\
\hline Tota] & : & & & $3.50313 \mathrm{e} 4$ & 659.76854 & \\
\hline
\end{tabular}




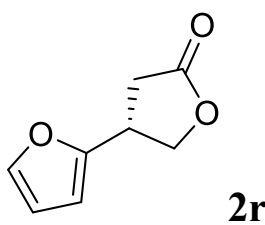

Data File E: \DATA \YJXYYJX-1-124-0504\YJX-1-124 2018-05-04 16-21-44\023-2201.D Sample Name: LSL-2-170-4

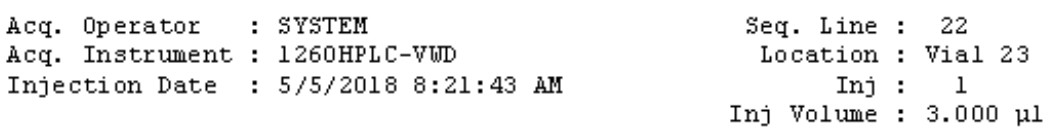

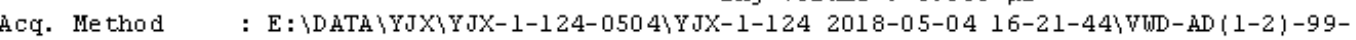
$1-1 M L-3 U L-220 M M-60 M I N . M$

Last changed : 5/4/2018 8:59:53 PM by SYSTEM

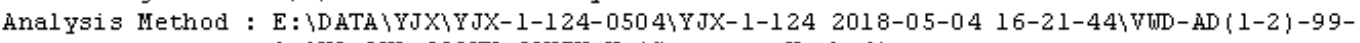
1-1ML-3UL-220NM-60MIN.M (Sequence Method)

Last changed : 5/5/2018 2:41:17 PM by 5Y STEM

(modified after loading)

Additional Info : Peak ( $s$ ) manually integrated

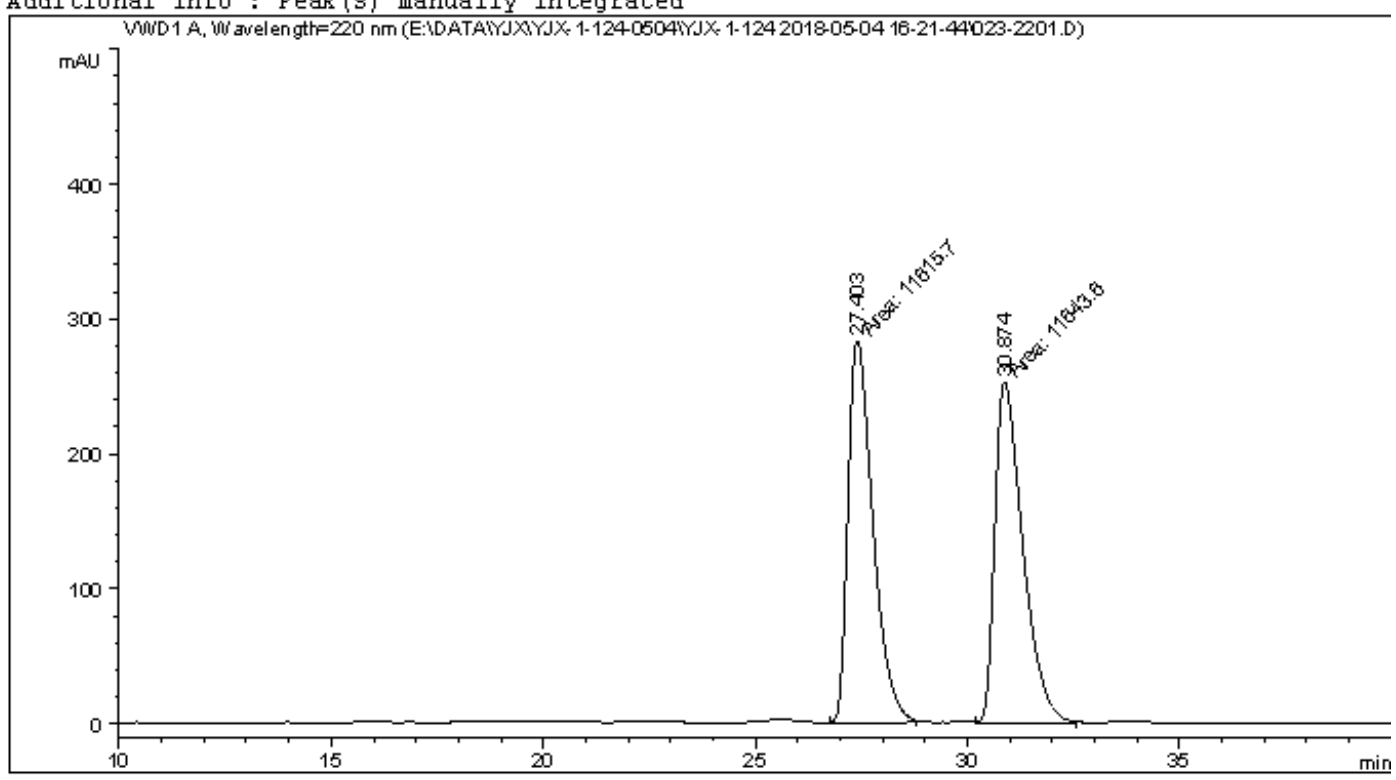

Area Percent Report

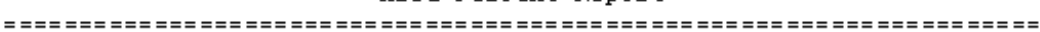

$\begin{array}{lll}\text { Sorted By } & : & \text { Signal } \\ \text { Multiplier } & : & 1.0000 \\ \text { Dilution } & : & 1.0000\end{array}$

Dilution : $\quad 1.0000$

Do not use Multiplier \& Dilution Factor with ISTD s

Signal 1: VWD $1 \mathrm{~A}$, Wave length $=220 \mathrm{~nm}$

\begin{tabular}{|c|c|c|c|c|c|c|}
\hline $\begin{array}{c}\text { Peak } \\
\#\end{array}$ & $\begin{array}{c}\text { RetTime } \\
\text { [min] }\end{array}$ & Type & $\begin{array}{l}\text { Width } \\
\text { [min] }\end{array}$ & $\begin{array}{c}\text { Area } \\
{\left[\mathrm{mAH} U^{*} s\right]}\end{array}$ & $\begin{array}{l}\text { Height } \\
\text { [ mAU] }\end{array}$ & $\begin{array}{c}\text { Area } \\
\text { s }\end{array}$ \\
\hline 1 & 27.403 & MM & 0.6825 & $1.16157 \mathrm{e} 4$ & 283.66660 & 49.9400 \\
\hline 2 & 30.874 & $M M$ & 0.7670 & 1. $16436 \mathrm{e} 4$ & 253.02267 & 50.0600 \\
\hline
\end{tabular}

$2.32593 e 4 \quad 536.68927$

*ネ* End of Report *ネ* 
Data File E: \DATA \YX\YJX-1-124-0504\YJX-1-124 2018-05-04 16-21-44\024-2301.D Sample Name: LSL-2-171-4

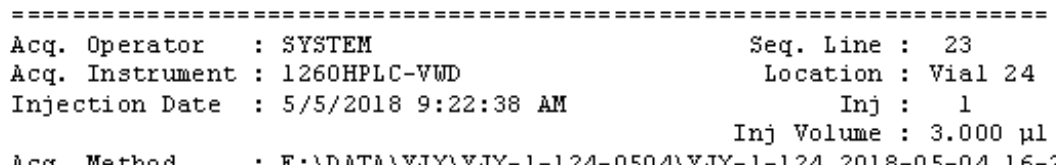

Acq. Me thod : E: \DATA \YJX\YJX-1-124-0504\YJX-1-124 2018-05-04 16-21-44\VWD-AD(1-2)-991-1ML-3UL-220MM-60M IN. M

Last changed : 5/4/2018 8:59:53 PM by SYSTEM

Analysis Method : E: \DATA \YJX\YJX-1-124-0504\YJX-1-124 2018-05-04 16-21-44\VWD-AD(1-2)-991-1ML-3UL-220MM-60MIN. M (Sequence Method)

Last changed : 5/5/2018 2:47:56 PM by SYSTEM

(modified after loading)

Additional Info : Peak (s) manually integrated
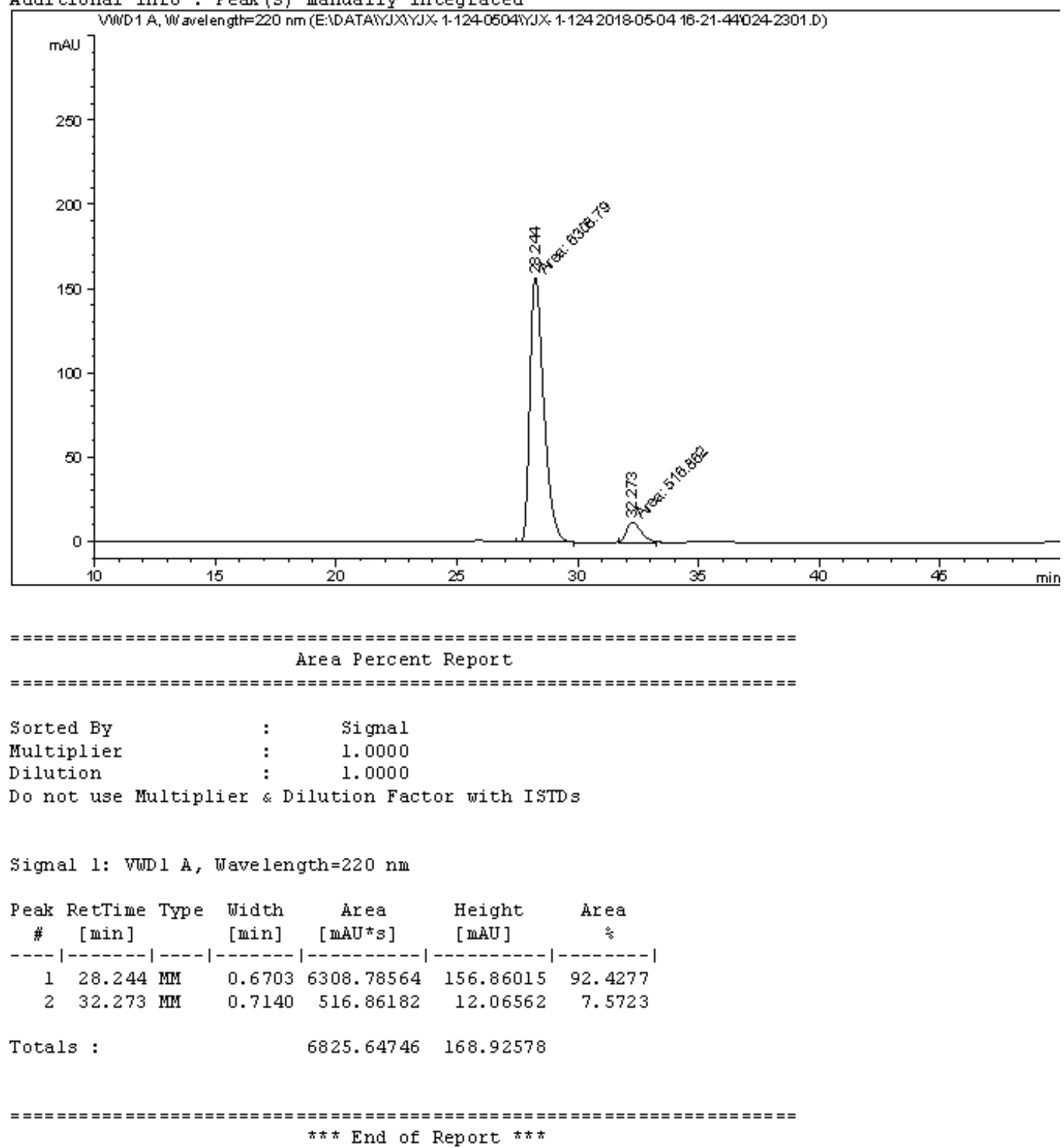
<smiles>O=C1C[C@H](c2ccc3occc3c2)CO1</smiles>

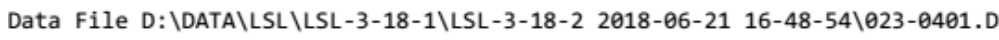
Sample Name: LSL-2-194-3

\begin{tabular}{|c|c|c|}
\hline $\begin{array}{l}\text { Acq. Operator } \\
\text { Acq. Instrument }\end{array}$ & : Instrument 1 & $\begin{array}{r}\text { Seq. Line : } 4 \\
\text { Location : Vial } 23\end{array}$ \\
\hline Injection Date & : 6/21/2018 6:14:45 PM & $\begin{array}{rc}\text { Inj }: & 1 \\
\text { Inj Volume } & : 3.000 \mu\end{array}$ \\
\hline
\end{tabular}

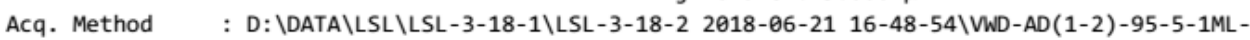
3UL-220NM-40MIN.M

Last changed : 6/7/2018 11:25:33 AM

Analysis Method : D: \METHOD \HZY \DAD-OD(1-2)-90-10-1ML-2UL-ALL-40MIN.M

Last changed : 9/22/2018 9:52:33 PM

(modified after loading)

Additional Info : Peak(s) manually integrated

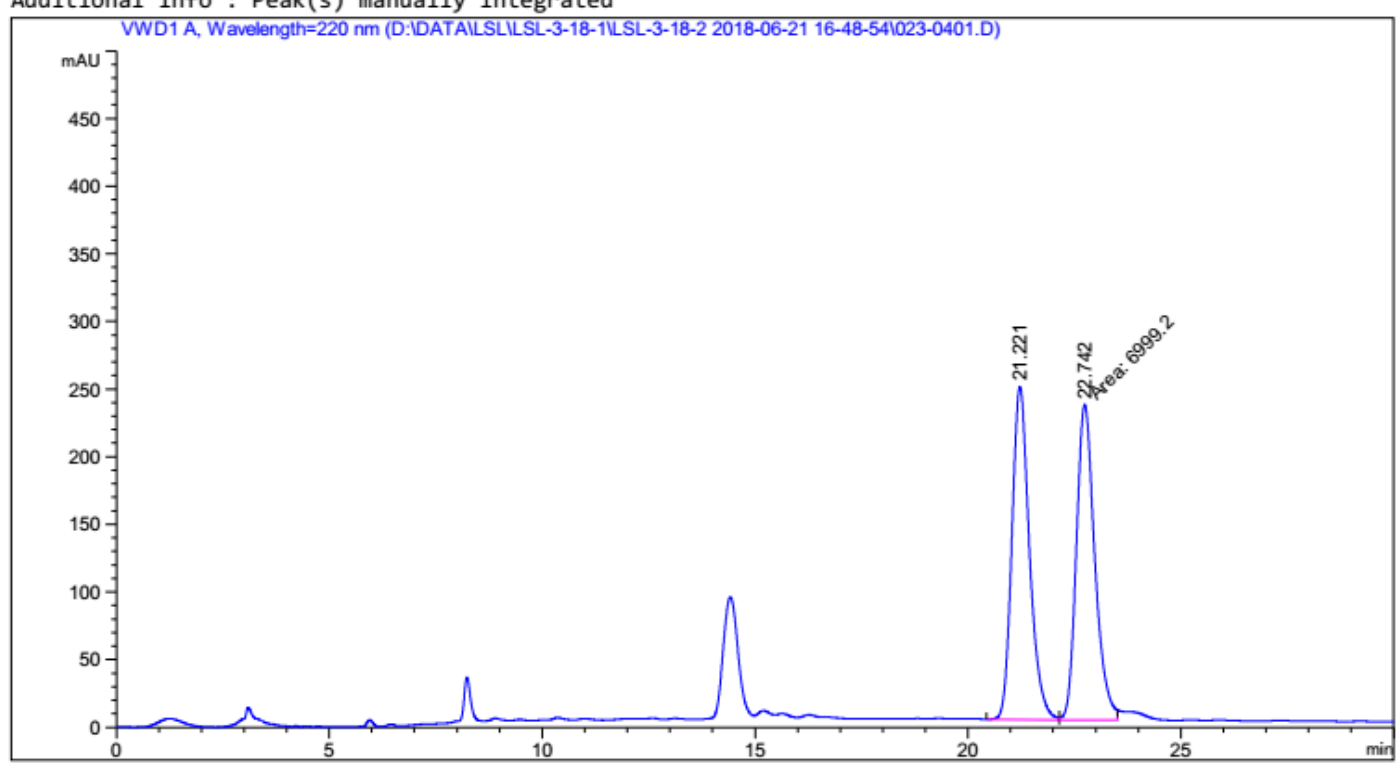

Area Percent Report

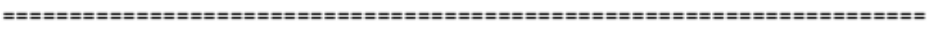

Sorted By : : Signal

Multiplier : $\quad 1.0000$

Dilution : 1.0000

Use Multiplier \& Dilution Factor with ISTDs

Signal 1: VWD1 A, Wavelength $=220 \mathrm{~nm}$

\begin{tabular}{|c|c|c|c|c|c|}
\hline $\begin{array}{c}\text { Peak } \\
\#\end{array}$ & $\begin{array}{l}\text { RetTime Type } \\
\text { [min] }\end{array}$ & $\begin{array}{l}\text { Width } \\
\text { [min] }\end{array}$ & $\begin{array}{c}\text { Area } \\
{\left[\mathrm{mAU}^{*} \mathrm{~s}\right]}\end{array}$ & $\begin{array}{l}\text { Height } \\
\text { [mAU] }\end{array}$ & $\begin{array}{c}\text { Area } \\
\%\end{array}$ \\
\hline$\ldots$ & $-\cdots$ & . & | & | & | \\
\hline 1 & $21.221 \mathrm{BV}$ & 0.4338 & 7037.46973 & 245.96678 & 50.1363 \\
\hline 2 & $22.742 \mathrm{MF}$ & 0.5003 & 6999.20020 & 233.17760 & 49.8637 \\
\hline Tot & : & & $1.40367 \mathrm{e} 4$ & 479.14438 & \\
\hline
\end{tabular}




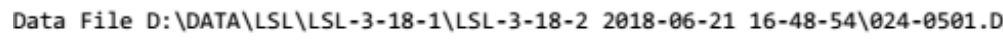
Sample Name: LSL-3-18-4

Acq. Operator :

Acq. Instrument : Instrument 1

Injection Date : 6/21/2018 6:55:34 PM$$
\text { Inj Volume : } 3.000 \mu 1
$$

Seq. Line : 5

Location : Vial 24

Inj : 1

Inj Volume : $3.000 \mu 1$

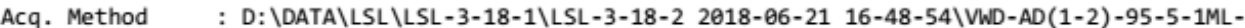
3UL-220NM-40MIN.M

Last changed : 6/7/2018 11:25:33 AM

Analysis Method : D: \METHOD\HZY \DAD-OD(1-2)-90-10-1ML-2UL-ALL-40MIN.M

Last changed : 9/22/2018 9:56:56 PM

(modified after loading)

Additional Info : Peak(s) manually integrated

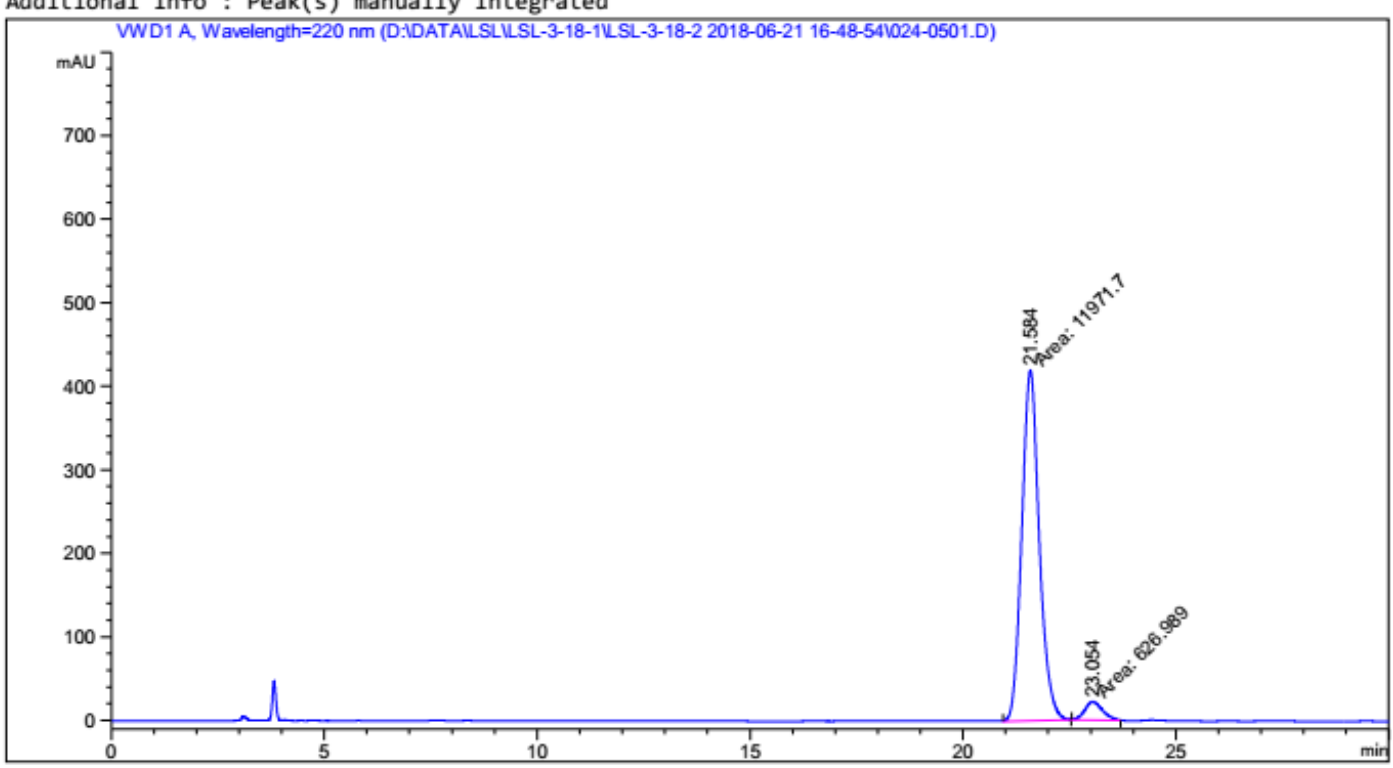

Area Percent Report

Sorted By : : Signal

Multiplier : $\quad 1.0000$

Dilution : 1.0000

Use Multiplier \& Dilution Factor with ISTDs

Signal 1: VWD1 A, Wavelength $=220 \mathrm{~nm}$

\begin{tabular}{|c|c|c|c|c|c|}
\hline $\begin{array}{c}\text { Peak } \\
\#\end{array}$ & $\begin{array}{l}\text { RetTime Type } \\
\text { [min] }\end{array}$ & $\begin{array}{l}\text { Width } \\
\text { [min] }\end{array}$ & $\begin{array}{c}\text { Area } \\
{[\mathrm{mAU} * \mathrm{~s}]}\end{array}$ & $\begin{array}{l}\text { Height } \\
\text { [mAU] }\end{array}$ & $\begin{array}{c}\text { Area } \\
\%\end{array}$ \\
\hline & & & & & \\
\hline 1 & $21.584 \mathrm{MM}$ & 0.4756 & $1.19717 \mathrm{e} 4$ & 419.52008 & 95.0234 \\
\hline 2 & $23.054 \mathrm{MM}$ & 0.4809 & 626.98938 & 21.72960 & 4.9766 \\
\hline Tota & : & & $1.25987 \mathrm{e} 4$ & 441.24968 & \\
\hline
\end{tabular}


<smiles>[X]C1COC(=O)C1</smiles>

Data File E: \DATA \YJX YJX-1-124-0507\YJX-1-124-0507 2018-05-07 15-35-37\021-0401.D Sample Name: LSL-2-170-3

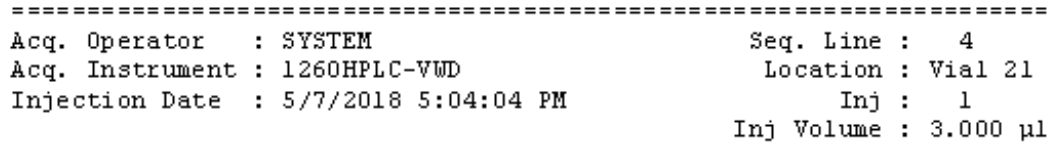

Acq. Me thod : E: \DATA \YJX\YJX-1-124-0507\YJX-1-124-0507 2018-05-07 15-35-37\VID-AD (1-2 -90-10-1ML-3UL-220MM-40M IN. M

Last changed : 5/7/2018 4:37:51 PM by SYSTEM

Analysis Method : E: \DATA \YJX\YJX-1-124-0507\YJX-1-124-0507 2018-05-07 15-35-37\VJD-AD (1-2

Last changed : 5/7/2018 9:31:07 PM by 5Y STEM

(modified after loading)

Additional Info : Peak ( $s$ ) manually integrated

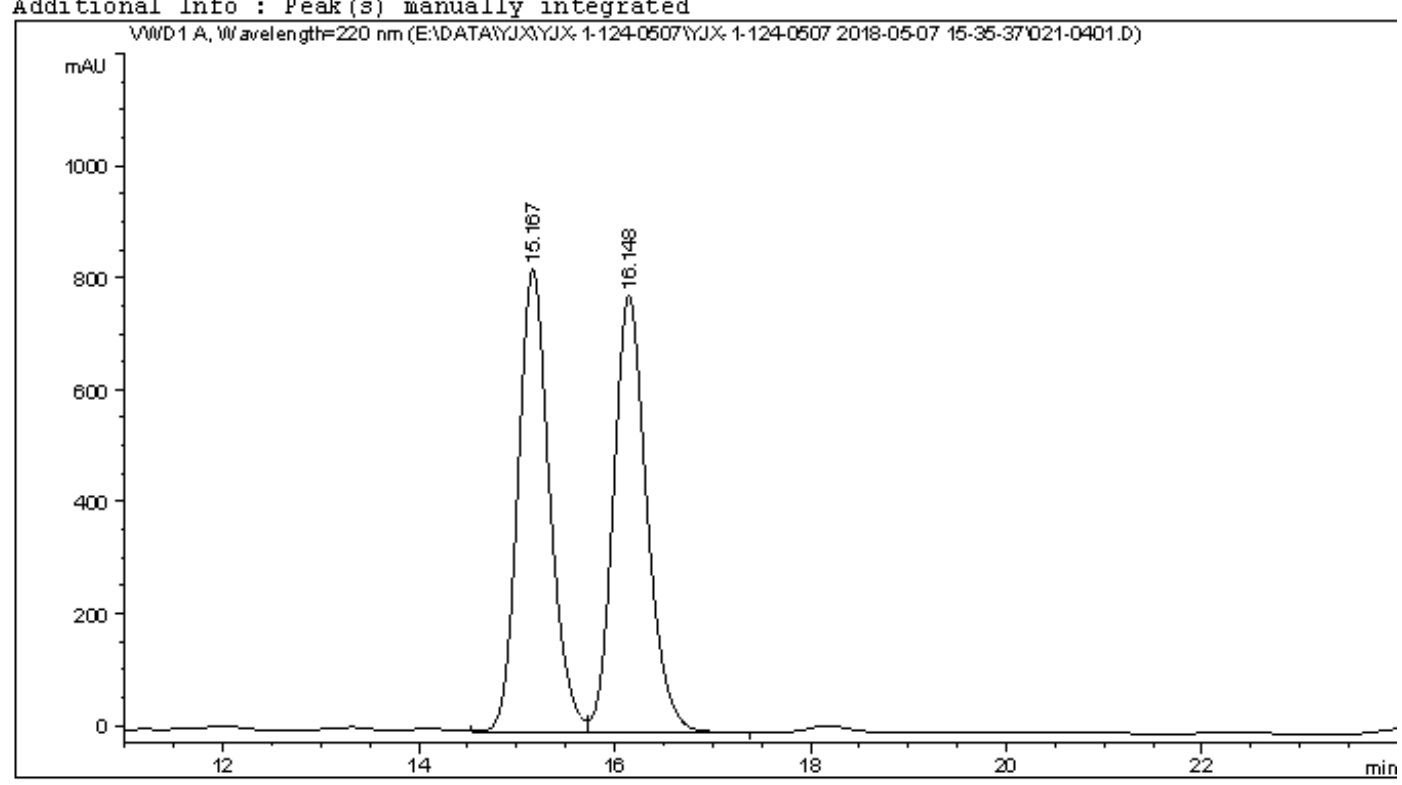

Area Percent Report

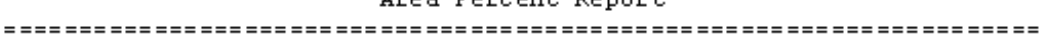

$\begin{array}{lll}\text { Sorted BY } & : & \text { Signal } \\ \text { Multiplier } & : & 1.0000 \\ \text { Dilution } & : & 1.0000\end{array}$

Dilution : 1.0000

Do not use Multiplier \& Dilution Factor with ISTD s

Signal 1: VWD 1 A, Wave length $=220 \mathrm{~nm}$

\begin{tabular}{|c|c|c|c|c|c|c|}
\hline $\begin{array}{c}\text { Peak } \\
\#\end{array}$ & $\begin{array}{c}\text { RetTime } \\
\text { [min] }\end{array}$ & Type & $\begin{array}{l}\text { Width } \\
\text { [min] }\end{array}$ & $\begin{array}{c}\text { Area } \\
{\left[\mathrm{mAU}^{*} \mathrm{~s}\right]}\end{array}$ & $\begin{array}{l}\text { Height } \\
\text { [ [MAU] }\end{array}$ & Area \\
\hline 1 & 15.167 & BV & 0.3428 & 1. $85445 \mathrm{e} 4$ & 826.28046 & 50.0977 \\
\hline 2 & 16.148 & VB & 0.3616 & 1. $84722 \mathrm{e} 4$ & 779.22522 & 49.9023 \\
\hline ota & 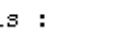 & & & 3. $70167 \mathrm{e} 4$ & 0568 & \\
\hline
\end{tabular}

ネネ* End of Report *ネ* 
Data File E: DATA YJX\YJX-1-124-0507\YJX-1-124-0507 2018-05-07 15-35-37\022-0501.D Sample Name: LSL-2-171-3

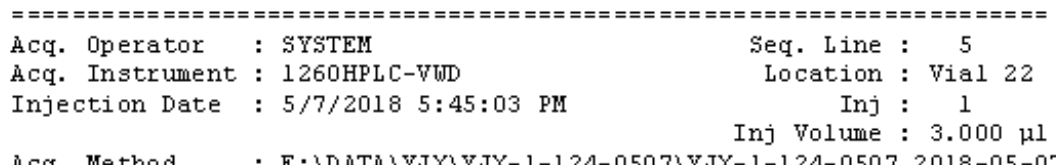

Acq. Me thod : E: \DATA \YJX)YJX-1-124-0507\YJX-1-124-0507 2018-05-07 15-35-37\VWD-AD (1-2 ) $-90-10-1 M L-3 U L-220$ MM- 40M IN. M

Last changed : 5/7/2018 4:37:51 PM by SYSTEM

Analysis Method : E: \DATA \YJX)YJX-1-124-0507\YJX-1-124-0507 2018-05-07 15-35-37)VWD-AD (1-2

Last changed : $5 / 7 / 20189: 31: 07$ PM by SYSTEM

(modified after loading)

Additional Info : Peak (s) manually integrated
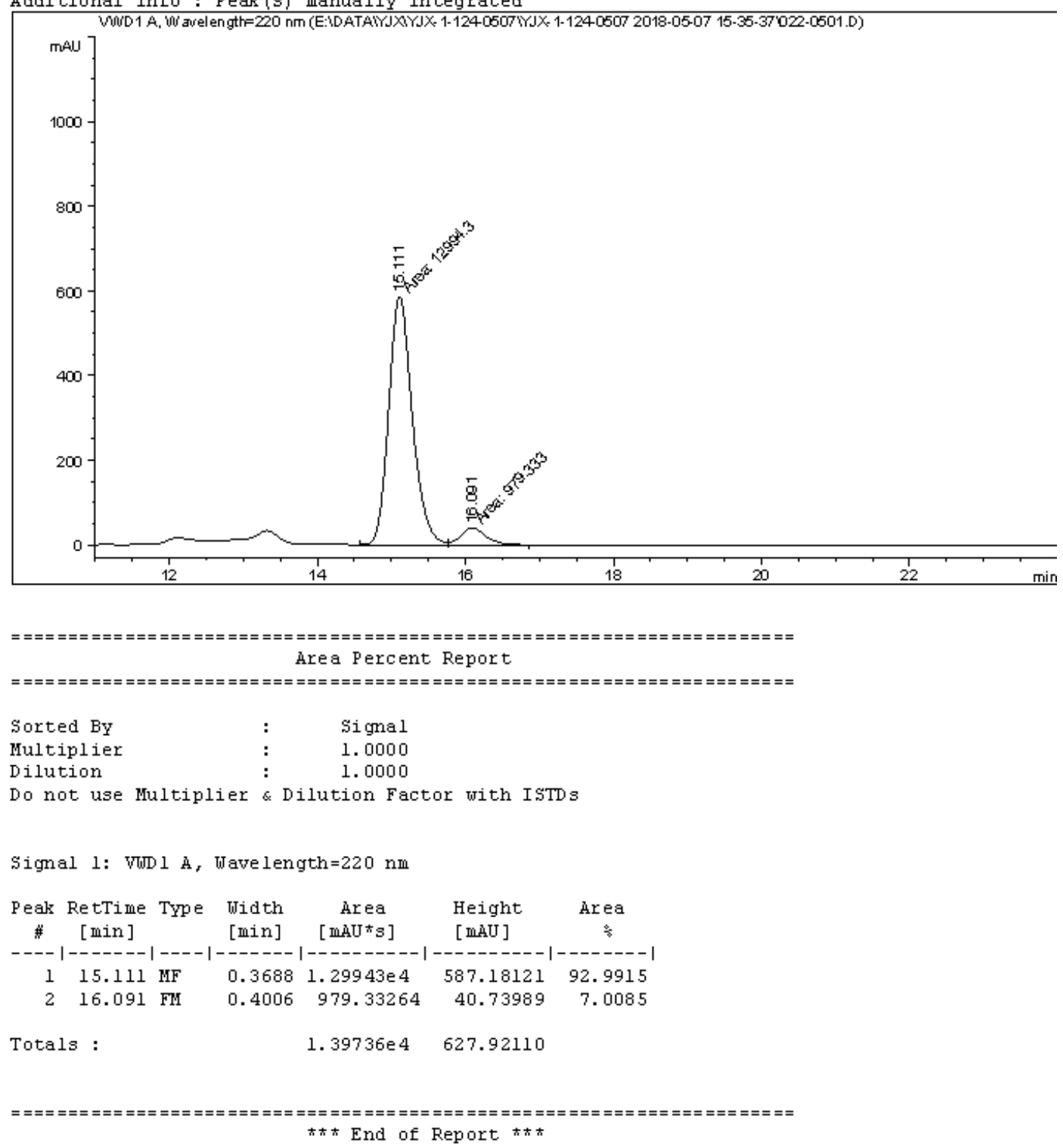
<smiles>CN1C[C@H](c2ccccc2)CC1=O</smiles>

$4 a$

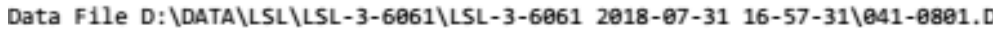
Sample Name: LSL-3-60-1

\begin{tabular}{|c|c|c|}
\hline Acq. Operator & & Seq. Line : 8 \\
\hline Acq. Instrument & : Instrument 1 & Location : Vial 41 \\
\hline Injection Date & : 7/31/2018 $8: 45: 25 \mathrm{PM}$ & 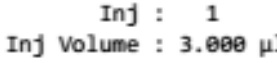 \\
\hline
\end{tabular}

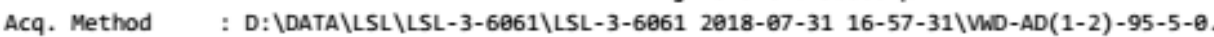
5ML-3UL-22ENM-60MIN .M

Last changed : 7/23/2018 8:16:17 AM

Analysis Method : D: METHOO\LWD \WD-AD(1-2)-80-20-1ML-3UL-210NM-60MIN.M

Last changed : 9/25/2018 10:12:21 AM

(modified after loading)

Additional Info: Peak(s) manually integrated

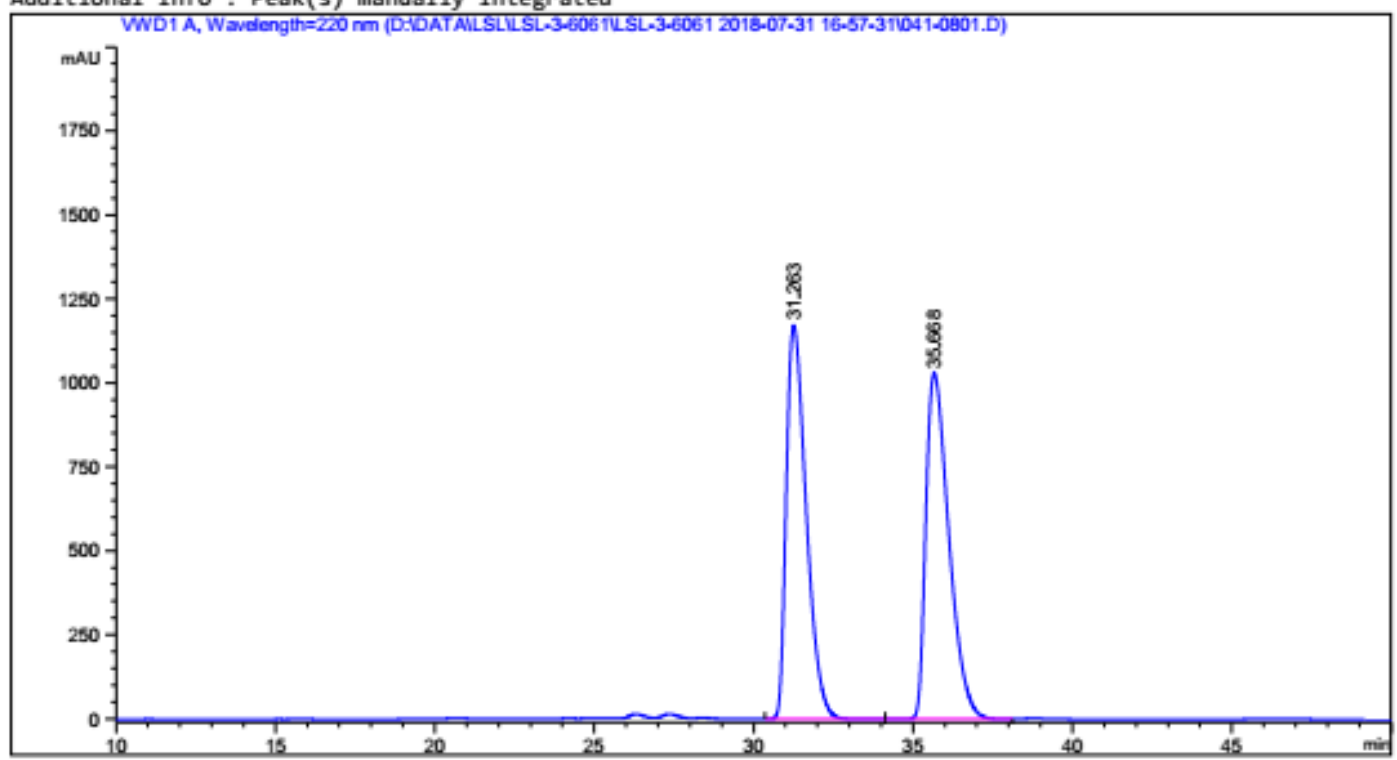

Area Percent Report

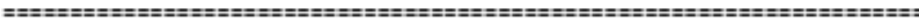

$\begin{array}{lll}\text { Sorted By } & : & \text { Signal } \\ \text { Multiplier } & : & 1.0000 \\ \text { Dilution } & : & 1.0000\end{array}$

Use Multiplier \& Dilution Factor with ISTDs

Signal 1: VWD1 A, Wavelength $=220 \mathrm{~nm}$

\begin{tabular}{|c|c|c|c|c|c|}
\hline $\begin{array}{c}\text { Peak } \\
\#\end{array}$ & $\begin{array}{l}\text { RetTime Type } \\
\text { [min] }\end{array}$ & $\begin{array}{l}\text { Width } \\
\text { [min] }\end{array}$ & $\begin{array}{c}\text { Area } \\
{\left[\mathrm{mAU}^{*} \mathrm{~s}\right]}\end{array}$ & $\begin{array}{l}\text { Height } \\
\text { [maU] }\end{array}$ & $\begin{array}{c}\text { Area } \\
\mathbf{\%}\end{array}$ \\
\hline & & & |-1-nen & |-cosen & \\
\hline 1 & $31.263 \mathrm{BB}$ & 0.6736 & $5.67399 \mathrm{e} 4$ & 1170.84180 & 49.6014 \\
\hline 2 & $35.668 \mathrm{BB}$ & 0.7776 & $5.15554 \mathrm{e} 4$ & 1028.86389 & 50.3986 \\
\hline Tota. & : & & $1.02295 \mathrm{e} 5$ & 2199.70569 & \\
\hline
\end{tabular}


Data File D: \DATA \LSL\LSL-3-6061\LSL-3-6061 2018-07-31 16-57-31\042-0901.D Sample Name: LSL-3-46-1

Acq. Operator :

Acq. Instrument : Instrument 1

Injection Date : 7/31/2018 9:46:16 PM

5ML-3UL-220NM-60MIN. M

Last changed : 7/23/2018 8:16:17 AM

Analysis Method: D: \METHOD\LWD \WD-AD(1-2)-80-20-1ML-3UL-210NM-60MIN. M

Last changed : 9/25/2018 10:18:31 AM

(modified after loading)

Additional Info : Peak(s) manually integrated

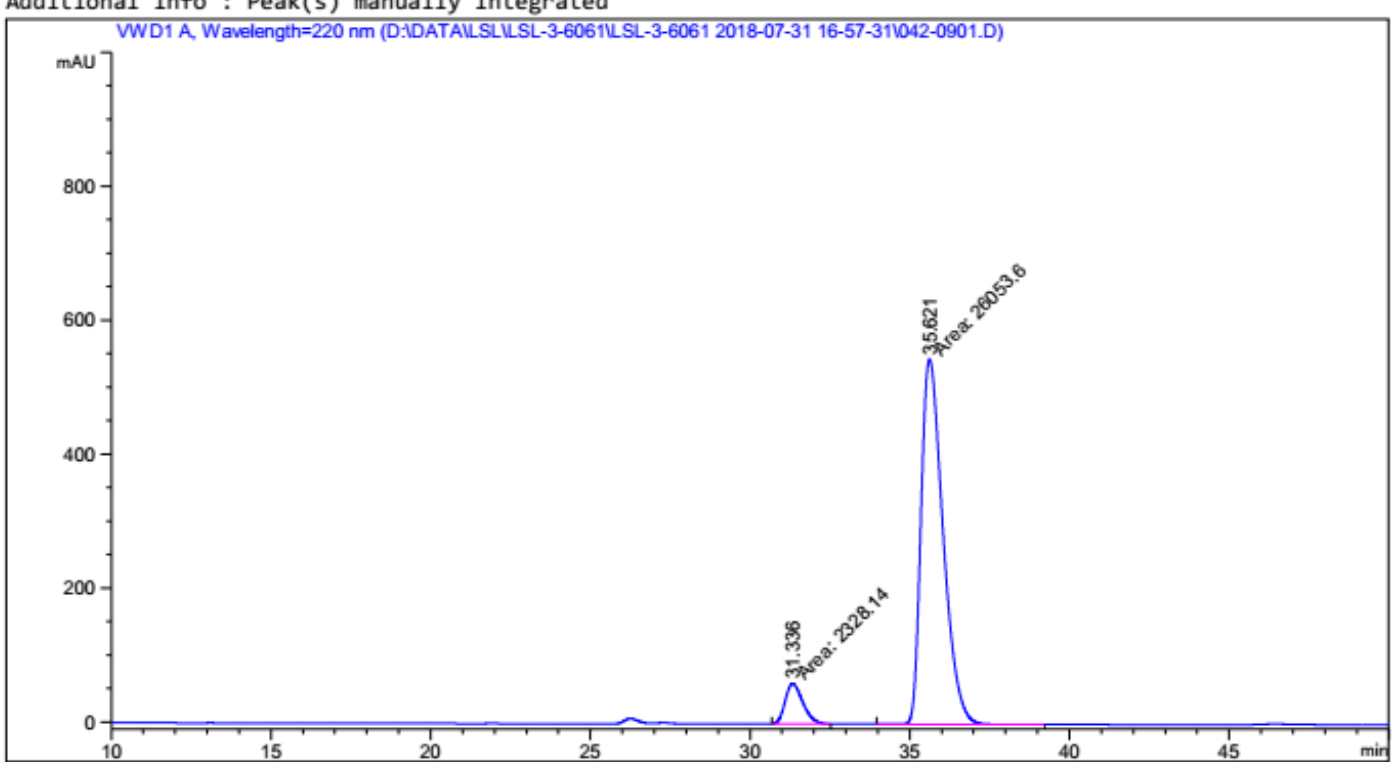

Area Percent Report

Sorted By : : Signal

Multiplier $\quad$ : 1.0000

1.0000

Use Multiplier \& Dilution Factor with ISTDs

Signal 1: VWD1 A, Wavelength $=220 \mathrm{~nm}$

Peak RetTime Type Width Area Height Area

\# [min] [min] [mAU*s] [mAU]

\%

- - - |

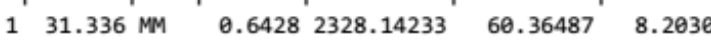

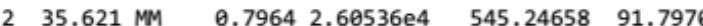

Totals : $\quad 2.83817 \mathrm{e} 4 \quad 605.61145$ 
<smiles>Cc1ccc([C@H]2CC(=O)N(C)C2)cc1</smiles>

4b

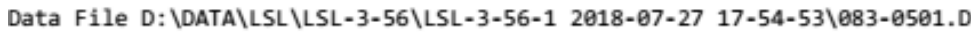
Sample Name: LSL-3-56-2

\begin{tabular}{|c|c|c|}
\hline $\begin{array}{l}\text { Acq. Operator } \\
\text { Acq. Instrument }\end{array}$ & : Instrument 1 & $\begin{aligned} & \text { Seq. Line : } 5 \\
& \text { Location : Vial } 83\end{aligned}$ \\
\hline Injection Date & : 7/27/2018 7:42:34 PM & $\begin{array}{rc}\text { Inj : } & 1 \\
\text { Inj Volume } & 3.000 \mu 1\end{array}$ \\
\hline
\end{tabular}

Acq. Method : D: \DATA \LSLILSL-3-56\LSL-3-56-1 2018-07-27 17-54-53\VWD-AD(1-2)-95-5-1ML3UL-220NM-46MIN.M

Last changed : 6/7/2018 11:25:33 AM

Analysis Method: D: METHOD\LWD\VWD-AD(1-2)-80-20-1ML-3UL-210NM-60MIN. M

Last changed : 9/25/2018 10:32:14 AM (modified after loading)

Additional Info: Peak(s) manually integrated

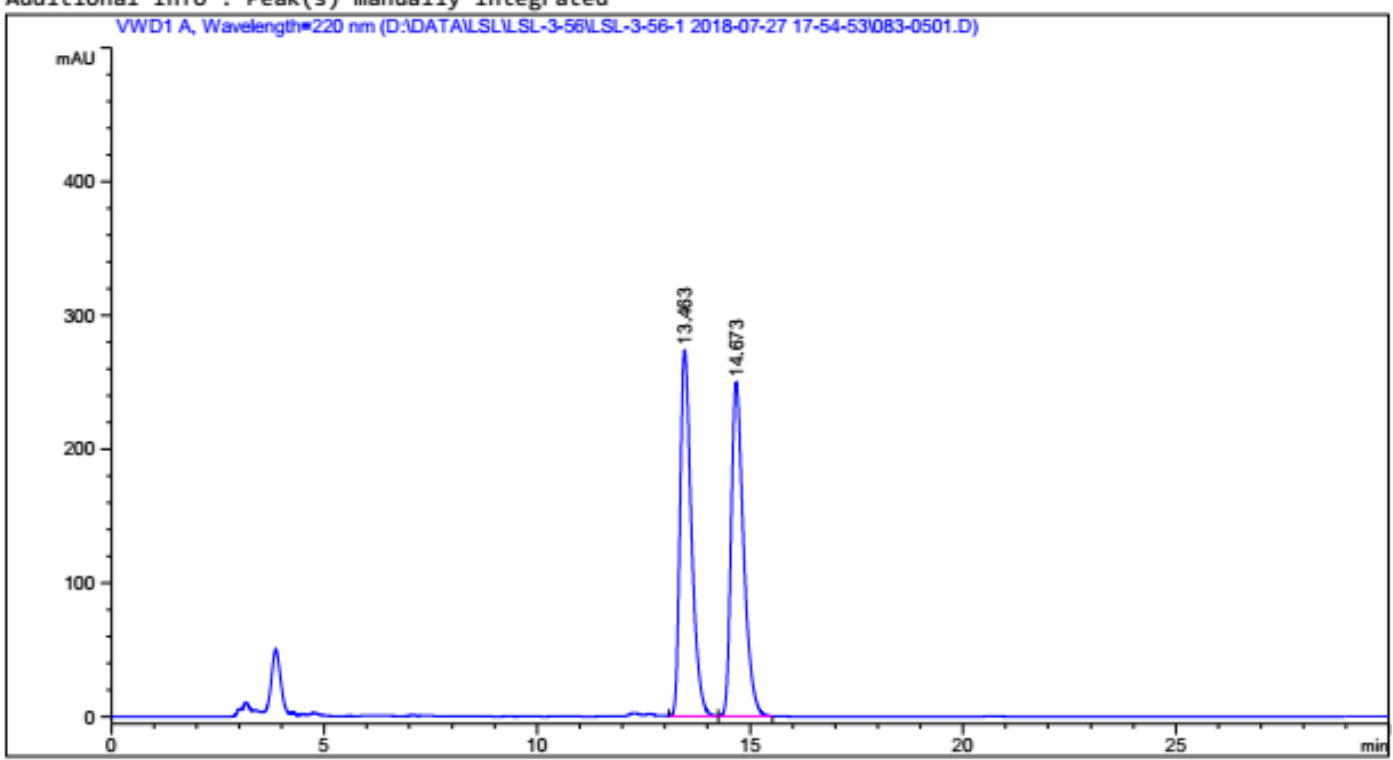

Area Percent Report

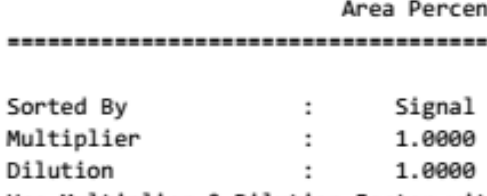

Use Multiplier \& Dilution Factor with ISTDs

Signal 1: WD1 A, Wavelength $=220 \mathrm{~nm}$

\begin{tabular}{|c|c|c|c|c|c|c|}
\hline $\begin{array}{c}\text { Peak } \\
\#\end{array}$ & $\begin{array}{c}\text { RetTime } \\
\text { [min] }\end{array}$ & Type & $\begin{array}{l}\text { Width } \\
\text { [min] }\end{array}$ & $\begin{array}{c}\text { Area } \\
\text { [mAU*s] }\end{array}$ & $\begin{array}{l}\text { Height } \\
\text { [mAU] }\end{array}$ & $\begin{array}{c}\text { Area } \\
\%\end{array}$ \\
\hline$\ldots$ & .......... & & (........ & |................ & . & .............. \\
\hline 1 & 13.463 & BB & 0.2808 & 5056.70020 & 273.40695 & 50.0090 \\
\hline 2 & 14.673 & BB & 0.3077 & 5054.88916 & 249.14897 & 49.9910 \\
\hline Tota & : & & & $1.01116 \mathrm{e} 4$ & 522.55592 & \\
\hline
\end{tabular}


Data File D: \DATA \LSLILSL-3-56\LSL-3-56-1 2018-07-27 17-54-53\084-0601.D Sample Name: LSL-3-46-2

Acq. Operator :

Acq. Instrument : Instrument 1

Injection Date : 7/27/2018 8:23:21 PM

D: \DATA ILSL \LSL-3-56 3UL-220NM-40MIN.M

Last changed : 6/7/2018 11:25:33 AM

Analysis Method: D: \METHOD\LWD \WWD-AD(1-2)-80-20-1ML-3UL-210NM-60MIN. M

Last changed

: 9/25/2018 10:35:13 AM

(modified after loading)

Additional Info : Peak(s) manually integrated

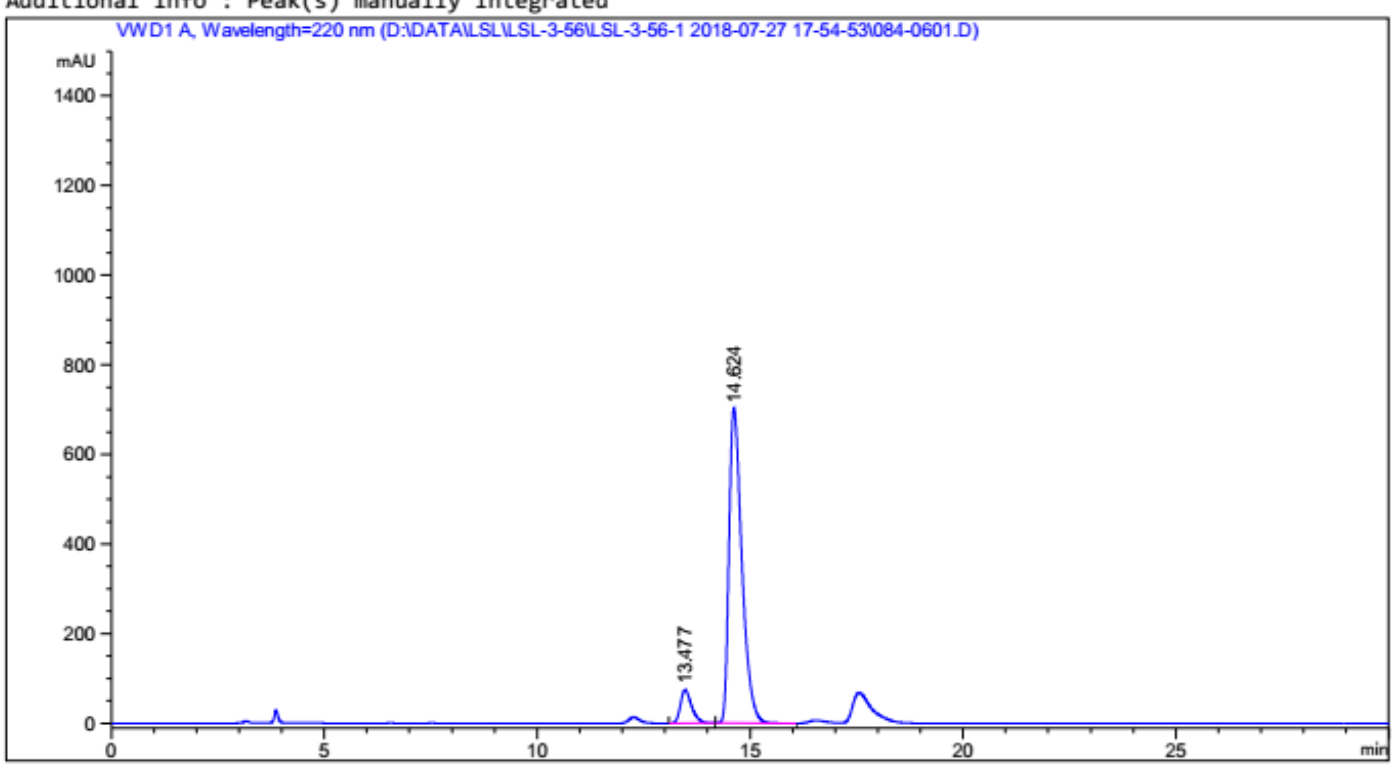

Area Percent Report

Sorted By : : Signal

Multiplier $\quad$ : 1.0000

1.0000

Use Multiplier \& Dilution Factor with ISTDs

Signal 1: VWD1 A, Wavelength $=220 \mathrm{~nm}$

\begin{tabular}{|c|c|c|c|c|c|}
\hline $\begin{array}{c}\text { Peak } \\
\#\end{array}$ & $\begin{array}{l}\text { RetTime Type } \\
\text { [min] }\end{array}$ & $\begin{array}{l}\text { Width } \\
\text { [min] }\end{array}$ & $\begin{array}{c}\text { Area } \\
{\left[\mathrm{mAU}{ }^{*} \mathrm{~s}\right]}\end{array}$ & $\begin{array}{l}\text { Height } \\
\text { [mAU] }\end{array}$ & $\begin{array}{c}\text { Area } \\
\%\end{array}$ \\
\hline & & & & & \\
\hline 1 & $13.477 \mathrm{BB}$ & 0.2751 & 1353.31421 & 74.44087 & 8.3966 \\
\hline
\end{tabular}

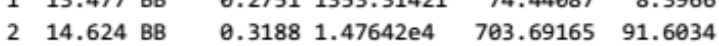

Totals : $\quad 1.61175 \mathrm{e} 4 \quad 778.13252$ 
<smiles>COc1ccc([C@H]2CC(=O)N(C)C2)cc1</smiles>

Data File D: \DATA\LWD\LW-75\W-75 2018-07-28 17-42-31\001-1301.D Sample Name: LSL-3-56-4

\begin{tabular}{|c|c|c|}
\hline Acq. Operator & : & Seq. Line : \\
\hline Acq. Instrument & : Instrument 2 & Location : Vial 1 \\
\hline Injection Date & : 7/29/2018 1:10:43 AM & $\begin{array}{rc}\text { Inj }: & 1 \\
\text { Inj Volume } & : 5.000\end{array}$ \\
\hline
\end{tabular}

Acq. Method : D: \DATA \LWD $L W-75 \backslash W-75$ 2018-07-28 17-42-31 \DAD-AS(1-6)-90-10-1ML-5UL-ALLTOMIN.M

Last changed : 7/28/2018 7:53:41 PM

Analysis Method : D: \METHOD \HZY \DAD-OD(1-2)-90-10-1ML-2UL-ALL-40MIN.M

Last changed : 9/25/2018 10:46:31 AM

(modified after loading)

Additional Info : Peak(s) manually integrated

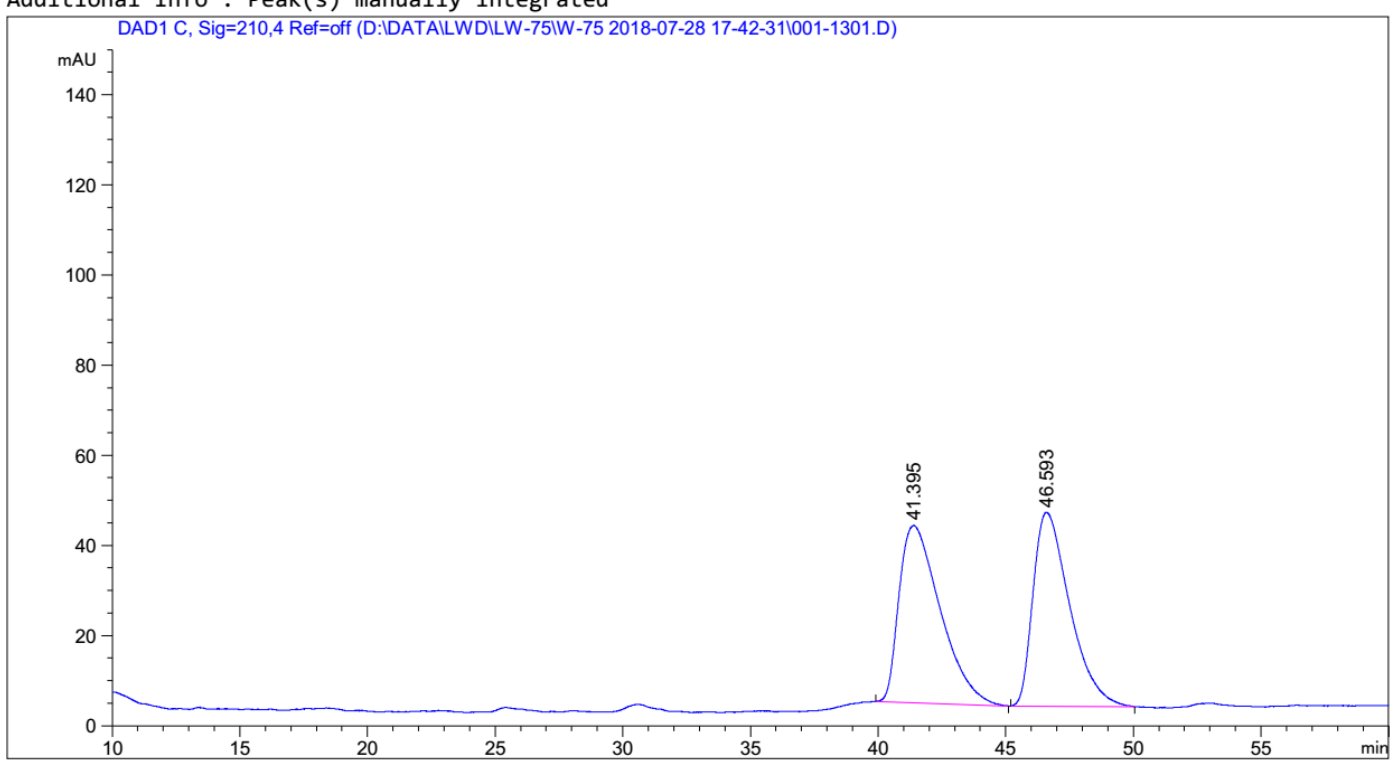

Area Percent Report

\begin{tabular}{|c|c|c|}
\hline Sorted By & $:$ & Signal \\
\hline Multiplier & : & 1.0000 \\
\hline Dilution & : & 1.0000 \\
\hline
\end{tabular}

Use Multiplier \& Dilution Factor with ISTDs

Signal 1: DAD1 C, Sig=210,4 Ref=off

\begin{tabular}{|c|c|c|c|c|c|}
\hline $\begin{array}{c}\text { Peak } \\
\#\end{array}$ & $\begin{array}{l}\text { RetTime Type } \\
\text { [min] }\end{array}$ & $\begin{array}{l}\text { Width } \\
\text { [min] }\end{array}$ & $\begin{array}{c}\text { Area } \\
{[\mathrm{mAU} * \mathrm{~s}]}\end{array}$ & $\begin{array}{l}\text { Height } \\
\text { [mAU] }\end{array}$ & $\begin{array}{c}\text { Area } \\
\quad \%\end{array}$ \\
\hline--- & |---- & 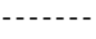 & | - & | & $-\cdots$ \\
\hline 1 & 41.395 BВ & 1.3251 & 4421.09766 & 39.34147 & 50.7435 \\
\hline 2 & 46.593 ВВ & 1.2630 & 4291.54297 & 43.05047 & 49.2565 \\
\hline Tota] & ls : & & 8712.64063 & 82.39194 & \\
\hline
\end{tabular}


Data File D: \DATA \LWD\LW-75\W-75 2018-07-28 17-42-31\002-1401.D Sample Name: LSL-3-40-4

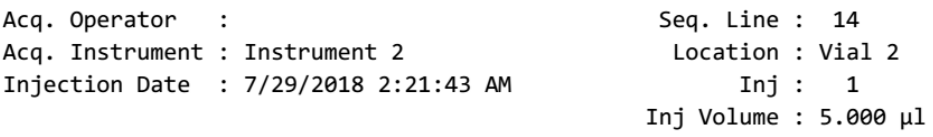

Acq. Method : D: \DATA \LW \LW-75\W-75 2018-07-28 17-42-31\DAD-AS(1-6)-90-10-1ML-5UL-ALL7OMIN.M

Last changed : 7/28/2018 7:53:41 PM

Analysis Method : D: \METHOD \HZY \DAD-OD(1-2)-90-10-1ML-2UL-ALL-40MIN.M

Last changed : 9/25/2018 10:49:09 AM (modified after loading)

Additional Info : Peak(s) manually integrated

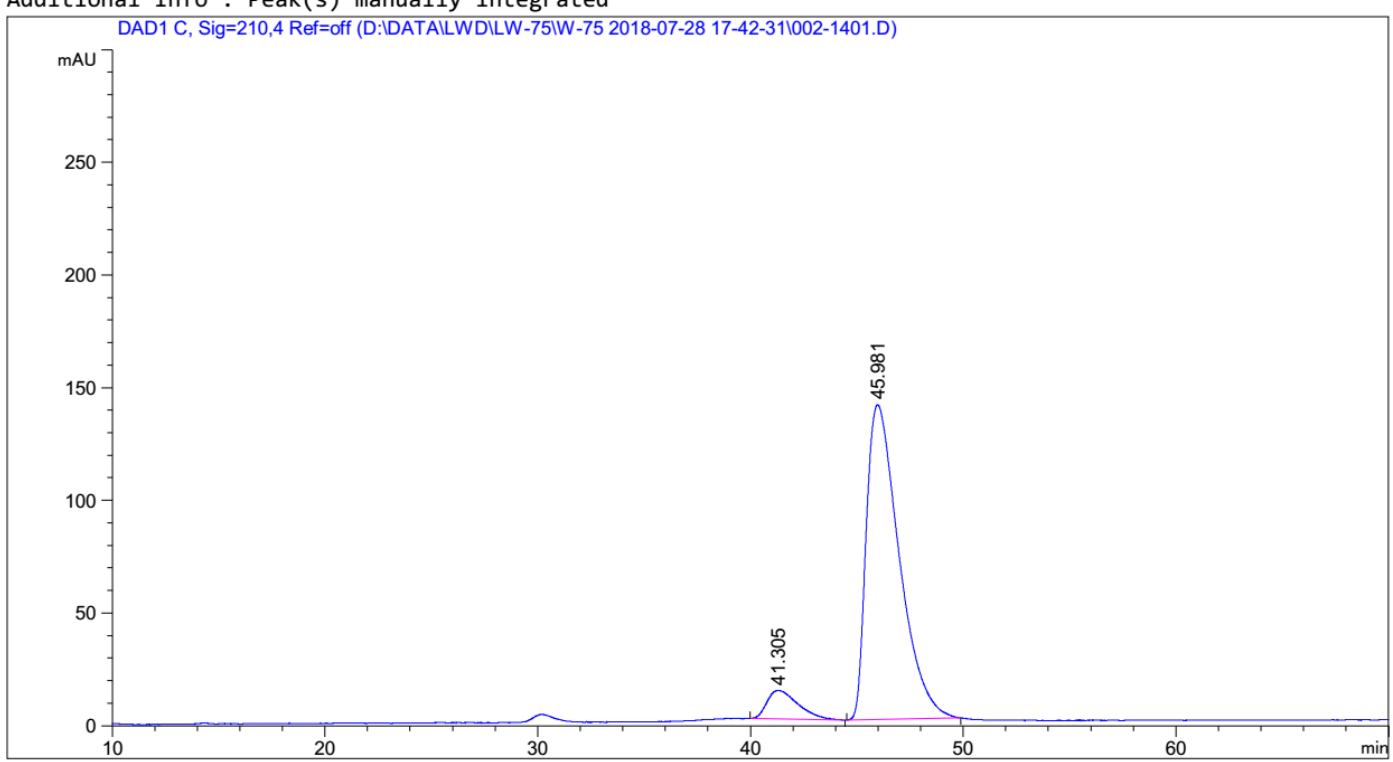

Area Percent Report

$\begin{array}{lll}\text { Sorted By } & : & \text { Signal } \\ \text { Multiplier } & : & 1.0000\end{array}$

Dilution : 1.0000

Use Multiplier \& Dilution Factor with ISTDs

Signal 1: DAD1 C, Sig=210,4 Ref=off

\begin{tabular}{|c|c|c|c|c|c|}
\hline $\begin{array}{c}\text { Peak } \\
\#\end{array}$ & $\begin{array}{l}\text { RetTime Type } \\
\text { [min] }\end{array}$ & $\begin{array}{l}\text { Width } \\
\text { [min] }\end{array}$ & $\begin{array}{c}\text { Area } \\
{\left[\mathrm{mAU}^{*} \mathrm{~s}\right]}\end{array}$ & $\begin{array}{l}\text { Height } \\
{[\mathrm{mAU}]}\end{array}$ & $\begin{array}{c}\text { Area } \\
\%\end{array}$ \\
\hline$-\ldots$ & $-\cdots$ & (n) & |-..-. & - & $\mid$ \\
\hline 1 & $41.305 \mathrm{BB}$ & 1.1913 & 1287.63281 & 12.67349 & 7.9149 \\
\hline 2 & 45.981 BB & 1.4889 & $1.49809 \mathrm{e} 4$ & 139.71852 & 92.0851 \\
\hline Tota & . & & $1.62686 \mathrm{e} 4$ & 152.39201 & \\
\hline
\end{tabular}


<smiles>CN1CC(c2ccc(C(F)(F)F)cc2)CC1=O</smiles>

Data File D: \DATA \LSL\LSL-3-6061\LSL-3-6061 2018-07-31 16-57-31\045-1201.D Sample Name: LSL-3-60-3

\begin{tabular}{|c|c|c|}
\hline Acq. Operator & : & Seq. Line : \\
\hline Acq. Instrument & : Instrument 1 & Location : Vial 45 \\
\hline Injection Date & : 8/1/2018 12:48:46 AM & 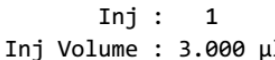 \\
\hline
\end{tabular}

Acq. Method : D: \DATA \LSL\LSL-3-6061\LSL-3-6061 2018-07-31 16-57-31 \VWD-AD(1-2)-95-5-0. 5ML-3UL-220NM-60MIN.M

Last changed : 7/23/2018 8:16:17 AM

Analysis Method : D: \METHOD \LWD \VWD-AD(1-2)-80-20-1ML-3UL-210NM-60MIN.M

Last changed : 9/25/2018 10:24:30 AM

(modified after loading)

Additional Info : Peak(s) manually integrated

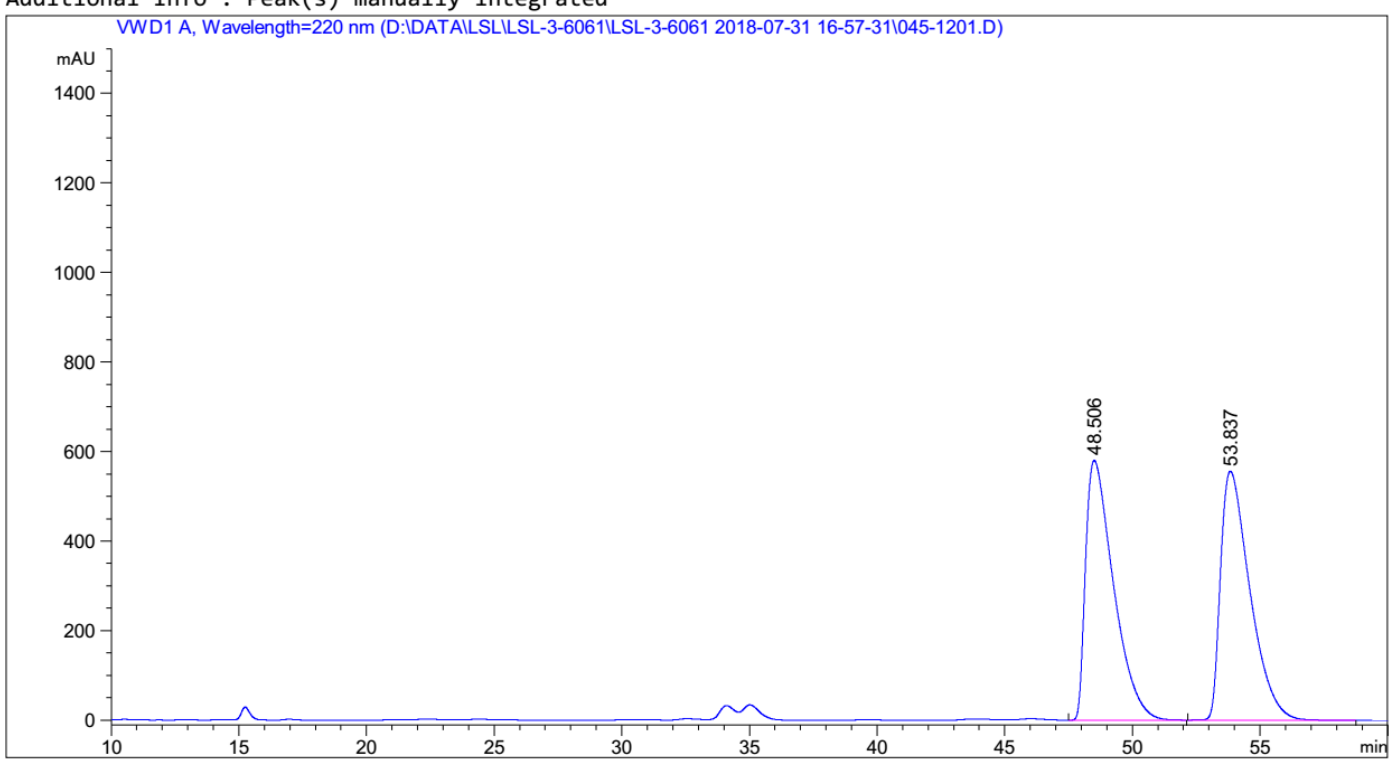

Area Percent Report

$\begin{array}{lll} & & \text { Area Percen } \\ ============================== \\ \text { Sorted By } & : & \text { Signal } \\ \text { Multiplier } & : & 1.0000 \\ \text { Dilution } & : & 1.0000\end{array}$

Use Multiplier \& Dilution Factor with ISTDs

Signal 1: VWD1 A, Wavelength $=220 \mathrm{~nm}$

\begin{tabular}{|c|c|c|c|c|c|c|}
\hline $\begin{array}{c}\text { Peak } \\
\#\end{array}$ & $\begin{array}{c}\text { RetTime } \\
\text { [min] }\end{array}$ & Type & $\begin{array}{l}\text { Width } \\
\text { [min] }\end{array}$ & $\begin{array}{c}\text { Area } \\
{[\mathrm{mAU} * \mathrm{~s}]}\end{array}$ & $\begin{array}{l}\text { Height } \\
{[\mathrm{mAU}]}\end{array}$ & $\begin{array}{c}\text { Area } \\
\%\end{array}$ \\
\hline$-\ldots$ & $-\ldots$ & & $-\ldots$ & | - - & 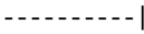 & | \\
\hline 1 & 48.506 & BB & 1.1413 & $4.41201 \mathrm{e} 4$ & 580.08295 & 49.8107 \\
\hline 2 & 53.837 & BB & 1.2090 & $4.44554 \mathrm{e} 4$ & 555.89447 & 50.1893 \\
\hline Tot & : & & & $8.85755 \mathrm{e} 4$ & 1135.97742 & \\
\hline
\end{tabular}


Data File D: \DATA \LSL\LSL-3-6061\LSL-3-6061 2018-07-31 16-57-31\046-1301.D Sample Name: LSL-3-40-2

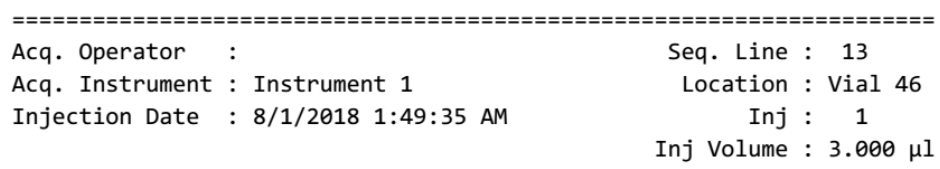

Acq. Method : D: \DATA \LSL\LSL-3-6061\LSL-3-6061 2018-07-31 16-57-31 \VWD-AD(1-2)-95-5-0. 5ML-3UL-220NM-60MIN.M

Last changed $\quad$ : 7/23/2018 8:16:17 AM

Analysis Method: D: \METHOD \LWD \VWD-AD(1-2)-80-20-1ML-3UL-210NM-60MIN.M

Last changed : 9/25/2018 10:26:40 AM

(modified after loading)

Additional Info : Peak(s) manually integrated

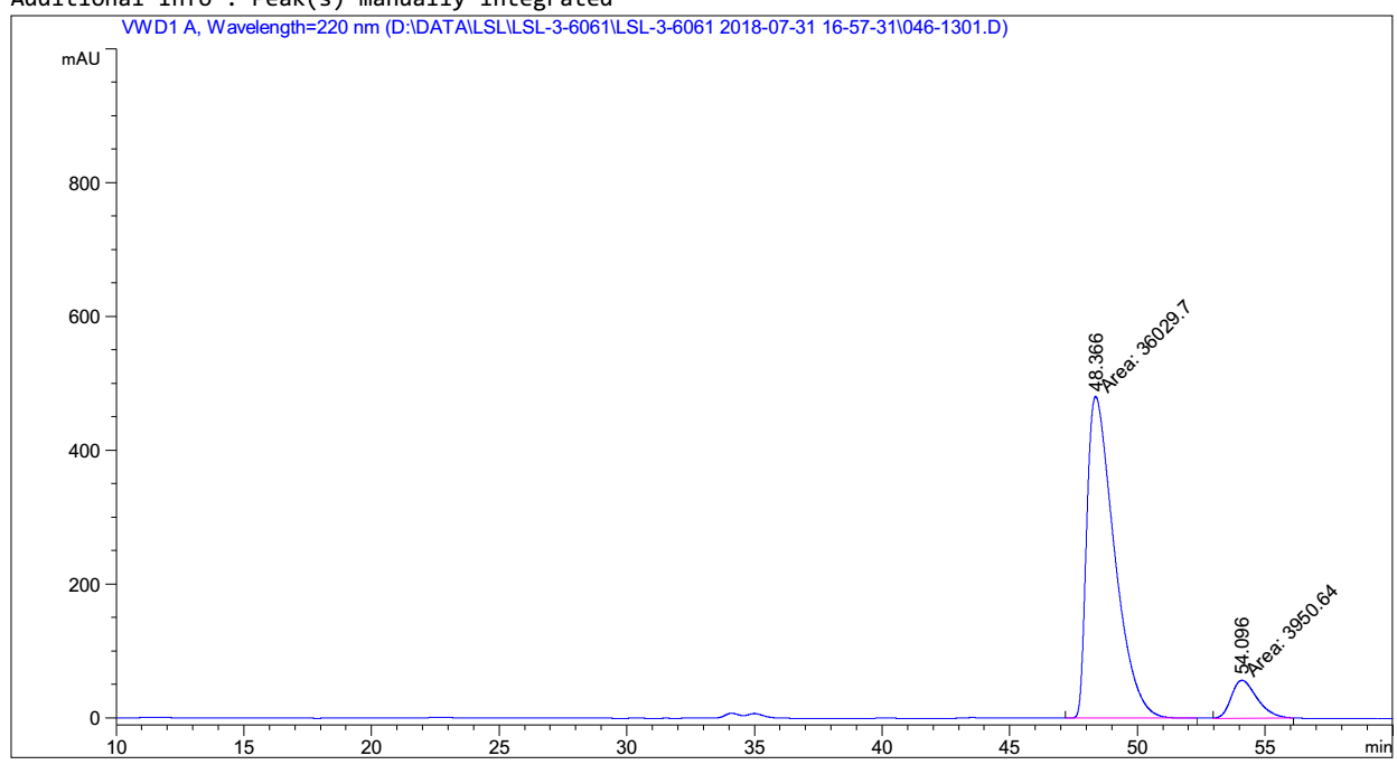

Area Percent Report

$\begin{array}{lll}\text { Sorted By } & : & \text { Signal } \\ \text { Multiplier } & : & 1.0000 \\ \text { Dilution } & : & 1.0000\end{array}$

Use Multiplier \& Dilution Factor with ISTDs

Signal 1: VWD1 A, Wavelength $=220 \mathrm{~nm}$

\begin{tabular}{|c|c|c|c|c|c|c|}
\hline $\begin{array}{c}\text { Peak } \\
\#\end{array}$ & $\begin{array}{c}\text { RetTime } \\
\text { [min] }\end{array}$ & Type & $\begin{array}{l}\text { Width } \\
\text { [min] }\end{array}$ & $\begin{array}{c}\text { Area } \\
{\left[\mathrm{mAU}^{*} \mathrm{~s}\right]}\end{array}$ & $\begin{array}{l}\text { Height } \\
\text { [mAU] }\end{array}$ & $\begin{array}{c}\text { Area } \\
\quad \%\end{array}$ \\
\hline- & & & & |-....... & | & | \\
\hline 1 & 48.366 & MM & 1.2490 & $3.60297 \mathrm{e} 4$ & 480.76645 & 90.1185 \\
\hline 2 & 54.096 & MM & 1.1611 & 3950.64478 & 56.70885 & 9.8815 \\
\hline Tota & Is : & & & $3.99804 \mathrm{e} 4$ & 537.47530 & \\
\hline
\end{tabular}


<smiles>CN1C[C@H](c2ccc(F)cc2)CC1=O</smiles>

Data File D: \DATA\LSL\LSL-3-56\LSL-3-56-1 2018-07-27 17-54-53\085-0701.D Sample Name: LSL-3-56-3

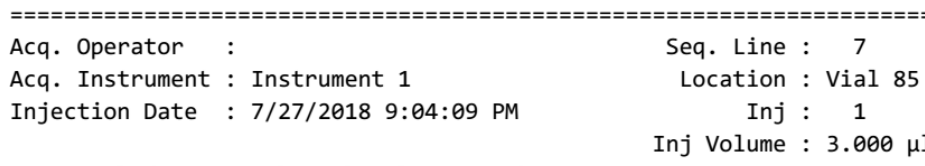

Acq. Method : D: \DATA \LSL\LSL-3-56\LSL-3-56-1 2018-07-27 17-54-53 VWD-AD(1-2)-95-5-1ML3UL-220NM-40MIN.M

Last changed : 6/7/2018 11:25:33 AM

Analysis Method : D: \METHOD\LWD \VWD-AD(1-2)-80-20-1ML-3UL-210NM-60MIN.M

Last changed : 9/25/2018 10:37:31 AM

(modified after loading)

Additional Info : Peak(s) manually integrated

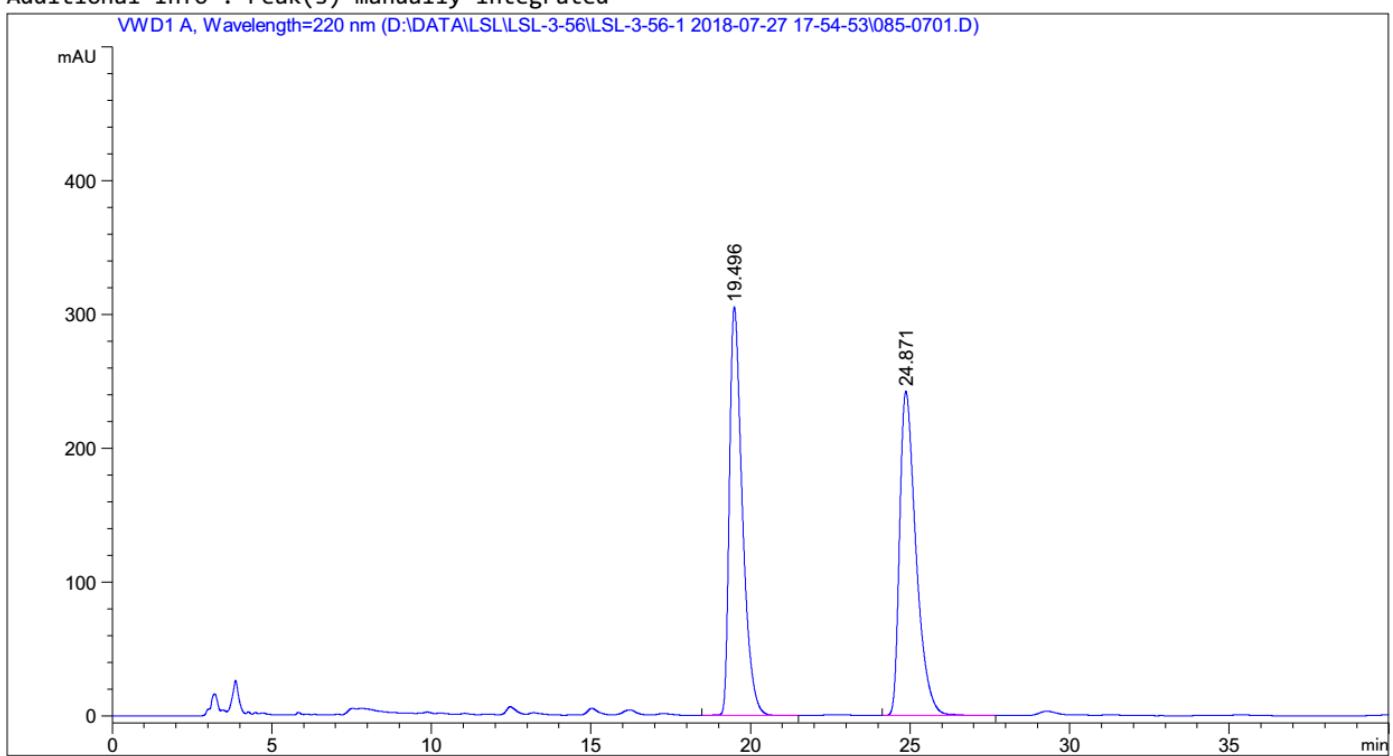

Area Percent Report

$\begin{array}{lll}\text { Sorted By } & : & \text { Signal } \\ \text { Multiplier } & : & 1.0000 \\ \text { Dilution } & : & 1.0000\end{array}$

Use Multiplier \& Dilution Factor with ISTDs

Signal 1: VWD1 A, Wavelength $=220 \mathrm{~nm}$

\begin{tabular}{|c|c|c|c|c|c|}
\hline $\begin{array}{c}\text { Peak } \\
\#\end{array}$ & $\begin{array}{l}\text { RetTime Type } \\
\text { [min] }\end{array}$ & $\begin{array}{l}\text { Width } \\
\text { [min] }\end{array}$ & $\begin{array}{c}\text { Area } \\
{\left[\mathrm{mAU}^{*} \mathrm{~s}\right]}\end{array}$ & $\begin{array}{l}\text { Height } \\
\text { [mAU] }\end{array}$ & $\begin{array}{c}\text { Area } \\
\%\end{array}$ \\
\hline.-- & $|-\ldots-\ldots|-$ & & | & | & | \\
\hline 1 & $19.496 \mathrm{BB}$ & 0.4260 & 8584.62500 & 305.33511 & 49.8685 \\
\hline 2 & 24.871 BB & 0.5410 & 8629.90918 & 242.00035 & 50.1315 \\
\hline
\end{tabular}

Totals : $\quad 1.72145 \mathrm{e} 4 \quad 547.33546$ 
Data File D: \DATA \LSL\LSL-3-56\LSL-3-56-1 2018-07-27 17-54-53\086-0801.D Sample Name: LSL-3-46-3

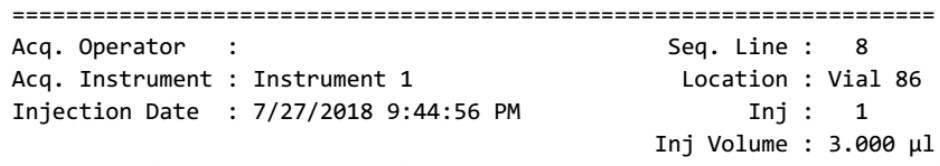

Acq. Method : D: \DATA \LSL \LSL-3-56\LSL-3-56-1 2018-07-27 17-54-53\VWD-AD(1-2)-95-5-1ML3UL-220NM-40MIN.M

Last changed : 6/7/2018 11:25:33 AM

Analysis Method : D: \METHOD \LWD \VWD-AD(1-2)-80-20-1ML-3UL-210NM-60MIN.M

Last changed : 9/25/2018 10:38:49 AM

(modified after loading)

Additional Info : Peak(s) manually integrated

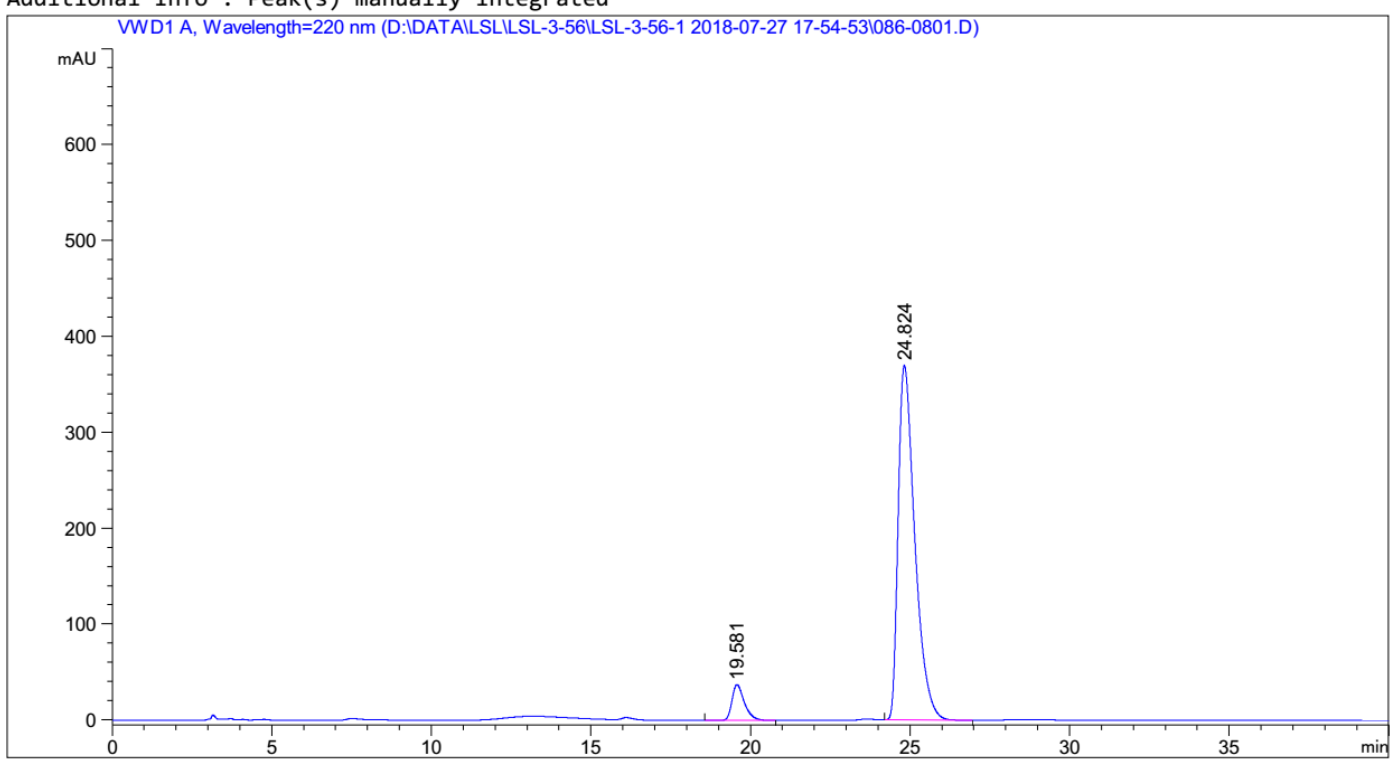

Area Percent Report

$\begin{array}{lll}\text { Sorted By } & : & \text { Signal } \\ \text { Multiplier } & : & 1.0000 \\ \text { Dilution } & : & 1.0000\end{array}$

Use Multiplier \& Dilution Factor with ISTDs

Signal 1: VWD1 A, Wavelength $=220 \mathrm{~nm}$

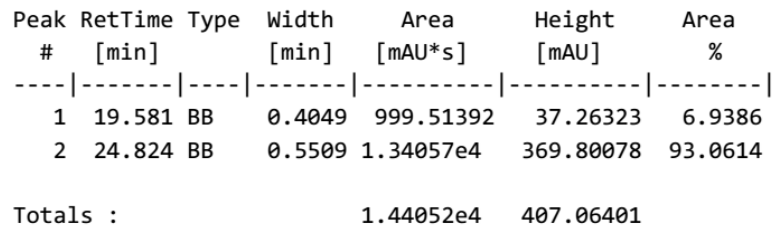


<smiles>CN1C[C@H](c2ccc(Cl)cc2)CC1=O</smiles>

Data File D: \DATA \LSL\LSL-3-6061\LSL-3-6061 2018-07-31 16-57-31\043-1001.D Sample Name: LSL-3-60-2

\begin{tabular}{|c|c|c|}
\hline Acq. Operator & : & Seq. Line : 10 \\
\hline Acq. Instrument & : Instrument 1 & Location : Vial 43 \\
\hline Injection Date & : 7/31/2018 10:47:05 PM & $\begin{array}{rlc}\text { Inj } & : & 1 \\
\text { Inj Volume } & : & 3.000 \mu\end{array}$ \\
\hline
\end{tabular}

Acq. Method : D: \DATA \LSL\LSL-3-6061\LSL-3-6061 2018-07-31 16-57-31 \VWD-AD(1-2)-95-5-0. 5ML-3UL-220NM-60MIN.M

Last changed : 7/23/2018 8:16:17 AM

Analysis Method : D: \METHOD\LWD \VWD-AD(1-2)-80-20-1ML-3UL-210NM-60MIN.M

Last changed : 9/25/2018 10:20:13 AM

(modified after loading)

Additional Info : Peak(s) manually integrated

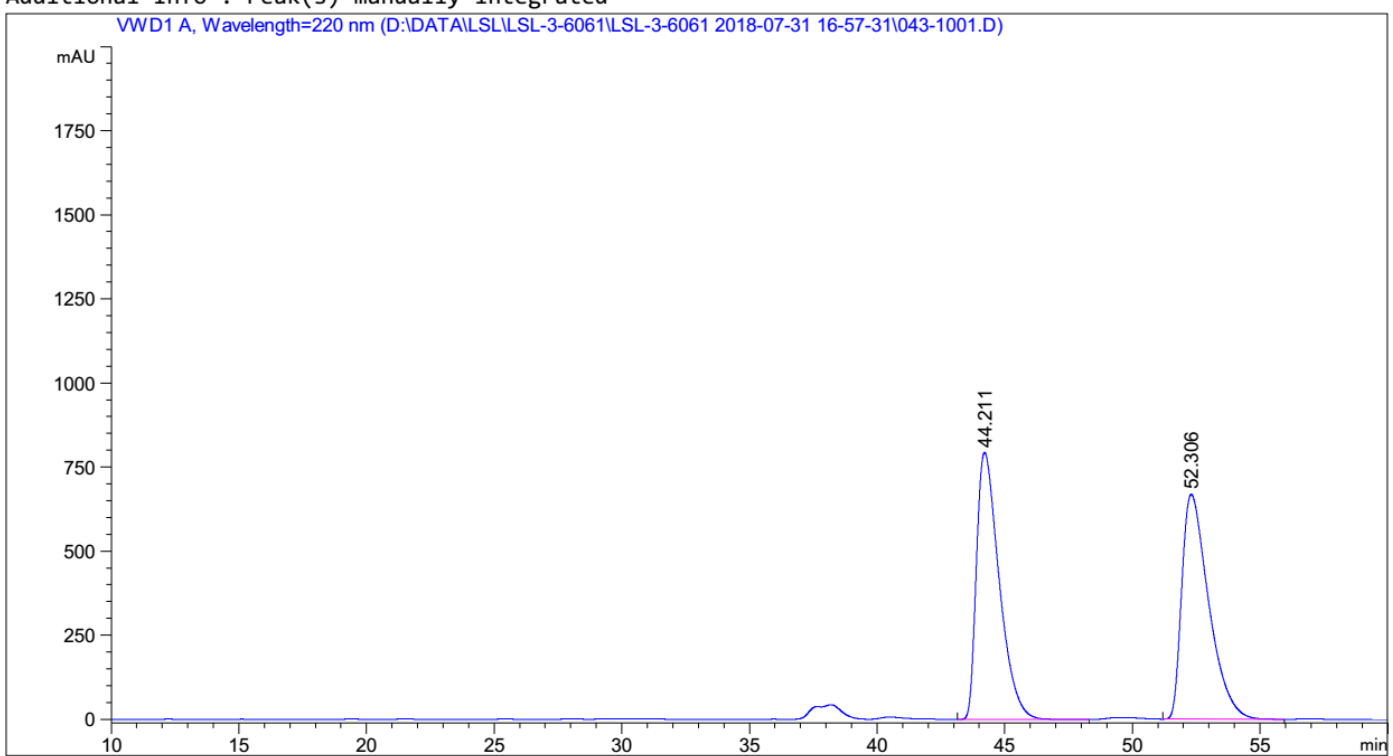

Area Percent Report

$\begin{array}{lll} & & \text { Area Percen } \\ ============================== \\ \text { Sorted By } & : & \text { Signal } \\ \text { Multiplier } & : & 1.0000 \\ \text { Dilution } & : & 1.0000\end{array}$

Use Multiplier \& Dilution Factor with ISTDs

Signal 1: VWD1 A, Wavelength $=220 \mathrm{~nm}$

\begin{tabular}{|c|c|c|c|c|c|c|}
\hline $\begin{array}{c}\text { Peak } \\
\#\end{array}$ & $\begin{array}{c}\text { RetTime } \\
\text { [min] }\end{array}$ & Type & $\begin{array}{l}\text { Width } \\
\text { [min] }\end{array}$ & $\begin{array}{c}\text { Area } \\
{\left[\mathrm{mAU}^{*} \mathrm{~s}\right]}\end{array}$ & $\begin{array}{l}\text { Height } \\
{[\mathrm{mAU}]}\end{array}$ & $\begin{array}{c}\text { Area } \\
\%\end{array}$ \\
\hline$-\ldots$ & -.... & & - & | - & | & | \\
\hline 1 & 44.211 & BB & 0.9394 & $4.90218 \mathrm{e} 4$ & 793.86389 & 49.9627 \\
\hline 2 & 52.306 & BB & 1.1124 & $4.90950 \mathrm{e} 4$ & 668.84534 & 50.0373 \\
\hline Tot & : & & & $9.81168 \mathrm{e} 4$ & 1462.70923 & \\
\hline
\end{tabular}


Data File D: \DATA \LSL\LSL-3-6061\LSL-3-6061 2018-07-31 16-57-31\044-1101.D Sample Name: LSL-3-46-4

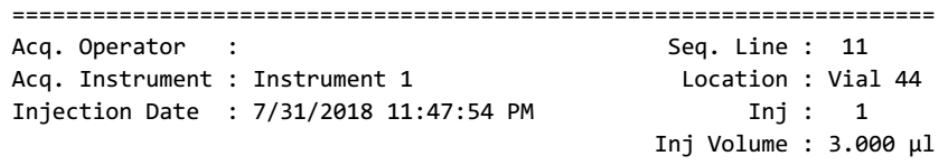

Acq. Method : D: \DATA \LSL\LSL-3-6061\LSL-3-6061 2018-07-31 16-57-31 \WD-AD(1-2)-95-5-0. 5ML-3UL-220NM-60MIN.M

Last changed : 7/23/2018 8:16:17 AM

Analysis Method: D: \METHOD\LWD \VWD-AD(1-2)-80-20-1ML-3UL-210NM-60MIN.M

Last changed : 9/25/2018 10:22:45 AM

(modified after loading)

Additional Info : Peak(s) manually integrated

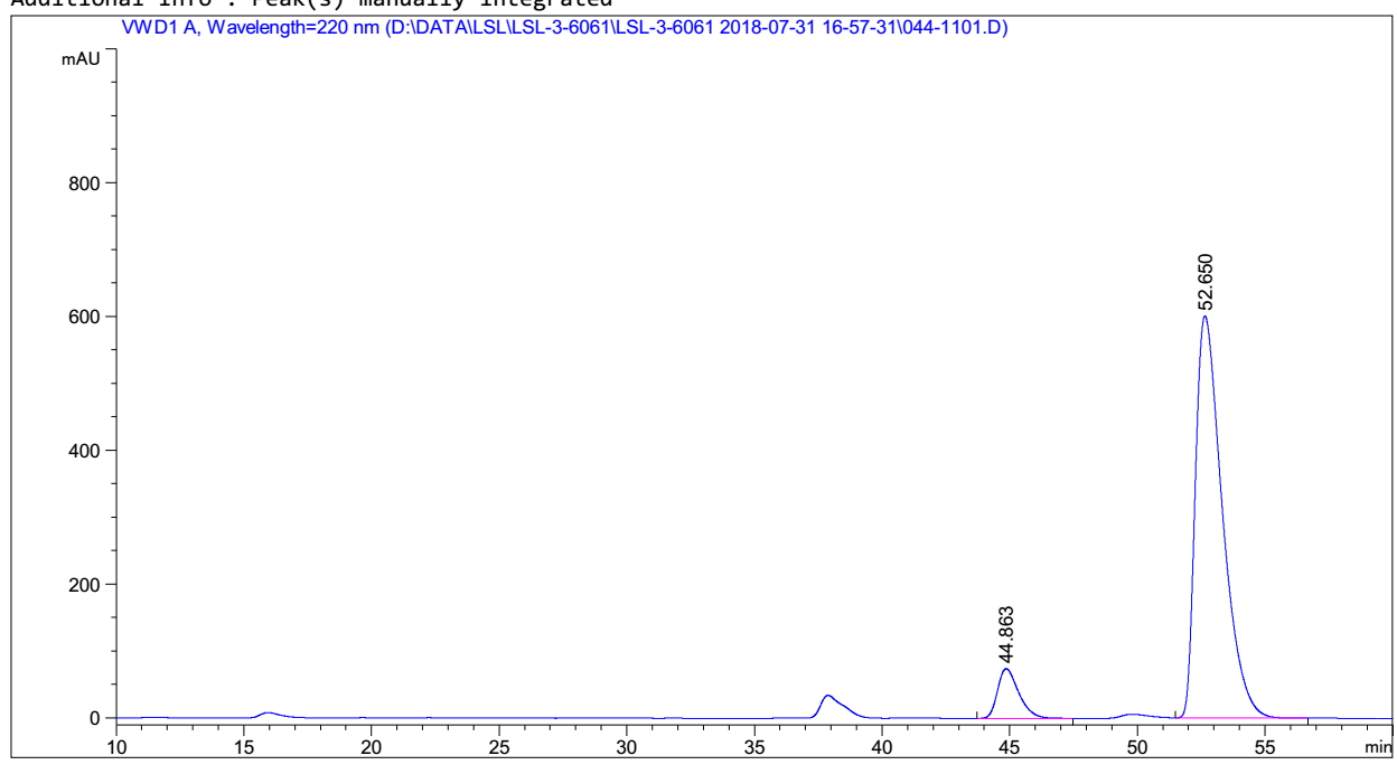

Area Percent Report

$\begin{array}{lll}\text { Sorted By } & : & \text { Signal } \\ \text { Multiplier } & : & 1.0000 \\ \text { Dilution } & : & 1.0000\end{array}$

Use Multiplier \& Dilution Factor with ISTDs

Signal 1: VWD1 A, Wavelength $=220 \mathrm{~nm}$

\begin{tabular}{|c|c|c|c|c|c|c|}
\hline $\begin{array}{c}\text { Peak } \\
\#\end{array}$ & $\begin{array}{c}\text { RetTime } \\
\text { [min] }\end{array}$ & Type & $\begin{array}{l}\text { Width } \\
\text { [min] }\end{array}$ & $\begin{array}{c}\text { Area } \\
{\left[\mathrm{mAU}^{*} \mathrm{~s}\right]}\end{array}$ & $\begin{array}{l}\text { Height } \\
{[\mathrm{mAU}]}\end{array}$ & $\begin{array}{c}\text { Area } \\
\%\end{array}$ \\
\hline$=-$ & $-\ldots$ & & & | - - & - & - - - \\
\hline 1 & 44.863 & BB & 0.8702 & 4256.79541 & 74.21692 & 8.8214 \\
\hline 2 & 52.650 & BB & 1.1104 & $4.39984 \mathrm{e} 4$ & 600.78113 & 91.1786 \\
\hline Tota. & : & & & $4.82552 \mathrm{e} 4$ & 674.99805 & \\
\hline
\end{tabular}


<smiles>Cc1cc(C)cc([C@H]2CCOC2)c1</smiles>

6a

Data File D: \DATA \LSL\LSL-3-85\LSL-3-85 2018-09-01 20-44-06\011-0301.D Sample Name: LSL-3-79

\begin{tabular}{|c|c|c|}
\hline Acq. Operator & : & Seq. Line : 3 \\
\hline Acq. Instrument & : Instrument 1 & Location : Vial 11 \\
\hline Injection Date & : 9/1/2018 9:06:43 PM & 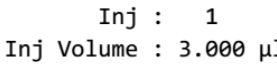 \\
\hline
\end{tabular}

Acq. Method : D: \DATA \LSL\LSL-3-85\LSL-3-85 2018-09-01 20-44-06\VWD-AS(1-6)-99-1-0.3ML3UL-220NM-40MIN.M

Last changed : 8/27/2018 7:39:23 PM

Analysis Method : D: \METHOD \LWD \VWD-AD(1-2)-80-20-1ML-3UL-210NM-60MIN.M

Last changed : 9/25/2018 11:10:54 AM

(modified after loading)

Additional Info : Peak(s) manually integrated

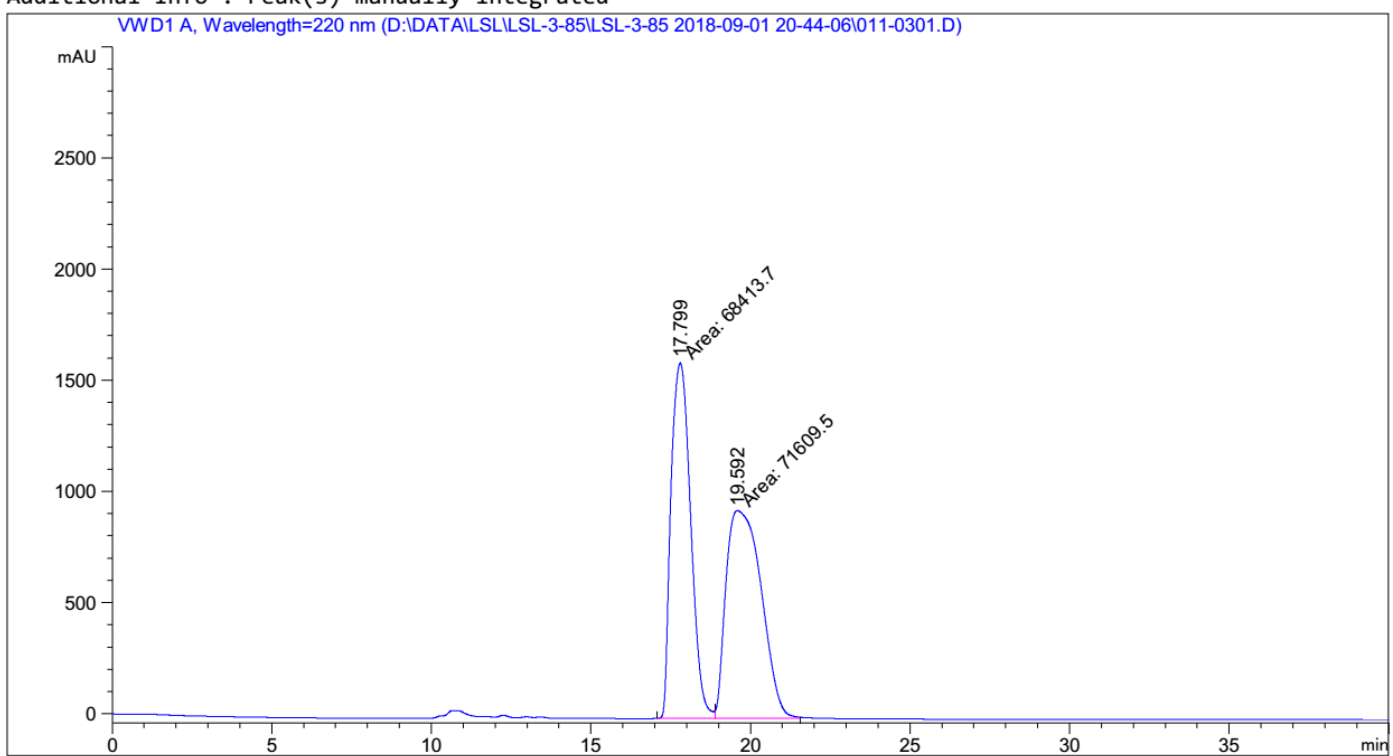

Area Percent Report

Sorted By : : Signal

Multiplier $\quad$ : 1.0000

Dilution : 1.0000

Use Multiplier \& Dilution Factor with ISTDs

Signal 1: VWD1 A, Wavelength $=220 \mathrm{~nm}$

\begin{tabular}{|c|c|c|c|c|c|c|}
\hline $\begin{array}{c}\text { Peak } \\
\#\end{array}$ & $\begin{array}{c}\text { RetTime } \\
\text { [min] }\end{array}$ & Type & $\begin{array}{l}\text { Width } \\
\text { [min] }\end{array}$ & $\begin{array}{c}\text { Area } \\
{[\mathrm{mAU} * \mathrm{~s}]}\end{array}$ & $\begin{array}{l}\text { Height } \\
{[\mathrm{mAU}]}\end{array}$ & $\begin{array}{c}\text { Area } \\
\quad \%\end{array}$ \\
\hline$-\ldots$ & -.... & & - & | - & | & | \\
\hline 1 & 17.799 & MF & 0.7141 & $6.84137 \mathrm{e} 4$ & 1596.71021 & 48.8588 \\
\hline 2 & 19.592 & $\mathrm{FM}$ & 1.2791 & $7.16095 \mathrm{e} 4$ & 933.03967 & 51.1412 \\
\hline Tot & : & & & $1.40023 \mathrm{e} 5$ & 2529.74988 & \\
\hline
\end{tabular}




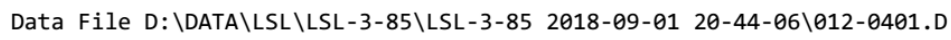
Sample Name: LSL-3-85

\begin{tabular}{|c|c|c|}
\hline Acq. Operator & : & Seq. Line : \\
\hline Acq. Instrument & : Instrument 1 & Location : Vial 12 \\
\hline Injection Date & : 9/1/2018 9:47:33 PM & $\begin{array}{rc}\text { Inj }: & 1 \\
\text { Inj Volume } & 3.000 \mu\end{array}$ \\
\hline
\end{tabular}

Acq. Method : D: \DATA \LSL\LSL-3-85\LSL-3-85 2018-09-01 20-44-06\VWD-AS(1-6)-99-1-0.3ML3UL-220NM-40MIN.M

Last changed : 8/27/2018 7:39:23 PM

Analysis Method: D: \METHOD\LWD \VWD-AD(1-2)-80-20-1ML-3UL-210NM-60MIN.M

Last changed : 9/25/2018 11:08:52 AM

(modified after loading)

Additional Info : Peak(s) manually integrated

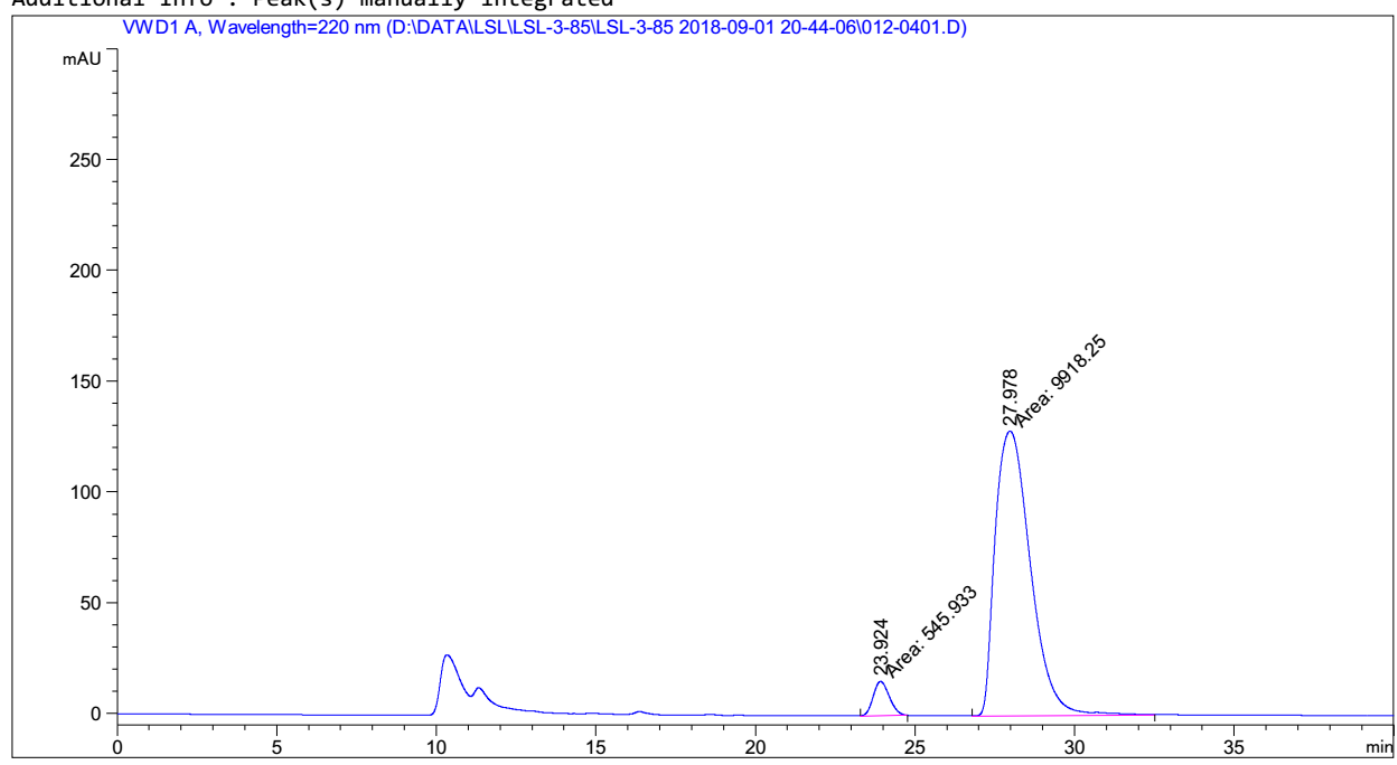

Area Percent Report

$\begin{array}{lll}\text { Sorted By } & : & \text { Signal } \\ \text { Multiplier } & : & 1.0000 \\ \text { Dilution } & : & 1.0000\end{array}$

Use Multiplier \& Dilution Factor with ISTDs

Signal 1: VWD1 A, Wavelength $=220 \mathrm{~nm}$

\begin{tabular}{|c|c|c|c|c|c|c|}
\hline $\begin{array}{c}\text { Peak } \\
\#\end{array}$ & $\begin{array}{c}\text { RetTime } \\
\text { [min] }\end{array}$ & Type & $\begin{array}{l}\text { Width } \\
\text { [min] }\end{array}$ & $\begin{array}{c}\text { Area } \\
{\left[\mathrm{mAU}^{*} \mathrm{~s}\right]}\end{array}$ & $\begin{array}{l}\text { Height } \\
\text { [mAU] }\end{array}$ & $\begin{array}{c}\text { Area } \\
\%\end{array}$ \\
\hline & & & & |- - & 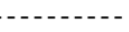 & | \\
\hline 1 & 23.924 & MM & 0.5876 & 545.93280 & 15.48602 & 5.2172 \\
\hline 2 & 27.978 & MM & 1.2860 & 9918.25488 & 128.53702 & 94.7828 \\
\hline Tota & : & & & $1.04642 \mathrm{e} 4$ & 144.02304 & \\
\hline
\end{tabular}




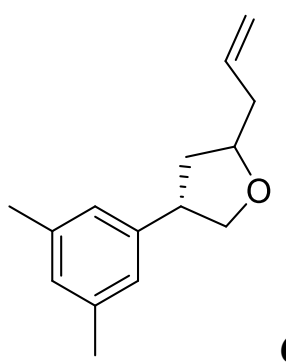

6b

Data File D: \DATA \LSL\LSL-3-68\LSL-3-68 2018-08-11 10-02-28\011-0601.D Sample Name: LSL-3-68-2

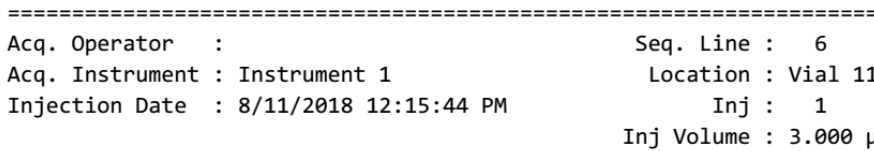

Acq. Method : D: \DATA \LSL\LSL-3-68\LSL-3-68 2018-08-11 10-02-28\VWD-0](1-6)-99-1-0.5ML3UL-220NM-30MIN.M

Last changed : 8/11/2018 11:42:19 AM

Analysis Method : D: \METHOD LWD \VWD-AD(1-2)-80-20-1ML-3UL-210NM-60MIN.M

Last changed : 9/25/2018 11:14:23 AM

(modified after loading)

Additional Info : Peak(s) manually integrated

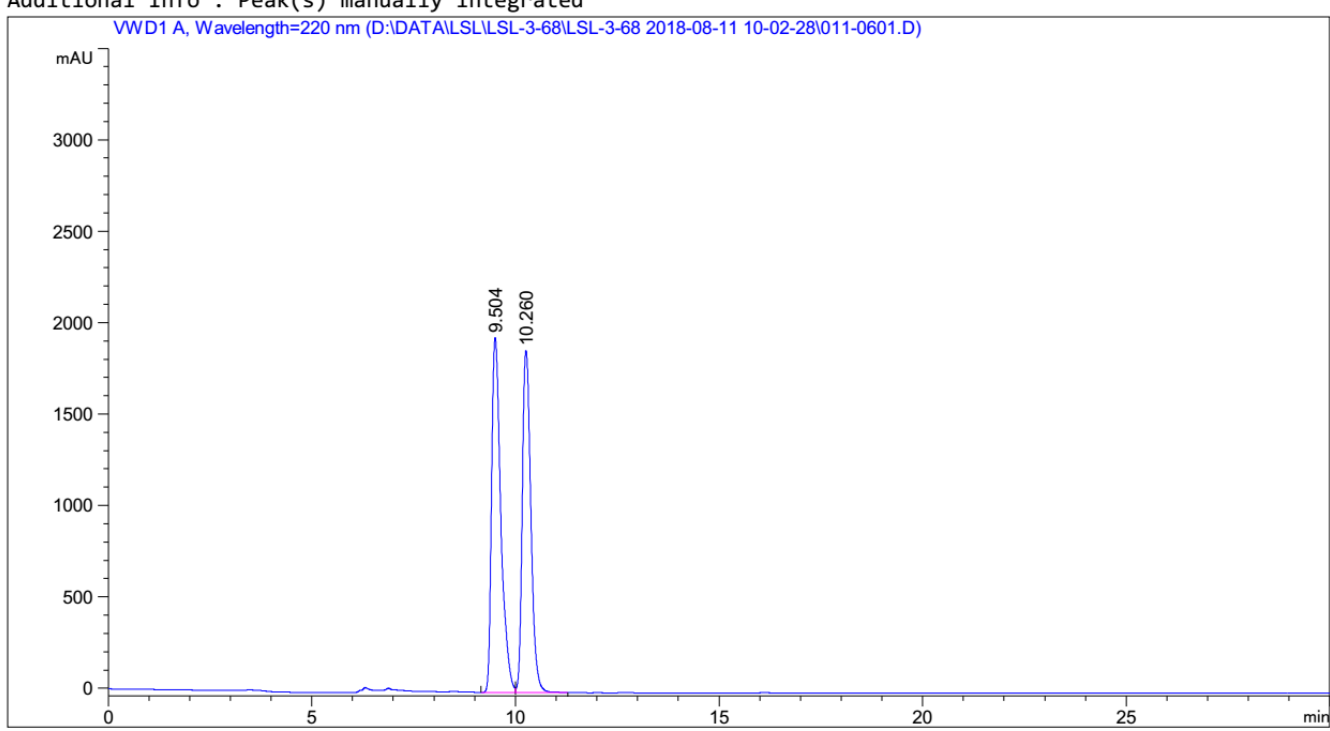

Area Percent Report

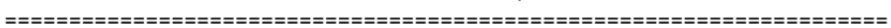

$\begin{array}{lll}\text { Sorted By } & : & \text { Signal } \\ \text { Multiplier } & : & 1.0000 \\ \text { Dilution } & : & 1.0000\end{array}$

Use Multiplier \& Dilution Factor with ISTDs

Signal 1: VWD1 A, Wavelength $=220 \mathrm{~nm}$

\begin{tabular}{|c|c|c|c|c|c|}
\hline $\begin{array}{c}\text { Peak } \\
\#\end{array}$ & $\begin{array}{l}\text { RetTime Type } \\
\text { [min] }\end{array}$ & $\begin{array}{l}\text { Width } \\
\text { [min] }\end{array}$ & $\begin{array}{c}\text { Area } \\
{[\mathrm{mAU} * \mathrm{~s}]}\end{array}$ & $\begin{array}{l}\text { Height } \\
\text { [mAU] }\end{array}$ & $\begin{array}{c}\text { Area } \\
\%\end{array}$ \\
\hline & & & - - & |---.---.-- & - \\
\hline 1 & 9.504 BV & 0.2408 & $3.07208 \mathrm{e} 4$ & 1939.86926 & 52.7723 \\
\hline 2 & $10.260 \mathrm{VV}$ & 0.2296 & $2.74931 \mathrm{e} 4$ & 1869.69397 & 47.2277 \\
\hline Tota & & & $5.82139 \mathrm{e} 4$ & 3809.56323 & \\
\hline
\end{tabular}


Data File D: \DATA\LSL\LSL-3-68\LSL-3-68-1 2018-08-11 15-39-21\012-0401.D Sample Name: LSL-3-68

Acq. Operator

Acq. Instrument : Instrument 1

Injection Date : 8/11/2018 4:42:48 PM

D: DATAYLSLYLSL-3-68YLSL-3-68-1 $2018-08-11$ 15j Volume : $3.000 \mu 1$ 3UL-220NM-30MIN.M

Last changed : 8/11/2018 11:42:19 AM

Analysis Method : D: \METHOD\LWDIVWD-OD(1-2)-100-0-0.5ML-5UL-254NM-60MIN.M

Last changed : 9/28/2018 9:25:58 PM

(modified after loading)

Additional Info : Peak(s) manually integrated

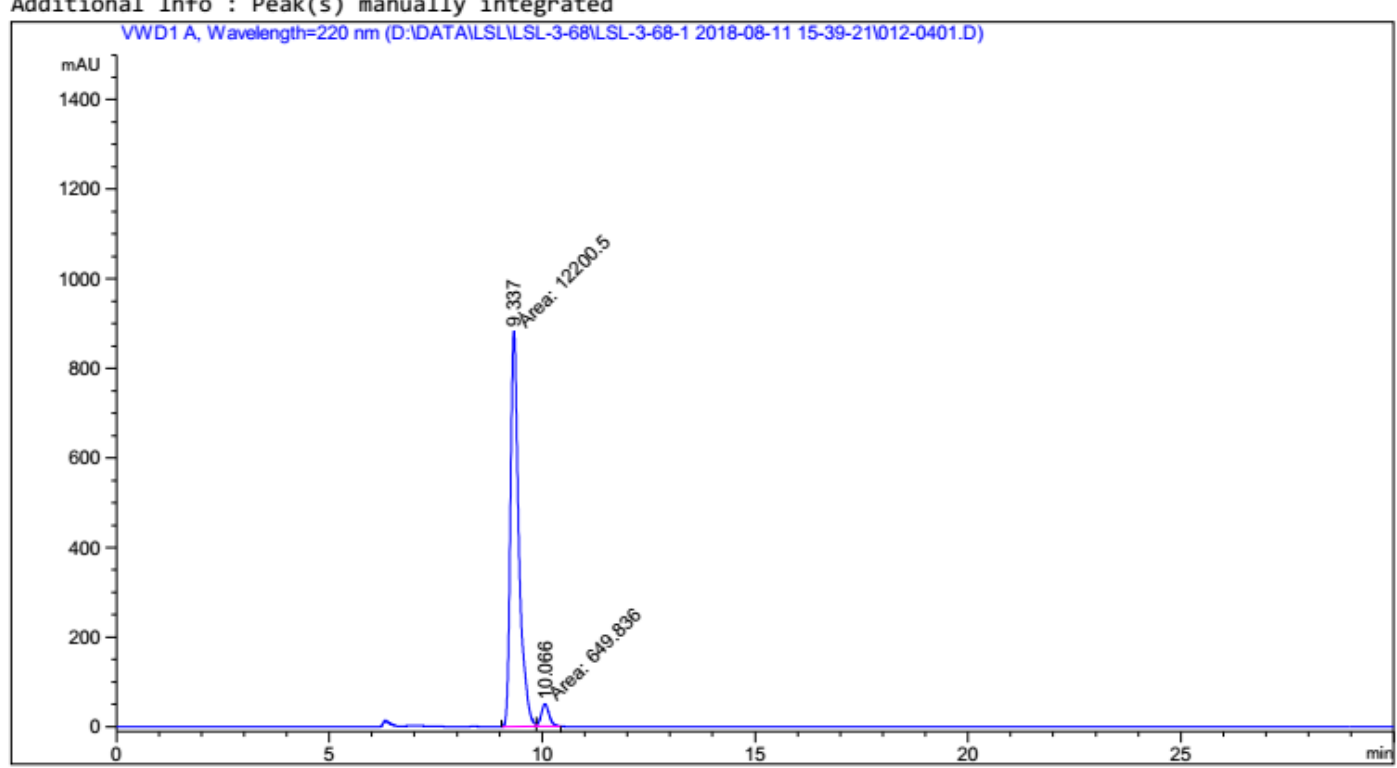

Area Percent Report

Sorted By : : Signal

Multiplier : $\quad 1.0000$

1.0000
1.0000

Use Multiplier \& Dilution Factor with ISTDs

Signal 1: VWD1 A, Wavelength $=220 \mathrm{~nm}$

\begin{tabular}{|c|c|c|c|c|c|}
\hline $\begin{array}{c}\text { Peak } \\
\#\end{array}$ & $\begin{array}{l}\text { RetTime Type } \\
\text { [min] }\end{array}$ & $\begin{array}{l}\text { Width } \\
\text { [min] }\end{array}$ & $\begin{array}{c}\text { Area } \\
{[\mathrm{mAU} * \mathrm{~s}]}\end{array}$ & $\begin{array}{l}\text { Height } \\
\text { [mAU] }\end{array}$ & $\begin{array}{c}\text { Area } \\
\%\end{array}$ \\
\hline - & 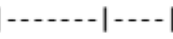 & & 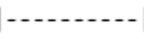 & - & | \\
\hline 1 & $9.337 \mathrm{MF}$ & 0.2304 & $1.22005 \mathrm{e} 4$ & 882.55859 & 94.9430 \\
\hline 2 & $10.066 \mathrm{FM}$ & 0.2152 & 649.83557 & 50.33497 & 5.0570 \\
\hline Tota & & & $1.28503 \mathrm{e} 4$ & 932.89356 & \\
\hline
\end{tabular}


<smiles>Cc1cc(C)cc([C@@H](CO)CCO)c1</smiles>

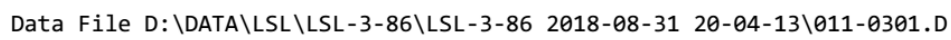
Sample Name: LSL-3-81
Acq. Operator :
Seq. Line : 3
Location : Vial 11
Acq. Instrument : Instrument 1
Inj : 1
Injection Date : 8/31/2018 8:26:50 PM
Inj Volume : $3.000 \mu 1$

Acq. Method : D: \DATA \LSL \LSL-3-86\LSL-3-86 2018-08-31 20-04-13\VWD-AS(1-6)-90-10-1ML-3UL -220NM-60MIN.M

Last changed : 7/20/2018 11:24:22 AM

Analysis Method : D: \METHOD\LWD VWD-AD(1-2)-80-20-1ML-3UL-210NM-60MIN.M

Last changed : 9/25/2018 10:58:43 AM

(modified after loading)

Additional Info : Peak(s) manually integrated

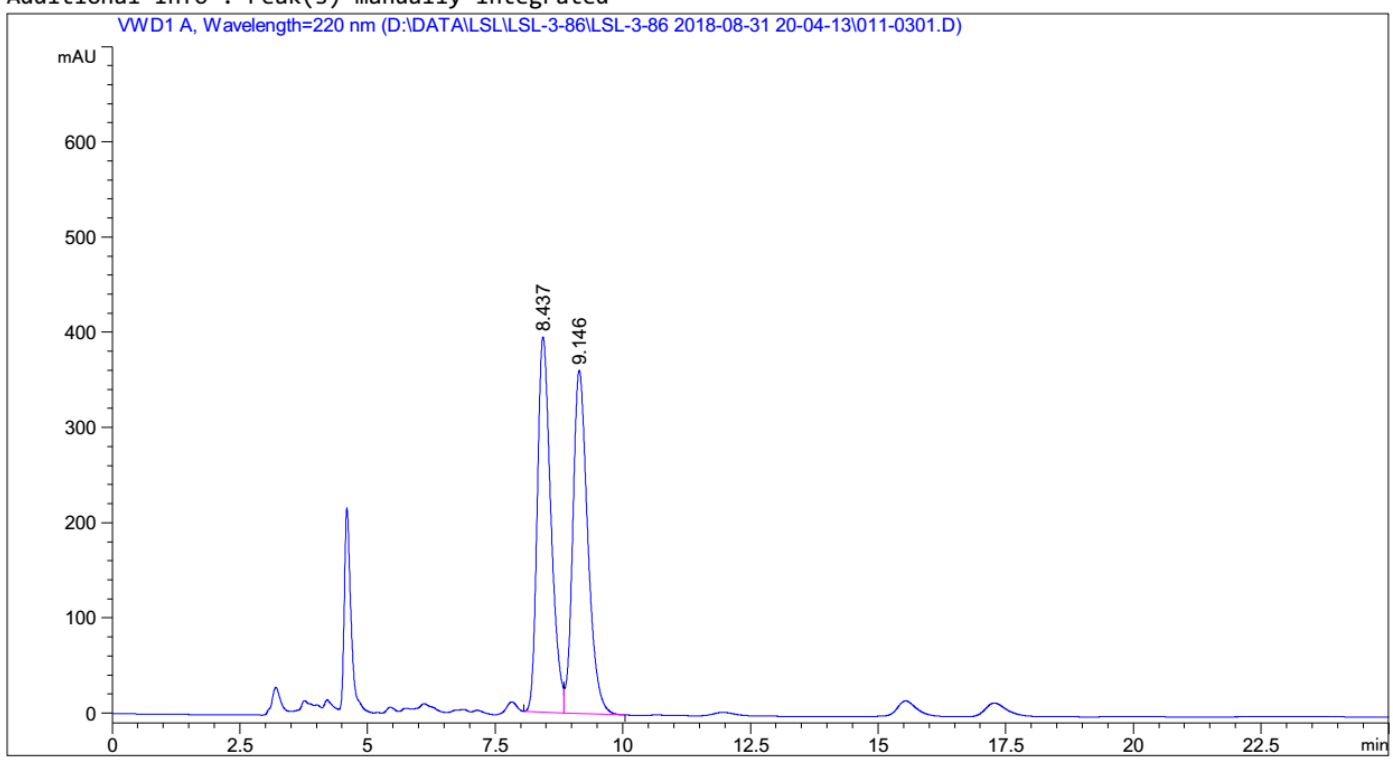

Area Percent Report

\begin{tabular}{|c|c|c|}
\hline Sorted By & : & Signal \\
\hline Multiplier & : & 1.0000 \\
\hline Dilution & : & 1.0000 \\
\hline
\end{tabular}

Use Multiplier \& Dilution Factor with ISTDs

Signal 1: VWD1 A, Wavelength $=220 \mathrm{~nm}$

\begin{tabular}{cccccc}
$\begin{array}{c}\text { Peak RetTime Type } \\
\text { \# } \\
{[\mathrm{min}]}\end{array}$ & $\begin{array}{c}\text { Width } \\
{[\mathrm{min}]}\end{array}$ & $\begin{array}{c}\text { Area } \\
{[\mathrm{mAU} \text { s }]}\end{array}$ & $\begin{array}{c}\text { Height } \\
{[\mathrm{mAU}]}\end{array}$ & $\begin{array}{c}\text { Area } \\
\%\end{array}$ \\
\hline 1 & 8.437 BV & 0.2830 & 7300.12646 & 394.37567 & 49.9452 \\
2 & 9.146 VB & 0.3098 & 7316.14697 & 360.40045 & 50.0548 \\
& & & & & \\
Totals : & & $1.46163 e 4$ & 754.77612 &
\end{tabular}


Data File D: \DATA \LSL \LSL-3-86\LSL-3-86 2018-08-31 20-04-13\012-0401.D Sample Name: LSL-3-86

\begin{tabular}{|c|c|c|}
\hline Acq. Operator & : & Seq. Line : 4 \\
\hline Acq. Instrument & : Instrument 1 & Location : Vial 12 \\
\hline Injection Date & : 8/31/2018 9:27:36 PM & 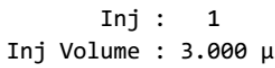 \\
\hline
\end{tabular}

Acq. Method : D: \DATA \LSL \LSL-3-86\LSL-3-86 2018-08-31 20-04-13\VWD-AS(1-6)-90-10-1ML-3UL -220NM-60MIN.M

Last changed : 8/31/2018 10:25:31 PM

(modified after loading)

Analysis Method: D: \METHOD\LWD \VWD-AD(1-2)-80-20-1ML-3UL-210NM-60MIN.M

Last changed : 9/25/2018 11:05:53 AM

(modified after loading)

Additional Info : Peak(s) manually integrated

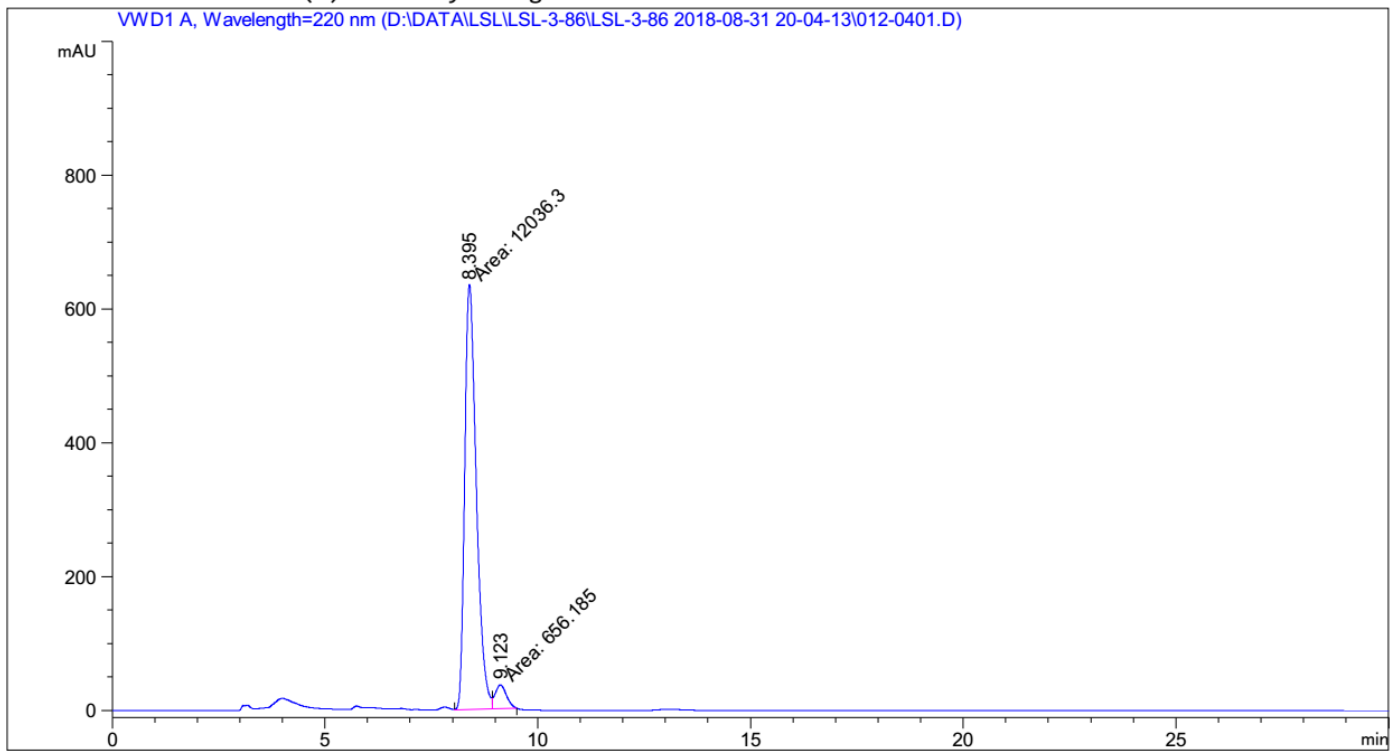

Area Percent Report

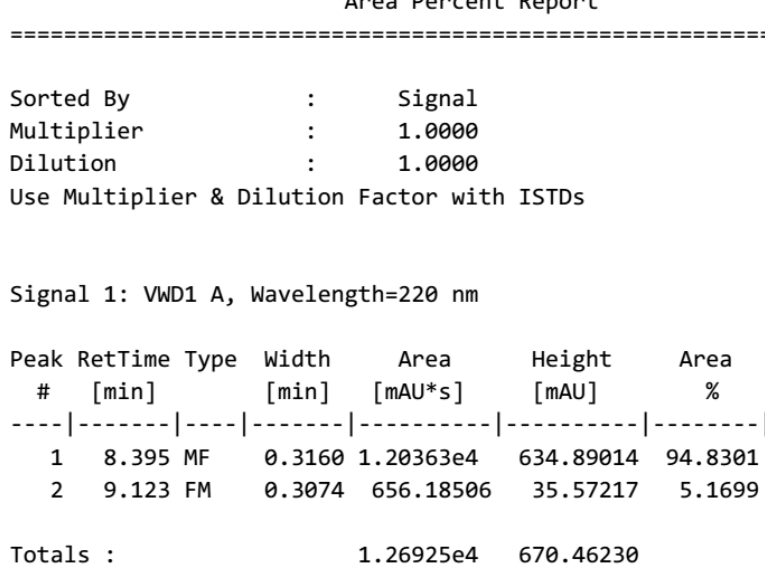


<smiles>COCC(CC=O)c1ccccc1</smiles>

Data File D: \DATA\LGY LGY-2-79\LGY-2-79-2 2018-09-18 17-22-04\022-0601.D Sample Name: LSL-3-104-2

\begin{tabular}{|c|c|c|}
\hline $\begin{array}{l}\text { Acq. Operator } \\
\text { Acq. Instrument }\end{array}$ & $\begin{array}{l}: \\
: \text { Instrument } 1\end{array}$ & $\begin{array}{r}\text { Seq. Line : } \quad 6 \\
\text { Location : Vial } 22\end{array}$ \\
\hline Injection Date & : 9/18/2018 7:37:20 PM & $\begin{array}{rlc}\text { Inj }: & 1 \\
\text { Inj Volume } & : & 5.000\end{array}$ \\
\hline
\end{tabular}

Acq. Method : D: \DATA \LGY \LGY-2-79\LGY-2-79-2 2018-09-18 17-22-04\VWD-AS(1-6)-97-3-1ML5UL-220NM-60MIN.M

Last changed : 5/29/2018 2:17:26 PM

Analysis Method : D: \METHOD\YCC \DAD-0J(1-6)-95-5-0.5ML-3UL-ALL-90MIN.M

Last changed : 9/29/2018 9:50:53 PM

(modified after loading)

Additional Info : Peak(s) manually integrated

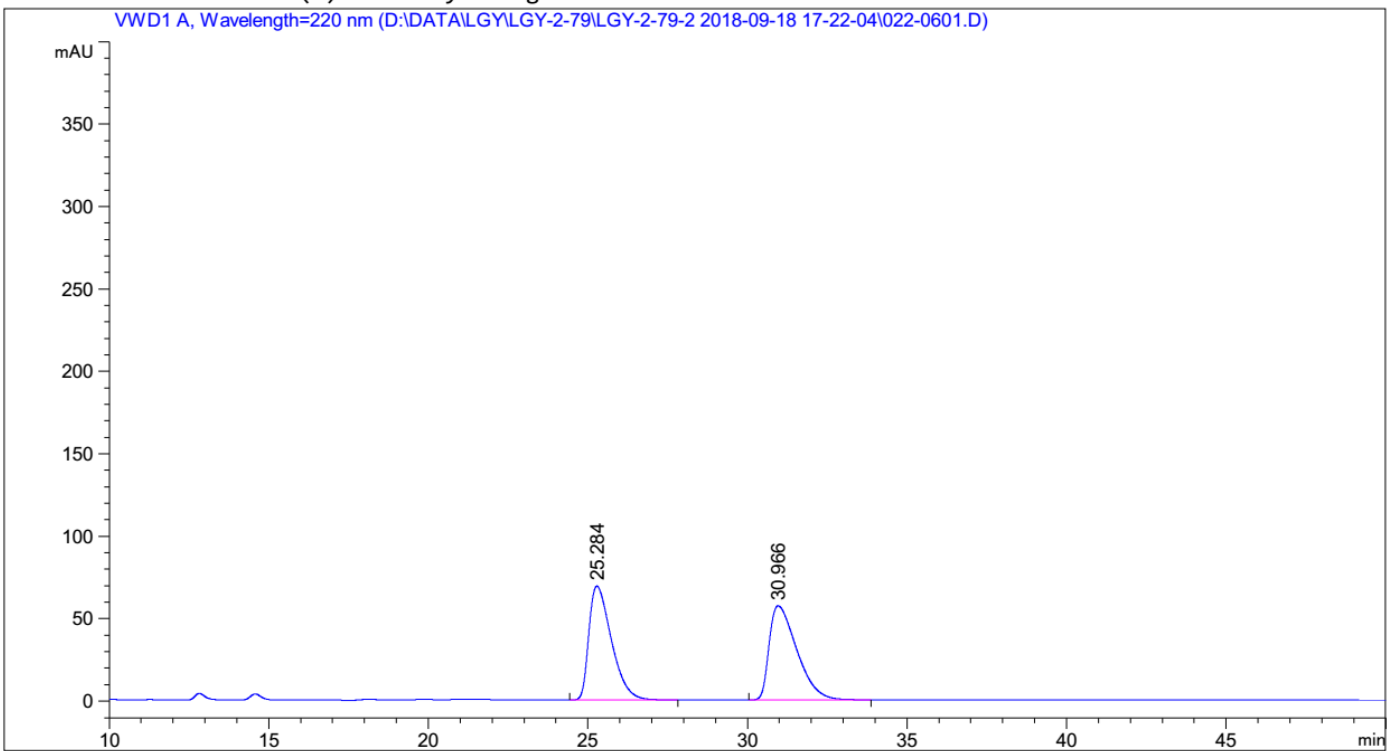

Area Percent Report

$\begin{array}{lll} & & \\ & & \\ \text { Sorted By } & : & \text { Signal } \\ \text { Multiplier } & : & 1.0000 \\ \text { Dilution } & : & 1.0000\end{array}$

Use Multiplier \& Dilution Factor with ISTDs

Signal 1: VWD1 A, Wavelength $=220 \mathrm{~nm}$

\begin{tabular}{|c|c|c|c|c|c|}
\hline $\begin{array}{c}\text { Peak } \\
\#\end{array}$ & $\begin{array}{l}\text { RetTime Type } \\
\text { [min] }\end{array}$ & $\begin{array}{l}\text { Width } \\
\text { [min] }\end{array}$ & $\begin{array}{c}\text { Area } \\
{\left[\mathrm{mAU}^{*} \mathrm{~s}\right]}\end{array}$ & $\begin{array}{l}\text { Height } \\
{[\mathrm{mAU}]}\end{array}$ & $\begin{array}{c}\text { Area } \\
\%\end{array}$ \\
\hline$=-$ & (1) & 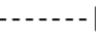 & |-.... & | & | \\
\hline 1 & 25.284 BB & 0.7592 & 3390.98193 & 69.01722 & 49.7044 \\
\hline 2 & 30.966 ВВ & 0.9298 & 3431.30908 & 57.04049 & 50.2956 \\
\hline Tota & : & & 6822.29102 & 126.05771 & \\
\hline
\end{tabular}


Data File D: \DATA \GU...QING \GYQA-ADDED \GYQA-180920-ME-COOET 2018-09-20 09-53-52\012-1901.D Sample Name: LSL-3-105-2

\begin{tabular}{|c|c|c|}
\hline Acq. Operator & : & Seq. Line : 19 \\
\hline Acq. Instrument & : Instrument 1 & Location : Vial 12 \\
\hline Injection Date & : 9/20/2018 9:07:53 PM & 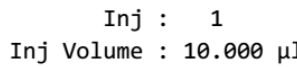 \\
\hline
\end{tabular}

Acq. Method : D: \DATA \GUAN YUQING \GYQA-ADDED \GYQA-180920-ME-COOET 2018-09-20 09-53-52\VWD -AS(1-6)-97-3-1ML-10UL-210NM-60MIN.M

Last changed : 9/20/2018 8:04:15 PM

Analysis Method : D: \METHOD \YCC \DAD-0J(1-6)-95-5-0.5ML-3UL-ALL-90MIN.M

Last changed : 9/29/2018 9:47:14 PM

(modified after loading)

Additional Info : Peak(s) manually integrated

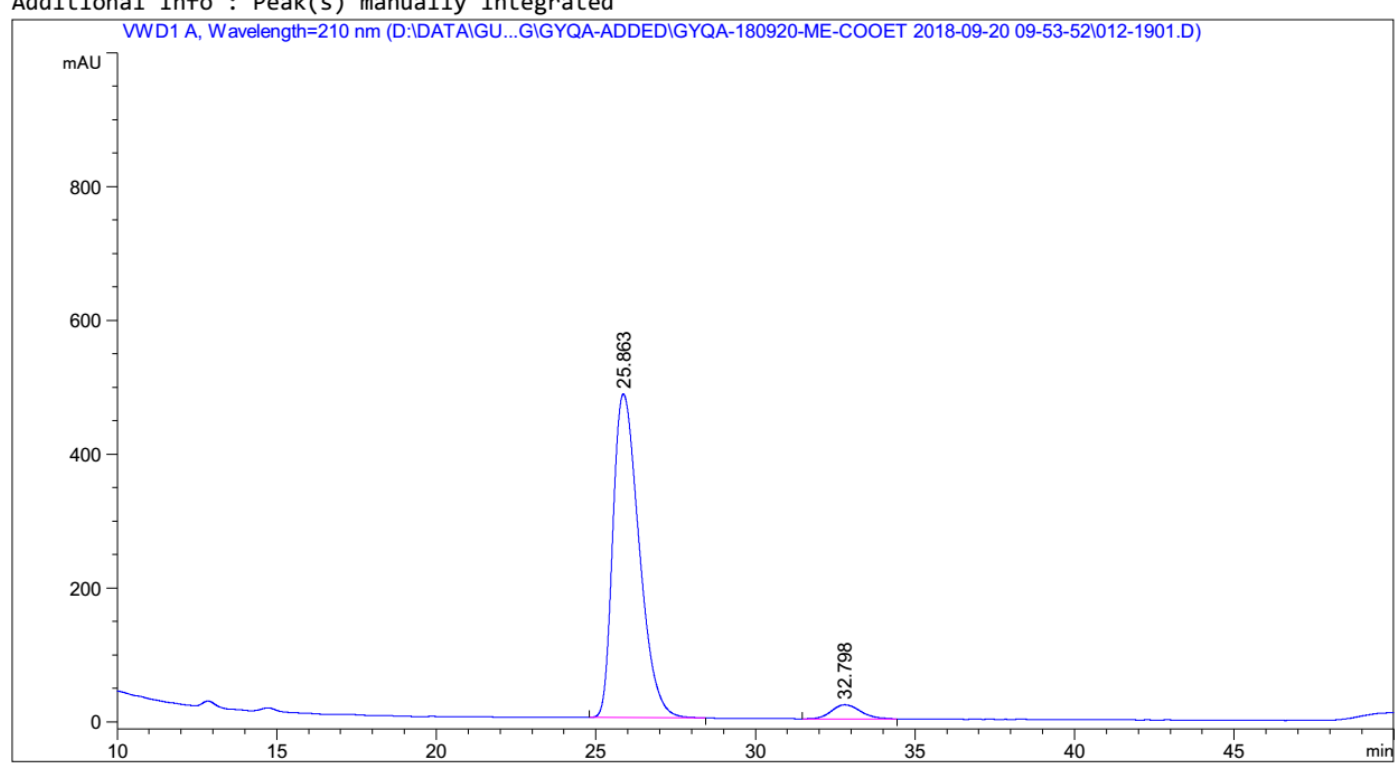

Area Percent Report

Sorted By : : Signal

Multiplier $\quad$ : 1.0000

Dilution : 1.0000

Use Multiplier \& Dilution Factor with ISTDs

Signal 1: VWD1 A, Wavelength $=210 \mathrm{~nm}$

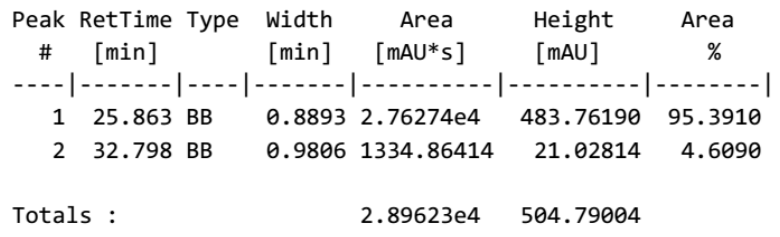

